\title{
Summary Report on the Industry Forum for Monitoring, Diagnostics, and Prognostics for Manufacturing Operations
}

Brian A. Weiss Michael Brundage

Yannick Tamm

Tommi Makila

Joan Pellegrino

This publication is available free of charge from: https://doi.org/10.6028/NIST.AMS.100-23 


\title{
Summary Report on the Industry Forum for Monitoring, Diagnostics, and Prognostics for Manufacturing Operations
}

\author{
Brian A. Weiss \\ Michael Brundage \\ Engineering Laboratory \\ National Institute of Standards and Technology \\ Yannick Tamm \\ Tommi Makila \\ Joan Pellegrino \\ Energetics
}

This publication is available free of charge from:

https://doi.org/10.6028/NIST.AMS.100-23

April 2019

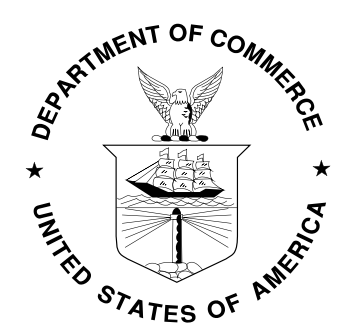

U.S. Department of Commerce

Wilbur L. Ross, Jr., Secretary

National Institute of Standards and Technology Walter Copan, NIST Director and Under Secretary of Commerce for Standards and Technology 
Certain commercial entities, equipment, or materials may be identified in this document in order to describe an experimental procedure or concept adequately. Such identification is not intended to imply recommendation or endorsement by the National Institute of Standards and Technology, nor is it intended to imply that the entities, materials, or equipment are necessarily the best available for the purpose.

The opinions, recommendations, findings, and conclusions in this publication do not necessarily reflect the views or policies of NIST or the United States Government.

National Institute of Standards and Technology Advanced Manufacturing Series 100-23

Natl. Inst. Stand. Technol. Adv. Man. Ser. 100-23, 332 pages (April 2019)

This publication is available free of charge from: https://doi.org/10.6028/NIST.AMS.100-23 


\title{
Disclaimer
}

This report is a collaborative effort between personnel from the National Institute of Standards and Technology (NIST) and Energetics. The views and opinions expressed herein do not necessarily state or reflect those of NIST or Energetics. Certain commercial entities, equipment, or materials may be identified in this document to illustrate a point or concept adequately. Such identification is not intended to imply recommendation or endorsement by the NIST or Energetics, nor is it intended to imply that the entities, materials, or equipment are necessarily the best available for the purpose.

\begin{abstract}
The National Institute of Standards and Technology (NIST) hosted the Industry Forum: Advanced Monitoring, Diagnostic, and Prognostic for Manufacturing Operations on May 811, 2018, on the NIST Gaithersburg (Maryland) campus. The purpose of the Industry Forum was to discuss the current trends, successes, challenges, and needs related to advanced monitoring, diagnostic, and prognostic technologies (collectively known as Prognostics and Health Management (PHM)) for enhancing maintenance and control strategies within manufacturing operations. The conclusion of the event featured an American Society of Mechanical Engineers (ASME) Standards meeting for the Advanced Monitoring, Diagnostics, and Prognostics for Manufacturing Operations Subcommittee ${ }^{1}$ to prioritize work activities for standards and guidelines. This report documents the four-day event, summarizing presentations and brainstorming sessions, in addition to detailing the key priority topics for standards and guidelines. The next steps in this effort are presented towards the end of this document, including planning for the next standards meeting (which has since occurred in October 2018 in Cincinnati, Ohio) and the 2019 Measurement and Evaluation for Prognostics and Health Management in Manufacturing Forum (ME4PHM) workshop.
\end{abstract}

\section{Key words}

Diagnostics; Factory Operations Planning \& Control; Manufacturing; Monitoring; Process Improvement; Process Measurement \& Control; Prognostics; Standardization; Forum.

${ }^{1}$ This Subcommittee was officially approved by ASME's Board of Directors shortly after this specific meeting occurred. 


\section{Table of Contents}

1 Introduction .................................................................................................................................. 1

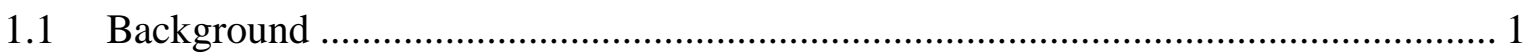

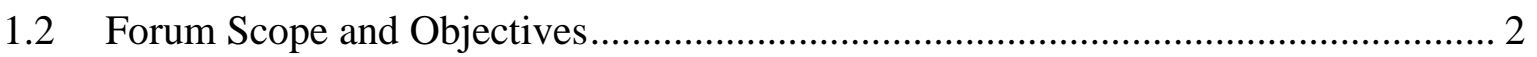

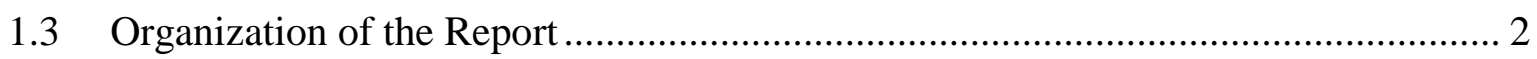

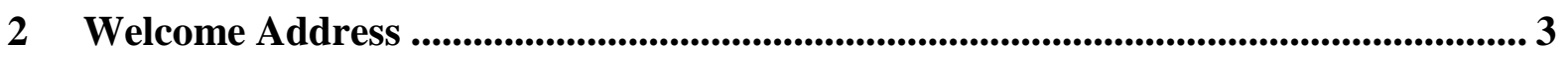

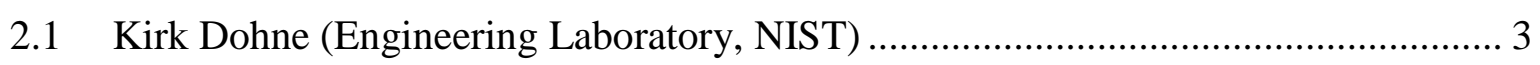

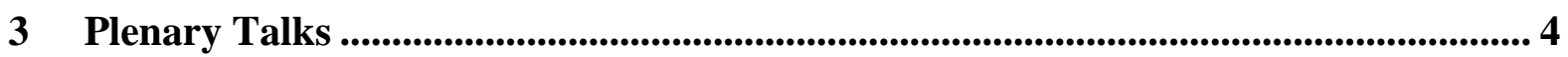

3.1 Manufacturing USA - Michael Molnar (Advanced Manufacturing National Program

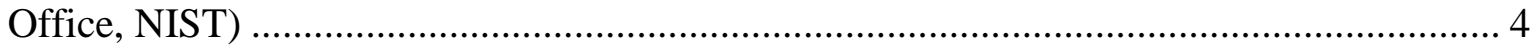

3.2 NIST Smart Manufacturing Research - Albert Wavering (Intelligent Systems

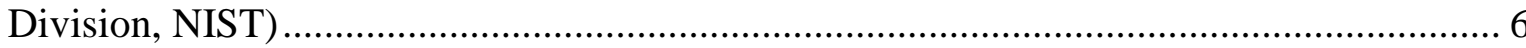

3.3 Process Monitoring and Diagnosis - Jamie Camelio (Commonwealth Center for Advanced Manufacturing).......................................................................................... 7

4 Costs and Benefits of Advanced Maintenance ................................................................ 8

4.1 Doug Thomas (Applied Economics Office, NIST) …………………………….... 8

5 Manufacturing State of the Art .........................................................................9

$5.1 \quad$ Large Manufacturing Needs and Case Studies.................................................... 9

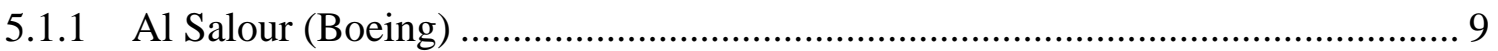

5.1.2 Luis Hernandez (Global Strategic Solutions) .................................................... 10

5.1.3 Michael Armacost (Applied Materials) ............................................................... 11

5.2 Small to Medium Manufacturing Needs and Case Studies ........................................ 13

5.2.1 Scott Sipe (Mantec) .................................................................................... 13

5.2.2 Tom Zbell (Genedge) .................................................................................... 14

5.2.3 Thorsten Wuest (West Virginia University) ....................................................... 14

5.2.4 Mark Walker (D2K) ............................................................................... 15

$6 \quad$ Design and Implementation of PHM .............................................................................. 17

6.1 Planning and Assessment to Promote PHM Technologies ....................................... 17

6.1.1 Karl Reichard (Penn State University Applied Research Lab) .......................... 17

6.1.2 Ananth Seshan (MESA) ............................................................................ 17

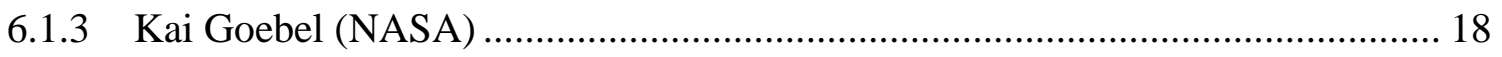

6.1.4 Miguel Saez (University of Michigan)......................................................... 18

6.1.5 Jorge Arinez (General Motors (GM)) ……………...................................... 18

6.2 Emerging Sensing Technologies to Enable PHM …………………………….... 20

6.2.1 Radu Pavel (TechSolve) ............................................................................ 20 
6.2.2 Brittany Newell (Purdue University) ............................................................... 21

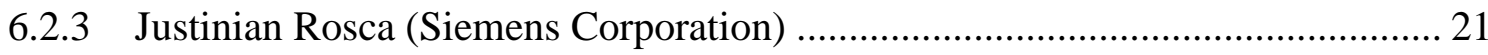

6.2.4 Gregory Vogl (NIST - Intelligent Systems Division) ....................................... 22

6.2.5 Ed Spence (Machine Instrumentation) ……………........................................ 22

6.3 Monitoring and Analysis Technologies for PHM ……....................................... 23

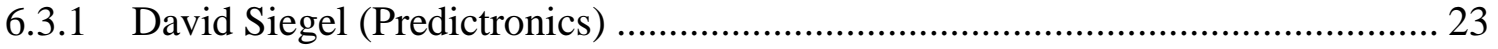

6.3.2 Nancy Diaz-Elsayed (University of South Florida) ………………………........ 24

6.3.3 Sanket Amberkar (Falkonry) ...................................................................... 25

6.3.4 Robert Gao (Case Western Reserve University) ................................................ 25

6.3.5 ChaBum Lee (Tennessee Tech University)....................................................... 25

6.4 Communication and Information Flow to Support PHM....................................... 28

6.4.1 Will Sobel (Vimana) ………….................................................................. 28

6.4.2 Moneer Helu (NIST Systems Integration Division)............................................ 29

6.4.3 Joel Neidig (ITAMCO) ................................................................................. 29

6.4.4 Rob Andes (The Design Knowledge Company) ……........................................... 30

$7 \quad$ Additional NIST Measurement Science Research Efforts............................................. 32

7.1 Michael Brundage (Systems Integration Division, NIST) ...................................... 32

7.2 Brian A. Weiss (Intelligent Systems Division, NIST) ………………………….... 32

8 Standards and Best Practices .................................................................................... 33

$8.1 \quad$ Emerging Research Efforts ………….............................................................. 33

8.1.1 Junmin Lee (Seoul National University) ............................................................ 33

8.2 Panel on Standardization and Best Practices......................................................... 33

8.2.1 Andrew Hess (Hess PHM Group) ………………............................................ 33

8.2.2 Ravi Rajamani (drR2) ............................................................................ 34

8.2.3 Tom Fiske (Yokogawa) .............................................................................. 34

8.2.4 Tom Hedberg (NIST - Systems Integration Division) ……………………........ 35

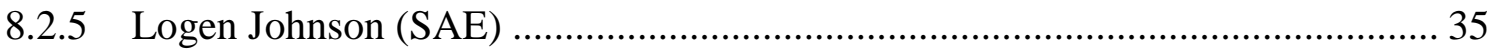

8.2.6 Donnie Alonzo (ASME) ............................................................................ 36

9 International Perspectives on PHM.................................................................................. 38

9.1 Byeng Youn (Seoul National University) ............................................................ 38

9.2 Minchul Lee (Pohang University of Science and Technology) ................................. 39

9.3 Hyunseok Oh (Gwangju Institute of Science and Technology) ................................ 40

9.4 Chan Hee Park (Seoul National University) …………………………………..... 40

10 Visualization Tools for PHM ............................................................................................... 41 
10.1 Jeremy Marvel (Intelligent Systems Division, NIST) ........................................... 41

10.2 Sinan Bank (Siemens Corporation) ........................................................................ 41

11 Planning for the Future - Building and Leveraging Artificial Intelligence.............. 43

11.1 Michael Garris (Information Technology Laboratory, NIST) ................................. 43

11.2 Jay Lee (University of Cincinnati, Center for Intelligent Maintenance Systems) .... 45

12 ASME Standards Meeting .................................................................................................. 47

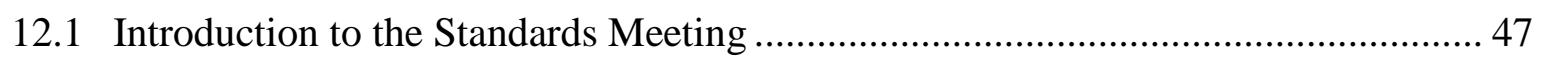

12.2 ASME Efforts in Monitoring, Diagnostics, and Prognostics - Donnie Alonzo and

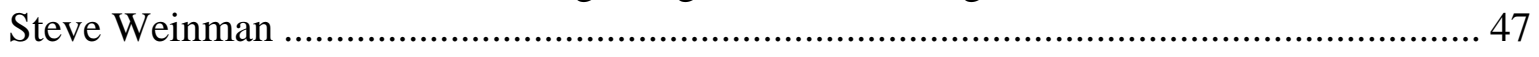

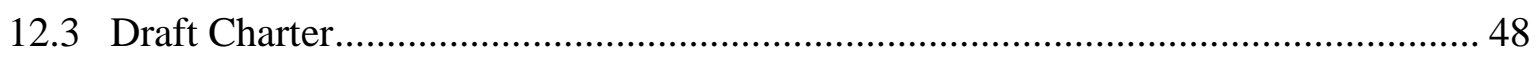

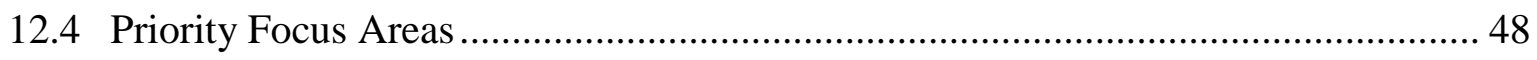

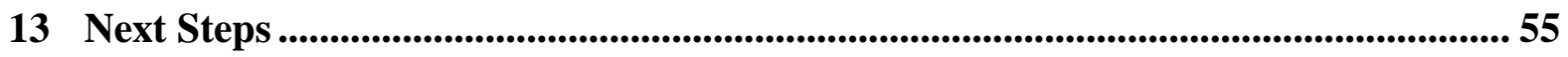

13.1 ASME Face-to-Face Subcommittee Meetings ……………………………........ 55

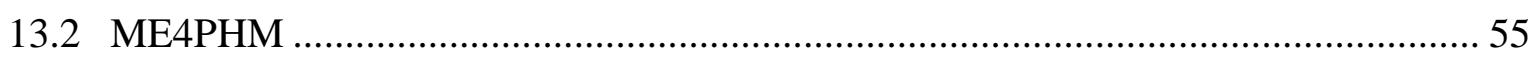

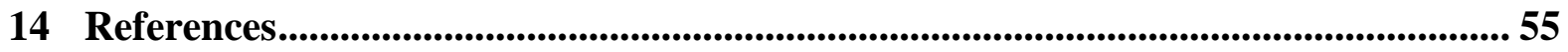

Appendix A - Acronyms ............................................................................................................ 57

Appendix B - Industry Forum Conference Program......................................................... 61

Appendix C - Participant List .............................................................................................. 62

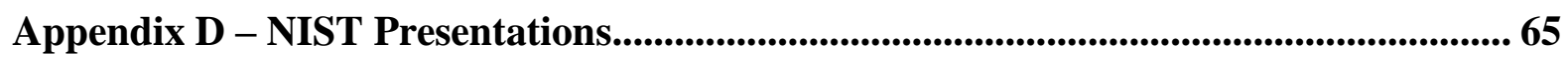

\section{List of Tables}

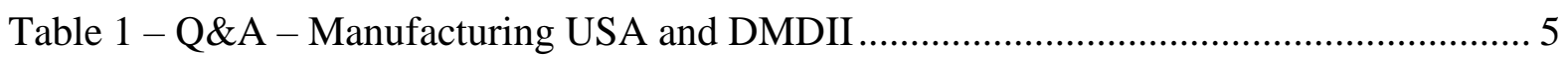

Table 2 - Q\&A for Smart Manufacturing Programs ………................................................ 7

Table 3 - Q\&A - Process Monitoring and Diagnosis .............................................................. 8

Table 4 - Q\&A - Large Manufacturing Needs and Case Studies Panel ................................. 11

Table 5 - Q\&A - Small to Medium Manufacturing Needs/Case Studies Panel .................... 15

Table 6-Q\&A - Planning and Assessment to Promote Monitoring, Diagnostics and

Prognostic Technologies ..................................................................................................... 19

Table 7 - Q \&A - Emerging Sensing Technologies to Enable Monitoring, Diagnostics, and

Prognostics.... 23

Table 8 - Q\&A - Panel on Monitoring and Analysis Technologies for Prognostics and

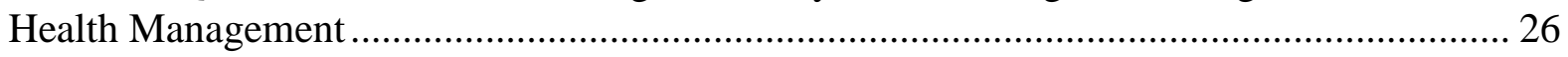

Table 9 - Q\&A - Monitoring and Analysis Technologies for Prognostics and Health

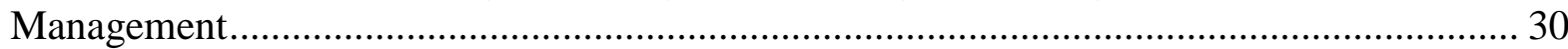

Table 10 - Q\&A - Standards and Best Practices .................................................................... 37

Table 11-Q\&A - PHM Frontiers in Korean Manufacturing - Success Episodes and Issues

Q A - Visualization Tools for PHM

Table 12 - Q\&A - Visualization Tools for PHM ................................................................ 42

Table 13 - Q\&A - Demystifying today's Artificial Intelligence .............................................. 44 
Table 14 - Q\&A - A System Perspective in Machine Learning for Smart Manufacturing and

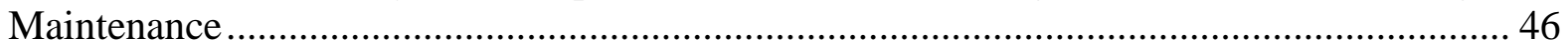

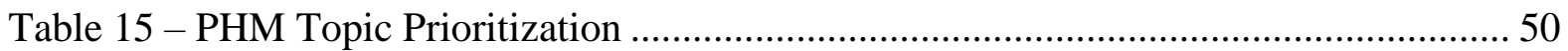

\section{List of Figures}

Figure 1: NIST Laboratories and Facilities ................................................................ 3

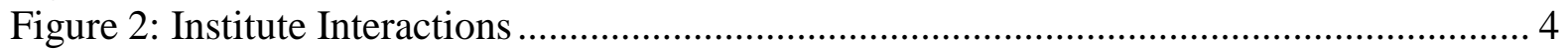

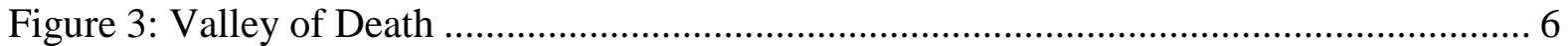

Figure 4: Description of IVHM Capability Level....................................................... 10

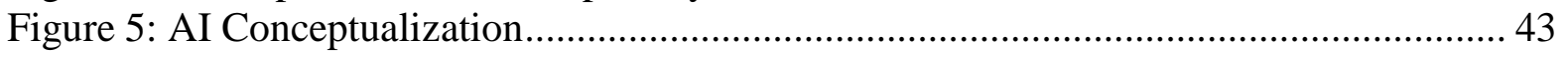

Figure 6: Priority Guideline Action Plan for Topic 2 - Guideline to Determine What Health

Data to Capture and Collection Strategies to Employ ...................................................... 51

Figure 7: Priority Guideline Action Plan for Topic 5 - Guideline to Determine Where and

When PHM Should be Added/Integrated ..................................................................... 53

Figure 8: Priority Guideline Action Plan for Topic 7 - Guideline to Determine Where PHM

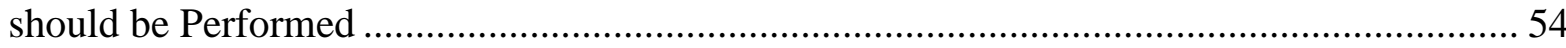




\section{Introduction}

\subsection{Background}

Many manufacturers lack the knowledge to effectively design and implement advanced monitoring, diagnostic, and prognostic (collectively known as Prognostics and Health Management (PHM)) technologies across a wide array of factory floor-level processes and equipment to optimize their maintenance and control strategies [1-3]. The manufacturing community has been evolving as new technologies emerge, existing technologies mature, and advanced technologies become accessible due to lower costs and ease of integration. New fault and failure modes emerge as technologies integrate into manufacturing operations for the first time and existing processes become reconfigured to support new products. New sensors are gathering more information than ever before; processed analytics offer greater intelligence and awareness. Effectively using PHM will minimize unplanned downtime and optimize planned downtime leading to greater asset availability. Likewise, PHM can help maintain process quality and productivity targets, and minimize waste (e.g., excess raw material) all towards reducing cost, and increasing profit and efficiency.

Manufacturers take different approaches, especially between large and small to mediumsized enterprises (SMEs), to deal with these faults and failures [4-6]. Most manufacturers aim to avoid reactive maintenance - fix equipment after it breaks and interrupts production - as this strategy can present substantial risk and cost. Nearly every manufacturer employs some form of preventive maintenance - perform a predefined maintenance routine at prescribed intervals (e.g., number of hours) - as a means to keep their systems and processes operating within specification. While this strategy typically is more cost effective than reactive maintenance and usually promotes a safer operating environment, it can sometimes lead to unnecessary cost and downtime if maintenance occurs too frequently. Likewise, it still does not eliminate the prospect of reactive maintenance. Some in the manufacturing community are increasing their predictive maintenance capability planning maintenance activities based upon analyzing specific sensor data that inform upon system and process performance and health. This 'right-on-time' strategy moves closer to optimizing maintenance activities to minimize both downtime and maintenance costs. Proactive maintenance, intelligent maintenance, and autonomous maintenance are emerging strategies that present tremendous potential for further minimizing equipment and process downtime. All of these strategies have benefits and challenges, so it may be difficult for a manufacturer to determine where to begin. All manufacturers are different so no single solution will solve every maintenance challenge.

PHM seeks to advance maintenance strategies such that manufacturers can monitor those metrics they deem critical to operations and process/equipment health, and effectively determine what maintenance needs to be performed, and when, to minimize asset downtime.

To that effect, the development of standards and guidelines that describe and promote advanced monitoring, diagnostic, and prognostic technologies, along with ways of verifying and validating their performance, to enhance maintenance and control strategies 
within manufacturing operations at the factory floor, would be beneficial to the manufacturing industry [7-9].

\subsection{Forum Scope and Objectives}

The National Institute of Standards and Technology (NIST) hosted the "Industry Forum: Monitoring, Diagnostics, and Prognostics for Manufacturing Operations" (noted as "Industry Forum" throughout the remainder of this report) from May 8-11, 2018 at the NIST Campus in Gaithersburg, Maryland. Appendix B - Industry Forum Conference Program - provides a detailed agenda for the four-day event.

The goal of the Industry Forum is to bring industry, government, and academia together to discuss the current trends, successes, challenges, and needs with respect to advanced monitoring, diagnostic, and prognostic technologies to enhance maintenance and control strategies within manufacturing operations. Participants discussed current and emerging capabilities and challenges with respect to designing, deploying, verifying, and validating monitoring, diagnostic, and prognostic technologies for manufacturing operations including those involving interconnected, Internet of Things (IoT) technologies.

The Industry Forum was an open event, attended by 72 external stakeholders and 21 NIST representatives. The target audience included members of the manufacturing, monitoring, diagnostic, and prognostic communities, including technology developers, technology integrators, end-users (including both large manufacturers and SMEs), researchers (from academia and other organizations), and government entities.

The first three days of the Industry Forum were composed of presentations and panels featuring:

1. Manufacturers' successes and challenges with respect to their maintenance strategies

2. Technology integrators' and technology developers' emerging hardware and software capabilities to enhance equipment and process intelligence

3. Verification and validation techniques to expand the boundaries of what parameters can be reasonably measured and assessed to enhance end-user confidence in PHM capabilities

The fourth and final day of the Industry Forum was devoted to building up an American Society of Mechanical Engineers (ASME)-led standards community focused on advanced monitoring, diagnostics, and prognostics for manufacturing operations. Donnie Alonzo and Steve Weinman from ASME, along with Brian A. Weiss and Michael Brundage from NIST guided the participants in examining specific priority areas and detailing the next steps to generate and deliver guidelines to industry that enhance a manufacturer's ability to design, deploy, verify, and validate their maintenance-related capabilities.

\subsection{Organization of the Report}

This report summarizes the presentations of plenary speakers, panelists, and others. Appendix D - NIST Presentations includes presentations given by NIST speakers. This 
report also summarizes the panel question/answer (Q/A) sessions following the plenary and panelist presentations. Presentation and discussion summaries are organized by topic area rather than chronological agenda order.

\section{Welcome Address}

\subsection{Kirk Dohne (Engineering Laboratory, NIST)}

“Overview of the NIST Engineering Laboratory" (May 8)

Mr. Dohne, Associate Director of the Engineering Laboratory at NIST, opened the forum by providing a high-level overview of the NIST Engineering Laboratory ${ }^{2}$. The Engineering Laboratory is one of several laboratories and facilities that house NIST's research activities. The mission of the Engineering Laboratory is to promote U.S. innovation and industrial competitiveness by advancing measurement science, standards, and technology for engineered systems in ways that enhance economic security and improve our quality of life. NIST and the Engineering Laboratory play an essential Federal Government role by developing new measurement science, standards, and technology throughout manufacturing. NIST's reputation and neutrality enable the convening of diverse stakeholders to address complex technical issues.

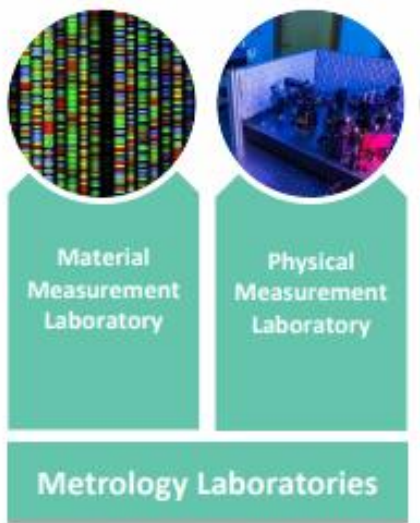

Driving innovation through Measurement Science and Standards
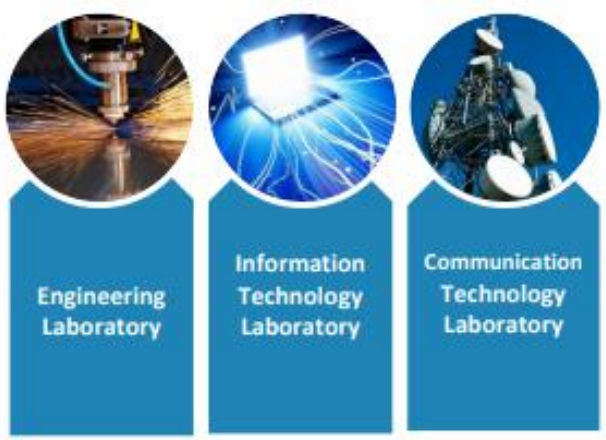

Technology Laboratories

Accelerating the adoption and deployment of advanced technology solutions

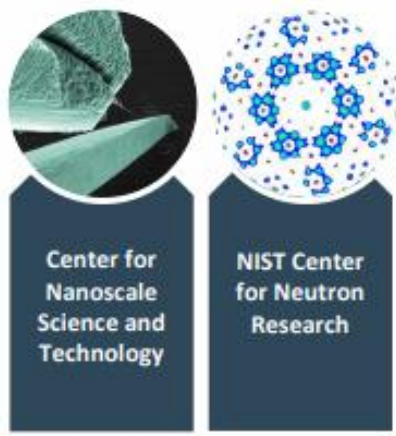

National User Facilities

Providing world class, unique, and cutting-edge research facilities

Figure 1: NIST Laboratories and Facilities

Measurements are critical to both commerce, and innovation. Measurement enables one to design, improve, and compare technology, while providing the foundation for innovation in every industry and economic sector. NIST provides support to industry and government to develop voluntary standards by leveraging expert participation including NIST technical

${ }^{2}$ https://www.nist.gov/el 
staff and international standards bodies such as the American Society of Mechanical Engineers $\left(\mathrm{ASME}^{3}\right)$.

NIST is undertaking substantial work in smart manufacturing ${ }^{4}$ related to operations, planning and controls, systems design and analysis, robotics systems, and additive manufacturing. In general, NIST helps U.S. manufacturers to invent, innovate, and create by developing precision test methods, tools, and data, and facilitating public-privateacademic partnerships.

Mr. Dohne's slide deck is included in Appendix D - NIST Presentations.

\section{Plenary Talks}

\subsection{Manufacturing USA - Michael Molnar (Advanced Manufacturing National Program Office, NIST)}

“Manufacturing USA and DMDII - Program Update and Activities in PHM" (May 8)

Mr. Molnar provided an overview of how the Advanced Manufacturing National Program Office (AMNPO) supported work at the Manufacturing USA institutes and shared results of PHM-related projects. The AMNPO, a federal interagency office headquartered at NIST, has the power to convene multiple organizations to achieve common objectives. The office oversees Manufacturing USA - a network of regional institutes, each with a specialized technology focus. As shown in Figure 2, this network connects people, ideas and technology to solve relevant challenges for advanced manufacturing to enhance the competitiveness of U.S. manufacturers. Each institute develops and owns a unique technology roadmap, and hosts workshops to help guide and prioritize research. Roadmaps also inform the development of a balanced portfolio of institute projects.

Each institute has a clear mission, working to generate value for industry participants and create an effective collaboration space for applied pre-competitive applied research and development (R\&D). Institute work is guided by industry-led consortia and focuses on priorities and challenges that greatly benefit from substantial

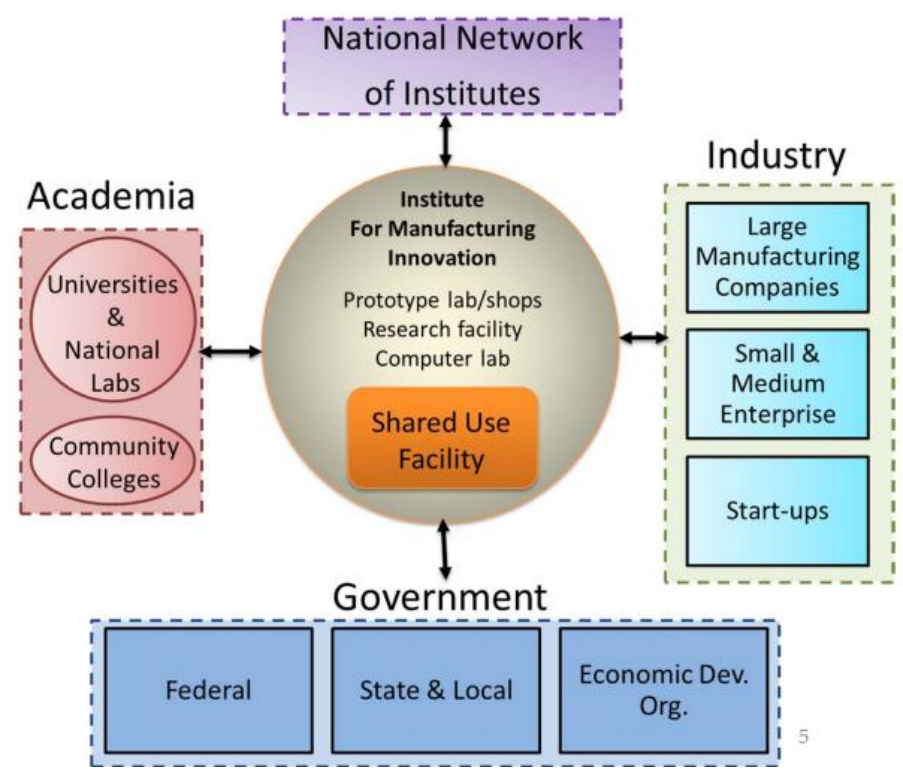

Figure 2: Institute Interactions

${ }^{3}$ https://www.asme.org/

${ }^{4}$ https://www.nist.gov/el/goals-programs/smart-manufacturing 
collaboration. Federal start-up funding for each institute must catalyze at least $100 \%$ coinvestment from non-federal partners. Along with technology advancement, institutes address education and workforce skills gaps related to their respective emerging technologies. The institutes aim to "speed-up and de-risk" technologies, to bridge the gap from discovery to production. The institutes delivered real value in 2017; institute membership of industry, academia and government increased by $50 \%$, and included 273 advanced R\&D projects with an overall 60/40 split achieved in non-federal/federal funding.

The Digital Manufacturing and Design Innovation Institute (DMDII) is one example of a successful institute. This institute sponsors many PHM activities, including tools to help manufacturers integrate PHM in their operations. In one project, a reconfigurable retrofit kit for legacy equipment is under development that provides significant flexibility and state-of-the-art network security. The prospective $\$ 300$ kit would be open source and cyber-secure. A framework for cloud-based online machine and process monitoring, diagnosis, and prognosis is also under development. Another example is improving manufacturing operations by combining traditional manufacturing execution system (MES) technology metrics like overall equipment effectiveness (OEE) with in-depth predictive maintenance analytics.

Mr. Molnar's slide deck is included in Appendix D - NIST Presentations.

A brief question and answer (Q\&A) session is summarized in Table 1 - Q\&A Manufacturing USA and DMDII

Table 1 - Q\&A - Manufacturing USA and DMDII

\begin{tabular}{|l|l|}
\hline Question: & $\begin{array}{l}\text { Regarding the graph showing the gap (a.k.a., 'valley of death' - shown in } \\
\text { Figure 3) between innovation in the laboratory and commercialization, are } \\
\text { we narrowing this gap to achieve greater and/or faster commercialization? }\end{array}$ \\
\hline Answer: & $\begin{array}{l}\text { This chart shows how other countries have used government funding to bridge } \\
\text { this gap (i.e., valley of death). In the U.S., we are focusing on letting industry } \\
\text { do more of the R\&D and playing a role in bringing industry together. We want } \\
\text { to create a collaborative space where industry can do more precompetitive } \\
\text { R\&D. }\end{array}$ \\
\hline Question: & $\begin{array}{l}\text { Is it correct that most of the projects reviewed do not involve standards } \\
\text { development? }\end{array}$ \\
\hline Answer: & $\begin{array}{l}\text { They are involved with standards settings. Most projects actually have a } \\
\text { standards theme. }\end{array}$ \\
\hline
\end{tabular}




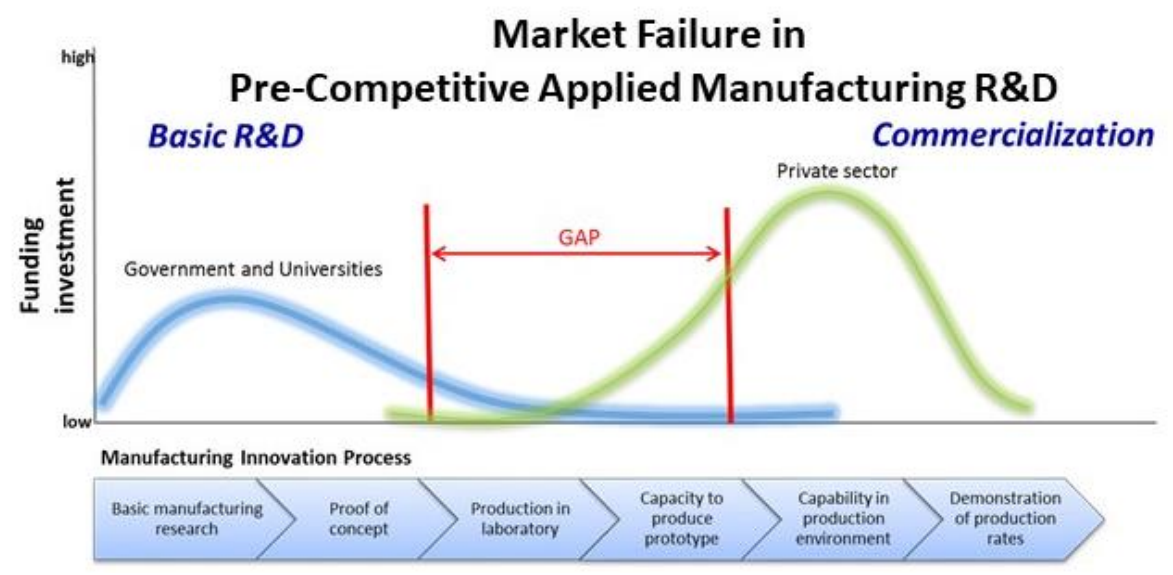

Figure 3: Valley of Death

\subsection{NIST Smart Manufacturing Research - Albert Wavering (Intelligent Systems Division, NIST)}

"NIST Smart Manufacturing Programs: Driving Innovation and Reducing Risks of Adoption of New Technologies" (May 9)

Mr. Wavering presented highlights of NIST's smart manufacturing programs, which help drive innovation and reduce the risk of adoption of emerging and disruptive manufacturing technologies. These programs contribute to standards (including interoperability standards) that provide a common language along with test methods that technology developers, integrators, and users can deploy to assess, verify, and validate technical capabilities and performance. A critical aspect of this is that the standards and test methods aim to be device-agnostic; they should be reasonably applicable to all technical solutions within a technological category.

Industry input, and industry user needs in particular, motivates NIST research; it is the first step to developing measurement science (e.g., performance metrics, test methods, reference datasets) and industry-driven standards. After industry input is captured, advanced/emerging technologies are identified that would benefit from measurement science to verify and validate (V\&V) performance. NIST researchers then develop the technical basis to enable $\mathrm{V} \& \mathrm{~V}$. This technical basis is then included in standards, where appropriate, for industry to leverage.

NIST's smart manufacturing research efforts are comprised of four programs within the Intelligent Systems Division ${ }^{5}$ and Systems Integration Division. ${ }^{6}$ These include Measurement Science for Additive Manufacturing, Robotic Systems for Smart Manufacturing, Smart Manufacturing Systems Design and Analysis, and Smart Manufacturing Operations Planning and Control. NIST contributes to standards development in all four areas.

\footnotetext{
${ }^{5}$ https://www.nist.gov/el/intelligent-systems-division-73500

${ }^{6}$ https://www.nist.gov/el/systems-integration-division-73400
} 
Examples of ongoing work at NIST relating to PHM include Manufacturing Process and Equipment Monitoring, Health and Control Management for Robot Systems, and Machine Tool Linear Axes Diagnostics and Prognostics. These efforts focus on developing use cases, test methods, performance metrics, reference data sets, and tools to enable verification and validation of monitoring, diagnostic, and prognostic technologies across the manufacturing factory floor. Additive manufacturing (AM) is an emerging area for PHM. Given that AM is still evolving as a technology, applying advanced PHM techniques may be somewhat premature. However, equipment manufacturers are currently starting to build in some monitoring capabilities that may be useful for PHM. AM machines contain many critical subsystems with potential failure modes, and fully understanding these is in a nascent stage.

The talk ended with a question posed to the audience: What measurement science and standards will drive innovation and reduce risks of adoption of emerging/disruptive PHM technologies? ASME Standards discussions (final day) are expected to shed some light on the answers.

Mr. Wavering's slide deck is included in Appendix D - NIST Presentations.

A brief question and answer (Q\&A) session is summarized in Table 2 below.

Table 2 - Q\&A for Smart Manufacturing Programs

\begin{tabular}{|l|l|}
\hline Question: & $\begin{array}{l}\text { Participants challenged the notion that it is too early to think about PHM for } \\
\text { additive manufacturing. Understanding redundancies, sensors, etc. and } \\
\text { integrating PHM could give AM a competitive advantage. }\end{array}$ \\
\hline Answer: & $\begin{array}{l}\text { AM equipment suppliers should not be discouraged from including PHM as they } \\
\text { build. It would be helpful to understand the characteristics of each layer and what } \\
\text { happens throughout the building process, using imaging technology; process } \\
\text { subsystem monitoring would also be useful for maintenance. }\end{array}$ \\
\hline
\end{tabular}

\subsection{Process Monitoring and Diagnosis - Jamie Camelio (Commonwealth Center for Advanced Manufacturing)}

"CCAM Activities in Process Monitoring and Diagnostics" (May 10)

Dr. Camelio summarized the efforts of the Commonwealth Center for Advanced Manufacturing $\left(\mathrm{CCAM}^{7}\right)$, which performs manufacturing $\mathrm{R} \& \mathrm{D}$ at all technology readiness levels (TRLs), from basic research to demonstration and launch. ${ }^{8}$ Another goal of CCAM is to help develop the workforce for advanced manufacturing. CCAM waives its intellectual property rights, helping to create a more fluid process for collaboration. Their primary focus involves developing capabilities to support digital and intelligent factories. While many manufacturers would like adaptive systems immediately, it takes an iterative process to reach this goal. In support of their intelligent factory efforts, CCAM works on

\footnotetext{
${ }^{7}$ http://www.ccam-va.com/

${ }^{8}$ https://en.wikipedia.org/wiki/Technology readiness level
} 
acoustic emission and vibration sensors, measurement technologies such as laser and thermal imaging processing, and technology validation.

CCAM's work in process monitoring includes a complex project to connect 16 machines to experiment with dynamic scheduling and a unique dashboard. A barrier to predicting faults and failures is the lack of commensurate data. Prognosis requires re-creation of failure modes. Many companies that CCAM works with want a comprehensive PHM solution (e.g., methods to effectively monitor factory health and guide maintenance practices), so CCAM compiles a complete data package that aggregates all required sensor measurements and data requirements. To do this efficiently, industry requires effective data compression techniques, another area of focus for CCAM. Industry is also increasingly interested in in-situ measurements (sensing and measurements on the machine). Selecting which data to pass through is a challenge, as overwhelming the system with data is counterproductive. Table 3 below summarizes the brief Q\&A session related to this presentation.

Table 3 - Q\&A - Process Monitoring and Diagnosis

\begin{tabular}{|l|l|}
\hline Question: & $\begin{array}{l}\text { Regarding additive manufacturing monitoring, what fault modes } \\
\text { and features are you interested in? }\end{array}$ \\
\hline Answer: & $\begin{array}{l}\text { We are interested in predicting voids and fractures. We can "hear" the } \\
\text { cracks with acoustic monitoring. CCAM excels in this area. }\end{array}$ \\
\hline Question: & Are you working on prognostic R\&D with universities? \\
\hline Answer: & $\begin{array}{l}\text { Internal staff at CCAM conduct our prognostics work. We employ many } \\
\text { Ph.D.'s and recent graduates, and experts that formerly worked in } \\
\text { industry, and publish work in this area. }\end{array}$ \\
\hline Question: & What is the size limit for manufacturing parts at CCAM? \\
\hline Answer: & Approximately one foot by one foot. \\
\hline
\end{tabular}

\section{Costs and Benefits of Advanced Maintenance}

\subsection{Doug Thomas (Applied Economics Office, NIST)}

“The Costs and Benefits of Advanced Maintenance in Manufacturing” (May 8)

Mr. Thomas presented on how predictive maintenance can reduce operating costs. Research and data are required to estimate the cost for reactive, preventative, and predictive maintenance; this will be company-dependent. Current maintenance cost data has limitations and sometimes lacks granularity as it includes buildings with manufacturing equipment, rather than differentiating the maintenance costs between the two. The literature also varies in terms of metrics that are used to summarize maintenance costs, countries that have captured manufacturing maintenance data and at what level, and terminology that is used when talking about the various maintenance strategies (e.g., 
predictive maintenance vs. condition-based maintenance). An industry survey is needed to provide the data for this research. A minimal sample size of 14 to 77 companies from industry is required to make statistically significant estimates of maintenance costs (depending upon the desire confidence).

Mr. Thomas' slide deck is included in Appendix D - NIST Presentations.

\section{Manufacturing State of the Art}

\subsection{Large Manufacturing Needs and Case Studies}

\subsubsection{Al Salour (Boeing)}

"NIST Industry Forum: Monitoring, Diagnostics, and Prognostics for Large Manufacturing Operations" (May 8)

Dr. Salour, a Technical Fellow at Boeing Research and Technology, presented background information about The Boeing Company, a major U.S. commercial, defense and space aviation manufacturer employing over 160,000 people around the world. This included introduction of the equipment data flow architecture at Boeing, or the "Boeing Model." Machine performance and health monitoring is a closed loop activity in the Boeing Model. Machine learning intelligence supports health and maintenance activities. Boeing performs vibration and failure modes and effects analysis (FMEA) and fault detection as well as other techniques to monitor the health of components. There are many examples of FMEA error finding, machine learning for fault detection, and machine and infrastructure health monitoring at Boeing.

Boeing has many examples of sensor-based process monitoring systems. Boeing monitors environmental metrics in places such as freezers (e.g., material age) or clean rooms (e.g., particles in the air). Additionally, technology, such as Light Detection and Ranging (LiDAR) and cameras, monitor vehicle path and traffic patterns within their vehicle safety system. When introducing new technology, it is important to first determine data requirements including what data is needed, what data can be made available, and what can be done with the available data. This must be done prior to introducing new equipment into the organization.

Overall, Boeing relies on industry standards and support from both inside and outside of the company to develop and integrate prognostic and monitoring technologies. Boeing has many monitoring systems for unique purposes. All work jointly to ensure quality product and a safe environment. Boeing considers sensing capabilities as they acquire new equipment. The easiest time to implement PHM components is in the design and construction of a new facility. 


\subsubsection{Luis Hernandez (Global Strategic Solutions)}

"Condition Based Maintenance (CBM) in DOD: Are we there yet?" (May 8)

Mr. Hernandez, Managing Director at Global Strategic Solutions LLC, talked about condition-based maintenance from the perspective of the Department of Defense (DoD). $\mathrm{He}$ provided an overview of a DoD study on CBM+/Integrated Vehicle Health Management (IVHM) System Interoperability and Standards followed by an explanation of health-ready levels (documented in SAE Standard JA6268), the applicability of PHM to smart manufacturing, and the common challenges/opportunities. The DoD study was performed for the Deputy Assistant Secretary of Defense (Maintenance Policy and Programs) and resulted in the definition of a common IVHM architecture framework.

\begin{abstract}
Examples from the commercial aviation and automotive industries show that there are three elements to consider for PHM: the sensor (asset), transfer of data from the asset, and the use of the data. The automotive and aerospace industries have some of the most sophisticated, integrated vehicle health management (IVHM) capabilities today, but actual practice of condition-based maintenance is lagging (Figure 4 shows the state of IVHM Capability Levels vs. System Sophistication in those industries).
\end{abstract}

The common challenges and opportunities for PHM relate to issues of data ownership, connectivity, and data quality. Standards can address connectivity (e.g., non-integrated data supply chain) and data quality (e.g., inconsistent/non-continuous data collection, and dissimilar preservation of data source context).

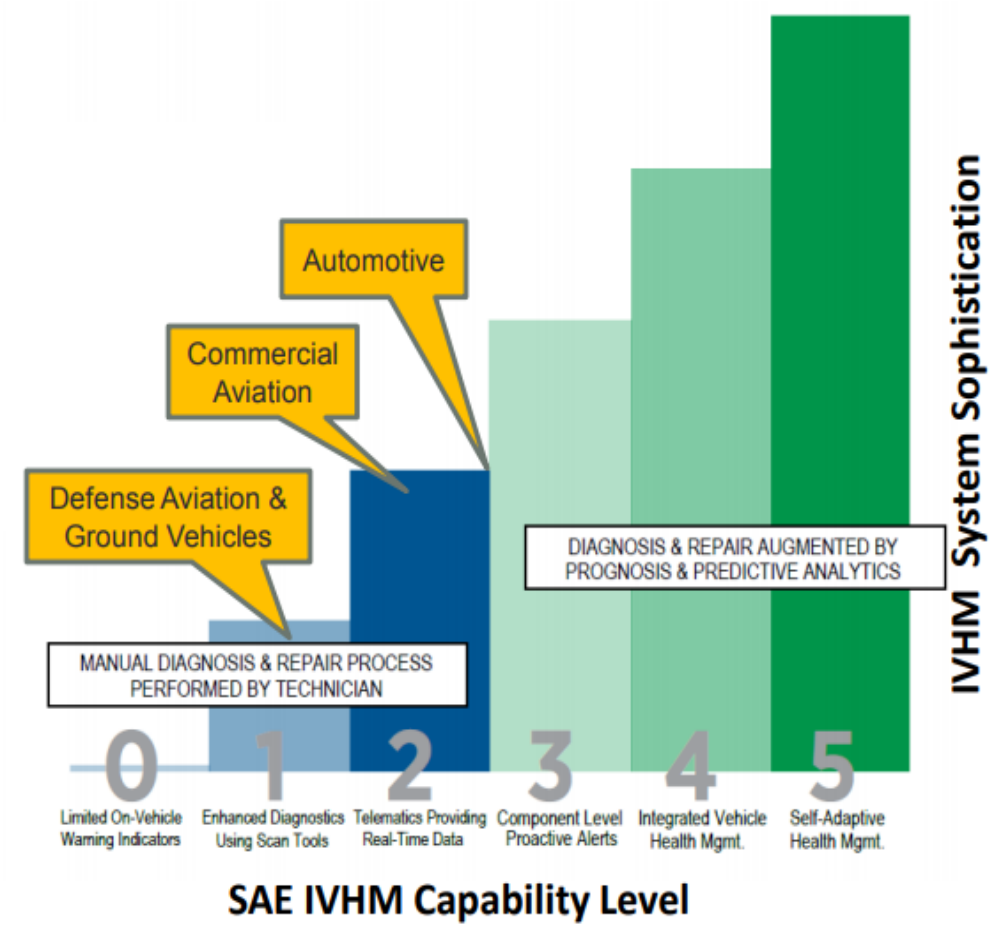

Figure 4: Description of IVHM Capability Level 


\subsubsection{Michael Armacost (Applied Materials)}

"Perspectives on Semiconductor Industry PHM" 9 (May 8)

Mr. Armacost provided an overview of PHM in the context of what is done in the semiconductor manufacturing industry. The complexity of semiconductor chipmaking, which requires around 600 individual steps, is a major factor. The semiconductor manufacturing industry operates under tight tolerances and requires that diagnostics be considered at the individual equipment level. Semiconductor manufacturing equipment is also complex, requiring significant human involvement to obtain diagnostics. Each step in a process affects the next and is interconnected at the factory-level. Both approaches are important. Subject matter expertise with the equipment is critical when analyzing data in semiconductor manufacturing. This was initially a challenge for Applied Materials as they were scaling operations. Challenges also arose in attaining proprietary data and sharing of data. Opportunities exist to perform analytics at every step of the semiconductor life cycle, from production to maintenance. NIST could potentially play a role in getting stakeholders to share data, house that data in one place, ensure data security, and support the development of standards for data partitioning.

Panelists, including James Moyne (Applied Global Services), held a Q\&A panel session. Dr. Moyne is a collaborator of Mr. Armacost. Table 4 provides a summary of the results.

Table 4 - Q\&A - Large Manufacturing Needs and Case Studies Panel

\begin{tabular}{|l|l|}
\hline Question: & Are you requesting preventive maintenance data from suppliers? \\
\hline Answer: & $\begin{array}{l}\text { At Boeing, we ensure control systems on the machines have the proper } \\
\text { tools. The problem is not having the right data, but asking for data tags. } \\
\text { The supplier is often more familiar with specific data. Better } \\
\text { communication between the supplier and manufacturer is needed. }\end{array}$ \\
\hline Question: & $\begin{array}{l}\text { How much enforcement is there of PHM policies? Is there a } \\
\text { penalty? }\end{array}$ \\
\hline Answer: & $\begin{array}{l}\text { In our case (Global Strategic Solutions), there is Office of the Secretary } \\
\text { of Defense (OSD) policy which follows a handbook. There is no penalty } \\
\text { for not following the policy, but the armed services are following it. }\end{array}$ \\
\hline Question: & $\begin{array}{l}\text { Are you looking at use cases to improve yield? What do you consider } \\
\text { a reasonable improvement? }\end{array}$ \\
\hline Answer: & $\begin{array}{l}\text { Applied Global Services is not looking at use cases to improve yield. } \\
\text { One technique under examination is virtual metrology in which we have } \\
\text { uniformity requirements down to the nanometer. This helps to } \\
\text { understand the requirements across the wafer, predict requirements, and } \\
\text { make necessary adjustments. }\end{array}$ \\
\hline
\end{tabular}

\footnotetext{
${ }^{9}$ Michael Armacost presented in place of James Moyne, Applied Global Services, a change from the agenda. Both participated in the panel Q\&A session.
} 
Table 4 (cont'd) - Q\&A - Large Manufacturing Needs and Case Studies Panel

\begin{tabular}{|c|c|}
\hline Question: & Can you share how Boeing recertifies materials? \\
\hline Answer: & $\begin{array}{l}\text { Opportunities exist to recertify rather than discard composite materials } \\
\text { to extend the lifecycle. The opportunity for recertification of composites } \\
\text { is three times greater than for other materials. }\end{array}$ \\
\hline Question: & $\begin{array}{l}\text { Can subject matter experts (SMEs) with knowledge of the system } \\
\text { handle data analysis? }\end{array}$ \\
\hline Answer: & $\begin{array}{l}\text { At Boeing, we encourage a team approach. Those doing data collection } \\
\text { should know the infrastructure and standards for the data collection. } \\
\text { Some machines come with standards built-in, while others do not } \\
\text { Bringing in data scientists early is best. Information Technology (IT) } \\
\text { experts conduct the system integration. However, coordinating } \\
\text { responsibilities from different groups is difficult to manage in a large } \\
\text { company (corporate silos). Communication needs to be transparent. }\end{array}$ \\
\hline Question: & What is the relationship between sensors, analytics, and experts? \\
\hline Answer: & $\begin{array}{l}\text { Sensors can improve the life of equipment. We need to invest in } \\
\text { technology and then apply that technology to other areas. Sensors drive } \\
\text { the industry; analytics are secondary. Knowing the PHM readiness leve } \\
\text { of components is important. To help with predictive maintenance, we } \\
\text { need SMEs to differentiate between a primary signal and less important } \\
\text { signals. Collaboration between sensors and data experts is very } \\
\text { important. The supplier and integrator need to have back and forth } \\
\text { communication. }\end{array}$ \\
\hline Question: & $\begin{array}{l}\text { Do we need both sensors and multivariable models for predictive } \\
\text { maintenance? }\end{array}$ \\
\hline Answer: & $\begin{array}{l}\text { The approach is to infer phenomena using sensors and expertise, then } \\
\text { make investments to develop models based upon the phenomena } \\
\text { Multivariable models are valuable, but the subject matter expertise is } \\
\text { most important. Customers use equipment in a variety of ways, and there } \\
\text { is insufficient data to interpret failure modes on data alone. Processes } \\
\text { also change every year (or more frequently) as the industry evolves. }\end{array}$ \\
\hline
\end{tabular}


Table 4 (cont'd) - Q\&A - Large Manufacturing Needs and Case Studies Panel

\begin{tabular}{|c|c|}
\hline Question: & $\begin{array}{l}\text { Can we ask manufactures to deliver health-ready components? Not } \\
\text { necessarily related to the health of the process, but rather the } \\
\text { equipment. Is the health-ready component } 90 \% \text { of the solution, or } \\
\text { is there too much focus on the component and not the process? }\end{array}$ \\
\hline Answer: & $\begin{array}{l}\text { The problem is that the suppliers of a component keep making } \\
\text { improvements, but do not communicate these to the integrators. There } \\
\text { is a need for a process to determine how 'PHM ready' a component is. } \\
\text { In the semiconductor industry, cryopumps are expensive and fail quite } \\
\text { often. The pump can operate properly in terms of pumping capacity; yet } \\
\text { could be pumping too many particulates that ruin the product. The } \\
\text { operator just wants to receive the data, which they can interpret based } \\
\text { upon subject matter expertise. Suppliers seldom offer useful data; } \\
\text { standards to aid suppliers in giving integrators data could be useful. } \\
\text { Equipment also needs to adhere to certain criteria. } \\
\text { An example use case for collaboration involves GE and Boeing. GE } \\
\text { could provide Boeing with jet engine data; the integrator could then sell } \\
\text { an entire integrated process. The need is for top-down standards that } \\
\text { facilitate understanding relationships between equipment and processes } \\
\text { for specific applications. }\end{array}$ \\
\hline Question: & As automation advances, how can PHM tie in with safety? \\
\hline Answer: & $\begin{array}{l}\text { Safety has many applications: human safety, product safety, equipment } \\
\text { safety, etc. Prognostics applies to all of these. Human safety requires a } \\
\text { higher level of certainty than other applications. It is important that } \\
\text { equipment suppliers build safety into the model. It requires adding a new } \\
\text { specification dedicated to safety. There is a high degree of readiness for } \\
\text { PHM as it relates to safety; it should be part of the control function. }\end{array}$ \\
\hline
\end{tabular}

\subsection{Small to Medium Manufacturing Needs and Case Studies}

\subsubsection{Scott Sipe (Mantec)}

“The Connected Factory for SMMs - Opportunities and Challenges" (May 8)

Mr. Sipe provided a company overview showing the diverse industries, including the small to medium manufacturers (SMMs), that Mantec has worked with in southern Pennsylvania. Mantec visits 140 companies, on average, every year and collaborates on hundreds of projects. Numerous counties in Pennsylvania, including Adams, Franklin, Lebanon, and York, contain 'big industry' manufacturers. A few of these companies are Utz, Harley Davidson, and Armstrong.

Many of the companies Mantec works with are multi-generation-owned companies that are slow to evolve. It is difficult to collect data because much of the work exists in silos or information takes the form of tribal knowledge; tribal knowledge is critical with SMMs. Even if this information is captured, it is usually not well-documented. Mantec helps identify and provide the information SMMs need to make decisions. In many cases people 
may be trained on Lean Automation practices, yet lack enough information to get ahead of maintenance. Downtime is detrimental for small/medium manufacturers.

A few case studies demonstrate where Mantec assisted companies to achieve predictive maintenance. Utz, a food manufacturer, was able to monitor equipment in real-time using RedZone software. Poolpak, a pool dehumidification company, developed a predictive maintenance model with on-line data logging, and remote troubleshooting and adjustments. Mantec is also developing their own smart manufacturing cell with robotics, in collaboration with one of their industry partners, to serve as an additional case study.

\subsubsection{Tom Zbell (Genedge)}

"NIST MEP Industrial Forum Monitoring, Diagnostics, and Prognostics for Manufacturing Operations" (May 8)

Mr. Zbell emphasized that small manufacturers do not typically have preventive maintenance (PM) programs with the exception of some small chemical and printing house manufacturers. These small industries typically run equipment until it breaks instead of PM programs. The most pressing need for small manufacturers are resources to address simple preventive maintenance tasks. The most sought-after resources are those personnel who have the time and knowledge to perform maintenance tasks. Second to this resource need is either having (in inventory) or acquiring the required spare parts.

Medium-sized facilities practice more advanced PM but still lack inter-connectivity between equipment. Some sensors and monitoring equipment may be in place, but these are typically standalone and not connected. Some challenges for medium-sized manufactures are production output overrides of PM scheduling, lack of time for PM, and difficulty justifying the cost of sensors and monitoring systems, especially on older equipment. It is typically cost prohibitive for an SME to retrofit a legacy system with advanced sensing capability. Instead, there is a greater focus on appropriately specifying new systems.

A "shining example" from an automotive supplier described how an interconnected monitoring system has been developed using Tableau software. Finally, large manufacturers were encouraged to share programmable logic controller (PLC) data with SMMs, as they are not using available data from systems effectively.

\subsubsection{Thorsten Wuest (West Virginia University)}

"Smart Manufacturing for SMMs: Opportunities and Challenges" (May 8)

Dr. Wuest presented his vision of smart manufacturing, reported on the results of a smart manufacturing survey conducted at West Virginia University, and described three case studies with recommendations. Smart manufacturing principles involve connectivity, virtualization, and data utilization. More specifically, he views technology, data, and human ingenuity as the smart manufacturing marriage. Small plants do not have the capabilities of multi-national corporations. However, the potential exists to upgrade current 
systems to make them more smart manufacturing-ready. Applying new capabilities such as condition monitoring can help to prevent unplanned downtime.

The results of a survey of West Virginia industries illustrated awareness of smart manufacturing and need to achieve "smarter" manufacturing operations at their facilities. Barriers identified to integrating smart manufacturing at small to medium enterprises include lack of opportunity, lack of resources, lack of a skilled workforce, and high costs.

Three small case studies included a boiler revision project, compressed air system monitoring, and smart services project. Recommendations to help small and medium enterprises included the need for education, success stories, strong sustainable partnerships between industry, academia and industry associations, and ability to leverage state and federal funding.

\subsubsection{Mark Walker (D2K)}

“Crafting Intelligent System Management Using Requirements-Driven Design” (May 8)

Mr. Walker presented an overview of PHM, followed by definitions of requirements-driven design, ending with reusing object-oriented (OO) Platforms. He represents D2K Technologies, which is a company aimed at developing and providing solutions to PHM practitioners (e.g., manufacturers). The output reliability-centered design solutions have led to "Situation Aware" software available to end-users, including manufacturers. In discussing PHM, Mr. Walker noted that PHM systems should determine the health of the equipment and process and its impact on system functions, as well as monitor early warning signs of failure. Currently, PHM is expensive and resource-intensive, can take time to deploy, is often an afterthought, and can include excruciating testing and validation cycles.

Integrating PHM into the design phase is important, and the tools and analyses used should support PHM. Failure Modes and Effects Analysis (FMEA), and Probability Risk Assessment (PRA) are other key tools. A process exists to select the best architecture to enable PHM. The goal is to transform data into information and knowledge based on operational context, leveraging all available wisdom. Delivering on a project incrementally should be an emphasis; incremental successes reduce risk as opposed to delivering a large project at once.

Following Mr. Walker's presentation, the panelists held a Q/A panel session to discuss issues relevant to SMM needs and case studies. Table 5 provides a summary of the results.

Table 5-Q\&A - Small to Medium Manufacturing Needs/Case Studies Panel

\begin{tabular}{|l|l|}
\hline Question: & How long do preliminary PHM assessments take? \\
\hline Answer: & Usually the assessment lasts one or two days. \\
\hline
\end{tabular}


Table 6 (cont'd) - Q\&A - Small to Medium Manufacturing Needs/Case Studies Panel

\begin{tabular}{|c|c|}
\hline Question: & $\begin{array}{l}\text { How do you determine what to include in a walkthrough assessment to add/augment } \\
\text { PHM capabilities within an operation? }\end{array}$ \\
\hline Answer: & $\begin{array}{l}\text { This question references an example provided where a boiler died overnight delaying } \\
\text { production by } 2 \text { hours in the morning before it came back online. This is an excellent } \\
\text { example highlighting a need for greater PHM to raise awareness of impending and } \\
\text { realized faults and failures. From the perspective of a PHM integrator, if this type of issue } \\
\text { (e.g., boiler's failing overnight), is presented during the walkthrough with a customer, it } \\
\text { would be included in the PHM assessment. Continuing with this specific example, data } \\
\text { loggers, from the customer, participated in the walkthrough. As the walkthrough } \\
\text { continues, the manufacturer reports issues of note with equipment and/or processes. In } \\
\text { this example, this include measuring relevant data from compressors and other equipment. } \\
\text { Issues identified are included in the report and recommendations are made (e.g., putting } \\
\text { timers on equipment precludes the need for employees to come in early for preheating). }\end{array}$ \\
\hline Question: & $\begin{array}{l}\text { Do you see greater gains in efficiency when incorporating human ingenuity? How } \\
\text { have you incorporated human ingenuity through your work? }\end{array}$ \\
\hline Answer: & $\begin{array}{l}\text { We are ready to apply techniques to predict an event and it is critical to know what the } \\
\text { customer wants - typically, how to run a smart manufacturing plant. They have the human } \\
\text { ingenuity (knowing how their specific plant works). Our expertise as a developer of smart } \\
\text { manufacturing software combines with their knowledge of the plant to find solutions. } \\
\text { Good communication is important. Workers are scared of "smart" because they think it } \\
\text { means they will lose their job. Communicating that "smart" will help workers perform } \\
\text { their job more effectively and efficiently is essential. This is a gap to address. Creating an } \\
\text { incremental approach at small cost is ideal. Capability will also bring about opportunity. }\end{array}$ \\
\hline Question: & $\begin{array}{l}\text { What is the role for using verifiable tribal knowledge and coupling it with } \\
\text { technological data monitoring? }\end{array}$ \\
\hline Answer: & $\begin{array}{l}\text { Creating a system to shadow people near retirement helps transfer tribal knowledge to the } \\
\text { next generation. A standard to collect this information and transfer it to new workers } \\
\text { would be useful. Tribal knowledge is technical knowledge and 'gut feel' for how a } \\
\text { manufacturing process is performing. Capturing this functional description creates an } \\
\text { opportunity to start standardizing the documentation of the knowledge (e.g., common } \\
\text { terminology). It is hard to replace tribal knowledge with sensors because intuition about } \\
\text { how a process is working is usually a combination of many triggers. Often, the operations } \\
\text { person has the most insight and gut feeling; collecting that feedback is useful. }\end{array}$ \\
\hline Question: & $\begin{array}{l}\text { Do you consider reliability when designing a system? How do you differentiate } \\
\text { between a sensor problem and a component problem? }\end{array}$ \\
\hline Answer: & $\begin{array}{l}\text { A system that measures the reliability of a component, and capability to identify and } \\
\text { design a tolerance for that reliability, is a necessity. The previous performance and } \\
\text { lifecycle of a sensor is a consideration. It might be necessary to add instrumentation to } \\
\text { increase confidence in the system and components. }\end{array}$ \\
\hline Question: & $\begin{array}{l}\text { As small manufacturers, how do you deal with high costs of Information Technology } \\
\text { (IT) and PHM solutions in general? }\end{array}$ \\
\hline Answer: & $\begin{array}{l}\text { (From D2K's perspective) Cost is a big impediment of monitoring and metering. } \\
\text { Sometimes it may not make sense to implement a solution. Using graduate students to } \\
\text { implement a solution at almost no cost is one approach. The decision to invest is } \\
\text { determined by the company strategy, return on investment (ROI), and if PHM is needed } \\
\text { to be competitive. }\end{array}$ \\
\hline
\end{tabular}




\section{Design and Implementation of PHM}

\subsection{Planning and Assessment to Promote PHM Technologies}

\subsubsection{Karl Reichard (Penn State University Applied Research Lab)}

"Driving Requirements for Prognostics - How Far In The Future Do We Need to Predict?" (May 9)

Dr. Reichard discussed setting time requirements for how far into the future prognostics and health management systems must predict failure and provided a background of the Penn State Applied Research Laboratory, which is a Navy University Affiliated Research center. Their mission is to develop and transition technology solutions, enable cost savings for acquisitions, and train the next generation of science, technology, engineering, and math (STEM) students.

Decreasing the prediction time horizon (how far into the future a system must predict health) reduces the uncertainty in health predictions. One approach is to examine the functions that contribute to time horizons and threshold requirements. Failure prediction should occur with enough time to take necessary action.

There are many different applications (e.g., safety, maintenance, life cycle cost, etc.) for health monitoring and management, and all have different requirements. These requirements drive different prognostic approaches. Approaches to PHM influence responsiveness of the PHM system and drive time horizon requirements. Three possible approaches: experience/data-driven, physics-based, and data analytics-based prognostics. A combination of all three can help determine fault time horizons.

\subsubsection{Ananth Seshan (MESA)}

"Learnings - Practical Use Cases in Proactive Asset Performance Management" (May 9)

Dr. Seshan introduced MESA, a nonprofit working with NIST on smart manufacturing solutions. He discussed the need for, and presented the concept of, an 'Asset Intelligence System" - an intelligent software platform that allows for seamless bi-directional collaboration between the plant floor and the enterprise. More specifically, the Asset Intelligence Systems' function was defined as follows: (a) real time monitoring of asset data, (b) conversion of the real time asset data into meta-data or intelligence (on potential failures or degrading health of equipment that can be actioned upon), and finally, (c) generation of appropriate actions (e.g., work orders) in real time by means of a seamless vertical integration to enterprise asset management systems to prevent failures. Eight use case scenarios that are active today in the field were presented to demonstrate the Asset Intelligence System.

The presentation included details on how the Asset Intelligence System can generate proactive reports, opportunistic work orders to prevent failures, useful information on root cause of failures, and can leverage data (in some cases) to predict machine failures. 


\subsubsection{Kai Goebel (NASA)}

"Designing Resilient Engineered Systems with PHM" (May 9)

Dr. Goebel defined resilience as the ability of a system to "spring back" when faced with a fault. During the design phase of a system, one should consider both PHM as one of several possible mechanisms that accomplishes resilience. A proposed engineering design framework was presented that would be agnostic to any design and work with any complex engineered system (CES).

Definitions included different levels of PHM sophistication, from "let it fail" to "selfhealing." Understanding resilience metrics is important to quantify and measure the impact of a fault, which helps determine the required level of PHM sophistication. Operational life-time simulations of different design candidates allow the choice of the most resilient design solution. Integration of PHM at different levels could then be done in an informed manner such that operational metrics are maximized.

\subsubsection{Miguel Saez (University of Michigan)}

"Modeling and Analysis of Cyber-Physical Manufacturing Systems for Anomaly Detection" (May 9)

Dr. Saez introduced cyber-physical manufacturing systems as an interaction between physical components (e.g., sensors, dynamic models, part information), software (e.g., control logic, commands and algorithms), and networks (e.g., DeviceNet, Ethernet/IP, and Internet of Things). The main objective of the systems from a PHM perspective is to improve anomaly detection and diagnosis in manufacturing processes. Model cyberphysical systems that consider both cyber and physical domains while being contextspecific enables the objective. Data/information should merge with expert knowledge.

Notably, feature extraction of a non-stationary signal can improve by adding information from the cyber domain. Modeling in general requires merging expert knowledge and machine data into process analysis algorithms. To support this effort, a case study was conducted on automotive body-in-white conveyor system.

\subsubsection{Jorge Arinez (General Motors (GM))}

"Planning and Assessment to Promote PHM in Automotive" (May 9)

Dr. Arinez provided a high-level perspective of PHM from the perspective of the automotive industry, which deals with great variability, complexity, and short marketplace duration for some products. GM's Global Research and Development operations are located in Michigan and focused on inventing and commercializing innovative technologies to enhance automotive manufacturing and products. GM is beginning to leverage real-time data from within the factory to deal with challenges of complexity and variability. The goal is to move towards more systems-integrated production and maintenance operations. As automation expands, how automation interacts with people becomes more important. 
Continuous improvement is a major challenge as sudden changes in operating conditions occur. One method for continuous improvement is opportunistic maintenance (e.g., maintenance when a process is already down for an unrelated reason). Maximizing throughput should always be a factor when considering maintenance.

Standards are important for consistent terminology and communication between applications. Standards can also assist in the data cleaning process. Advanced analytical tools are necessary due to the tremendous amount of data exchange to and from devices, processes, and equipment in the factory. To increase adoption, future standards should be meaningful, relevant, easy to apply, and offer flexibility to the manufacturing community.

Panelists held a Q/A panel session to discuss issues relevant to planning and assessment to support PHM. Table 6 provides a summary of the results.

Table 6 - Q\&A - Planning and Assessment to Promote Monitoring, Diagnostics and Prognostic Technologies

\begin{tabular}{|l|l|}
\hline Question: & $\begin{array}{l}\text { How does a methodical analytical and statistical approach compare to a } \\
\text { subject matter expert's knowledge? }\end{array}$ \\
\hline Answer: & $\begin{array}{l}\text { The methodological approach allows you to go through different solutions. The } \\
\text { SME will incorporate what they know, which could be a benefit or a limitation. } \\
\text { Designing optimization modules at the front-end allows assessment of many } \\
\text { alternative approaches automatically. Having these statistical results and } \\
\text { reported changes exceeds what a person can generate from self-knowledge. }\end{array}$ \\
\hline Question: & $\begin{array}{l}\text { As PHM levels increase, the ultimate goal is to achieve a self-healing system. } \\
\text { How do you envision the interaction between man and machine in a self- } \\
\text { healing system? }\end{array}$ \\
\hline $\begin{array}{l}\text { More PHM is not necessarily better. PHM for low impact operational equipment } \\
\text { is not necessary. A more methodical approach can guarantee a positive return on } \\
\text { investment when actually implementing PHM. All of the tools and analytical } \\
\text { approaches are case- and skill-specific. Line workers have a certain level of skill } \\
\text { and sophistication that differs from engineers, managers, and executives. } \\
\text { Industry does not have the luxury of Ph.D.'s to analyze all of the information. } \\
\text { Examining the sophistication of the tools allows us to deploy and match them } \\
\text { with the skillset of the people that will use them. }\end{array}$ \\
$\begin{array}{l}\text { It is important to ensure all steps are as automated as possible so the only thing } \\
\text { the operator gets is a maintenance schedule. Automated collection and analysis } \\
\text { of data and output into work orders is efficient; it can potentially even enable } \\
\text { setting of priorities. }\end{array}$ \\
$\begin{array}{l}\text { Original Equipment Manufacturers (OEMs) should embed sensors in machines } \\
\text { and offer services. Incorporating an augmented reality device such as a wearable } \\
\text { heads-up display allows you to stream a maintenance issue to a remote expert. } \\
\text { This includes a mix of human and automation/machine interaction. A human is } \\
\text { a sensor. When applying machine learning techniques, the challenge is to } \\
\text { achieve accurate and repeatable documentation that is also verifiable. }\end{array}$ \\
\hline
\end{tabular}


Table 6 (cont'd) - Q\&A - Planning and Assessment to Promote Monitoring, Diagnostics and Prognostic Technologies

\begin{tabular}{|c|c|}
\hline Question: & $\begin{array}{l}\text { Large facilities have a lot of equipment and assets. When you experience } \\
\text { faults, how do you reconcile adding PHM capability from 1) in-house, 2) out } \\
\text { of house, or } 3 \text { ) a complete redesign of the process? }\end{array}$ \\
\hline Answer: & $\begin{array}{l}\text { Every time there is a facility redesign, we bring in the latest and greatest } \\
\text { technology, so a combination of internal and external expertise is used. } \\
\text { Standards have a role in this process. We analyze specifications when we acquire } \\
\text { equipment. Having a standard, consistent way to do this is vital to comparing } \\
\text { one piece of equipment to another. }\end{array}$ \\
\hline Question: & How difficult is it to integrate PHM across a whole facility? \\
\hline Answer: & $\begin{array}{l}\text { It is a challenge to integrate PHM vertically. When to replace a machine is not } \\
\text { just dependent on health, but also on quality, productivity, and other } \\
\text { performance factors. Having a standard defining how to integrate PHM both } \\
\text { vertically and horizontally would be valuable. The temptation is to fix the } \\
\text { problem occurring right now. It is important to take a step back and see what the } \\
\text { process should accomplish and what requirements would enable that goal. }\end{array}$ \\
\hline Question: & $\begin{array}{l}\text { Today's state of Manufacturing } 4.0 \text { integrates an ecosystem (e.g., OEMS, } \\
\text { suppliers, etc.). Do we need a unified approach or standard that addresses } \\
\text { the entire ecosystem? }\end{array}$ \\
\hline Answer: & $\begin{array}{l}\text { OEMs have tried to drive standards, but this typically fails. In the past, we have } \\
\text { let the supply base evolve organically. The preference is not to engineer our own } \\
\text { solution so system A can talk to system B; it would happen too slowly. The } \\
\text { industry needs a common communication protocol that works throughout the } \\
\text { supply chain. Closing the gap across the ecosystem should occur incrementally; } \\
\text { standards must be developed and adopted incrementally, as well. A standard } \\
\text { addressing quality would be a good starting point for the incremental approach. } \\
\text { Historically many standards have been interface-centric. The industry does not } \\
\text { want standards to require that manufacturers purchase specific equipment. As } \\
\text { the scope expands from single machines to the whole factory, we have to identify } \\
\text { where to set boundaries for standards. } \\
\text { National Aeronautics and Space Administration (NASA) has integrated complex } \\
\text { systems and has at times failed. The interface restrictions are strict in this } \\
\text { industry. Software systems and PHM must have clear definitions. }\end{array}$ \\
\hline
\end{tabular}

\subsection{Emerging Sensing Technologies to Enable PHM}

\subsubsection{Radu Pavel (TechSolve)}

“An MTConnect ${ }^{\circledR}$-Based Approach for Machine Monitoring” (May 9 Panel)

Dr. Pavel introduced TechSolve, Inc. - an organization based in Cincinnati, Ohio, providing business advisory, machining process solutions, and IoT solutions. TechSolve performs research and development $(\mathrm{R} \& \mathrm{D})$ that industry normally would not pursue because of limited expertise, lack of instrumentation, and impact on production operations. TechSolve's definition of a PHM system for manufacturing assets was presented as an 
integrated health monitoring system capable of accurately monitoring and predicting the machine and process health for near-zero downtime, minimal scrap, and high quality.

TechSolve's PHM research includes a focus on the machine tool as an overall system of systems. It is important to understand the health in all machining subsystems including the machine tool elements (spindle, feed axes, fluid etc.), cutting tool, work material, cutting fluid, machining accessories, and machining parameters. A challenge is to avoid clogging a system network with too much data from multiple sensors. Using an adapter with edge computing instead of a generic data collection program assists with extracting only the features deemed meaningful for monitoring, diagnostic, and prognostic tasks. Dr. Pavel presented current efforts in PHM for machine tools using an MTConnect@-based adapter with edge computing, providing a sample monitoring system architecture, evaluation and validation tests, and data analytics. In support of their R\&D activities, TechSolve instrumented machine tool test-beds, and spindle and feed-axis test-beds that output multiple data sources including vibration, temperature, power, displacement, speed, feed rate, load, and other parameters.

\subsubsection{Brittany Newell (Purdue University)}

"Industrial Capacitance Sensors and Actuators" (May 9 Panel)

Dr. Newell focused on recent research and emerging technologies related to sensors. The goal of her current efforts is to provide an in-line and minimally invasive means for monitoring industrial component health to prevent catastrophic disasters and a method for at least temporarily mitigating failure. The expected impact of this work is to increase operator safety and cost savings while minimizing unplanned downtime.

Part of the presentation described how capacitive sensing works and how it contributes to detecting failures. Industrial applications of capacitive sensing are detecting failures in hydraulic hoses, tires, O-rings/seals, and v-belts. Part of Dr. Newell's research is building capacitive sensors into hoses to offer direct health monitoring of this specific part.

Electroactive polymer industrial materials provide a good demonstration of actuation. Prestraining materials enhances the actuation. Experiments yielded a displacement of $1 \mathrm{~mm}$ when the polymer material was pre-strained. The electroactive polymer construction reveals the measured response capability. Ultimately, Dr. Newell's work yielded the ability to sense device health across a variety of industrial components in support of the expected impact.

\subsubsection{Justinian Rosca (Siemens Corporation)}

"Validation of the Intelligent Edge" (May 9 Panel)

Dr. Rosca provided an overview of edge computing and machine learning. Machine learning is essential to enable PHM, as it offers a powerful toolkit for building useful complex prediction systems quickly and cost-effectively. One challenge for machine learning is that the "plumbing" is overwhelming, covering configuration, data collection, 
feature extraction, data verification, and other elements. There are also business components to consider, including engineering, programming, and data management.

One example is edge monitoring and prognostics for high-speed trains. The intelligent edge builds on synchronization of the real (physical, practical) and digital worlds. Maintaining a digital twin over a lifetime is difficult and expensive. Safe, trustworthy machine intelligence is essential from multiple perspectives in deployed systems; standards and collective engagement of many stakeholders is required for predictive intelligence in the edge.

\subsubsection{Gregory VogI (NIST - Intelligent Systems Division)}

"Emerging Sensing Technologies towards Smart Machine Tools" (May 9 Panel)

Dr. Vogl summarized how technology innovations in PHM and smart tools relate to economic growth. Over the past ten years, manufacturing has experienced a resurgence of jobs. The biggest challenge manufacturing faces is unplanned downtime - proactive maintenance can address this. Wear accounts for $65 \%$ of equipment failure, and most failures are attributable to mechanical failures, rather than chemical. Excessive wear causes a part to exceed the quality tolerance.

Many manufacturers claim that routine tracking of performance is too expensive. The goal is to develop inexpensive tools for smart machines that enable online (i.e., networked together) and data-rich measurements. The machine tools must be self-aware with diagnostics of performance and root causes of incipient faults and failures. They must enable the prediction of part errors based on health tracking and optimize asset management. One specific research goal is to enable "machine health in five minutes or less."

Examples of work performed at NIST include projects to help enable smart machines, such as a linear axis testbed highlighting the root cause of rail wear. Lessons learned through this work include the need for traceable, dimensional (related to physical quantities) and verifiable data; simpler analytics and user set-up should be streamlined.

Dr. Vogl's slide deck is included in Appendix D - NIST Presentations.

\subsubsection{Ed Spence (Machine Instrumentation)}

"Machine Health for the Machine Maker" (May 9 Panel)

Mr. Spence made the case for why sensors are important. From his perspective, four technologies make up the Industrial Internet of Things (IIoT) landscape today, including chip scale sensors, predictive analytics, wireless networks, and data dashboards. Sensors play a role in all of these technologies. Third party service providers or plant maintenance perform much of today's sensing manually.

An opportunity exists for the OEM/machine maker to embed condition-based maintenance (CBM) sensors within the equipment. They also can develop the digital interface to a local 
controller, customize health indicators, and automate the collection of 'tribal knowledge.' This will help mitigate effects of a retiring workforce and enable leaner maintenance with more cost-effective solutions. This effort would also extend the smart mobile culture, making information easier to access and expand fault coverage.

New measurands require new sensors. Techniques for expanded health monitoring are multi-faceted, and industry should leverage contractors versus developing everything inhouse to accelerate time-to-market. Subject matter expertise can assist with capturing domain knowledge. The first step is for the OEM to begin embedding sensors.

Following Mr. Spence's talk, panelists held a Q/A session to discuss issues relevant to emerging sensing technologies to support PHM. Table 7 provides a summary of the results.

Table 7 - Q \&A - Emerging Sensing Technologies to Enable Monitoring, Diagnostics, and Prognostics

\begin{tabular}{|c|c|}
\hline Question: & $\begin{array}{l}\text { How do you convince SMMs that the MTConnect®-based approach is better } \\
\text { than lean maintenance practices and encourage them to take this technological } \\
\text { step? }\end{array}$ \\
\hline Answer: & $\begin{array}{l}\text { One way is organizing smart manufacturing seminar scenarios, where small and } \\
\text { medium manufacturers attend and learn. We can communicate successes via low } \\
\text { cost pilot projects, including case studies/success stories. Companies can then } \\
\text { discover their machine utilization is not as high as it could be. Consultants could } \\
\text { also work with academia to show case studies and demonstration cases. }\end{array}$ \\
\hline Question: & $\begin{array}{l}\text { Are you more concerned about health of individual equipment or integrated } \\
\text { productivity? }\end{array}$ \\
\hline Answer: & $\begin{array}{l}\text { If you have an idea of tools utilization from each machine, a business intelligence } \\
\text { approach can determine how to optimize the whole system at the plant level. We } \\
\text { were working on analytics for this. Data management systems for tool utilization } \\
\text { are better when used with integrated data. } \\
\text { Be careful using low cost sensors because the system it runs on might be more } \\
\text { expensive. Sensors might not be high enough quality for industrial applications. } \\
\text { Expensive sensors typically produce less noise. } \\
\text { Collecting data is the first step to asset management, so measure at the machine } \\
\text { level. Integrating a software tool for asset management is the next step. It is up to } \\
\text { the industry to create the path of this technology. }\end{array}$ \\
\hline
\end{tabular}

\subsection{Monitoring and Analysis Technologies for PHM}

\subsubsection{David Siegel (Predictronics)}

"We Know What Happens Next: Perspectives and Case Studies on PHM Technologies for Manufacturing" (May 9 Panel)

Dr. Siegel provided an overview of Predictronics and contributions on predictive analytics solutions for PHM technologies in manufacturing industries. This included general 
examples of PHM applications, common assets, and related problems in manufacturing sectors. Predictronics provides custom predictive monitoring and analytical software, mainly for industrial applications. Four common application domains that Predictronics' customers have identified as maintenance issues are 1) machine tool predictive monitoring, 2) industrial robots, 3) stamping machines, and 4) diecasting operations. Predictronics' software is capable of offering enhanced intelligence regarding the health of the aforementioned processes and equipment.

An example of the predictive software they offer is machine tool predictive monitoring (e.g., spindle, linear axis, tool wear, etc.). The purpose of the tool is to provide useful and digestible information for shop floor employees; this information is valuable for education, but also beneficial in identifying predictive maintenance opportunities. The tool has a datadriven methodology for planning the frequency of scheduled maintenance. Another example is Predictronics' development of a robot health index based upon joint angle, velocity, and current captured from individual robot controllers. Predictronics is using this information to help the customer qualify the health of the robot and/or process the robot is contributing.

Future work includes recommending and developing standards. An obvious need exists for standards for these tools, as well as other future technologies such as industrial robots and supporting communication protocols (e.g., further advancement of the MTConnect@ standard). Additional standards that would benefit the community would be the generation of guidelines to verify and validate manufacturing PHM implementations. Likewise, there is a need to develop additional use cases and reference data sets for the community to leverage in further developing predictive capabilities. Dr. Siegel identified NIST as the likely candidate to address these specific needs given their research focus and neutrality.

\subsubsection{Nancy Diaz-Elsayed (University of South Florida)}

"Production Monitoring for Performance and Energy Efficiency Improvements" (May 9 Panel)

Dr. Diaz-Elsayed introduced several manufacturing challenges during her presentation (e.g., lack of collaboration across departments, return on investment (ROI), justification for investment in improvements, etc.), and cyber-security concerns for monitoring and automation in industrial environments. Examples of manufacturing improvements include connecting products, plant systems, and machines for asset management practices, and developing monitoring and asset management software. Energy modeling for these systems can support prognostics and health management. In the $\mathrm{CNC}$ machine tool example provided, power usage can serve as an indicator for performance. Characterizing and predicting power demand gives additional insight into monitoring machine health. The example applied machine learning to estimate the power demand of machine tools (e.g., by using artificial neural networks and regression models), and future work that was suggested includes training models for process monitoring. 


\subsubsection{Sanket Amberkar (Falkonry)}

"Predictive Analytics Approach with Time Series Data using Machine Learning" (May 9 Panel)

Mr. Amberkar presented on predictive digital analytics using machine learning across key business sectors and provided an overview of Falkonry. Falkonry's business objective is to teach their customers how to find hidden information in their data so they can make smarter maintenance and control decisions. More specifically, the motivation for Mr. Amberkar's work includes increasing productivity improvements and applications of operational machine learning with time series data. The goal is to use machine learning as a crucial tool for insight into time series data. Operational machine learning is the intersection of business software, machine learning, and data analytics. The output of machine learning algorithms can be used to predict what faults and failures will occur and offer actionable intelligence to promote an appropriate response at the ideal time horizon. Falkonry's software has a similar modeling structure to Matlab ${ }^{10}$, where it determines and identifies temporal patterns, which can be conveyed during user notification. An example was presented using the software to find an electromagnetic field (EMF) pattern six days before failure of a robotic arm. This was the result of a Kawasaki motor burning out before its lifetime. The software looks at the precursory pattern before the fault happens, and then feeds the learned data from one machine into other similar machines within the larger system (as defined by the factory or organization). In summary, Falkonry has created a "Data Scientist in a Box" to offer its consumers self-service of Machine Learning tools. This software is applicable to multiple use cases across numerous industries, not just within manufacturing operations.

\subsubsection{Robert Gao (Case Western Reserve University)}

"Stochastic Modeling for System Remaining Life Prognosis" (May 9 Panel)

Dr. Gao provided an overview of the modeling approaches and methods for monitoring the manufacturing systems, which includes the inference, tracking, and prognosis of mechanical manufacturing machines and parts. The goal of this work was to improve the observability of complex systems. Probability density functions (PDFs) give an analytical representation of the sensor measurement, current part status, part state propagation, and future part status for these systems, to account for uncertainties. The particle filter (PF) technique predicts the system performance degradation over time, as well as determines the remaining useful life (RUL) of the system with respect to predicted in terms of PDFs. Improvements to the PF-based modeling technique enhance the accuracy for detecting degradation tracking and part life prediction. This approach, coupled with an experimental setup, can assist prognosis in the future. Connecting the physical world with the cyber world is the best path for predicting system health in the foreseeable future.

\subsubsection{ChaBum Lee (Tennessee Tech University)}

"On-Machine Dimensional Measurement Technology for Prognostics and Health Monitoring for Precision Manufacturing Systems and Processes" (May 9 Panel)

\footnotetext{
${ }^{10}$ https://www.mathworks.com/products/matlab.html
} 
Dr. Lee provided an overview of on-machine metrology (OMM) tools, as well as the measurements collected from those tools (e.g., form error, cutting tool wear, cutting temperature, machine vibration, etc.). There is an extensive need for developing tools to support OMM. Dr. Lee sees value in integrating manufacturing and measurement together where it becomes a closed loop process. Current research on machine surface measurements using OMM is underway. A challenge with this process is having the ability to measure surfaces with complex geometries. It is crucial to have low cost OMM tools to measure important PHM metrics for complex geometric parts. Integrating management systems into machine tools is an important outcome of this research. The motivation to use OMM tools is to define damage size by identifying fringe patterns and designing curve edge sensors that are not sensitive to the materials.

A Q\&A session followed the panel on monitoring and analysis technologies. Table 8 provides the results of this session.

Table 8 - Q\&A - Panel on Monitoring and Analysis Technologies for Prognostics and Health Management

\begin{tabular}{|l|l|}
\hline Question: & $\begin{array}{l}\text { Some data is structured, and some is unstructured. How structured } \\
\text { should the data be? }\end{array}$ \\
\hline Answer: & $\begin{array}{l}\text { There may be gaps in the data that require sampling. You could have } \\
\text { qualitative data (different types), or there may be a point at which you do } \\
\text { not have enough data for an accurate sampling. In that case, you increase } \\
\text { the sample volume or the duration of collection needs to be increased. }\end{array}$ \\
\hline Question: & What is the real definition of a data scientist? \\
\hline \multirow{3}{*}{ Answer: } & $\begin{array}{l}\text { A data scientist 1) wrangles data (leveraging tools to find mathematical } \\
\text { trends, a task not commonly performed by just data scientists), and 2) } \\
\text { performs mathematical correlation. If Artificial Intelligence is needed, } \\
\text { more computer science comes into play. What it comes down to is how to } \\
\text { structure the data and make inferences. Another definition of a data scientist } \\
\text { is someone who identifies how to apply algorithms and machine learning } \\
\text { to solve an industrial problem. Aspects of data science sometimes lack clear } \\
\text { definition. }\end{array}$ \\
\cline { 2 - 2 }
\end{tabular}


Table 9 (cont'd) - Q\&A - Panel on Monitoring and Analysis Technologies for Prognostics and Health Management

\begin{tabular}{|c|c|}
\hline Question: & $\begin{array}{l}\text { What data is critical for PHM that manufacturers find difficult to } \\
\text { collect? What data is hard to verify? }\end{array}$ \\
\hline Answer: & $\begin{array}{l}\text { Sensors are first required to capture the physics and then convert the } \\
\text { captured phenomena to a signal. This is accessible today by leveraging } \\
\text { technology. Second, access to the data is needed, which is the bigger } \\
\text { challenge. For example, there may be restrictions as to placing sensors due } \\
\text { to extreme high temperatures or other environmental conditions. } \\
\text { Another challenge is missing the context to explain the data. There is a } \\
\text { tradeoff between using existing sensors versus adding additional external } \\
\text { sensors, which could be costly. It is difficult to know the solution for a } \\
\text { sensing problem before working towards it. } \\
\text { Our ability to get data from external sensors needs expansion. A range of } \\
\text { sampling frequencies is available, but could cause the machine to be } \\
\text { unavailable for several hours. One solution is clip-on sensors (only } 15 \text { min } \\
\text { of downtime to install). Placing sensors close to the function is ideal; } \\
\text { building PHM capabilities into the design phase is also important. The } \\
\text { quality of the data collected is a measure of final success. }\end{array}$ \\
\hline Question: & $\begin{array}{l}\text { What is your perspective on standardization for PHM? What areas are } \\
\text { most suitable for standardization for PHM? }\end{array}$ \\
\hline Answer: & $\begin{array}{l}\text { The immediate need is a standard for communication and standardization } \\
\text { of the data, itself. The need for machine specific standards is not as urgent } \\
\text { For example, currently quality and maintenance records are not } \\
\text { standardized. Developing reference use cases across equipment would be } \\
\text { helpful, or standardization of measurement technology. Standards for } \\
\text { machines can occur later. There is need for a distributive control model. } \\
\text { You can interchange machines or PHM analytics, and plug and play } \\
\text { interoperability. There is also a need for a platform-based approach. A } \\
\text { modular approach is an eventual objective so reference architecture would } \\
\text { be beneficial. }\end{array}$ \\
\hline Question: & What is the metric for PHM success? \\
\hline Answer: & $\begin{array}{l}\text { Two important metrics are bringing value to the manufacturing community } \\
\text { and enabling manufacturers to reduce costs and become efficient. Overall } \\
\text { equipment effectiveness (OEE) is a metric used by many manufacturers; } \\
\text { productivity, asset availability, and quality are building block metrics that } \\
\text { comprise OEE, when aggregated together. Developing the necessary } \\
\text { measurement science to optimize planned maintenance and minimize } \\
\text { unplanned maintenance can increase the availability of assets. Ideally, we } \\
\text { can eliminate reactive maintenance and optimize preventative and } \\
\text { predictive maintenance. The manufacturing community is going to drive } \\
\text { metrics. }\end{array}$ \\
\hline
\end{tabular}


Table 10 (cont'd) - Q\&A - Panel on Monitoring and Analysis Technologies for Prognostics and Health Management

\begin{tabular}{|l|l|}
\hline Question: & $\begin{array}{l}\text { Given the diversity of products, will there be a PHM standard for } \\
\text { technology or equipment? }\end{array}$ \\
\hline Answer: & $\begin{array}{l}\text { Some standards have a specific focus, while others are more abstract and } \\
\text { closer to guidelines (e.g., ISO 9000); no two implementations are alike. } \\
\text { Industry should drive standards; they should also include a scope that } \\
\text { reaches the majority of manufacturers. Some technologies evolve quickly; } \\
\text { this is a consideration for standards developers (to avoid newly released but } \\
\text { obsolete standards). Manufacturing is becoming more decentralized and } \\
\text { flexible. Developed standards should be flexible enough to adjust to minor } \\
\text { implementations or whole processes, understanding that no two } \\
\text { manufacturers have identical processes. }\end{array}$ \\
\hline Question: & $\begin{array}{l}\text { Data analytics technologies are evolving rapidly. Could we create } \\
\text { standards for domains? }\end{array}$ \\
\hline Answer: & $\begin{array}{l}\text { Yes, if we can get alignment on what the output looks like; the standard } \\
\text { should address decision points for operators. }\end{array}$ \\
\hline
\end{tabular}

\subsection{Communication and Information Flow to Support PHM}

\subsubsection{Will Sobel (Vimana)}

"Health and Maintenance through the Lens of Dynamic Scheduling" (May 8)

Mr. Sobel provided an overview of Vimana and their contributions to PHM technology development via dynamic scheduling software. The company started this project with organizations like DMDII, PARC, and the MTConnect Institute. The health and maintenance of manufacturing systems can be represented using failure rate curves over time, which generally follow a bathtub configuration ${ }^{11}$. However, Vimana is not looking at failure conditions. Instead, they focus on equipment capability and the requirements to manufacture certain parts using that equipment. As expected, certain machines will degrade and then require maintenance. Once the equipment no longer has a certain level of capability, it will require maintenance to restore its capability. This capabilities-based approach enables matching equipment needed to complete a certain process. Conceptually, scheduling can also optimize the number of products being output. Repairing equipment is necessary if it was previously capable of completing a task, that task is still in demand, and that equipment can be reasonably repaired; otherwise the equipment can be retired. Using a milling machine as an example, an old machine may still be useful for roughing, even if it is no longer capable of performing finishing operations.

Standards will provide a common language so systems can communicate their capabilities and status. Vimana is working on modeling these systems and standards. This model will 'pull out' a machine when it can no longer can complete a task, yet has potential for other uses when it is in a relatively degraded state. This will enable the prediction of capability and prescriptive outcomes in the future. Machines could potentially communicate with themselves to eliminate complexities such as global scheduling.

\footnotetext{
${ }^{11}$ https://en.wikipedia.org/wiki/Bathtub curve
} 


\subsubsection{Moneer Helu (NIST Systems Integration Division)}

"Connecting and Deploying Smart Manufacturing Technology to Support PHM" (May 8)

Dr. Helu's research is focused on connecting and deploying Smart Manufacturing technologies across lifecycle phases: design, fabrication, and inspection. The goal is to provide data, physical resources for testing and $\mathrm{V} \& \mathrm{~V}$, education, and demonstration. This research effort incorporates a lifecycle information framework. Current challenges to this methodology include the variety of product lifecycle management (PLM) solutions, primarily information technology (IT) and engineering focused (but expensive), and operations solutions (mixture of IT and operations technology). Integration between operational solutions and PLM solutions is lacking. There are also many outdated operating systems with cybersecurity vulnerabilities.

Leveraging manufacturing data provides further insight into how a facility expects to perform versus its actual operations. There are extensive opportunities for digital technologies to advance manufacturing. A few implementation challenges need to be addressed including data management requirements, implementation costs, and physical environment limitations. These are important next steps for future smart manufacturing deployments.

Dr. Helu's slide deck is included in Appendix D - NIST Presentations.

\subsubsection{Joel Neidig (ITAMCO)}

"Communication and Information Flow to Support PHM" (May 8)

Mr. Neidig provided an overview of Indiana Tool and Manufacturing Companies (ITAMCO) and his organization's contributions to the development of PHM technologies. ITAMCO is a contract manufacturer that conducts R\&D and manufactures parts and systems for private organizations and government agencies (e.g., building radar systems for Raytheon or the National Oceanic and the Atmospheric Administration (NOAA), constructing pillars for offshore rigs). ITAMCO also manufactures large swing gears used in mining equipment for Caterpillar and Komatsu. With ITAMCO's interest in developing PHM technologies, the organization is focused on innovating, including leveraging emerging standards to promote clear and consistent communication throughout their manufacturing operations; MTConnect ${ }^{\circledR}$ is selected as the preferred fit for the company.

One area of interest for ITAMCO is developing tools capable of measuring linear axis diagnostics, just like a ball-bar test. There are simple tools that can get you $90 \%$ of the desired information. An example is using a $\$ 500$ power monitor to observe machine and plant power consumption; these same monitors can be used for air compressors. Energy is a very significant portion of costs. Other examples include a Mazak smart box that can support ten machines (although expensive), and an iBlue ${ }^{\mathrm{TM}}$ system that outputs data to mobile devices. More specifically, iBlue features a Bluetooth module that can connect to a measurement device and wirelessly transmit data to a known recipient(s). The goal is to create self-healing systems. Accomplishing this requires process measurement, which would reduce human error and part replacement due to operating error. 


\subsubsection{Rob Andes (The Design Knowledge Company)}

"Asset Life-Cycle Information Management (ALCIM) Support for Condition-Based Maintenance (CBM)" (May 8)

Mr. Andes provided an overview of The Design Knowledge Company (TDKC), and their efforts in developing software and computer numerical control (CNC) asset instrumentation for asset life-cycle information management (ALCIM) in manufacturing industries. Experiences at the Warner Robins Air Logistics Complex (WR-ALC) Commodities Manufacturing (CMXG) Operations Support for condition-based maintenance (CBM) provided context for the presentation. TDKC has applied Java-based software in all different sectors, both high- and low-tech. To get to integration, the TDKC software needed 17 different data sources (in various forms); focusing on production of Overall Equipment Effectiveness (OEE), they visualized manufacturing scheduling, flow rates, and throughput. A few examples of TDKC technology platforms include monitoring boxes for integrated manufacturing environments and leveraged technologies like MTConnect ${ }^{\circledR}$. A few challenges associated with these technologies emerge, such as the need to be constantly engaged in dynamic manufacturing and requiring prognostics technologies in a three-shift environment.

There are challenges in the ALCIM program, as well as benefits due to the software's simple displays and remote diagnostics monitoring capability. Machine variables are associated with CBM support, such as power management, spindle and motor metrics, and hydraulics. There are machine and environmental challenges to address, such as even with four of the same machine, they have different heartbeats. Solving challenges requires identifying idle configurations and runtime baselines, and comparing this thumbprint to the actual data coming out. Current work is focusing on development of machine learning (ML) algorithms, specifically support vector machines (SVM) to compare machine baseline health data against work-in-progress part manufacturing data to identify subtle vibration anomalies. This helps notify engineers to investigate or not. Solutions that are more elegant will help address the challenges in disconnected, secure environments and anticipate managed machine maintenance cycles using prognostics models.

A Q\&A session followed the panel on communications and information flow. Table 9 summarizes the results of panel discussions, organized by discussion point.

Table 9-Q\&A - Monitoring and Analysis Technologies for Prognostics and Health Management

\begin{tabular}{|l|l|}
\hline Question: & What does dynamic scheduling mean and how long is the time horizon? \\
\hline \multirow{2}{*}{ Answer: } & $\begin{array}{l}\text { The time horizon is } 10-24 \text { hours out. Dynamic scheduling is a continuous } \\
\text { process; as the environment changes, the algorithm will recalculate the optimal } \\
\text { schedule. There is continuous feedback. }\end{array}$ \\
\cline { 2 - 2 }
\end{tabular}


Table 9 (cont'd) - Q\&A - Monitoring and Analysis Technologies for Prognostics and Health Management

\begin{tabular}{|c|c|}
\hline Question: & $\begin{array}{l}\text { What are the biggest implementation challenges that a manufacturer would } \\
\text { face implementing MTConnect }{ }^{\circledR} \text { ? What are some of the resources to help? }\end{array}$ \\
\hline Answer: & $\begin{array}{l}\text { Partnering with people in the community to connect the machine tools is one } \\
\text { approach. An inexpensive power monitor is a 'low hanging fruit' example. } \\
\text { Legacy systems create the most challenges, because it requires the most "chasing } \\
\text { down" of information. Documentation of information from legacy equipment is } \\
\text { not always available. Age of equipment and inconsistency of controllers are } \\
\text { challenges. Data is unreliable from aging machines. Getting hardware to match } \\
\text { software is also sometimes a challenge. Even if you have access to data, it might } \\
\text { not flow at high enough rates. Sometimes one must lower data frequency to } \\
\text { lower the amount of noise that comes through. }\end{array}$ \\
\hline Question: & What are challenges as they relate to scaling? \\
\hline Answer: & $\begin{array}{l}\text { In a real-life example, a plant has } 75 \text { pieces of connected equipment requiring } 6 \\
\text { cell-based connection phases. Installation of all machines occurred at the same } \\
\text { time but each provides different data. You cannot replicate what you did for one } \\
\text { machine exactly with another. Wireless can often fail, so connections are still } \\
\text { hard-wired. Our shop also reconfigures cells, so rewiring everything is } \\
\text { challenging. One can segment machines into level of difficulty to install. The } \\
\text { next phase is hooking them up and performing data configuration. Managing } \\
\text { legacy equipment and wiring ultimately slows down the scaling process. Seldom } \\
\text { does maintenance data correspond with machine data. One solution is to } \\
\text { compare fault data to data under normal operating conditions to get a baseline. }\end{array}$ \\
\hline Question: & How do you balance the tradeoff between MTConnect® and OPC-UA. ? \\
\hline Answer: & $\begin{array}{l}\text { Object Linking and Embedding (OLE) for Process Control Unified Architecture } \\
\text { (OPC UA) provides an abstract model while MTConnect@ provides meaning } \\
\text { behind the model. MTConnect }{ }^{\circledR} \text { is more exact. More clearly, OPC UA is } \\
\text { focused on syntactic interoperability while MTConnect is focused on semantic } \\
\text { interoperability. Identifying the meaning of the data when performing analytics } \\
\text { with OPC-UA requires two models. Integration of OPC-UA and MTConnect }{ }^{\circledR} \\
\text { is possible. It is vitally important to understand your data and define what it } \\
\text { means, so it you can compare it on a consistent basis. }\end{array}$ \\
\hline Question: & What are current limitations of MTConnect ${ }^{\circledR}$ standards? \\
\hline Answer: & $\begin{array}{l}\text { Models are rolling into standards development, all of which are capability- } \\
\text { centric. Within streaming data, we can tell how a piece of equipment is doing on } \\
\text { a continuous basis. Having an upgrade pathway in terms of connectivity is now } \\
\text { vital. Note that MTConnect } ® \text { can also be used in additive manufacturing }\end{array}$ \\
\hline Question: & $\begin{array}{l}\text { From a technology provider standpoint, how much guidance on } \\
\text { implementation do customers need? }\end{array}$ \\
\hline Answer: & $\begin{array}{l}\text { A lot of educational, open source information is available; companies also sell } \\
\text { services. A lot of information is available but not in a central place. Informative } \\
\text { white papers under development will help communicate value of technology. In } \\
\text { one example, reports released Monday morning show parts produced; these } \\
\text { reports automatically show up in employees' inboxes. }\end{array}$ \\
\hline
\end{tabular}




\title{
7 Additional NIST Measurement Science Research Efforts
}

\subsection{Michael Brundage (Systems Integration Division, NIST)}

\author{
"Using Unstructured Work Order Data to Improve Maintenance Procedures in \\ Manufacturing" (May 9 Talk)
}

Dr. Brundage provided an overview of the current manufacturing maintenance paradigm. The challenge associated with the maintenance work order (MWO) data is the presence of natural language in the data (i.e., the data form is not easily computable). Jargon and tribal knowledge are important pieces of this data because people are writing it, making it difficult to identify issues in a consistent way. The approach in this NIST research effort included looking at MWO data, manually cleaning the data (this mitigates the issues that arise when different terminology is used to describe the same thing), and transforming the MWO data by tagging individual words for annotation (e.g., keywords).

An overview of the MWO data transformation process brought out key points. If the plant does not have meters, a manufacturer can look at times in between tagged words, such as "broken," to determine which devices need maintenance. Changing up labor requirements based upon tagged words is also possible using this approach. Tagging can also be visualized geospatially (e.g., which equipment in the plant has the word "leak" associated with it the most). In comparing words used in MWOs among manufacturers, overlap exists, but many domains have industry-specific terminology. The tagging tool is ready for pilot deployment; the next steps are to test it with industry and develop standard guidelines for cleaning the data.

Dr. Brundage's slide deck is included in Appendix D - NIST Presentations.

\subsection{Brian A. Weiss (Intelligent Systems Division, NIST)}

"NIST Research on Monitoring, Diagnostics, and Prognostics for Manufacturing Workcells" (May 9 Talk)

Dr. Weiss summarized the PHM-related efforts NIST has been working on in recent years, including past workshops, collaboration with universities and industry, and interactions with various technical organizations. Research includes 1) manufacturing process and equipment monitoring, 2) health and control management for robot workcells, and 3) machine tool linear axes diagnostics and prognostics. Each of these three research efforts is supported by physical testbeds that allow the physical instantiation of representative manufacturing use cases, the development of test methods, collection of reference datasets, and a platform to test software tools. The goal of the manufacturing process and equipment monitoring effort is to identify high-value data sources and the most appropriate opportunities to collect data to avoid the challenges of big data. The goal of the robotic workcell project is to develop the necessary measurement science to enable V\&V of PHM technologies within a manufacturing robot workcell, down to the robot, and sub-robot levels. The goal of the machine tool linear axes diagnostics and prognostics effort is to develop a sensor-based method to efficiently estimate the degradation of linear axes. 
A primary question that NIST and stakeholders must answer is whether there is a costeffective, methodical approach to guide manufacturers through the PHM design and deployment process without knowing all failure modes. Another key question is what equipment or processes can/should be monitored and how. Next steps include updating research efforts to reflect the changing needs of industry; further develop test methods and performance metrics; facilitate strategic collaborations with industry to pilot test methods and exchange feedback; and gain greater understanding of common configurations.

Dr. Weiss' slide deck is included in Appendix D - NIST Presentations.

\section{Standards and Best Practices}

\subsection{Emerging Research Efforts}

\subsubsection{Junmin Lee (Seoul National University)}

"Exercising Standardization of Prognostics and Health Management (PHM) for Manufacturing Industry" (May 9 Talk)

Mr. Lee provided an overview of PHM, its importance, and impacts on manufacturing operations. He proposed PHM to be the focus of a standard framework that determines industry productivity with respect to health features for specific systems types. Based on an industry survey, most South Korean manufacturers want to integrate PHM. A standard framework for users without PHM backgrounds is required to accomplish this goal. The methodology is applied in six respective steps: 1) identification of system types, 2) collection of system information, 3) acquisition of data, 4) extraction of health features, 5) diagnosis of faults, and 6) performance of a cost /benefit analysis. This framework can be enhanced by obtaining reference tables that help users choose PHM approaches suitable for each target system.

\subsection{Panel on Standardization and Best Practices}

\subsubsection{Andrew Hess (Hess PHM Group)}

"PHM - A Key Element across the Continuum of a Digital Enterprise" (May 10 Panel)

Mr. Hess discussed predictive analytics for assisting with asset management, using an aircraft carrier as an example. Predictive analytics are at the heart of aircraft carrier asset management, and can significantly influence manufacturing operations. Merging big data, small data, and nontraditional data is currently happening and will continue to happen in the future. Mr. Hess sees PHM as a set of capabilities, information products, and mixed technologies applied for the field, e.g., applying to standards such as SAE E-32 $2^{12}$. PHM can show the engine manufacturers that this is important. The standards development process can bring people together to start a dialogue. If you have a predictive element, you

${ }^{12}$ https://www.sae.org/works/committeeHome.do?comtID=TEAE32 
can change control schedules; this demonstrates an ability to shut down equipment at a given time if not needed.

During the life of the system, many failures occur during initial implementation and end of life (EOL) product stages. It is important to set an alarm threshold with an appropriate amount of lead-time. This enables manufacturers to determine where uncertainty levels reside. Good diagnostics can serve as an insurance policy. PHM implemented early in the lifecycle can be more expensive. A hybrid method between data-driven and physics-based approaches is best. However, there is a strong need to have tools to deal with vast amounts of data. Zero maintenance systems are the ultimate goal; getting close to this is a reasonable possibility.

\subsubsection{Ravi Rajamani (drR2)}

"The Role of Standards in Designing and Maintaining Complex Systems" (May 10 Panel)

Dr. Rajamani presented on the importance and usefulness of standards in large engineering design projects. Standards are critical and typically used judiciously. Large engineering projects, such as aerospace systems development, are dependent on these standards because of critical safety implications. A few examples of standards being critical for human survival include the Great Baltimore Fire of 1904, which spread due to lack of uniform threads on fire hydrants. Great interest exists in the aerospace industry to develop PHM standards. Current PHM systems in aircraft include engine vibration monitoring, extended engine operations, and engine health management (such as monitoring oil debris online rather than inspection to reduce costly and timely inspections and obtain "maintenance credits"). PHM standards are beneficial and needed for aerospace and manufacturing industries. Typical aviation maintenance practice consists of periodic inspections followed by repair for cause, unless the parts are life-limited, in which case they are replaced at end of life. With certified PHM systems in place, it is possible to reduce or eliminate periodic inspections and only conduct them based on indicators from the PHM system. The process of obtaining this "relief" from the authorities to lengthen inspection intervals or entirely eliminate them is known as obtaining "maintenance credits."

\subsubsection{Tom Fiske (Yokogawa)}

"ISA108 Intelligent Device Management" (May 10 Panel)

Dr. Fiske provided an overview of the International Society of Automation (ISA), and the resources they offer, such as standards, certification programs, education, and publications. One effort within ISA involves developing a standard centered around Intelligent Device Management (IDM) - ISA108. IDM is important because it promotes doing repair only where necessary and provides an environment to properly use automation devices while considering condition and risk management. Intelligent device standards are complex. Device lifecycles are much shorter than the life of the facility, so IDM standards need to take advantage of new technologies and features on an asset management level. Opportunities exist for supply chain standards development: it may be easier to work with a few devices when new equipment is available. A master database is useful to maintain consistency of data, but also to manage changing device classifications. You cannot always just replace a part; often there are upgrades to equipment because the old (i.e., original) 
equipment is no longer available. Asset management is also dependent on how important the component is to the entire system. The goal is to detect a problem at an appropriate time horizon, optimize the maintenance process, and select an appropriate strategy before the problem has too many adverse effects on the entire system. The ISA108 standards defining the IDM program's non-normative concepts and terminology now exist, but standards regarding condition management, alert management, and critical device availability requirements are still in development. A need exists to address significant barriers and issues in the IDM effort which is leading to the assembly of working groups for expediting IDM standard development.

\subsubsection{Tom Hedberg (NIST - Systems Integration Division)}

"Challenges in Standards for a Model-Based Enterprise" (May 10 Panel)

Dr. Hedberg presented on challenges in developing standards for coupling existing technologies with trusted systems, IoT, big data, and AI. Open architectures are needed to help to support "brownfield" (i.e., where new and legacy technologies are integrated together) realities of manufacturing, allow interconnectivity across decentralized systems, and enable closer manufacturing to the end user. It is not easy to integrate technologies and standards vertically from process to sense and monitor the manufacturing execution system (MES) and enterprise resource planning (ERP). Mr. Hedberg defines three classes of Standards: 1) Practice and Specification, 2) Data and Information, and 3) Modeling.

PHM does more than provide machine health measures; it converges design changes and manufacturing phases into an ecosystem that promotes health during the whole lifecycle. With manufacturing advancements and changing consumer expectations for product design and implementation speed, two important questions arise. One, how do we match product needs to process capabilities? Second, within a PHM context, how do we sacrifice what exists today to ensure availability of systems tomorrow? Developing and implementing standards is the answer because they normalize product and process definitions, as well as integrate product, process, and logistics viewpoints.

Dr. Hedberg's slide deck is included in Appendix D - NIST Presentations.

\subsubsection{Logen Johnson (SAE)}

"Best Practices in Developing PHM Standards: SAE International” (May 10 Panel)

Mr. Johnson presented on SAE International's practices of developing PHM standards, as well as case studies for standards implementation. This included an overview of SAE International, their portfolio, and standards implementation in the aerospace industry. A few case studies on PHM standards in the aerospace industry illustrate that maintaining standards means ensuring they are flexible enough to match industry progress. Success story case studies, such as SAE E-32 for engine vibration monitoring systems and SAE ARP5987 $7^{13}$ for maintenance credits using engine health management (EHM), are good examples.

\footnotetext{
${ }^{13}$ https://www.sae.org/standards/content/arp5987/
} 
A few challenges inhibit developing and implementing standards. It is extremely difficult to have standards that keep pace with technology innovation (a common theme). Multiple iterations and revisions become necessary. A standard way to talk about health-ready components currently does not exist, but SAE is working on developing that. SDOs often do not work as closely together as they should, which can cause inconsistencies in reporting application requirements in their respective applications. Sharing information and harmonizing terms is crucial for future PHM standards. Moving beyond aerospace and automotive standards that SAE has championed, SAE has created G-23 - a Manufacturing Management Committee. This committee is focused on addressing manufacturing issues.

\subsubsection{Donnie Alonzo (ASME)}

"The American Society of Mechanical Engineers: ASME Manufacturing Standards Overview" (May 10 Panel)

Mr. Alonzo provided an overview of the ASME mission statement and standards developed to improve public safety and provide uniform product consistency. ASME standards rely on knowledgeable experts in industry, academia, and government, and aim to reach an international audience, when possible and appropriate. A few challenges impede determining new standards. ASME standards do not receive funding from industry or government; are voluntary unless adopted into law by a regulatory authority; and may become quickly outdated depending upon the target application. Monitoring, prognostics, and diagnostics for manufacturing (i.e., process PHM), as well as Model-based Enterprise (MBE), are still being developed into standards by ASME. Monitoring, prognostics, and diagnostics are of interest to ASME. Two workshops provided input on this topic: one held in June 2017, in conjunction with the Manufacturing Science and Engineering Conference (MSEC), and another workshop held in October 2017 at the PHM Society Conference. A few important next steps proposed include forming a charter to identify a work breakdown structure to address PHM priority areas; and establishing standards and guidelines to provide information on terminology, design, implementation, verification, and validation of PHM technologies.

Panelists responded to audience questions during a Q\&A session following the panel on standards and best practices. Table 10 summarizes the results of those discussions. 
Table 10 - Q\&A - Standards and Best Practices

\begin{tabular}{|c|c|}
\hline Question: & $\begin{array}{l}\text { What are the main goals of each of these SDOs? If many work on similar items, } \\
\text { who has the final word? }\end{array}$ \\
\hline Answer: & $\begin{array}{l}\text { The answer is industry; these are all voluntary standards. The standards are only as good } \\
\text { as the people who help develop them. It is cheaper for companies to comply with } \\
\text { standards rather than develop their own. Sometimes standards organizations disagree } \\
\text { and branch off to start their own version, but they must come together in the end. } \\
\text { "Recommended practice" documents (not standards) might be most useful. They } \\
\text { describe the state of the art and best practices. SAE produces different levels of } \\
\text { standards, purely for information. Maybe one SDO role could be to merge standards } \\
\text { developed by other SDOs. While having parallel SDOs working on similar standards } \\
\text { may seem duplicative, it usually ends in a better product. ANSI }{ }^{14} \text { represents America's } \\
\text { voice in the standards community and prefers the ultimate standards consumers (e.g., } \\
\text { industry) to have a strong influence within this community. For additive manufacturing, } \\
\text { ANSI stepped in and facilitated collaboration when many SDOs were developing } \\
\text { standards. } \\
\text { The PHM Society has a standards committee to look across different standards groups } \\
\text { to see what is going on across SDOs to look for gaps and distribute work to fill gaps. } \\
\text { Many ASME members work with other SDOs, and stay abreast of other SDO efforts. } \\
\text { Standards are just a small part of what these organizations do; they also educate end } \\
\text { users and provide training certificate programs. }\end{array}$ \\
\hline Ques & $\begin{array}{l}\text { Can we model at once for an entire enterprise? How is it you were inspired to take } \\
\text { this very high-level, enterprise-wide approach (model-based enterprise)? }\end{array}$ \\
\hline Answer: & $\begin{array}{l}\text { I [Dr. Hedberg] worked in aerospace sector for } 10 \text { years trying to deploy digital } \\
\text { technologies across all organizations. There are different countries involved, people with } \\
\text { different roles and responsibilities, and other challenges. At the end of day, the same } \\
\text { issues need addressing. We do not have technologies and standards for doing this across } \\
\text { entire industrial enterprises; a paradigm shift needs to happen. Regulatory reform policy, } \\
\text { as well as technology and psychology, will change perceptions. }\end{array}$ \\
\hline Question: & $\begin{array}{l}\text { In some cases, best practices are better than standards. How do you choose between } \\
\text { a standard, guideline, or best practice to address an issue? }\end{array}$ \\
\hline Answer: & $\begin{array}{l}\text { This determination typically comes from the people attending the committee meeting. } \\
\text { The Internet has been a great tool for hands-on information. One of the biggest barriers } \\
\text { is how to keep up with current progress on the standard and related decision-making, as } \\
\text { well as what is available in draft form. ANSI and other accreditation boards can help } \\
\text { overcome this challenge. Another challenge during the development process is providing } \\
\text { materials to SDOs. These become the property of the SDO (copyrighted) so the material } \\
\text { cannot be used without permission. This is sometimes a barrier to member input to the } \\
\text { working groups. }\end{array}$ \\
\hline
\end{tabular}

${ }^{14}$ https://www.ansi.org/ 
Table 10 (cont'd) - Q\&A - Standards and Best Practices (cont'd)

\begin{tabular}{|l|l|}
\hline Question: & Where is the most disagreement in the PHM standards development world? \\
\hline & $\begin{array}{l}\text { From the aerospace perspective, eliminating inspection by humans through } \\
\text { replacing some people with an Engine Health Management system (for vibrations) } \\
\text { caused significant disagreement. This is controversial and creates a major barrier. } \\
\text { Many think having humans in the loop is safest. For specification requirements, we } \\
\text { could only use performance-based information, not standards. If good standards are } \\
\text { not possible, maybe a standard stating what not to do is; this reduces repetition of } \\
\text { the same mistake. } \\
\text { Answer: } \\
\text { People tend to want to stay with what they are most familiar, and perspectives need } \\
\text { to change to be more open. While we may not reach 100\% of the desired outcomes } \\
\text { for standards, compromise is possible (but a major challenge). }\end{array}$ \\
$\begin{array}{l}\text { Large companies come in and try to influence standards, then branch off and work } \\
\text { with others to develop the standard they want. People in standards committees } \\
\text { come from different backgrounds and multiple disciplines, so each have unique } \\
\text { biases. It is a challenge to mitigate the effects of siloed viewpoints on other } \\
\text { disciplines. }\end{array}$ \\
\hline $\begin{array}{l}\text { Would it make sense for SDOs to get together and develop one set of universal } \\
\text { guidelines rather than have separate ones? }\end{array}$ \\
$\begin{array}{l}\text { SDOs are careful about how to work with one another. They engage where } \\
\text { activities do not involve copyright infringements, and within their capacity. Co- } \\
\text { publishing creates its own set of difficulties between SDOs. The first step for an } \\
\text { SDO developing their own standards is to conduct research on what is available; } \\
\text { the ultimate objective is for everyone to use one set of standards. SDOs also } \\
\text { generate revenues; and copyright ownership is the source of revenue. }\end{array}$ \\
$\begin{array}{l}\text { Scope creep often occurs where standards can start to blend. Standards } \\
\text { development needs a universal system approach, including identifying } \\
\text { stakeholders, understanding needs, and conducting verification and validation to } \\
\text { ensure stakeholder needs are met. }\end{array}$ \\
\hline Answer:
\end{tabular}

\section{International Perspectives on PHM}

\subsection{Byeng Youn (Seoul National University)}

"PHM Frontiers in Korean manufacturing - Success Episodes and Issues" (May 10 Talk)

Dr. Youn presented on PHM in Korean manufacturing from both a theoretical and a practical viewpoint. The South Korean government launched the Korea Smart Factory Foundation (KOSF) in 2014, which mainly targets small and medium factories. Smart factories increased through this program, which is key for boosting the Korean economy and manufacturing competitiveness. Recent accomplishments in this program included being able to handle balancing physical data with AI and winning the Global PHM Data Challenges competition five out of six years over various industrial sectors. There is a need to standardize four different PHM approaches: data-driven, data plus physics-based (hybrid), physics-based, and rule-based; rule-based approaches should only be applied if the previous three are infeasible. Future challenges include poor data quality, lack of 
labeled data, lack of resources, and cyber security and data ownership. PHM is a common discipline for industrial information prognoses, including machine health and product health. Four different case studies provided insight.

- Case 1: Industrial Robot - Downtime costs \$200,000; market size is \$2.64 billion. This project uses a physics-based approach, then a database approach using vibration signal or motor current for PHM. A spot-welding process provided validation. The object is to acquire data with constant rpm; minimizing the amount of data required is a goal. Minimizing data and maintaining ability to detect faults is important.

- Case 2: Industrial Bearing Rolling-Element Bearing - Bearings used in wind turbine motors, pumps, and similar equipment are the focus. Software predicted the faults and remaining life of the bearing. This intelligence was presented through a dashboard of live bearing monitoring metrics.

- Case 3: Overhead Hoist Transport $(O H T)$ - The focus is to detect faults using PHM.

- Case 4: Deep Learning - The focus is to collect gap sensors analysis output in a fault log and determine probability of failure.

Following his talk, Dr. Youn fielded questions from the audience (summarized in Table 11).

Table 11 - Q\&A - PHM Frontiers in Korean Manufacturing - Success Episodes and Issues

\begin{tabular}{|l|l|}
\hline Question: & $\begin{array}{l}\text { For the robot case study, you measured current and vibration. Did you } \\
\text { measure vibration with additional sensors? }\end{array}$ \\
\hline Answer: & $\begin{array}{l}\text { Yes, vibration measurements require additional sensors. The output obtained } \\
\text { was more sensitive than that from the current measurement. Performing current } \\
\text { measurements does not require additional sensors. }\end{array}$ \\
\hline
\end{tabular}

\subsection{Minchul Lee (Pohang University of Science and Technology)}

"Data-Driven Prognostics for an Assembly Machine for Automatic Transmissions" (May 10 Talk)

NOTE: Minchul Lee presented in place of Dr. Hyunbo Cho due to an unforeseen schedule change.

Mr. Lee presented the results of a government-funded data-driven prognostics project for an automatic assembly line for a transmission drum. This is a core part of the automatic transmission factory because it is a continuous manufacturing line. As a result, if one motion stops, the whole line stops. The OEE of the company is $85 \%$, which means that the overall production efficiency loss is $15 \%$. The majority of the loss is due to equipment failure (49\%), then idle and minor stops (28\%), which were allowed within the scope of the project. The goal of the project was on reducing minor stops (e.g., screw jams, misaligned sensors, disconnection of harness, etc.). The team developed a data-driven model to help predict what causes minor stops. The model clustering method separated out 
minor stops from stops with unknown reasons using the following methodology: 1) Clustering, 2) Fault Tree Analysis, 3) Fixed Variables, and 4) Repeat Cluster. Data reliability was an issue during the project, which is a lesson learned for future studies.

\subsection{Hyunseok Oh (Gwangju Institute of Science and Technology)}

"Korean Efforts towards PHM in Semiconductor and Automotive Manufacturing" (May 10 Talk)

Dr. Oh presented on PHM efforts for semiconductor and automotive manufacturing in Korea. The semiconductor and automotive sectors are Korea's two largest major exports at $20 \%$ and $14 \%$, respectively. The EBITDA (earnings before interest, taxes, depreciation, and amortization) margin in these sectors are $50 \%$ and $11 \%$ for the semiconductor and automotive industry. For the sake of comparison, the EBITDA margin for Amazon and Apple is $3 \%$ and $20 \%$, respectively. One negative impact on the margin of these key sectors - recent power outages in Korea have led to significant financial losses due to unscheduled downtime (e.g., a 30 min power outage in semiconductor manufacturing led to $\$ 46$ million loss); enhanced PHM capabilities could have mitigated these power outages.

Two PHM case studies illustrate recent trends. Several small semiconductor companies are no longer in business and bigger ones took over between 2009 and 2017. PHM can contribute to their competitiveness and improve yield. For example, vibration analysis on vacuum pumps can help to understand health conditions of vacuum pumps and determine optimal maintenance schedules for semiconductor facilities. Another example is to monitor electric waveforms of inverter systems in automotive manufacturing lines. These efforts are expected to reduce maintenance costs and prevent potential losses of semiconductor equipment. Furthermore, in production lines such as steelworks, deep learning can help to resolve scalability problems of PHM techniques by autonomously extracting features from data collected from heterogeneous systems. This can relieve the burden of customizing a particular PHM technique to numerous systems with different scales and identical working principles.

\subsection{Chan Hee Park (Seoul National University)}

"Fault Detection for an Overhead Hoist Transport Vehicle" (May 9)

Mr. Park presented emerging research efforts for fault detection in overhead hoist transportation systems. The drive for this research is the desire to detect failure without additional sensors since sensor installation can be difficult. The control signals such as speed and torque can be acquired readily because they are originally accessed for control purposes. Abnormal indicators cause unexpected control changes. The health index used for the overhead hoist transportation system was a variance of filtered signal (VFS) for each type of failure. The VFS increases result in belt and bolt loosening and motor degradation. These controls also assisted in decreases in gearbox oil leakage, so are important to overall performance. 


\title{
10 Visualization Tools for PHM
}

\subsection{Jeremy Marvel (Intelligent Systems Division, NIST)}

\author{
"Visualization Tools for PHM: Metrics of Effective HMI" (May 10 Panel)
}

Dr. Marvel presented on PHM visualization tools and their respective metrics for an effective human machine interface (HMI). Three Mile Island is used as an example to highlight the importance of the HMI. Pump failure led to reactor cooling failure, so plant operators shut down the reactor without realizing heat was still building up. Built-up heat caused an increase in pressure, which subsequently opened the relief valve. When pressure was reduced back to normal levels, the valve should have closed, but did not. These misinformed decisions caused reactor number two to experience partial meltdown. The lesson learned is to make sure your interface is meaningful so human personnel could easily understand the state of the system and take the appropriate actions at the right time horizons.

Interfaces for robotics have changed over the years to reduce complexity. The machinery or purpose dictates what the interface will look like, as well as the input information and feedback the interface will provide. Two examples where this is happening today:

- Consumer Goods: Products are becoming more and more human-centric where human needs are considered

- Industrial Goods: Not as human-centric, but slowly trending in that direction.

The metrics for these interfaces include software quality (and standards), interface interaction (and standards), and user response. There are metrics and standards for interfaces and software yet no one uses them. One of the most important aspects for interfaces and software is to improve the human robot interaction (HRI).

Dr. Marvel's slide deck is included in Appendix D - NIST Presentations.

\subsection{Sinan Bank (Siemens Corporation)}

“The Use of Mixed Reality for Manufacturing” (May 10 Panel)

Mr. Bank presented on the Siemens Corporation's involvement in R\&D of digitalization technologies for manufacturing applications and their overall R\&D portfolio, which has a $\$ 5.2$ billion budget. The idea of digitally-enhanced electrification and automation has a holistic perspective. A few examples of this are making automated decisions, trying to create robots that build something new that has yet to exist, and expanding the twin in digital domain. Mr. Bank goes on to articulate the difference between virtual, augmented, and mixed reality is:

- Virtual reality (VR) - there is a stimulus in which you react, without physical elements

- Augmented reality (AR) - you overlay images onto a visualization of the physical world

- Mixed reality (MxR) - a form of AR that blends elements of VR and AR together. 
Siemens has a system where people can interface with experts remotely if issues arise (e.g., remotely diagnosing issues with plants in the Middle East when an expert cannot travel to the plant, directly). Oculus Rift ${ }^{\circledR}$ and Google Glass ${ }^{\circledR}$ started the success of virtual and augmented realities in 2013. Training and guidance will significantly promote VR in manufacturing (e.g., digital twin of factory layout, person inspecting a car with VR glasses), which can eliminate the requirement of being on the plant floor. Future applications involve scanning the plant and producing a digital twin without plant plans. Siemens' goal is to make VR more immersive, so you can really see everything in the manufacturing plant, including any device issues.

A Q\&A session followed the panel on visualization tools. Table 112 summarizes the main discussions points.

Table 112 - Q\&A - Visualization Tools for PHM

\begin{tabular}{|c|c|}
\hline Question: & Do cultural biases cause crashes? \\
\hline Answer: & $\begin{array}{l}\text { Yes, the way something appears can lead to cultural implications. In many } \\
\text { instances, developers do not consider cultural biases. }\end{array}$ \\
\hline Question: & Are you also interested in visualizing at the part level? \\
\hline Answer: & $\begin{array}{l}\text { Disconnects exist between the person and the part, and we want to interact with } \\
\text { the part. The object is to overlay data without moving anything. }\end{array}$ \\
\hline Question: & Is there a roadmap for prognostics with humans? \\
\hline Answer: & $\begin{array}{l}\text { Yes, there is a separate user interface; John Deere, Ford, and Boeing are now } \\
\text { using it with motion capture devices. The purpose is to see ergonomically what } \\
\text { is happening. Human kinematic analysis is not complete right now. We should } \\
\text { visualize PHM for the human part of an operation, integrating the human to the } \\
\text { manufacturing process. Our primary focus is ergonomics. }\end{array}$ \\
\hline Question: & $\begin{array}{l}\text { Industry is not adopting some standards. Where do you see standards in the } \\
\text { future for PHM? }\end{array}$ \\
\hline Answer: & $\begin{array}{l}\text { The Three Mile Island incident accelerated PHM standards development. There } \\
\text { may be issues of reliability (e.g., for medical applications), and certain industries } \\
\text { will need to have standards. The way that manufacturing domains interact today } \\
\text { is disparate, with a lack of communication in some cases on standards. We } \\
\text { currently have some standards in place to come up with feedback for process } \\
\text { control. }\end{array}$ \\
\hline Question: & Are you using virtual reality during the design phase for inspect-ability? \\
\hline Answer: & $\begin{array}{l}\text { Virtual reality for design is in the } R \& D \text { phase. It is in the roadmap but not } \\
\text { deployed. Collaboration is very important. We are members of a cloud-based } \\
\text { coding platform to facilitate collaboration. }\end{array}$ \\
\hline Question: & Is there user-conformable HMI? Users have different opinions. \\
\hline Answer: & $\begin{array}{l}\text { Yes, a key factor for usability is to have a level of customizability. Just like a } \\
\text { smartphone, you can reorganize the layout of buttons and apps. Having an } \\
\text { operator customize the HMI is important. }\end{array}$ \\
\hline
\end{tabular}


Table 122 (cont'd) - Q\&A - Visualization Tools for PHM

\begin{tabular}{|l|l|}
\hline Question: & Are we measuring the performance of augmented reality safety training? \\
\hline Answer: & $\begin{array}{l}\text { Some research is underway on transfer learning by using virtual reality. One } \\
\text { robot learns and can transfer that knowledge to the other robots through the } \\
\text { cloud. An embedded semantic system then converts that task to another robot. } \\
\text { For the human pieces, tribal knowledge is a big step. Digitizing a human action } \\
\text { would be a major accomplishment. Today, virtual reality is in use for safety, risk } \\
\text { assessments, and identifying new risks. }\end{array}$ \\
\hline Question: & $\begin{array}{l}\text { The HMI and Human Computer Interaction (HCI) community have similar } \\
\text { messages but go to different conferences. How do we increase interaction } \\
\text { between these groups? }\end{array}$ \\
\hline Answer: & $\begin{array}{l}\text { The HCI community is encroaching on other territories. Computer science } \\
\text { people drive tablets technologies. The manufacturing HCI should talk with those } \\
\text { creating videogames. }\end{array}$ \\
\hline
\end{tabular}

\title{
11 Planning for the Future - Building and Leveraging Artificial Intelligence
}

\subsection{Michael Garris (Information Technology Laboratory, NIST)}

\author{
“Demystifying Today’s Artificial Intelligence” (May 10)
}

Mr. Garris presented on the state of AI, and the national strategies and opportunities for AI at NIST. AI is currently present within many technologies including unmanned systems, robotics, and speech recognition. One accepted definition of AI is a system that thinks and acts like humans; and thinks and acts rationally. AI can encompass multiple problem space categories including knowledge representation, perception, logical reasoning, planning/ navigation, and prediction. AI is not a new technology; it is facing a resurgence as supporting technologies (e.g., sensors, database solutions, computers, networking) are becoming more capable and accessible and is a viable tool to enhance previously-untapped domains (e.g., manufacturing). A conceptualization of AI encompasses Machine Learning (ML). In turn, ML encompasses Deep Neural Networks (DNNs) (see Figure 5).

As AI evolves, the concepts of Narrow AI and General AI have emerged to distinguish the capabilities and limitations of today's AI technologies, from that of the future. Narrow AI can be characterized as application specific/task limited, leveraging fixed domain models provided by expert programmers, and limited in that knowledge does not transfer to other domains or tasks. AI is evolving to what is termed as General AI and is characterized as capable of performing general (human) intelligent action(s), self-learning and reasoning with its operating environment, learning from a few examples and/or unstructured data and

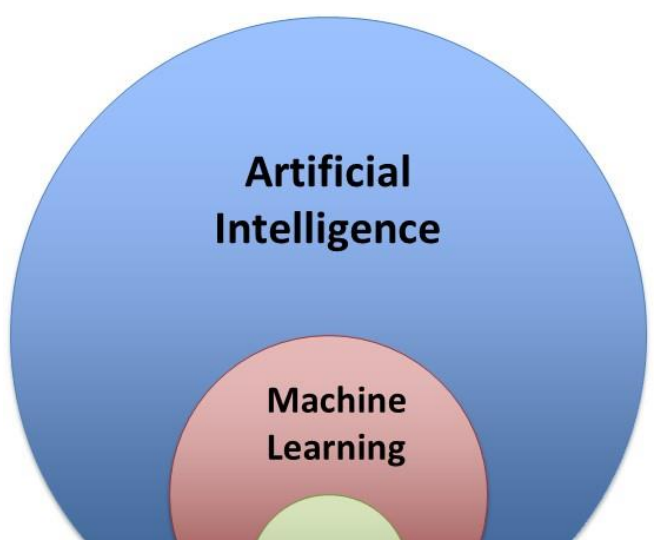

Figure 5: AI Conceptualization 
transferring knowledge to new domains and tasks. This evolution is being made possible through the availability of big data, improved ML algorithms, greater computing power, and mobile connectivity of sensors, user interfaces, and other enabling hardware.

ML/AI are being used in multiple functions. This includes classification, function approximation, prediction, control, simulation, and anomaly detection. Specific to manufacturing, ML/AI has application to advanced data analytics (e.g., to promote predictive maintenance technologies, optimize both component and end-to-end systems) and human assistive technologies (e.g., automated inspection and quality control, collaborative robotics). As ML/AI technologies are data-driven, their development requires a fundamentally different workflow from traditional software engineering. The AI community has developed a generalized five-step workflow: 1) capturing data, 2) cleaning, preparing, and manipulating the data, 3) training the model(s), 4) testing the model with different data, and 5) improving the model through iteration.

There are significant challenges to AI and machine learning. These probabilistic technologies are not without error. With data-driven aspects, a challenge is the vulnerability of unwanted behaviors that come out of the training. Extreme dimensionality is another challenge; this is making decisions opaque with little explanation. Today's AI is capable of delivering systems that solve amazing problems, but the ability to interpret how or why they work is limited. AI will not solve every problem; however, it can provide significant contributions to a wide range of problems. AI also has lifecycle costs; the longer a machine learning solution is deployed within an operation, the more expensive it is to maintain.

Mr. Garris' slide deck is included in Appendix D - NIST Presentations. Table 13 summarizes the questions and answers following this presentation.

Table 13 - Q\&A - Demystifying today's Artificial Intelligence

\begin{tabular}{|l|l|}
\hline Question: & Is AI affected by factors at each components' station? \\
\hline Answer: & $\begin{array}{l}\text { Manufacturing is always a cascade of interactive processes and now connectivity } \\
\text { via IoT. The end-to-end system has a final uncertainty but all components } \\
\text { contribute [to the uncertainty]. NIST has worked to characterize physical devices } \\
\text { for years and now AI can aid in this characterization process. It is cheaper but } \\
\text { has some uncertainty. How do we characterize the tradeoff between cost and } \\
\text { uncertainty? Maybe the answer is adding machine learning and data analytics. }\end{array}$ \\
\hline Question: & $\begin{array}{l}\text { When it comes to AI, do we look at safety aspects first then build out a } \\
\text { system or the other way around? For example, surgery would require more } \\
\text { safety than another discipline. }\end{array}$ \\
\hline Answer: & $\begin{array}{l}\text { The order would be determined on a case-by-case basis. There are advantages } \\
\text { for both approaches. Some international parties are trying to issue blanket } \\
\text { policies to address this, while the United States takes a free-market approach. }\end{array}$ \\
\hline
\end{tabular}


Table 143 (cont'd) - Q\&A - Demystifying today's Artificial Intelligence

\begin{tabular}{|l|l|}
\hline Question: & $\begin{array}{l}\text { One big challenge of AI in the 90s was the "knowledge acquisition } \\
\text { bottleneck" and it is still an issue today. We need common dictionaries } \\
\text { within industry. What is the low hanging fruit for AI and how do we } \\
\text { integrate it in manufacturing? }\end{array}$ \\
\hline Answer: & $\begin{array}{l}\text { It is great if a community can agree upon terminology. If there is legacy data to } \\
\text { filter through, that becomes a challenge. A lot of attention is going into all kinds } \\
\text { of AI; deep learning is an example. Deep learning is an analog of the visual } \\
\text { perception system. Northwestern University was doing symbolic reasoning in } \\
\text { 80s. They are also benefitting now from research dollars attached to AI. } \\
\text { However, there is no further structuring of knowledge or interaction between } \\
\text { memories. The hope is that these will be brought together in a more powerful } \\
\text { way. }\end{array}$ \\
\hline
\end{tabular}

\subsection{Jay Lee (University of Cincinnati, Center for Intelligent Maintenance Systems)}

"Industry AI - A System Perspective in Machine Learning for Smart Manufacturing and Maintenance" (May 10)

Dr. Lee presented three important messages associated with industrial AI: 1) maintenance is the last resort of manufacturing; 2) industrial big data is more than just maintenance; and 3 ) modeling of invisible relationships is critical. One problem is that people maintain logic for themselves, so there is a need to create knowledge by documenting and creating relationships. Another specific challenge is if you use data without the background, it is not possible to draw context from it, thereby rendering the data useless. Harvesting jet engine data provides an interesting example regarding the application of industrial AI [to the data]. Jet engine data at a single altitude, alone, is insufficient; data is needed at different altitudes based upon air density. Therefore, the data harvesting process was inadequate because data at a single altitude is insufficient.

AI is a cognitive science and Industrial AI is a systematic discipline. As a systematic discipline, the applied Industrial AI process should be repeatable and verifiable. One algorithm does not apply universally to multiple problems; this indicates the need to have a full spectrum of learning methods (e.g., similarity-based, peer-to-peer-based). One example considers a power surge in a Toyota plant; the resultant surge pattern was modeled and classified using Industrial AI. The plant has not had a surge since the application of Industrial AI. AI should not be simply about trial and error. Industrial AI should build upon previous success stories, where the ability to model the invisible is the ultimate goal.

Dr. Lee fielded questions after his talk; Table 14 summarizes the result of this discussion. 
Table 154 - Q\&A - A System Perspective in Machine Learning for Smart Manufacturing and Maintenance

\begin{tabular}{|c|c|}
\hline Question: & $\begin{array}{l}\text { A key component for } \mathrm{AI} \text { is accumulation of knowledge. What is your } \\
\text { view for systematically organizing and reusing this knowledge? }\end{array}$ \\
\hline Answer: & $\begin{array}{l}\text { Categorization of knowledge is essential. Some information is extracted } \\
\text { from cause and effect relationship while other information is probabilistic in } \\
\text { nature. This needs to turn into judgment. The knowledge needs to be } \\
\text { digitized using numbers, not simple "yes" and "no." Understanding } \\
\text { relationships is more important than simply adding more sensors. Once you } \\
\text { know the relationship, remove some sensors and only keep the ones needed. } \\
\text { For example, AI can help to understand the relationship between DNA and } \\
\text { diseases. }\end{array}$ \\
\hline Question: & $\begin{array}{l}\text { Can you speak to the relationship of using filtered data vs. raw data for } \\
\text { predictive analytics? }\end{array}$ \\
\hline Answer: & $\begin{array}{l}\text { AI all comes down to how fast we can make a determination. AI researchers } \\
\text { should relentlessly strive to improve performance and speed. A need exists } \\
\text { to explore additional means by expanding the use of data. Once you have } \\
\text { the data, eliminate what is unnecessary. }\end{array}$ \\
\hline Question: & There is information in raw data, but we lack tools to analyze all of it. \\
\hline Answer: & $\begin{array}{l}\text { Having and understanding background information helps to develop a } \\
\text { learning system for relevance and context, and couple that with data is } \\
\text { necessary. }\end{array}$ \\
\hline Question: & What TRL level is the focus of the IMS Center? \\
\hline Answer: & TRL 3-7. Fundamentally-relevant but not pure research is desirable. \\
\hline Question: & $\begin{array}{l}\text { Advanced AI systems are fragile and depend on initial data used to } \\
\text { build them. Validating technology is expensive and difficult. How do } \\
\text { you deal with this problem? }\end{array}$ \\
\hline Answer: & $\begin{array}{l}\text { A sound baseline data set that can do "multi-criteria" is essential. We can } \\
\text { layer more data on top of this baseline. We can also learn from competing } \\
\text { algorithms to see if they have similar performance, this will improve } \\
\text { machine learning. }\end{array}$ \\
\hline Question: & Can you comment on the high cost of maintaining AI systems? \\
\hline Answer: & $\begin{array}{l}\text { Skills are expensive in this field. Developers did not have the energy to } \\
\text { improve old algorithms; instead they built new ones. Additionally, when a } \\
\text { problem becomes too complicated and poorly understood, it is difficult to } \\
\text { determine which algorithm to use. Big problems do not reach solutions by } \\
\text { using a trial and error approach. When someone leaves a company, } \\
\text { knowledge goes with them. We need a legacy benchmark system. We should } \\
\text { develop } 20 \text { to } 30 \text { working algorithms and build from that for different } \\
\text { applications. }\end{array}$ \\
\hline Question: & $\begin{array}{l}\text { To develop engineering products, you define necessary/desired features. } \\
\text { How do you assess if a feature is a "must," a "could," or a "should?" }\end{array}$ \\
\hline Answer: & $\begin{array}{l}\text { Take the methods from success stories, repeat them and apply them to a new } \\
\text { model. Feature selection may be different based on the problem, so it will } \\
\text { not be standardized (the underlying method can be). Predictive analytical } \\
\text { tools become critical to apply to industrial problems. Time series data is not } \\
\text { enough. }\end{array}$ \\
\hline
\end{tabular}


Table 14 (cont'd) - Q\&A - A System Perspective in Machine Learning for Smart Manufacturing and Maintenance

\begin{tabular}{|l|l|}
\hline Question: & What is the biggest opportunity for standards in this field? \\
\hline Answer: & $\begin{array}{l}\text { Currently, most standards are at the data level (e.g., connectivity, } \\
\text { transmission). We should look at methodology for evaluating the process, a } \\
\text { guideline or standard for the process, and a validation/certification standard. }\end{array}$ \\
\hline
\end{tabular}

\section{ASME Standards Meeting}

\subsection{Introduction to the Standards Meeting}

This portion of the Industry Forum focused on ASME's effort to establish a committee to address the needs of the manufacturing community in areas of monitoring, diagnostics, and prognostics. The objective of this effort is to bring the manufacturing PHM community together to provide input into the development of both the committee and the subsequent guidelines and standards that would be developed within this committee. Previous workshops helped to identify priority topics in PHM where standards or guidelines could facilitate the application of advanced PHM technology. An introductory presentation provided context as to the motivation for establishing this standards community and potential gap identified in previous workshops. During the course of the day, participants reviewed and revised a draft charter for a PHM standards working group; held discussions on the priorities from the June 2017 workshop $^{15}$ to identify which standards should be addressed first; and created action plans for future efforts.

\subsection{ASME Efforts in Monitoring, Diagnostics, and Prognostics - Donnie Alonzo and Steve Weinman}

"ASME - Setting the Standard for Safety and Global Relevance” (May 11)

Mr. Alonzo briefly presented ASME's mission and the importance of standards. Engineering standards improve public safety, allow for uniform consistency of products, and help ensure fair and free commerce and trade. Standards have a wide impact on both the engineering community that uses them and the public, at large. Monitoring, Diagnostics, and Prognostics (commonly referred to as prognostics and health management $(\mathrm{PHM}))$ is one of several focus areas ASME is undertaking related to manufacturing ${ }^{16}$.

ASME held two workshops to discuss the scope of standards development for PHM. The first workshop, held in June 2017, was co-located with the ASME Manufacturing Science and Engineering Conference (MSEC); the second took place in October 2017 at the PHM Society Conference. The six priority topics originally identified at the June 2017 workshop became the focus of the discussions at this Industry Forum. Likewise, the draft charter for the standards working group was also discussed at this meeting.

15 Summary Report on a Workshop on Advanced Monitoring, Diagnostics, and Prognostics for Manufacturing Operations. November 2017. https://nvlpubs.nist.gov/nistpubs/ams/NIST.AMS.100-13.pdf

${ }^{16}$ https://www.asme.org/about-asme/standards 


\subsection{Draft Charter}

A collaborative discussion aided in defining a charter for the PHM Standards effort ${ }^{17}$. The session began by presenting a draft charter for comment. After real-time feedback from the audience, the revised charter reads as follows:

Develop guidelines that advance the design and implementation of monitoring, diagnostic, and prognostic capabilities, along with ways of verifying and validating their performance, to enhance adaptive maintenance and operational control strategies within manufacturing.

Approximately one month after the Industry Forum, this charter was approved by the ASME Board on Standardization and Testing in concert with the formation of this subcommittee $^{18}$ (subcommittee on Monitoring, Diagnostics, and Prognostics) under the Manufacturing and Advanced Manufacturing (MAM)Standards Committee.

\subsection{Priority Focus Areas}

Participants reviewed six important topics related to PHM based on action items identified at the June 2017 workshop at ASME MSEC held at the University of Southern California (USC). An seventh suggested topic "Guideline to Determine Where to Perform PHM" was added to the list. A voting process was then used to develop priorities among the seven topics. Table 15 presents the results of the prioritization exercise. Participants also completed action plan worksheets for selected priority topics (Figures 5-7). The group developed an action plan for Topic 7 due to its importance. Descriptions of the seven topics are as follows:

\section{TOPIC AREA 1: Standardized Terminology for PHM Guideline}

Preliminary Idea: Guideline containing a list of defined terms relevant and important to monitoring, diagnostic, and prognostic capabilities and technologies within manufacturing. This guideline could contain a relational map to correlate relevant manufacturing and PHM terms so the larger manufacturing community can easily communicate with one another. The terminology can be in a hierarchal structure. Basic terms could initially help to setup basic concepts. Wider and/or deeper terms separated into different categories and sub-categories would provide greater context.

TOPIC AREA 2: Guideline to Determine What Health Data to Capture and Collection Strategies to Employ

Preliminary Idea: Guideline that aids the manufacturing community in determining what health data to capture, including what collection strategies to employ to obtain this health data. The development and assessment of cost and risk evaluation matrix(ces) could be highly beneficial in determining this data. Figure 6 describes the Action Plan developed for this topic. It is important to note that this Action Plan also combines some elements of Topic Area 3. As this Action Plan is further clarified, it is

\footnotetext{
${ }^{17}$ Approximately one month after the Industry Forum, ASME officially approved this effort to be a standards committee.

${ }^{18} \mathrm{https}: / /$ cstools.asme.org/csconnect/CommitteePages.cfm?Committee=102342234\& ga=2.175616354.1334159429.1541766577$\underline{1412488438.1523558086}$
} 
possible that these two topic areas will be combined or a separate Action Plan will be devised for Topic Area 3 that will take some of the content from this current Action Plan.

TOPIC AREA 3: Guideline to Determine What Sensors and Where They Should Be Deployed to Inform on Process/Equipment Health

Preliminary Idea: Guideline that would determine sensor function and the depth of expedition; a case study that would document living successes; and/or a technical book or document that would explain how people could go through the sensor identification and deployment process, and evaluate their operation and potential risk to determine if they should implement the PHM technology.

TOPIC AREA 4: Guideline for Implementing Sensor Data Fusion/Multi-Modal Data Fusion

Preliminary Idea: Guideline to support the fusion of multiple sensor data streams to generate a meaningful analysis of equipment or process health. The output should offer an understanding of the evolutionary path of multi-model data fusion. This would begin with plotting multiple data streams and looking for any anomalies, notifying others of these anomalies and continuing the process to estimate, simulate, and diagnose.

TOPIC AREA 5: Guideline to Determine Where and When PHM Capabilities should be added/integrated

Preliminary Idea: Guideline for the manufacturing community to determine where (e.g., equipment vs. process level, system vs. subsystem vs. component level) and when to enable PHM to improve certain processes, mitigate fault conditions, etc., across the factory floor. The guideline is expected to document common operational use cases/ needs, define clear roles and responsibilities within an organization to effectively execute PHM functions, and enable the development of cost justifications for implementing PHM. Figure 5 describes the Action Plan developed for this topic.

\section{TOPIC AREA 6: Expand MTConnect / Data Communications}

Preliminary Idea: Develop a guideline to serve as a companion to the MTConnect standard to aid in standardization of communications of PHM data, especially as MTConnect is becoming more widely used within the manufacturing community. The guideline should address data communication standards within and external to equipment or workcell.

TOPIC AREA 7: Guideline to Determine Where to Perform PHM Data Analyses Preliminary Idea: Guideline for the manufacturing community to determine where (e.g., within the organization, external to the organization) PHM analyses should be performed. Manufacturers are not only facing multiple options in terms of who should be performing their data filtering and analyses, but also where this should be done (e.g., locally, at edge, in the cloud, ... and under whose control). This would also include guidelines for ownership and governance of the data. Figure 8: describes the Action plan developed for this topic. 
Table 15 - PHM Topic Prioritization

\begin{tabular}{|l|c|}
\hline Topics (Action Plans Available for Shaded Topics ) & 5 \\
\hline $\begin{array}{l}\text { Guideline to Determine Where and When PHM Capabilities should be } \\
\text { added/integrated (Topic 5) }\end{array}$ & 4 \\
\hline $\begin{array}{l}\text { Guideline to Determine What Health Data to Capture/What Collection } \\
\text { Strategies to Employ (Topic 2) }\end{array}$ & 3 \\
\hline Guideline to Determine Where to Perform Data PHM Analyses (Topic 7) & 3 \\
\hline $\begin{array}{l}\text { Guideline to Determine What Sensors and Where they should be Deployed to } \\
\text { Inform on Process/Equipment Health (Topic 3) }\end{array}$ & 2 \\
\hline Standardized Terminology for PHM (Topic 1) & 2 \\
\hline Expand MTConnect/Data Communications (Topic 6) & 0 \\
\hline $\begin{array}{l}\text { Guidance for Implementation of Sensor Data Fusion Analysis/Multi-Modal } \\
\text { Data Fusion (Topic 4) }\end{array}$ & \\
\hline
\end{tabular}

Figures 4-6 illustrate the Action Plans developed for the prioritized topics. Some content of these action plans is derived from some of the original discussion of the priority topic areas at the first workshop in ASME MSEC in 2017, while the bulk of the plans were generated during the group discussions of the priority areas at the Industry Forum. Each Action Plan summarizes the topic area, Action Plan steps, milestones and deliverables, and performance targets. It is important to note that two groups worked independently in developing the following Action Plans: one group developed the Action Plan documented in Figure 4 while another group developed the Action Plans shown in Figures 5 and 6. Although efforts were made to keep each group within the scope of their respective topic areas, there are a few overlapping details within each Action Plan. It is expected that these Action Plans will be further developed to become more distinct efforts. 
Figure 6: Priority Guideline Action Plan for Topic 2 - Guideline to Determine What Health Data to Capture and Collection Strategies to Employ

DESCRIPTION: The parameters that are observable and attainable within a process define the process and equipment health data. The health of a process is also relative to the context of the process. The purpose of this guideline is to aid the manufacturing community in determining what health data to capture; to determine what collection strategies to employ to obtain health data; and to address the purpose of collecting health data and level of specificity. First, identify major failure points; next steps are failure analysis, identifying mechanisms of failure and observable symptoms of the failure. This process helps identify what health data to collect followed by needed sensors to supply data.

\begin{tabular}{|c|c|c|c|}
\hline TIME & GUIDELINE A CTION PLAN StepS & $\begin{array}{l}\text { MILESTONES/KEY } \\
\text { DELIVERABLES }\end{array}$ & $\begin{array}{c}\text { PERFORMANCE } \\
\text { TARGETS }\end{array}$ \\
\hline 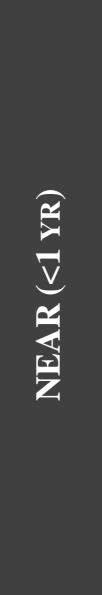 & $\begin{array}{l}\text { Examine the manufacturing process in its entirety } \\
\text { and identify high-risk failure points; some existing } \\
\text { standards may support this effort } \\
\text { - Reference existing standards with relevant } \\
\text { terminology and definitions (e.g., standards from } \\
\text { ASME, ASTM, International Organization for } \\
\text { Standardization (ISO), ISA, and SAE) } \\
\text { - Determine all potential data that needs to be } \\
\text { captured, regardless of sensor level and } \\
\text { availability; consider economics of collection } \\
\text { - Determine data priorities } \\
\text { - Determine necessary part and process qualities } \\
\text { - Collect health specifications, as built (from part } \\
\text { manufacturer, including configuration data), } \\
\text { actual/historical usage, projected/ intended usage } \\
\text { (see Notes) }\end{array}$ & $\begin{array}{l}\text { - Analysis procedure } \\
\text { guidelines, both at a } \\
\text { general level along with } \\
\text { specific categories } \\
\text { - Guideline to evaluate a } \\
\text { process (using existing } \\
\text { methods) } \\
\text { - Non-prescriptive } \\
\text { methodology best practice } \\
\text { guidance (generate ideas } \\
\text { rather than prescribe) } \\
\text { - Guideline to prioritize } \\
\text { identified, observable } \\
\text { (current or future), process } \\
\text { functional failures/ pain } \\
\text { points }\end{array}$ & $\begin{array}{l}\text { - Tools to help } \\
\text { users draw a line } \\
\text { from normal to } \\
\text { abnormal health } \\
\text { conditions based } \\
\text { upon data } \\
\text { - Understanding } \\
\text { of life cycles, } \\
\text { frequency, } \\
\text { resolution and } \\
\text { type of data that } \\
\text { gives health } \\
\text { insights } \\
\text { - Improvement }\end{array}$ \\
\hline 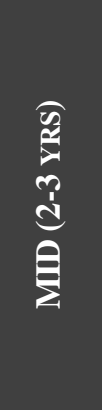 & $\begin{array}{l}\text { Assess state/events (of machine and data quality) } \\
\text { and context } \\
\text { - Devise and conduct adaptive data collection } \\
\text { strategies; include changes in process and } \\
\text { equipment relative to health (e.g., inputs from } \\
\text { modeling) } \\
\text { - Determine data that needs to be captured and type } \\
\text { of tools available to capture, process and visualize } \\
\text { the data } \\
\text { - Develop health data categories } \\
\text { - Test guidelines with pilot program }\end{array}$ & $\begin{array}{l}\text { - Identification of benefits } \\
\text { from access to data } \\
\text { - Process data along with } \\
\text { corresponding part quality } \\
\text { data }\end{array}$ & $\begin{array}{l}\text { spectrum of } \\
\text { parameters) } \\
\text { - Improvement } \\
\text { path } \\
\text { - Methods/data to } \\
\text { enable users to } \\
\text { identify } \\
\text { priorities for } \\
\text { health }\end{array}$ \\
\hline
\end{tabular}

\section{Stakeholders \& POTential Roles}

Industry: Manufacturers - Small, medium, and large; operators, maintenance personnel, PHM system developers and technology developers, technology integrators, process engineers

Association/Trade Groups: OSHA, regulatory authorities; IEEE, PHM Society

Academia: Those involved in industrial and process engineering R\&D, and data collection

Standards Development Organizations: ASME, SAE, ASTM, ISO 108, International Organization of Legal Metrology (OIML), International Electrotechnical Commission (IEC), Korean Standards Association (KSA), Technischer Uberwachungsverein (TUV - German UL) - convene, organize, and identify what's been done

Government: Laboratories - provide technical basis; organize best practices, devise measurements

\section{Contributing Authors and Potential Project Team/Committee Memiers}

Mark Walker, D2K

Jim Daggon, Rice Lake

Donnie Alonzo, ASME

Madhusudanan Navinchandran, NIST
Brian A. Weiss, NIST

Thurston Sexton, NIST

KC Morris, NIST 


\section{Priority Topic 2 Notes}

This guideline or standard should be clear so it is usable by many organizations. Industry personnel appreciate manuals that they can hand off without excess explanation. The guideline should consider physical and configuration data, along with action plans. Asbuilt information is relatively easy to attain (e.g., from original equipment manufacturers) and should include:

a. Statistical likelihoods of failure

b. Expected equipment lifetime

c. Historical/typical use, including:

i. Frequency and how equipment is used

ii. Maintenance of equipment (which should also be an input to health) including trouble-shooting faults and repairing failures, preventive maintenance activities, and integrated predictive maintenance capabilities, if available

iii. Intended future uses

Sensors are an important aspect. The guidance should help users understand what sensors and software they need to purchase based on the level of certainty required. How good does a sensor need to be for a specific use? This context is important. Further, the quality of the data relies on both the overall capability and health of the sensors.

While process improvements may look at equipment failure, health improvement also looks at equipment function. What causes functions to fail? Does the guidance cover technology integration with existing/legacy equipment, PHM technology built-in to new equipment, or both? Do these situations require different guidance documents? These questions will need answers.

The standard/guideline document should also consider economics (e.g., optimizing ROI). From the industry perspective, PHM is best justified if associated with attractive financial implications. Ease of implementation should also be factored into these guidelines as that will have a financial impact on the adoption of new technologies. 
Figure 7: Priority Guideline Action Plan for Topic 5 - Guideline to Determine Where and When PHM Should be Added/Integrated

DESCRIPTION: The envisioned guideline will inform the manufacturing community as to where within a factory PHM should be included and when it should be enabled. This will link common use cases with cost/benefits, quality improvements, and other criteria to inform decisions on addition and integration of PHM into processes and equipment. It is crucial for manufacturers to be aware of the various use cases and configurations that are more prone to failure and/or critical to operations so that they can be effectively monitored to minimize unplanned maintenance and optimize planned maintenance.

\begin{tabular}{|c|c|c|c|}
\hline TIME & $\begin{array}{c}\text { GUIDELINE ACTION PLAN } \\
\text { STEPS }\end{array}$ & $\begin{array}{c}\text { MILESTONES/KEY } \\
\text { DELIVERABLES }\end{array}$ & $\begin{array}{c}\text { PerformanCE } \\
\text { TARGETS }\end{array}$ \\
\hline 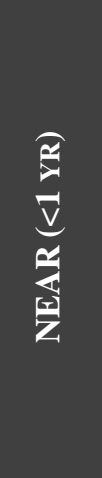 & $\begin{array}{l}\text { Develop matrix of common } \\
\text { operational use cases/needs (e.g., } \\
\text { minimize downtime) } \\
\text { - Define roles for the organizational } \\
\text { functions responsible for PHM system } \\
\text { - Define approach for conducting gap } \\
\text { analysis to determine physical and } \\
\text { informational requirements to support } \\
\text { PHM and gather baseline data } \\
\text { - Define approach for performing cost } \\
\text { and return-on-investment (ROI) } \\
\text { analysis for common use cases }\end{array}$ & $\begin{array}{l}\text { - White paper on cost/benefit } \\
\text { analysis and return on investment } \\
\text { methodology } \\
\text { - Identification of improvements } \\
\text { - Guidelines to promote the } \\
\text { implementation of PHM within a } \\
\text { manufacturer process, highlighting } \\
\text { specific implementation differences } \\
\text { between large and small to } \\
\text { medium-sized organizations } \\
\text { - Index of quality improvement that } \\
\text { links between PHM and impact to } \\
\text { quality - whitepaper }\end{array}$ & \multirow{3}{*}{$\begin{array}{l}\text { - Documentation of } \\
\text { current baseline } \\
\text { PHM capabilities } \\
\text { and justification } \\
\text { for PHM based } \\
\text { upon operational } \\
\text { use cases and } \\
\text { performance } \\
\text { criteria }\end{array}$} \\
\hline 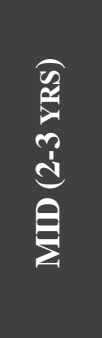 & $\begin{array}{l}\text { Define process for characterizing } \\
\text { assets for data collection including } \\
\text { different aspects of process } \\
\text { - Define approach for assessing impacts } \\
\text { of company maturity on PHM } \\
\text { addition/integration } \\
\text { - Define approach for assessing impact } \\
\text { of PHM addition/integration on } \\
\text { company maturity }\end{array}$ & & \\
\hline 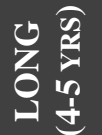 & $\begin{array}{l}\text { - Develop process for change } \\
\text { management, i.e., "what to do with } \\
\text { the data" as it accumulates }\end{array}$ & & \\
\hline
\end{tabular}

\section{Stakeholders \& Potential Roles}

Industry: OEM "large and small" equipment and sensor manufacturers; IT organization "firms"; software developers

Association/Trade Groups: PHM Society, SME, Vibration Institute, etc.

Academia: IMS; AI and smart manufacturing initiatives

Standards Dev. Organizations: ASME, SAE, IEEE, etc.

Government: NIST, AFRL, NAVAIR, FRC Command Center, NASA

\section{Contributing Authors and Potential Project Team/Committee Memiers}

Al Salour, The Boeing Co.

Prabhu Jacob, Cummins

William Walker, D2K

Luis Hernandez, GSS
Steve Weinman, ASME

Mike Brundage, NIST

Alex Klinger, NIST

Guixiu 'Helen' Qiao, NIST

Logen Johnson, SAE 
Figure 8: Priority Guideline Action Plan for Topic 7 - Guideline to Determine Where PHM should be Performed

DESCRIPTION: Manufacturers need a guideline to provide direction on where to perform PHM, i.e., locally, at the edge, via cloud data processing, etc. and who is responsible for the PHM analyses (e.g., within the organization, external data analysis company). This requires an understanding of the benefits and disadvantage and other requirements for the various options. Guidelines should also provide direction for ownership and governance of the data under these different scenarios.

\begin{tabular}{|c|c|c|c|}
\hline TIME & $\begin{array}{c}\text { GUIDELINE ACTION PLAN } \\
\text { StePS }\end{array}$ & $\begin{array}{c}\text { MILESTONES/KEY } \\
\text { DELIVERABLES }\end{array}$ & $\begin{array}{c}\text { PerformanCE } \\
\text { TARGETS }\end{array}$ \\
\hline 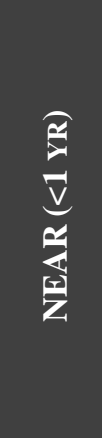 & $\begin{array}{l}\text { Assess the pros and cons of local, } \\
\text { edge, or cloud data processing for } \\
\text { PHM, and ownership and } \\
\text { governance of the data (e.g., who } \\
\text { conducts analysis, has expertise; data } \\
\text { anonymity; who owns the data and } \\
\text { governs it) } \\
\text { Determine how to structure the data } \\
\text { and whether data should be real time, } \\
\text { historical, etc. } \\
\text { Determine how much data is needed } \\
\text { and how it should be structured }\end{array}$ & $\begin{array}{l}\text { - Guideline to determine cost } \\
\text { for implementing where } \\
\text { PHM analyses should be } \\
\text { performed } \\
\text { - } \quad \text { Guideline on where the data } \\
\text { should be stored } \\
\text { - Survey to be conducted from } \\
\text { small, medium, and large } \\
\text { manufacturers and } \\
\text { documented }\end{array}$ & $\begin{array}{l}\text { Guideline that } \\
\text { provides direction for } \\
\text { different scenarios for } \\
\text { PHM performance, } \\
\text { including data criteria, }\end{array}$ \\
\hline 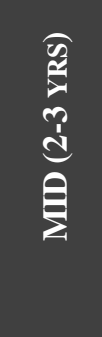 & 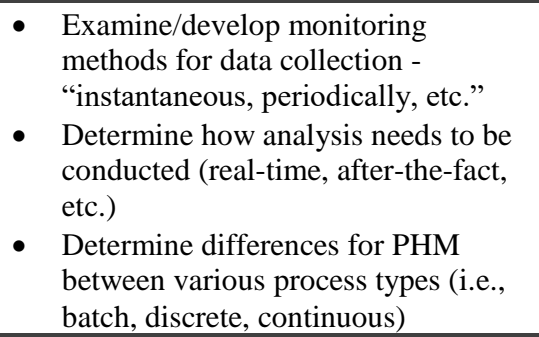 & $\begin{array}{l}\text { - Cost effectiveness of data } \\
\text { acquisition and frequency - } \\
\text { "continuous vs. intermittent" } \\
\text { and the subsequent analysis } \\
\text { - Which type of PHM analysis } \\
\text { should be used considering } \\
\text { feasibility, physical security, } \\
\text { cybersecurity, etc. }\end{array}$ & $\begin{array}{l}\text { structure and } \\
\text { governance }\end{array}$ \\
\hline
\end{tabular}

\section{STAKeholders \& POTENTIAL ROLES}

Industry: Cloud service providers, hardware companies, database and datacenter owners, cybersecurity, data scientists/engineers, PHM analysts

\section{Association/Trade Groups: V4I}

Academia: Data scientists; AI experts

Standards Dev. Organizations: MIMOSA, OASIS, FRC, DoD, V\&V Committee, ISO Committee

Government: NIST, DoD

\section{Contributing Authors and Potential Project Team/Committee Memiers}

Al Salour, The Boeing Co.

Prabhu Jacob, Cummins

William Walker, D2K

Luis Hernandez, GSS

Logen Johnson, SAE
Steve Weinman, ASME

Mike Brundage, NIST

Alex Klinger, NIST

Guixiu 'Helen' Qiao, NIST

Logen Johnson, SAE 


\section{Next Steps}

Following the Industry Forum, NIST researchers are planning multiple efforts to 1) continue the development of the necessary measurement science to verify and validate emerging PHM technologies operating in manufacturing environments, 2) further the engagement of the industrial, academic, and government communities to ensure relevant measurement science products are delivered to the stakeholder community, 3) pilot/use emerging test methods, reference datasets, and software tools to promote further technological development of PHM, and 4) generate standards and guidelines to facilitate greater adoption of both PHM technologies and the methods to assess their capabilities. Anyone who would like additional information on these activities should contact Brian A. Weiss (Leader - Prognostics and Health Management for Reliable Operations in Smart Manufacturing) at brian.weiss@ nist.gov.

Since the conclusion of the Industry Forum and the public release of this document, several activities have either occurred or are actively being planned.

\subsection{ASME Face-to-Face Subcommittee Meetings}

The next face-to-face meeting of this newly-formed ASME Subcommittee was hosted by TechSolve in Cincinnati, Ohio on October $25^{\text {th }}$ and $26^{\text {th }}$, 2018. A majority of the subcommittee was in attendance where the two-day meeting was very productive. Specifically, the three roadmap action plans (presented in Figure 6, Figure 7, and Figure 8) were discussed in further detail where a subset of these plans are being prepared for guideline development. Additionally, another roadmap action plan on terminology was presented to the group for feedback. The next face-to-face subcommittee meeting is being tentatively planned for May 2019 at NIST in Gaithersburg, Maryland. Anyone interested in participating should contact brian.weiss@ nist.gov.

\subsection{ME4PHM}

The Industry Forum organizing committee is planning the next installment of the Industry Forum to occur at the 2019 Annual Conference of the PHM Society ${ }^{19}$ scheduled for the week of September $23^{\text {rd }}, 2019$ in Scottsdale, Arizona. The event is currently being planned as a one-day workshop and will be called the Measurement and Evaluation for PHM for Manufacturing Operations (ME4PHM) Forum to reflect the focus on PHM measurement, evaluation, and standards in the manufacturing domain. The event organizers are actively drafting the agenda. Please contact brian.weiss@ @ist.gov for additional information or if you would like to contribute/participate in this event.

\section{References}

[1] Vogl, G. W., Weiss, B. A., and Helu, M., 2016, "A review of diagnostic and prognostic capabilities and best practices for manufacturing," Journal of Intelligent Manufacturing.

${ }^{19}$ http://www.phmsociety.org/ 
[2] Pellegrino, J., Justiniano, M., Raghunathan, A., and Weiss, B. A., 2016, "Measurement Science Roadmap for Prognostics and Health Management for Smart Manufacturing Systems," NIST Advanced Manufacturing Seriess (AMS).

[3] Weiss, B. A., Vogl, G. W., Helu, M., Qiao, G., Pellegrino, J., Justiniano, M., and Raghunathan, A., 2015, "Measurement Science for Prognostics and Health Management for Smart Manufacturing Systems: Key Findings from a Roadmapping Workshop," Annual Conference of the Prognostics and Health Management Society 2015, P. Society, ed., PHM Society, Coronado, CA, p. 11.

[4] Jin, X., Siegel, D., Weiss, B. A., Gamel, E., Wang, W., Lee, J., and Ni, J., 2016, "The present status and future growth of maintenance in US manufacturing: results from a pilot survey," Manufacturing Rev., 3, p. 10.

[5] Helu, M., and Weiss, B. A., "The current state of sensing, health management, and control for small-to-medium-szed manufacturers," Proc. ASME 2016 Manufacturing Science and Engineering Conference, MSEC2016.

[6] Jin, X., Weiss, B. A., Siegel, D., and Lee, J., 2016, "Present Status and Future Growth of Advanced Maintenance Technology and Strategy in US Manufacturing," International Journal of Prognostics and Health Management, 7 (Special Issue on Smart Manufacturing PHM)(Sp5), p. 18.

[7] Vogl, G. W., Weiss, B. A., and Donmez, M. A., 2014, "Standards Related to Prognostics and Health Management (PHM) for Manufacturing," No. NISTIR 8012, National Institute of Standards and Technology (NIST), Gaithersburg, Maryland, USA.

[8] Vogl, G. W., Weiss, B. A., and Donmez, M. A., "Standards for prognostics and health management (PHM) techniques within manufacturing operations," Proc. Annual Conference of the Prognostics and Health Management Society 2014.

[9] Weiss, B. A., Alonzo, D., and Weinman, S. D., 2017, "Summary Report on a Workshop on Advanced Monitoring, Diagnostics, and Prognostics for Manufacturing Operations." 


\section{Appendix A - Acronyms}

AFRL Air Force Research Laboratory

AI Artificial Intelligence

ALCIM Asset Life-cycle Information Management

AM Additive Manufacturing

ANSI American National Standards Institute

AR Augmented Reality

ASME American Society of Mechanical Engineers

CBM Condition-based Maintenance

CCAM Commonwealth Center for Advanced Manufacturing

CES Complex engineered systems

CMTC California Manufacturing Technology Consulting

CMXG Commodities Manufacturing

CNC Computer Numerical Control

DMDII Digital Manufacturing and Design Integration

DNN Deep Neural Network

DoD Department of Defense

EBITDA Earnings before interest, tax, depreciation, and amortization

EMF Electromagnetic Field

EOL End of Life

ERP Enterprise Resource Planning

FMEA Failure Modes and Effects Analysis

FRC Fleet Readiness Center 
GD\&T Geometric Dimensioning and Tolerancing

GM General Motors

GSS Global Strategic Solutions

HVAC Heating, Ventilation, and Air Conditioning

HMI Human Machine Interface

HRI Human Robot Interaction

IDM Intelligent Device Management

IEC International Electrotechnical Commission

IEEE Institute of Electrical and Electronics Engineers

IMS Center for Intelligent Maintenance Systems

IoT Internet of Things

ISA International Society of Automation

ISO International Organization for Standardization

IT Information Technology

KSA Korean Standards Association

LiDAR Light Detection and Ranging

MBE Model-based Enterprise

MES Manufacturing Execution System

MIMOSA Machinery Information Management Open Systems Alliance

ML Machine Learning

MSEC Manufacturing Science and Engineering Conference

MWO Maintenance Work Order

MxR Mixed Reality

NASA National Aeronautics and Space Administration 
NAVAIR U.S. Navy Naval Air Systms Command

NIST National Institute of Standards and Technology

NLP Natural Language Processing

OEE Overall Equipment Effectiveness

OEM Original Equipment Manufacturer

OIML International Organization of Legal Metrology

OMM On-machine Metrology

OO Object-Oriented

OPC-UA Open Platform Communications - Unified Architecture

PDF Probability Density Function

PF Particle Filter

PHM Prognostics and Health Management

PLC Programmable Logic Controller

PLM Product Lifecycle Management

PM Preventive Maintenance

PRA Probability Risk Assessment

R\&D Research and Development

ROI Return on Investment

RUL Remaining Useful Life

S\&C Standards and Certification

SDO Standards Development Organization

SME Society of Manufacturing Engineers

SME Subject Matter Expert 
SMM Small and Medium-sized Manufacturer

STEM Science, Technology, Engineering, and Math

TRL Technology Readiness Level

TUV Technischer Uberwachungsverein (German) - Technical Inspection Association

$\mathbf{V} \& \mathbf{V} \quad$ Verification and Validation

V4I Virtual, Verification, Validation, \& Visualization Institute

VR Virtual Reality

WR-ALC Warner Robins Air Logistics Complex 


\section{Appendix B - Industry Forum Conference Program}




\section{Industry Forum:}

Moving from "React and Repair" to "Predict and Prevent"

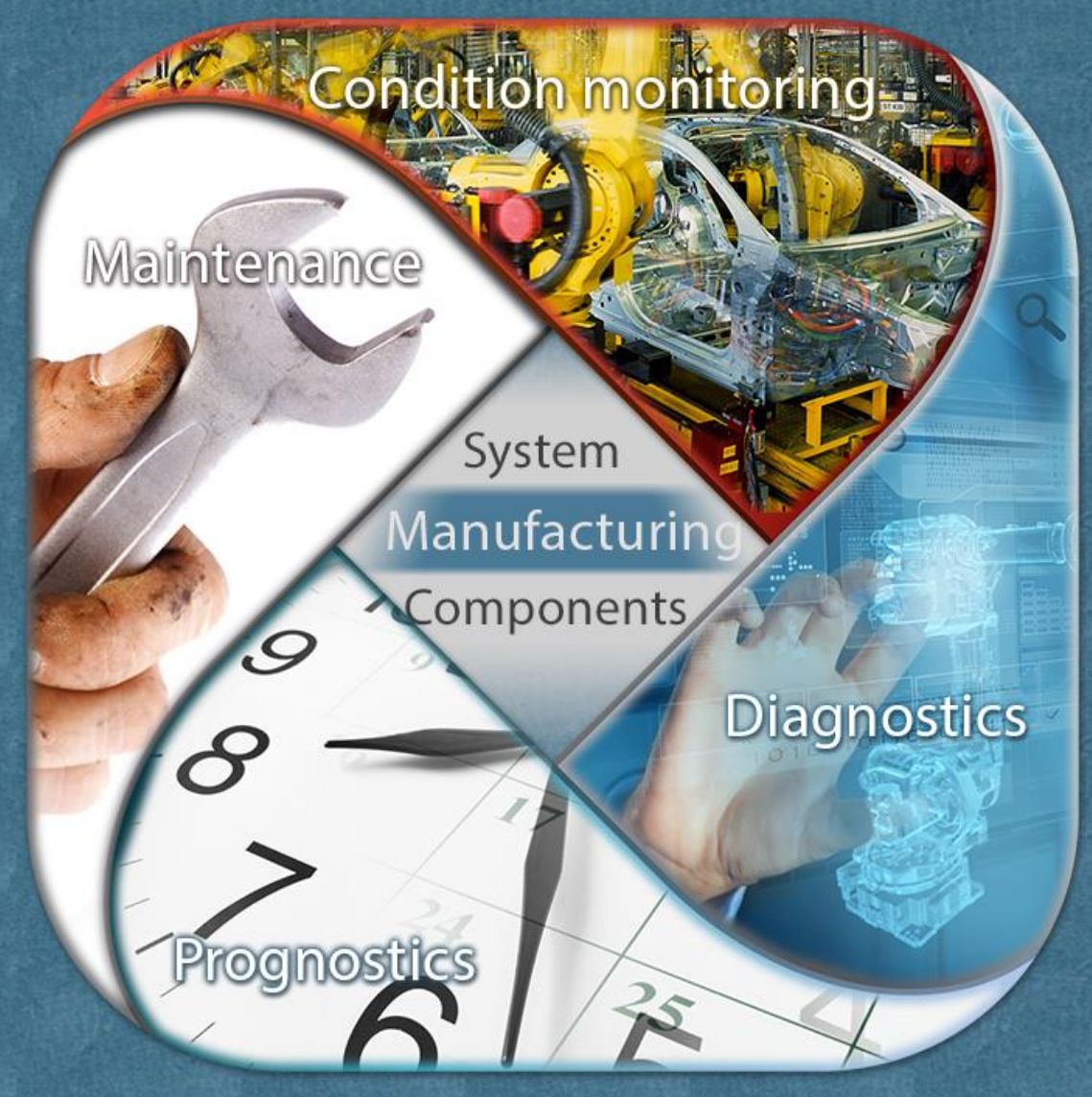

NIST, Gaithersburg, Maryland, USA May 8 - May 11, 2018

\section{Join our Twitter Chat \#IFNIST}




\section{Industry Forum:}

Moving from: "React and Repair" to "Predict and Prevent"

May $8-11,2018$

\section{Welcome}

Welcome to the first-ever Industry Forum: Monitoring, Diagnostics, and Prognostics at the National Institutes of Standards and Technology (NIST). We are pleased that you are joining us for what we expect will be an insightful and productive week discussing current trends, successes, challenges, and needs with respect to advanced monitoring, diagnostic, and prognostic technologies to enhance maintenance and control strategies within manufacturing operations.

The manufacturing community has been evolving as new technologies emerge, existing technologies mature, and advanced technologies become accessible to more organizations as costs decrease and integration challenges are addressed. New fault and failure modes emerge as technologies are integrated into an organization's manufacturing process for the first time, existing processes are being reconfigured to support new products, new sensors are gathering more information than ever before, and analytics offer greater intelligence and awareness. Manufacturers take different approaches, especially between large and small to medium-sized enterprises (SMEs), to deal with these faults and failures. Most manufacturers aim to avoid reactive maintenance - fix equipment after it breaks and interrupts production - as this strategy can present substantial risk and cost. Nearly every manufacturer employs some form of preventive maintenance - change the proverbial oil every 3 months or 3,000 miles - as a way to keep their systems and processes operating within specification. The downside to this strategy is that it sometimes leads to unnecessary cost and downtime if maintenance is done too frequently and it still does not eliminate the prospect of reactive maintenance. Pockets of the manufacturing community are increasing their predictive maintenance capability - plan maintenance activities based upon analyzing specific sensor data that inform upon system and process performance and health - to optimize (ideally) their maintenance activities to minimize both downtime and maintenance costs. Proactive maintenance, intelligent maintenance, and autonomous maintenance are emerging maintenance strategies that present tremendous potential to further minimize equipment and process downtime. With all of these strategies, where each presents its own benefits and challenges, where should a manufacturer begin? No two manufacturers are alike making it unlikely that a single solution will solve every maintenance challenge.

We have designed this Industry Forum to offer you an opportunity to hear directly from the diverse stakeholders who see the value in advancing monitoring, diagnostic, and prognostic technologies. The first three days of presentations and panels will feature various perspectives including: Large to small manufacturers will present on their successes and challenges with respect to their maintenance strategies; technology integrators and technology developers will highlight emerging hardware and software capabilities to enhance awareness; and researchers will discuss how their break-throughs in emerging technology, and verification and validation techniques will expand the boundaries as to what is possible for monitoring equipment and process health in the factory. 


\section{Industry Forum:}

Moving from: "React and Repair" to "Predict and Prevent"

May $8-11,2018$

In addition to our presentations and panel sessions, we have lined up three outstanding plenary talks:

- Michael Molnar, Founding Director of the Office of Advanced Manufacturing, will present on how Manufacturing USA is creating vast research networks to solve some of the industry's most challenging problems.

- Albert Wavering, Chief of the Intelligent Systems Division, will share NIST's smart manufacturing research efforts focused on developing measurement technologies, performance metrics, test methods, and tools to enhance industry competitiveness.

- Jaime Camelio, Chief Technology Officer of the Commonwealth Center for Advanced Manufacturing (CCAM), will discuss process monitoring and diagnosis as he works to build up Virginia's manufacturing capabilities with CCAM industrial partners.

The final day of the Forum, Friday, will be devoted to building up an ASME-led standards community focused on advancing monitoring, diagnostics, and prognostics for manufacturing operations. ASME and NIST personnel will guide the participants in examining specific priority areas and detailing the next steps to generate and deliver guidelines to industry that enhance a manufacturer's ability to design, deploy, verify, and validate their maintenance-related capabilities. We hope you can participate with us in this standards effort.

The entire four-day Industry Forum will be summarized in a report that is expected for public release later in 2018. Even if you cannot stay with us for the duration of the event, you can still stay informed on the forum's output. Likewise, as we actively build up this standards community, we are seeking additional volunteers to offer their time and expertise.

Whether you are from industry, academia, or government, we are confident you will enjoy your week at NIST.

- $\quad$ Brian A. Weiss, Intelligent Systems Division, NIST

- $\quad$ Michael P. Brundage, Systems Integration Division, NIST 


\section{Industry Forum:}

Moving from: "React and Repair" to "Predict and Prevent" May $8-11,2018$

\section{THANK YOU to our SPONSORS}

Gold Sponsors

OEE Data Watch, LLC

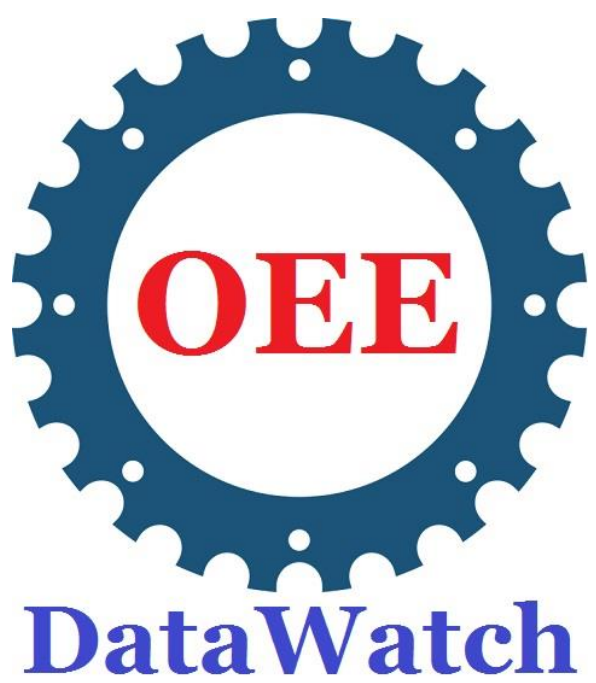

Silver Sponsors

ASME

D2K Technologies

SAE

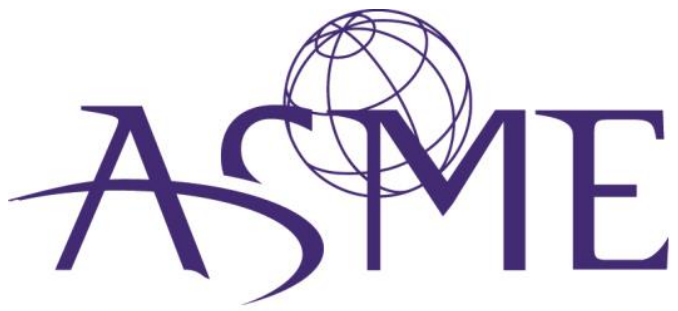

SETTING THE STANDARD
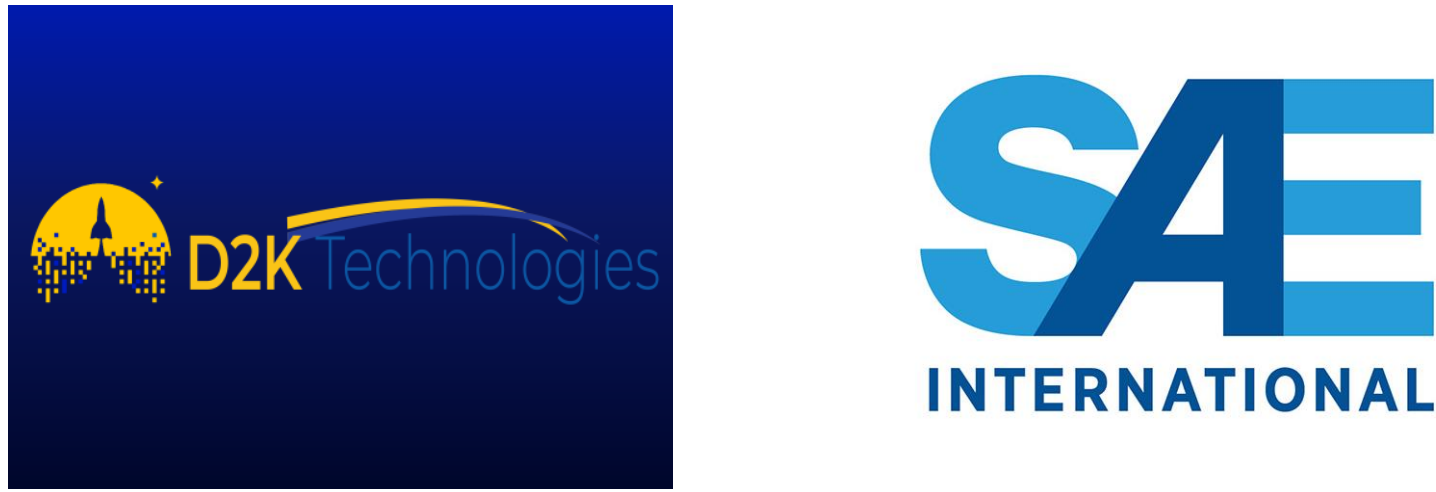

INTERNATIONAL $\circledast$ 


\section{Ind ustry Forum: Monitoring, Dia gnostics, and Prognostics for Ma nufacturing Operations}

MOVING from "REACT and REPAIR" to "PREDICT and PREVENT"

\begin{tabular}{|c|c|c|}
\hline & \multicolumn{2}{|c|}{ AGENDA: Tuesday, May 8, 2018 (Green Auditorium) } \\
\hline TIME & EVENT/SESSION & PRESENTERS \\
\hline $7: 15-4: 30$ & Registration (outside the auditorium) & \\
\hline $8: 15-8: 30$ & Introduction and Safety & $\begin{array}{l}\text { Brian A. Weiss (NIST - Intelligent Systems Division), Michael Brundage (NIST - Systems } \\
\text { Integration Division) }\end{array}$ \\
\hline $8: 30-8: 45$ & Overview of NIST's Engineering Laboratory and Welcome & Kirk Dohne (NIST - Engineering Laboratory) \\
\hline $8: 45-9: 20$ & $\begin{array}{l}\text { PLENARY: Manufacturing USA: Solving Tough Industry } \\
\text { Challenges Through Collaboration }\end{array}$ & Michael Molnar (NIST - Office of Advanced Manufacturing) \\
\hline 9:20-9:35 & BREAK & \\
\hline $9: 35-11: 25$ & $\begin{array}{l}\text { Large Manufacturing Needs and Case Studies - Presentations } \\
\& \text { Panel }\end{array}$ & $\begin{array}{l}\text { Al Salour (Boeing), Luis Hernandez (Global Strategic Solutions), James Moyne (Applied } \\
\text { Global Services) }\end{array}$ \\
\hline $11: 25-12: 40$ & LUNCH & \\
\hline $12: 40-2: 30$ & $\begin{array}{l}\text { Small to Medium Manufacturing Needs and Case Studies - } \\
\text { Presentations \& Panel }\end{array}$ & $\begin{array}{l}\text { Scott Sipe (Mantec), Tom Zbell (Genedge), Thorsten Wuest (West Virginia University), } \\
\text { Mark Walker (D2K) }\end{array}$ \\
\hline $2: 30-2: 45$ & BREAK & \\
\hline $2: 45-4: 35$ & $\begin{array}{l}\text { Communication and Information Flow to support PHM - } \\
\text { Presentations \& Panel }\end{array}$ & $\begin{array}{l}\text { Will Sobel (Vimana), Moneer Helu (NIST - Systems Integration Division), Joel Neidig } \\
\text { (ITAMCO), Rob Andes (The Knowledge Design Company) }\end{array}$ \\
\hline $4: 35-4: 55$ & $\begin{array}{l}\text { The Costs and Benefits of Advanced Maintenance in } \\
\text { Manufacturing }\end{array}$ & Doug Thomas (NIST - Applied Economics Office) \\
\hline $4: 55-5: 00$ & Closing and Departure & Brian A. Weiss (NIST - Intelligent Systems Division) \\
\hline
\end{tabular}




\section{AGENDA: Wednesday, May 9, 2018 (Green Auditorium)}

\begin{tabular}{|c|c|c|}
\hline TIME & EVENT/SESSION & PRESENTERS \\
\hline 7:00-4:30 & Registration (outside the auditorium) & \\
\hline 8:00-8:05 & Welcome & $\begin{array}{l}\text { Brian A. Weiss (NIST - Intelligent Systems Division), Michael Brundage (NIST - Systems Integration } \\
\text { Division) }\end{array}$ \\
\hline $8: 05-8: 35$ & $\begin{array}{l}\text { PLENARY: NIST Smart Manufacturing Programs: Driving } \\
\text { Innovation and Reducing Risks of Adoption of New } \\
\text { Technologies }\end{array}$ & Al Wavering (NIST - Intelligent Systems Division) \\
\hline $8: 35-10: 35$ & $\begin{array}{l}\text { Emerging Sensing Technologies to Enable Monitoring, } \\
\text { Diagnostics, and Prognostics - Presentations \& Panel }\end{array}$ & $\begin{array}{l}\text { Radu Pavel (TechSolve), Brittany Newell (Purdue University), Justinian Rosca (Siemens } \\
\text { Corporation), Gregory Vogl (NIST - Intelligent Systems Division), Ed Spence (Machine } \\
\text { Instrumentation) }\end{array}$ \\
\hline 10:35 - 10:50 & BREAK & \\
\hline 10:50 - 12:50 & $\begin{array}{l}\text { Planning and Assessment to Promote Monitoring, Diagnostic, } \\
\text { and Prognostic Technologies - Presentations \& Panel }\end{array}$ & $\begin{array}{l}\text { Karl Reichard (Penn State University Applied Research Lab), Ananth Seshan (MESA), Kai Goebel } \\
\text { (NASA), Miguel Saez (University of Michigan), Jorge Arinez (General Motors) }\end{array}$ \\
\hline $12: 50-1: 50$ & LUNCH & \\
\hline $1: 50-3: 20$ & $\begin{array}{l}\text { Monitoring and Analysis Technologies for Prognostics and } \\
\text { Health Management (PHM) - Presentations }\end{array}$ & $\begin{array}{l}\text { David Siegel (Predictronics), Nancy Diaz-Elsayed (University of South Florida), Sanket Amberkar } \\
\text { (Falkonry), Robert Gao (Case Western Reserve University), ChaBum Lee (Tennessee Tech } \\
\text { University) }\end{array}$ \\
\hline 3:20-3:35 & $B R E A K$ & \\
\hline $3: 35-4: 05$ & $\begin{array}{l}\text { Monitoring and Analysis Technologies for Prognostics and } \\
\text { Health Management (PHM) - Panel }\end{array}$ & $\begin{array}{l}\text { David Siegel (Predictronics), Nancy Diaz-Elsayed (University of South Florida), Sanket Amberkar } \\
\text { (Falkonry), Robert Gao (Case Western Reserve University), ChaBum Lee (Tennessee Tech } \\
\text { University) }\end{array}$ \\
\hline $4: 05-4: 25$ & Emerging Research Efforts & Junmin Lee (Seoul National University), Chan Hee Park (Seoul National University) \\
\hline $4: 25-4: 45$ & $\begin{array}{l}\text { Using Unstructured Work Order Data to Improve Maintenance } \\
\text { Procedures in Manufacturing }\end{array}$ & Michael Brundage (NIST - Systems Integration Division) \\
\hline $4: 45-5: 05$ & $\begin{array}{l}\text { NIST Research on Monitoring, Diagnostics, and Prognostics for } \\
\text { Manufacturing Workcells }\end{array}$ & Brian A. Weiss (NIST - Intelligent Systems Division) \\
\hline $5: 05-5: 10$ & Closing and Departure & $\begin{array}{l}\text { Brian A. Weiss (NIST - Intelligent Systems Division), Michael Brundage (NIST - Systems Integration } \\
\text { Division) }\end{array}$ \\
\hline
\end{tabular}




\section{AGENDA: Thursday, May 10, 2018 (Green Auditorium)}

\begin{tabular}{|c|c|c|}
\hline TIME & EVENT/SESSION & PRESENTERS \\
\hline $7: 30-4: 30$ & Registration (outside the auditorium) & \\
\hline $8: 30-8: 35$ & Welcome Address & $\begin{array}{l}\text { Brian A. Weiss (NIST - Intelligent Systems Division), Michael Brundage (NIST - Systems } \\
\text { Integration Division) }\end{array}$ \\
\hline $8: 35-9: 05$ & $\begin{array}{l}\text { PLENARY: CCAM Activities in Process Monitoring and } \\
\text { Diagnosis }\end{array}$ & Jaime Camellio (Commonwealth Center for Advanced Manufacturing) \\
\hline 9:05 - 10:17 & Standards and Best Practices - Presentations & $\begin{array}{l}\text { Andrew Hess (Hess PHM Group), Ravi Rajamani (drR2), Tom Fiske (Yokogawa), Tom } \\
\text { Hedberg (NIST - Systems Integration Division) }\end{array}$ \\
\hline 10:17 - 10:30 & BREAK & \\
\hline 10:30 - 10:50 & Standards and Best Practices - Presentations Cont. & Logen Johnson (SAE), Donnie Alonzo (ASME) \\
\hline 10:50 - 11:50 & Standards and Best Practices - Panel & $\begin{array}{l}\text { Andrew Hess (Hess PHM Group), Ravi Rajamani (drR2), Tom Fiske (Yokogawa), Tom } \\
\text { Hedberg (NIST - Systems Integration Division), Logen Johnson (SAE), Donnie Alonzo } \\
\text { (ASME) }\end{array}$ \\
\hline 11:50 - 1:00 & LUNCH & \\
\hline 1:00 - 2:15 & $\begin{array}{l}\text { PHM within the International Manufacturing Community - } \\
\text { Presentations }\end{array}$ & $\begin{array}{l}\text { Byeng Youn (Seoul National University), Hyunbo Cho (Pohang University of Science and } \\
\text { Technology), Hyunseok Oh (Gwangju Institute of Science and Technology) }\end{array}$ \\
\hline $2: 15-3: 15$ & Visualization Tools for PHM - Presentations \& Panel & Jeremy Marvel (NIST - Intelligent Systems Division), Sinan Bank (Siemens Corporation) \\
\hline $3: 15-3: 30$ & BREAK & \\
\hline $3: 30-4: 00$ & Demystifying Today's Al & Michael Garris (NIST - Information Technology Laboratory) \\
\hline 4:00 - 4:30 & $\begin{array}{l}\text { Industry Al-- A System Perspective in Machine Learning for } \\
\text { Smart Manufacturing and Maintenance }\end{array}$ & Jay Lee (University of Cincinnati, Center for Intelligent Maintenance Systems) \\
\hline $4: 30-5: 00$ & $\begin{array}{l}\text { Planning for the Future - Building and Leveraging Artificial } \\
\text { Intelligence: Panel Discussion }\end{array}$ & $\begin{array}{l}\text { Jay Lee (University of Cincinnati, Center for Intelligent Maintenance Systems), Michael } \\
\text { Garris (NIST - Information Technology Laboratory) }\end{array}$ \\
\hline $5: 00-5: 10$ & Closing and Departure & $\begin{array}{l}\text { Brian A. Weiss (NIST - Intelligent Systems Division), Michael Brundage (NIST - Systems } \\
\text { Integration Division) }\end{array}$ \\
\hline
\end{tabular}




\section{AGENDA: Friday, May 11, 2018 (Green Auditorium)}

\section{ASME Standards Meeting - Monitoring, Diagnostics, and Prognostics for Manufacturing Operations}

8:05 - 8:15 - Introduction to ASME's Efforts in Monitoring, Diagnostics, and Prognostics (Brian Weiss / Michael Brundage)

- Motivation for building up this standards community

- $\quad$ Highlighted gaps from June and October 2017 Workshops

June 2017 Workshop Report - https://nvlpubs.nist.gov/nistpubs/ams/NIST.AMS.100-13.pdf

8:15 - 8:30 - Introduction to ASME (Donnie Alonzo/Steve Weinman)

8:30 - 9:00 - Presentation and collection of comments and feedback on Draft Charter for New Subcommittee (Brian 9:10 - 10:00 - BRAINSTORMING \#1 Discussion on Areas of Priority / Subcommittee Work Breakdown

This session will feature discussion on the top (6) priority areas that were identified in prior workshops and a

determination which of these (6) areas should be discussed in greater detail in the BRAINSTORMING \#2

- $\quad$ Standardized Terminology for PHM Guideline on Data and Collection Strategies

- $\quad$ Guideline to Determine What Health Data to Capture and Collection Strategies to Employ

- $\quad$ Guideline to Determine What Sensors and Where they should be deployed to inform on Process/Equipment

- $\quad$ Guideline for implementing sensor data fusion/multi-modal data fusion

- $\quad$ Guideline to Determine When and Where PHM should be added/integrated

- $\quad$ Expand MTConnect/Data Communications

10:00 - 10:15- BREAK

10:15 - 12:30 - BRAINSTORMING \#2 - Work Breakdown Structures and Project Team / Committee Structure

Groups will be formed and will be tasked to further detail a priority area (noted above). This effort will include

definition/clarification of key deliverables, specific tasks, and an estimated timeline.

12:30 - 1:30- LUNCH

1:30 - 2:15 - BRAINSTORMING \#3 - Report Back, Interested Parties and Identification of potential Members / Finalizing of

Committee Structure and Breakdown

2:15 - 2:30 - RECAP FROM ASME ON NEXT STEPS AND REQUIRED ITEMS FOR MEMBERSHIP (Donnie Alonzo/Steve

2:30 - 2:45 - CLOSING AND DEPARTURE (Brian Weiss, Michael Brundage) 


\section{Industry Forum:}

Moving from: "React and Repair" to "Predict and Prevent"

May $8-11,2018$

\section{Tuesday, May 8, 2018}

\section{Overview of NIST's Engineering Laboratory and Welcome}

Kirk Dohne, Engineering Laboratory, National Institute of Standards and Technology (NIST)

Bio: Kirk Dohne is the Associate Director of the National Institute of Standards and Technology's (NIST's) Engineering Laboratory, which addresses the measurement and standards needed to support technology-intensive manufacturing, construction, building energy efficiency, and smart grid and cyber-physical systems. The Engineering Laboratory also conducts research to reduce the risks of fire, earthquakes, tornadoes, and other natural and manmade hazards.

Kirk came to NIST in 2001 to assist the Information Technology Laboratory in strategic planning and impact analysis. He then moved to the Engineering Laboratory in 2012. The Engineering Laboratory has staff of approximately 400 people, distributed among five major research divisions, including two divisions primarily focused on advanced manufacturing - the Intelligent Systems Division and the Systems Integration Division. The laboratory's annual budget is nearly $\$ 100$ million.

\section{PLENARY - Manufacturing USA: Solving Tough Industry Challenges Through \\ Collaboration}

Michael Molnar, Office of Advanced Manufacturing, NIST

Bio: Mike Molnar is the founding director of the Office of Advanced Manufacturing at the National Institute of Standards and Technology, and the Advanced Manufacturing National Program Office, an interagency team which serves as the Congressionally-designated program office for Manufacturing USA - the National Network for Manufacturing Innovation.

Mike joined NIST in 2011. Prior to federal service, Mike had a nearly 30-year industry career in advanced manufacturing, with leadership roles in manufacturing technology development, corporate manufacturing engineering, capital planning, metrology, quality systems, robotics and flexible automation, and computer integrated manufacturing systems. Mid-career Mike served as the manufacturing policy Fellow in the White House Office of Science and Technology Policy.

Mike has been active in engineering professional societies for over thirty years, holding over 70 elected or appointed leadership positions - including President of the Society of Manufacturing Engineers and now Governor of the American Society of Mechanical Engineers. He is a licensed Professional Engineer, Certified Manufacturing Engineer, and was elected Fellow of both ASME and SME. He was recently recognized with the Golden Eagle award from the Boy Scouts of America and the Merchant Manufacturing Medal of ASME/SME. 


\section{Industry Forum:}

Moving from: "React and Repair" to "Predict and Prevent"

May $8-11,2018$

\section{Large Manufacturing Needs and Case Studies - Presentations \& Panel}

Al Salour, The Boeing Company

Presentation Title: Monitoring, Diagnostics, and Prognostics for Large Manufacturing Operations

Bio: Dr. Salour is a Boeing Technical Fellow and the enterprise leader for Network Enabled Manufacturing technologies. He is responsible for a systems approach to develop, integrate, and implement affordable sensor-based manufacturing strategies and plans that provide real time data for factory systems and supplier networks. He is building a model for the current and future Boeing factories by streamlining and automating data management to reduce factory direct labor and overhead support, and promote manufacturing as a competitive advantage. Dr. Salour is a research investigator with national and international premiere universities and research labs. He is a member of the Industrial wireless technical working group with the NIST. Dr. Salour has 30 invention disclosures, 20 patents and 1 trade secret in manufacturing technologies.

\section{Luis Hernandez, Global Strategic Solutions}

Presentation Title: Condition Based Maintenance in DoD - Are we there yet?

Bio: Luis Hernandez is the Managing Director at Global Strategic Solutions LLC. He has 30 years of experience in diagnostic equipment systems engineering along with over 10 years of experience in Integrated Vehicle Health Management (IVHM)/Prognostics and Health Management (PHM) systems applied research. He is actively leading his organization's contributions to the SAE HM-1 standards development efforts (e.g., JA6268). He holds a B.S. Electrical Engineering degree that he received from Wayne State University and participated in the MBA program at Cal State in Los Angeles.

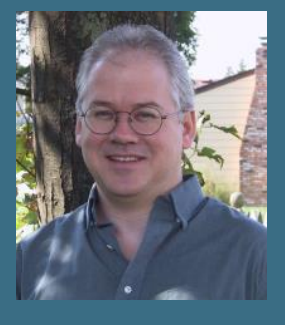

James Moyne, Applied Global Services

Presentation Title: A Solution Roadmap for Moving from Reactive to Prognostic Technologies in Semiconductor Manufacturing

Bio: James Moyne is a consultant for standards and technology to the Applied Global Services group at Applied Materials, and an Associate Research Scientist at the University of Michigan, where he received his Ph.D. degree. Dr. Moyne has experience in advanced process control, prediction technology (predictive maintenance, virtual metrology, and yield prediction), and big data technology, focusing on analytics; he is the author of a number of refereed publications and holds patents in each of these areas. He currently cochairs the Factory Integration Thrust of the International Roadmap for Devices and Systems (IRDS), and well as a number of semiconductor manufacturing standards efforts (SEMI). 


\section{Industry Forum:}

Moving from: "React and Repair" to "Predict and Prevent"

May $8-11,2018$

\section{Small and Medium Manufacturing Needs and Case Studies - Presentations \& Panel}

\section{Scott Sipe, Mantec}

Presentation Title: The Connected Factory for SMM-Opportunities and Challenges

Bio: Scott W. Sipe is the Director of Finance and Technology for MANTEC and has been with the company since January 1998. His responsibilities include oversight of the organization's finances, grants and contracts and the corporate information systems. Scott also delivers information technology planning and consulting services to the manufacturers in the MANTEC region. These services include business process analysis and selection facilitation oriented towards business software, network infrastructure assessment and planning, technology security assessment and planning, implementing broadband strategies, technology strategic planning and technical training.

Before joining MANTEC, Scott spent many of his years working in the manufacturing and distribution industry. Scott holds a degree in Business and Finance and is also CompTIA Network + Certified, Microsoft Certified Professional, Microsoft Certified Engineer and a Microsoft Certified Trainer.

\section{Tom Zbell, Genedge}

Presentation Title: Overall Case Studies and Perspectives - Small and Medium-sized Manufacturers in Virginia

Bio: Tom Zbell is a project manager and lean practice manager at Genedge Alliance. He has demonstrated consulting capabilities in Lean Enterprise Implementation, Office Lean, Lean Six Sigma, Equipment design/Process Engineering, Total Productive Maintenance Systems, Kaizen, Continuous Improvement, and Safety/Environmental Services. Some of his past clients have included the U.S. Navy, U.S. Coast Guard, BAE Systems, Newport News Ship Building, AMF Bowling, Mead Westvaco, Boehringer Ingelheim Chemicals Inc., ABB, Delta Star, Church \& Dwight, Lutron, American Red Cross, Sperry Marine, Trex and Wyeth. Mr. Zbell has a MS Organizational Management from Central Connecticut State University, a BS Manufacturing Engineering from Utah State University, and an AS Manufacturing Engineering, Waterbury State Technical College.

\section{Thorsten Wuest, West Virginia University}

\section{Presentation Title: Smart Manufacturing for SMM - Opportunities and Challenges}

Bio: Dr. Thorsten Wuest is a faculty member and J. Wayne and Kathy Richards Faculty Fellow in the IMSE Department at WVU and head of WVU's Smart Manufacturing Lab. He serves as the inaugural IDEA Fellow at Statler College to include principles of innovation, design and entrepreneurship. His academic and professional background reflects his interdisciplinary research and teaching interest with Masters degrees in International Business (New Zealand) and Industrial Engineering and Management as well as a PhD in Production Engineering (Summa Cum Laude), both from the University of Bremen, Germany. He worked as a research scientist for BIBA, Germany ('09-'15) with previous roles incl. German Armed Forces (Lieutenant), Arthur D. Little (Switzerland) and ThyssenKrupp Technologies (Germany). Over the last years, Dr. Wuest successfully acquired several externally funded grants (Industry, DFG, EC, BMBF, etc.) and received several awards for his work, among them multiple best paper awards and an outstanding dissertation award. 


\section{Industry Forum:}

Moving from: "React and Repair" to "Predict and Prevent"

May $8-11,2018$

Dr. Wuest is a research affiliate of the CIRP, member of IFIP WG 5.1 \& 5.7, senior member of IISE as well as in the core team of the World Manufacturing Forum (WMF). He is an associate editor for the International Journal of Manufacturing Research (IJMR) and serves in the editorial board of the Journal of Manufacturing Systems (JMSY). Dr. Wuest published over 80 peer-reviewed articles in international archival journals and conferences and serves as a reviewer for many. Dr. Wuest's research focus is on smart manufacturing, (closed-loop) product lifecycle management (PLM), data analytics in manufacturing, (I)loT, product service systems (PSS) and related issues like interoperability between systems and SME specific challenges. In his research, Dr. Wuest aims to create impact for industry and add value for all stakeholders involved.

\section{Mark Walker, D2K Technologies}

Presentation Title: Crafting Intelligent Systems Management Using Requirements-Driven Design

Bio: Mark Walker received his BSEE from Cal Poly University, Pomona (1990), and his MSCompEng from the University of Southern California, Los Angeles, CA (1994), where he specialized in machine intelligence. Prior to his education, he was trained and served as a Nuclear Reactor Operator onboard U.S.S. Long Beach, CGN-9. His experience in artificial intelligence began in 1989 as a DOE undergraduate fellow at the Center for Engineering and Science Advanced Research Lab at Oak Ridge National Laboratory where he developed image processing and perception software for autonomous robots. His work with HUMS and PHM began in 1996 with BFGoodrich Aerospace, Vergennes, VT, where he developed onboard health and state estimation algorithms for the Joint Strike Fighter, and coauthored four patents in applied artificial intelligence. He also spent 6 years as Senior Consulting Engineer for expert system manufacturer Gensym Corporation and 10 years as Lead Engineer, Intelligent Systems for General Atomics (GA), where he led GA in the development of reusable Prognostics and Health Management systems applied to various industries. He founded D2K Technologies in 2014, a solution provider of intelligent modelbased reasoning systems for mission critical systems. He also serves as a PHM subject matter expert for NASA, with active projects at SSC and JSC. He resides with his family in Oceanside, California. 


\section{Industry Forum:}

Moving from: "React and Repair" to "Predict and Prevent"

May $8-11,2018$

\section{Communication \& Information Flow to support PHM - Presentations \&}

\section{Panel}

\section{Will Sobel, Vimana}

Presentation Title: Health and Maintenance Through the Lens of Dynamic Scheduling

Bio: Mr. Will Sobel is Chief Strategy Officer and Co-Founder of VIMANA, the leading analytics platform for discrete manufacturing, and the Principle Architect and Chair of the Technical Steering Committee for the MTConnect Standard, the leading international semantic standard for manufacturing equipment. In addition, he is also the Co-Chair of the Industrial Artificial Intelligence (AI) Task Group at the Industrial Internet Consortium.

Mr. Sobel brings over 30 years of experience in software architecture and is currently advancing VIMANA, as well as researching standards-based solutions for self-aware Industrial Internet of Things (IloT) systems in manufacturing. Prior to co-founding System Insights, Mr. Sobel was a visiting lecturer at UC Berkeley and worked for many years developing distributed and SaaS analytics applications financial industry.

Moneer Helu, Systems Integration Division, NIST

Presentation Title: Connecting and Deploying Smart Manufacturing Technology to Support PHM

Bio: Moneer Helu is the Leader of the Life Cycle Engineering Group in the Systems Integration Division of the Engineering Laboratory at NIST. He co-leads the Prognostics, Health Management, and Control project in the Smart Manufacturing Operations Planning and Control program as well as the NIST Smart Manufacturing Systems Test Bed. Dr. Helu's current research focuses on developing the digital thread to enable and support diagnostics, prognostics, and control for smart manufacturing systems on the shop floor. He has also made contributions in the areas of green manufacturing, process monitoring, and manufacturing data interoperability and management. Dr. Helu is a member of the Technical Steering Committee and Technical Advisory Group for MTConnect, Executive Committee of the ASME Manufacturing Engineering Division, and a Corporate Member of the International Academy for Production Engineering (CIRP).

\section{Joel Neidig, ITAMCO}

\section{Presentation Title: Starting Small and Scaling MTConnect Across Multiple Factories}

Bio: Joel Neidig has a bachelor's degree from Bob Jones University in operations management and has had 13 years of experience integrating manufacturing technology and software development. Neidig sits on the Technical Advisory Group for MTConnect, an open-source, royalty-free standard that is intended to foster greater interoperability between devices and software applications. He has been an active member of MTConnect since 2009. Neidig developed the first iOS and Android-compatible MTConnect apps, and has developed over 65 manufacturing apps for the App Store and Google Play, which have been downloaded over half a million times. Neidig also sits on the Technical Advisory Committee for the Digital Manufacturing and Design Innovation Institute, a federallyfunded research and development organization, that encourages factories across America to deploy digital manufacturing and design technologies, so those factories can become 


\section{Industry Forum:}

Moving from: "React and Repair" to "Predict and Prevent" May $8-11,2018$

more efficient and cost-competitive. His company was voted the 2014 Autodesk Inventor of the Year by the Autodesk Community. He has been named as a manufacturing "Thought Leader" by IMTS Insider. Neidig recently won second place in the MTConnect Challenge at the 2014 MC2 Conference for his application: "Expanding Manufacturing's Vision: MTConnect + Google Glass," sponsored by the National Center for Defense Manufacturing and Machining and the Office of the Secretary of Defense. Since the founding of Manufacturing USA, Neidig and his company have been involved in 4 research projects with DMDII and 2 research projects with America Makes, one of which has transitioned to an additive manufacturing tech startup founded by his company called Atlas 3D and has hired 4 employees as the result of the research they have done with Manufacturing USA. He has previously been the manufacturing keynote speaker at Autodesk University and recently presented at the Automotive Innovation Forum. Neidig was the recipient of SME's 2015 Outstanding Young Manufacturing Engineer of the Year Award (the award is named in honor of a specific individual who has made lifelong contributions to manufacturing and recognizes exceptional contributions and accomplishments in the manufacturing industry) and was also named one of the 30 Advanced Manufacturing Visionaries by Smart Manufacturing Magazine. He was also the recipient of the 2016 AGMA Next Generation Award which recognizes his contributions and leadership to the members of the American Gear Manufacturers Association and the gear industry. His company was also awarded a Phase I SBIR grant from DARPA for Secure Messaging on the Blockchain Architecture. Recently, Joel spoke at the White House by invitation of the National Economic Council in recognition of the progress that has been made by himself and his company in the Manufacturing USA program. Neidig is very involved in an advisory role at the ITAMCO Manufacturing Education Center located at Plymouth High School, which was started by his company to prepare students for the challenges of careers in manufacturing.

Rob Andes, The Knowledge Design Company

Presentation Title: Asset Life-Cycle Information Management (ALCIM) Technologies for Prognostic Analysis of CNC Machines

Bio: Rob Andes is Principal Engineer - Systems Engineering - for The Design Knowledge Company (TDKC) out of Fairborn, $\mathrm{OH}$, located near Wright-Patterson Air Force Base. He is Principal Investigator on multiple R\&D efforts, including development of early concepts to fielding of deployed applications for the US Air Force, US Navy and industry. In addition, he is commercialization lead for TDKC high-technology software products. He has a wide range of experience in analysis and design of intelligent user interfaces in complex humanmachine domains with focus in development of C3 systems for mission critical support systems utilizing Al for adaptive aiding systems in the space, aviation, and manufacturing domains. As a technology leader and innovator, he has held positions as a key executive and lead team member of R\&D efforts and software applications companies, from successful start-up CTO to turn-around specialist at a Fortune 100 company.

Currently, Andes is Principal Investigator on US Air Force sponsored programs for the development of decision aiding applications and condition-based maintenance (CBM) technologies for CNC machine prognostics using analytics and machine-learning techniques. 


\section{Industry Forum:}

Moving from: "React and Repair" to "Predict and Prevent"

May $8-11,2018$

The Costs and Benefits of Advanced Maintenance in Manufacturing

Doug Thomas, Applied Economics Office, NIST

Presentation Title: The Costs \& Benefits of Advanced Maintenance in Manufacturing

Bio: Douglas S. Thomas is a research economist for the Engineering Laboratory's Applied Economics Office at NIST. Currently, his activities are focused in two areas of research: 1) manufacturing industry costs and resource consumption and 2) methods for economic decision making in the adoption of technologies and processes in manufacturing. The first area includes measuring and tracking the U.S. manufacturing supply chain using methods such as economic input-output analysis. The second area of research studies barriers to technology and process adoption in manufacturing as well as identifies methods for economic decision making in the adoption of technologies and processes. 


\section{Industry Forum:}

Moving from: "React and Repair" to "Predict and Prevent"

May $8-11,2018$

\section{Wednesday, May 9, 2018}

\section{PLENARY - NIST Smart Manufacturing Programs: Driving Innovation and Reducing Risks of Adoption of New Technologies}

Al Wavering, Intelligent Systems Division, NIST

Bio: Albert J. Wavering is Chief of the Intelligent Systems Division (ISD) of the Engineering Laboratory at NIST. ISD develops measurement science solutions for intelligent systems technologies to help its manufacturing industry and government customers drive innovation and enhance their competitiveness and mission effectiveness. Wavering has been at NIST since 1985, serving in a variety of technical and management roles, including mechanical engineer, group leader, program manager, and program analyst in the NIST Director's Office prior to his current position. He also served for three years as the Acting Deputy Director of the NIST Manufacturing Engineering Laboratory. His research background includes work in robotics and automation, sensing and control, and manufacturing production equipment. He is a Fellow of the Society of Manufacturing Engineers.

\section{Emerging Sensing Technologies to Enable Monitoring, Diagnostics, and Prognostics - Presentations \& Panel}

Radu Pavel, TechSolve

Presentation Title: An MTConnect ${ }^{\circledR}$-based Approach for Machine Health Monitoring

Bio: Dr. Radu Pavel is Vice President of Engineering and Chief Technology Officer of TechSolve, Inc., a process improvement and machining services organization located in Cincinnati, OH, U.S.A. Dr. Pavel has over 20 years of experience in industry and research laboratories from Europe and United States. He has a Master of Science in Mechanical Engineering, and two PhDs - one in Mechanical Engineering and one in Manufacturing Engineering.

Dr. Pavel's core expertise includes machining and grinding processes, monitoring of machining equipment and processes, modeling and simulation, test-bed development and instrumentation, data acquisition and analysis, and teaching and training. Dr. Pavel has conducted research and development with Smart Manufacturing technologies for over 12 years. He has been involved with technologies specifically focused on machine health and maintenance since 2007.

Dr. Pavel has published multiple papers in refereed conference proceedings and journals, and organized symposia focused on digital manufacturing, smart machine technologies, and advances in material processing and inspection. He is currently Associate Editor for the Journal of Manufacturing Science and Engineering, and a member of the Executive Committee of Manufacturing Engineering Division of ASME. 


\section{Industry Forum:}

Moving from: "React and Repair" to "Predict and Prevent"

May $8-11,2018$

Brittany Newell, Purdue University

Presentation Title: Capacitance Sensors for Industrial Applications

Bio: Dr. Brittany Newell is an assistant professor at Purdue University in the Purdue Polytechnic Institute School of Engineering Technology. Brittany received her B.S. in Biomedical Engineering from Purdue University and her M.S. and Ph.D. in Agricultural and Biological Engineering from Purdue University. She then worked in industry as a Quality Manager for a contract manufacturing company before joining the Purdue faculty. Brittany completed her Ph.D. in the field of electroactive polymers for industrial applications. Her current research interests are focused on adaptive structures, energy transduction, and methods of manufacturing these materials. She focuses on additive manufacturing techniques for material sensors and actuators and their characterization and production.

\section{Justinian Rosca, Siemens Corporation}

Presentation Title: Validation of the Intelligent Edge

Bio: Justinian Rosca is Senior Key Expert of Siemens Corp., Corporate Technology in Princeton NJ. He holds Ph.D. and M.Sc. degrees in Computer Science from the University of Rochester and a Dipl. Eng. Degree in Computer and Control Engineering from the Polytechnic University Bucharest. Dr. Rosca is presently an Affiliate Researcher at Princeton University, Electrical Engineering Department and was an Affiliate Professor at the University of Washington, Electrical Engineering Department, from 2008 to 2011. He obtained a certificate in executive management for innovation, from the University of Pennsylvania, Wharton School of Business. Dr. Rosca's primary research interests span statistical signal processing, machine learning, probabilistic inference, artificial intelligence, sensing and communication, with an emphasis on embedded intelligence in autonomous systems and cyber physical systems. Dr. Rosca holds over 50 patents, 100 publications in the areas of signal processing, machine learning, communications, cyber-physical systems, and co-authored two books in mathematics and signal processing. His scientific contributions were transferred into a variety of products and systems representing embedded intelligence in systems such as microphone array technologies for hearing aids and mobile phones, adaptive multimedia wireless network management, connected and autonomous vehicles, and run-time edge intelligence in industry. These contributions earned him multiple Siemens business unit awards. He served as program chair of the 6th Independent Component Analysis and Blind Signal Separation International Conference, chair of the Neural Information and Processing Systems workshop on Sparse Representations in Signal Processing, and recently as chair of the Data Challenge 2015, 2016 and 2017 competitions of the Prognostics and Health Management (PHM) Society.

Greg VogI, Intelligent Systems Division, NIST

Presentation Title: Emerging Sensing Technologies Towards Smart Machine Tools

Bio: Greg Vogl is a Mechanical Engineer at the National Institute of Standards and Technology (NIST) in Gaithersburg, Maryland. After working with MEMS as a National Research Council Postdoctoral Researcher, he joined NIST and worked on machine tool metrology and standards development. Currently, Greg is a member of the Prognostics, 


\section{Industry Forum:}

Moving from: "React and Repair" to "Predict and Prevent"

May $8-11,2018$

Health Management, and Control (PHMC) project, which seeks to enable robust real-time diagnostics and prognostics for smart manufacturing systems. Specifically, Greg works on solutions to transform machine tools into smart machine tools.

\section{Ed Spence, Machine Instrumentation}

Presentation Title: Machine Health for the Machine Maker

Bio: Ed Spence is the Managing Director and Founder of The Machine Instrumentation Group, a collaborative network of CBM product and service providers helping machine OEMs to instrument their own equipment. Prior to that, Ed was the Marketing Manager for the MEMS Sensor Technology Group at Analog Devices, where he defined the accelerometer roadmap for Condition Monitoring.

\section{Planning and Assessment to Promote Monitoring, Diagnostic, and Prognostic Technologies - Presentations \& Panel}

Karl Reichard, Penn State University Applied Research Lab

Presentation Title: Driving Requirements For Prognostics - How Far In The Future Do We Need to Predict?

Bio: Karl M. Reichard, Ph.D., heads the Applied Research Laboratory (ARL) Embedded Hardware/Software Systems and Applications Department. Dr. Reichard has over 25 years of experience in the design and development of advanced measurement, control, and monitoring systems. He received Ph.D., M.S., and B.S. degrees in Electrical Engineering from the Virginia Polytechnic Institute and State University (Virginia Tech). Dr. Reichard is a Research Associate with the Pennsylvania State University ARL, and an Assistant Professor of Acoustics with the Penn State Graduate Program in Acoustics. He leads a group at the ARL focused on the development of embedded sensing and processing hardware and software systems. His own research experience includes the development of embedded and distributed sensing and control systems for robotics, noise cancelation, acoustic surveillance and detection, machinery and system health monitoring, and electro-optics. Dr. Reichard is a member of the Board of Directors of the Prognostics and Health Management Society, the IEEE, and the Acoustical Society of America. He is the author of over 50 papers and articles published in journals and conference proceedings. 


\section{Industry Forum:}

Moving from: "React and Repair" to "Predict and Prevent"

May $8-11,2018$

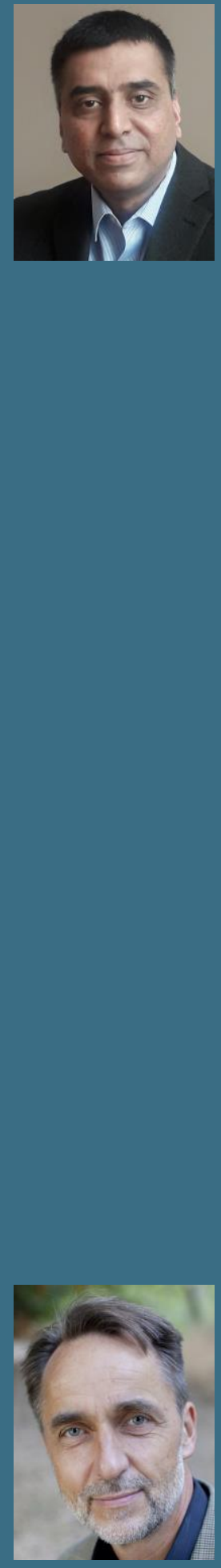

Ananth Seshan, MESA International

Presentation Title: Learnings from Use Cases on Proactive Asset Performance Management Bio: Dr. Ananth Seshan is the Chairman of 5G Technologies Ltd., a digital automation solutions group. The group is headquartered in Ottawa and has operations in Mexico and India. He has been the main thought leader behind the successful flagship product of the group, Enterprise Gateway. Enterprise Gateway has a user footprint in 20 countries globally and more than 100 installations in large manufacturing organizations and utilities. The product is the first of its kind in achieving vertical integration between production and the enterprise operations and has served in the field for more than 750,000 hours in major global manufacturing and utility companies.

Ananth has been a consultant to several large manufacturing organizations and utilities worldwide for the last 3 decades in the areas of robotics, automation, and of late, Asset Performance Management and Digital Manufacturing. He was an invited member of a Special Task Force set up by Industry Canada during the turn of the millennium to explore the viability of applying advanced manufacturing technologies as a strategy to build competitive advantage in the manufacturing segment in Canada. He has also served as an invited member of the Steering Committee of a Canadian Federal Center of Excellence in Robotics and Automation. He has been an invited speaker in many international forums on manufacturing automation and has won several awards for innovation and entrepreneurship. He is presently the Chairman of the Asset Performance Management Working Group of MESA, a leading not for profit organization in North America that establishes standards and best practices for the manufacturing industry. Ananth has several publications in journals and international conference proceedings, owns 2 patents, and has authored a chapter in a book published by Springer in 2015 on "Innovative Process Development in the Metallurgical Industry."

Ananth completed his undergraduate degree in Production Engineering from the Madras University and a Masters Degree in Robotics and Automation from Indian Institute of Technology, Chennai. He received his Ph.D. from the University of Toronto in 1992 in the area of "Common-Sense Reasoning" in Robotic Mechanisms.

Kai Goebel, National Aeronautics and Space Administration (NASA)

Presentation Title: Designing Resilient Engineered Systems with Prognostics and Health Management

Bio: Kai Goebel is the Tech Area Lead for Discovery and Systems Health at NASA Ames Research Center which covers the areas of real time monitoring for resilience, safety, diagnostics, and prognostics applied to aeronautics and space systems. Dr. Goebel received a Ph.D. from the University of California at Berkeley in 1996 with a dissertation on monitoring for manufacturing systems. Between 1997 and 2006, he worked at General Electric's Corporate Research Center in upstate New York where he developed techniques for a number of GE's industrial applications such as aircraft engines, terrestrial transportation systems, energy applications, and medical systems. He was an adjunct professor at Rensselaer Polytechnic Institute where he taught courses in Applied Al. Dr. 


\section{Industry Forum:}

\section{Moving from: "React and Repair" to "Predict and Prevent"}

May $8-11,2018$

Goebel is now an adjunct professor at Lulea Technical University. He has been co-adviser to a dozen Ph.D. students. He is a co-founder of the Prognostics and Health Management Society and he is currently associate editor of the International Journal of PHM. He holds 18 patents and has published 300 papers in the field. He is member of the SAE Health Management steering committee and the IVHM working group.

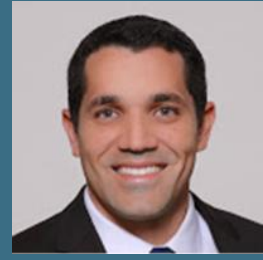

Miguel Sáez, University of Michigan

Presentation Title: Modeling and Analysis of Cyber-Physical Manufacturing Systems for Anomaly Detection

Bio: Miguel Sáez received his Mechanical Engineering degree from La Universidad del Zulia, Venezuela in 2008, the M.Eng in Global Automotive and Manufacturing from the University of Michigan, USA in 2015 and is now working towards the Ph.D. in Mechanical Engineering developing innovative ideas to improve productivity and responsiveness of automated manufacturing systems.

He worked as a Product Engineer at Dana Holding Corporation, Venezuela from 2007 to 2010, leading multidisciplinary design and manufacturing projects, coordinating cost-saving projects using CAD/CAE tools, and testing NVH for vehicle powertrains. He worked as a Senior Manufacturing Engineer at General Motors, Venezuela from 2010 to 2013, developing, testing, and installing semi-automated manufacturing systems, and managing a capital investment project for new vehicle programs.

\section{Jorge Arinez, General Motors}

Presentation Title: Towards Systems Integrated Production and Maintenance Operations

Bio: Dr. Jorge Arinez is a Group Manager in the Manufacturing Systems Research Lab at GM Global Research and Development. His main responsibilities involve strategically defining and managing portfolios of advanced manufacturing systems projects. This also includes leading their development and implementation throughout GM's global manufacturing operations. Recently, his research is focused on the development of analytical tools for real-time production and process control, maintenance, and asset optimization with a focus on energy efficiency and sustainability of manufacturing systems. 


\section{Industry Forum:}

Moving from: "React and Repair" to "Predict and Prevent"

May $8-11,2018$

\section{Monitoring and Analysis Technologies for Prognostics and Health Management (PHM) - Presentations \& Panel \\ David Siegel, Predictronics}

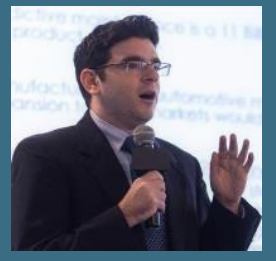

Presentation Title: Perspectives and Case Studies on PHM Technologies for Manufacturing

Bio: Dr. David Siegel is currently the Chief Technology Officer for Predictronics Corp. His current role includes developing the technology road map for the company's predictive monitoring software and service solutions, developing new algorithms and methodologies, as well as leading a data science team to carry out the customization and deployment of various predictive monitoring solutions. Dr. Siegel has led numerous efforts on diagnostic and prognostic software for a variety of industrial customers and applications. A sample of these efforts include advanced diagnostic methods for industrial robots, health monitoring systems for railway applications, failure prediction tools for machine tool bearings, and intelligent maintenance systems for military ground vehicles. Dr. Siegel is also a two-time winner of the Prognostics and Health Management Data Challenge and has won several best paper awards at various conferences focused on predictive monitoring and data analytics.

\section{Nancy Diaz-Elsayed, University of South Florida}

Presentation Title: Production Monitoring for Performance and Energy Efficiency Improvements

Bio: Dr. Nancy Diaz-Elsayed is a Research Assistant Professor at the University of South Florida (USF) in the Department of Civil and Environmental Engineering. She obtained her Ph.D. in Mechanical Engineering from UC Berkeley and prior to working at USF, she was the Sustainable Manufacturing Specialist at Autodesk. Her projects have spanned discrete and continuous processes, including the development of a building intelligence application that combined real-time data with Building Information Models to improve the performance of factories and commercial buildings, and the sustainable design of integrated water and wastewater treatment systems. Her research interests include the modeling of complex systems and processes, technology development for smart and sustainable manufacturing, and the role of industrial symbiosis in the design and growth of urban environments.

\section{Sanket Amberkar, Falkonry}

Presentation Title: Predictive Analytics Approach with Time Series Data using Machine Learning

Bio: Sanket leads marketing at Falkonry and is responsible for the company's positioning, thought leadership and go to market strategy. Sanket is the SVP of Marketing at Falkonry and has over 20 years of experience in the high tech, energy, industrial and automotive markets in areas ranging from of product development to market strategy. Prior to Falkonry, he was VP of Product Marketing for Innovation \& New Ventures at Flex, where he brought to market its Innovation services and launched the LabIX startup initiative. Earlier, he led marketing and product development teams at Cisco and Delphi. Sanket holds Master's degrees in Electrical Engineering and Business Administration - both from the University of Michigan. He is a frequent industry speaker and holds thirteen U.S. patents. 


\section{Industry Forum:}

Moving from: "React and Repair" to "Predict and Prevent"

May $8-11,2018$

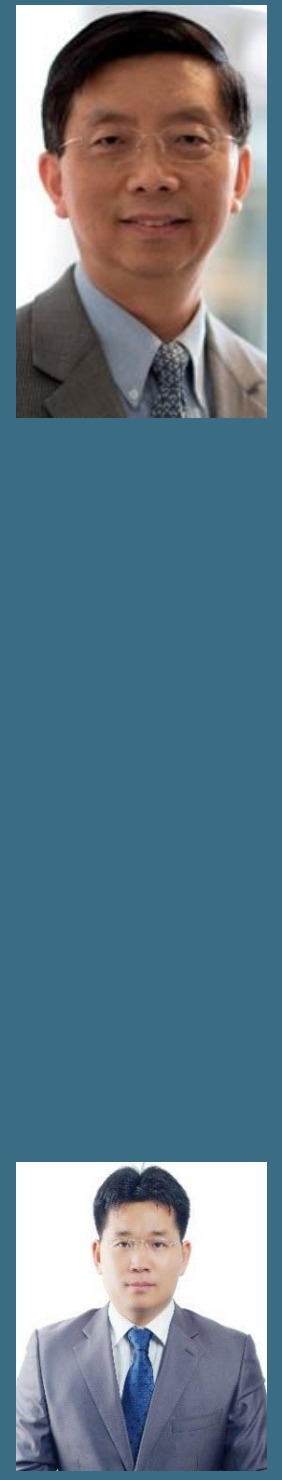

Robert Gao, Case Western Reserve University

Presentation Title: Stochastic Modeling for System Remaining Life Prognosis

Bio: Robert Gao is the Cady Staley Professor of Engineering and Chair of the Mechanical and Aero-space Engineering department at Case Western Reserve University in Cleveland, Ohio. Since receiving his Ph.D. degree in 1991 from the Technical University of Berlin, Germany, he has been working on multi-physics sensing methodologies, design, modeling, and characterization of measurement systems, multi-resolution signal analysis, and energyefficient sensor networks for improving the observability of dynamical systems such as manufacturing machines and enhancing manufacturing process and product quality control.

Prof. Gao is a Fellow of the ASME, IEEE, SME, and CIRP (International Academy for Production Engineering), and an elected member of the Connecticut Academy of Science and Engineering. He was a Distinguished Lecturer of the IEEE Instrumentation and Measurement Society and IEEE Electron Devices Society. He served as a Guest Editor for the Special Issue on Data Science-Enhanced Manufacturing of the ASME Journal of Manufacturing Science and Engineering, and was an Associate Editor for the ASME Journal of Dynamical Systems, Measurement, and Control, IEEE Transactions on Instrumentation and Measurement, and IFAC Journal of Mechatronics. He is a recipient of the ASME Blackall Machine Tool and Gage Award, IEEE Instrumentation and Measurement Society's Technical Award, multiple Best Paper awards, Outstanding Junior and Senior Faculty awards, Outstanding Research Award, and an NSF CAREER award.

\section{ChaBum Lee, Tennessee Tech University}

Presentation Title: On-Machine Dimensional Measurement Technology for Prognostics and Health Monitoring for Precision Manufacturing Systems and Processes

Bio: Dr. Lee is currently an Assistant Professor within the Department of Mechanical Engineering at Tennessee Tech. University where he's been since 2015. He will be an incoming Assistant Professor in the Department of Mechanical Engineering at Texas A\&M University beginning this fall. Prior to his time at Tennessee Tech. he was a Research Assistant Professor (2014-2015) - Department of Mechanical Engineering, University of South Carolina and a Research Associate (2013-2014) - Department of Mechanical Engineering, University of South Carolina. Prior to his university positions, he was a Senior Researcher (2010-2013) at LG Display Co. Ltd. in Paju, Korea. Dr. Lee earned his Ph.D. in 2012 in Mechatronics at Gwangju Institute of Science and Technology (Korea). 


\section{Industry Forum:}

Moving from: "React and Repair" to "Predict and Prevent"

May $8-11,2018$

\section{Emerging Research Efforts}

Junmin Lee, Seoul National University (SNU)

Presentation Title: Exercising Standardization of Prognostics and Health Management (PHM) for Manufacturing Industry

Bio: Junmin Lee received the B.S. degree with a double major in Biosystems Engineering and Mechanical Engineering from Seoul National University, Seoul, Republic of Korea, in 2013. He is currently pursuing the Ph.D. degree at the Department of Mechanical and Aerospace Engineering in Seoul National University, Seoul, Republic of Korea. His current research topics include prognostics and health management for electronic products and electric machine drive systems. He was the winner in PHM Society Data Challenge Competition in 2017.

\section{Chan Hee Park, SNU}

Presentation Title: Fault Detection of an OHT (Overhead Hoist Transport) Vehicle Using Feedback Control Signals

Bio: Chan Hee Park received her B.S. degree from Seoul National University, Seoul, Republic of Korea, in 2016. She is currently pursuing the Ph.D. degree at the Department of Mechanical and Aerospace Engineering in Seoul National University, Seoul, Republic of Korea. Ms. Park's research topics include prognostics and health management (PHM) for electric machines using a data-driven approach. Ms. Park was the winner in PHM Society Data Challenge Competition in 2017 and received the Korean Society of Mechanical Engineers (KSME)-SEMES Innovation Challenge Award in 2017.

\section{Using Unstructured Work Order Data to Improve Maintenance Procedures in Manufacturing}

Bio: Michael P. Brundage, Ph.D. is an Industrial Engineer in the Informational Modeling and Testing Group at NIST. Dr. Brundage's interests include Smart Manufacturing Diagnostics for Intelligent Maintenance, Sustainable Manufacturing Performance Measurement, Smart Manufacturing Capability Assessment, and Manufacturing Knowledge Visualization. His work contributes to guidelines for intelligent maintenance and he is part of a task group for creating an ASME Prognostics Health Management (PHM) standards committee. He also worked closely with ASTM International E60.13 in the development of a guideline for sustainable manufacturing performance indicators (ASTM E3096-17). He authored over 25 peer reviewed publications and has chaired multiple ASME MSEC Symposia and industry forums/workshops at NIST. Dr. Brundage is the recipient of the 2018 ASME Old Guard Early Career Award and was selected as one of SME's 2018 Class of 30 Under 30. 


\section{Industry Forum:}

Moving from: "React and Repair" to "Predict and Prevent"

May $8-11,2018$

\section{NIST Research on Monitoring, Diagnostics, and Prognostics for}

\section{Manufacturing Workcells}

Brian A. Weiss, Intelligent Systems Division, NIST

Bio: Dr. Brian A. Weiss is a mechanical engineer and the project leader of the Prognostics, Health Management, and Control (PHMC) project within the Engineering Laboratory (EL) at NIST. His current research efforts are focused on developing the necessary measurement science to verify and validate emerging monitoring, diagnostic, and prognostic technologies and strategies for smart manufacturing to enable manufacturers to respond to planned and un-planned performance changes. The project is focused on the application domains of machine tools and robot systems. From 2013-2016, Dr. Weiss also served as the Associate Program Manager for the Smart Manufacturing Operations Planning and Control (SMOPAC) program which contains his PHMC project. Prior to his manufacturing research, he spent 15 years conducting performance assessments across numerous military and first response technologies including autonomous unmanned ground vehicles; tactical applications operating on Android ${ }^{\mathrm{TM}}$ devices; advanced soldier sensor technologies; free-form, two-way, speech-to-speech translation devices for tactical use; urban search and rescue robots; and bomb disposal robots. He also spent six years developing robotic crane technologies which included the deployment of a prototype system on a military installation. Dr. Weiss is a current member of the PHM Society Board of Directors and serving on an ASME task group aimed at building up PHM standards and guidelines. His efforts have earned him numerous awards including a Government Computer News (GCN) for IT Excellence Award (2014), Department of Commerce (DOC) Gold Medal (2013), Colleague's Choice Award (2013), DOC Silver Medal (2011), DOC Bronze Medals (2004 \& 2008), and the Jacob Rabinow Applied Research Award (2006). He earned two Best Paper and Best Presentations awards from the International Test and Evaluation Association (ITEA). He has a B.S. in Mechanical Engineering (2000), Professional Masters in Engineering (2003), and Ph.D. in Mechanical Engineering (2012) from the University of Maryland, College Park, Maryland, USA. 


\section{Industry Forum:}

Moving from: "React and Repair" to "Predict and Prevent"

May $8-11,2018$

\section{Thursday, May 10, 2018}

\section{PLENARY - CCAM Activities in Highly Scalable Computing Systems to} Monitor and Analyze Industrial Processes, Diagnose Irregularities, and Automatically Adapt Machine and Process Behaviors

Jaime Camelio, Commonwealth Center for Advanced Manufacturing and Virginia Tech University

Bio: Dr. Jaime Camelio is currently the Chief Technology Officer at the Commonwealth Center for Advanced Manufacturing (CCAM) and the Rolls-Royce Commonwealth Professor for Advanced Manufacturing in the Grado Department of Industrial and Systems Engineering at Virginia Tech. Dr. Camelio obtained his B.S. and M.S. in Mechanical Engineering from the Catholic University of Chile in 1994 and 1995, respectively. In 2002, he received his Ph.D. from the University of Michigan.

\section{Standards and Best Practices - Presentations \& Panel}

Andrew Hess, Hess PHM Group

Presentation Title: PHM - A Key Element Across the Continuum of a Digital Enterprise

Bio: Andy brings program management, technical, and engineering, logistics, and asset management expertise as a globally recognized leader and expert in the fields of diagnostics and predictive maintenance. For over 35 years, at the Naval Air System Command, Andy led the innovation, development, and implementation of condition monitoring systems for all the Navy fixed wing and helicopter applications. He is widely recognized as a leader in the area of jet engine monitoring systems. Andy helped formulate the autonomic logistics information system concept. Andy is a widely-used consultant to industry, government, and academic organizations in the fields of advanced diagnostics, prognostics, health and asset management, and enterprise-wide applications. Andy is the current president of the PHM Society and remains active in many other professional, advisory, and standards organizations and committees. Andy is also a recent Lifetime Achievement Award recipient from the PHM Society.

Andy started his career in flight testing at the Naval Air Test Center and Naval Air Warfare Center evaluating aircraft systems; developing the first comprehensive engine monitoring system; and playing significant roles in the development of military aircraft. He has been a Senior Engineering Fellow and a Fellow of the Society for Integrated Engineering Asset Management. He led the PHM effort for the Joint Strike Fighter JPO. Through his consulting firm, Andy helped DARPA structure and manage their large Prognosis program. Some of his other clients have included: Bell Helicopter, Boeing, General Atomics, NASA, Honeywell, the US Army CECOM, Sikorsky, Teledyne Controls, the Australian and Canadian governments, the University of Maryland CALCE, and sundry small businesses. 


\section{Industry Forum:}

Moving from: "React and Repair" to "Predict and Prevent"

May $8-11,2018$

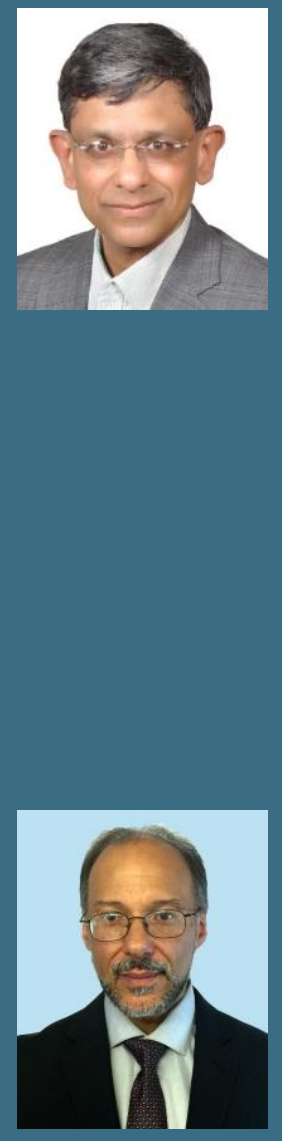

Ravi Rajamani, $d r R 2$

Presentation Title: The Role of Standards in Simplifying the Job of Engineering Complex Products

Bio: Dr. Ravi Rajamani is an independent consultant who has accumulated years of experience in aerospace propulsion and energy, specifically in data analytics and modelbased methods for controls, diagnostics, and prognostics. He has many publications including three books (chief being Electric Flight Technology: The Unfolding of a New Future), book chapters, journal and conference papers, and patents. Prior to his current job, Ravi worked at Meggitt, United Technologies Corporation, and the General Electric Company. He has a BTech from IITD, an MS from IISc, a PhD from University of Minnesota, and an MBA from University of Connecticut. He is active within various SAE technical committees dealing with PHM. He is also active in the PHM Society, serving on its board of directors. Ravi is a Visiting Professor of Aerospace, Transport and Manufacturing at Cranfield University. He is the editor-in-chief of the SAE International Aerospace Journal; has been elected a fellow of SAE; and is a recipient of its Forest R. McFarland Award.

\section{Tom Fiske, Yokogawa}

\section{Presentation Title: ISA 108 Intelligent Device Management}

Bio: Dr. Tom Fiske, Principal Technology Strategist, is part of Yokogawa's Global Strategic Technology Center. He is responsible for establishing the vision of Yokogawa's Advanced Decision Support solutions that help improve operators' situational awareness and effectiveness and contributes to Yokogawa's overall automation strategy. Dr. Fiske has more than 30 years of hands-on experience in research, product development, project management, and process engineering. Throughout his career, he has actively been involved in simulating and optimizing complex production processes. Dr. Fiske has consulted with end-users to address key issues concerning selection, adoption, implementation, and use of manufacturing, automation and control, and production and engineering technology.

Dr. Fiske is an active member in numerous Standards Development Organizations, including ISA, ANSI, and IEC. Dr. Fiske is a graduate of Stevens Institute of Technology with a Ph.D. in Chemical Engineering. He also holds a Master of Science in the Management of Technology from the Sloan School at Massachusetts Institute of Technology (MIT).

\section{Tom Hedberg, Systems Integration Division, NIST}

Presentation Title: Practice and Specification Standards for Design and Manufacturing Bio: Thomas Hedberg, Jr. is a Mechanical Engineer in the Systems Integration Division of the Engineering Laboratory at NIST. He is the Project Leader of the Digital Thread for Smart Manufacturing project in the NIST Smart Manufacturing Operations Planning and Control program and the Co-Leader of the NIST Smart Manufacturing Systems Test Bed. His current research focus is in the areas of digital-product design, smart manufacturing, and lifecycle engineering. Mr. Hedberg is a Voting Member of the American Society of Mechanical Engineers (ASME) Y14.37, Y14.41, and Y14.41.1 subcommittees from the ASME Y14 suite of standards and Co-Chair and Americas Lead for the Visualization Working Group for LOTAR International. 


\section{Industry Forum:}

Moving from: "React and Repair" to "Predict and Prevent"

May $8-11,2018$

Prior to joining NIST, Mr. Hedberg was a Senior Mechanical Engineer and Technical Lead of the Model Based Enterprise (MBE) group at Honeywell Aerospace. In this role, he developed a strategy and implementation of MBE in Honeywell's engineering operations. He earned a M.Eng. in Engineering Management with a concentration on Systems Engineering from the Penn State University and a B.S. in Aeronautical and Astronautical Engineering from Purdue University. He is currently a Ph.D. Candidate in Industrial and Systems Engineering at the Virginia Polytechnic Institute and State University. Mr. Hedberg is a licensed Professional Engineer (PE) in the States of Arizona and Maryland.

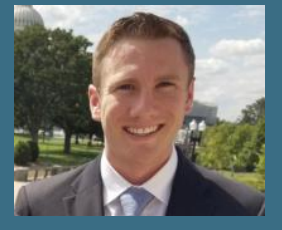

Logen Johnson, SAE

\section{Presentation Title: Best Practices in Developing PHM Standards}

Bio: Logen Johnson has been with SAE International for 2 years and is based in Washington, DC. In this role, Logen is responsible for supporting standards development operations for SAE's aerospace standards program. This includes working with the U.S. and global aerospace community on new standards development as well as global strategy and outreach for SAE.

Prior to joining SAE, Logen worked with other standard organizations in DC. He holds a BS degree from Wentworth Institute of Technology in Electromechanical Engineering.

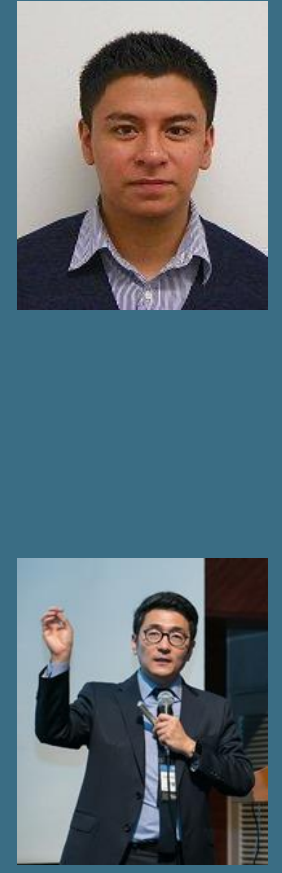

\section{Donnie Alonzo, $A S M E$}

\section{Presentation Title: ASME Manufacturing Standards Overview}

Bio: Donnie Alonzo earned a Bachelor of Science degree in mechanical engineering from Columbia University in the City of New York. He has since been a Standards and Certification Engineer for the American Society of Mechanical Engineers. Now working with the power and energy related standards committees, he has previously worked with numerous other standards development committees related to manufacturing, and has been helping lead the effort for ASME guidelines in monitoring, diagnostics, and prognostics.

\section{PHM within the International Manufacturing Community - Presentations}

\section{Byeng Youn, SNU}

Presentation Title: Frontiers in Korean Manufacturing Prognostics - Success Episodes and Issues

Bio: Prof. Byeng D. Youn is the Professor of Mechanical and Aerospace Engineering at Seoul National University (SNU) and the CEO of OnePredict Inc. (onepredict.com). Before joining SNU, he was an Assistant Professor in the Department of Mechanical Engineering at the University of Maryland, College Park. He is currently the Future-Tech Consulting Fellow of LG Electronics. He earned the Ph.D. degree from the University of lowa in 2001. His research goal is to develop rational reliability and design methods based on mathematics, physics, and statistics for use in complex engineered systems, mainly focused on energy systems. His current research includes reliability-based design, prognostics and health management (PHM), energy harvester design, and statistical verification and validation (V\&V). His dedication and efforts in research have garnered substantive peer recognition 


\section{Industry Forum:}

Moving from: "React and Repair" to "Predict and Prevent"

May $8-11,2018$

resulting in notable awards including the ISSMO/Springer Prize for a Young Scientist (2005), the Young Faculty Development Award from the U.S. Nuclear Regulatory Commission (2009), the IEEE PHM Competition Winner (2014), the PHM Society Data Challenge Winners (2014, 2015, 2017), the Shinyang Academic Award (2017), and the ASME IDETC Best Paper Awards $(2001,2008)$. He has over 300 publications (85 journal articles, over 250 international conference proceedings, and four book chapters) in the area of reliability analysis and design, energy harvesting, and PHM. He also serves as an Editor of many notable journals including Structural and Multidisciplinary Optimization (SMO), International Journal of Precision Engineering and Manufacturing (IJPEM), Journal of Mechanical Science and Technology (JMST), and JMST Advances. His research has been supported by the National Research Foundation (NRF) in Korea, Korea Electric Power Corporation (KEPCO), Samsung Electronics, U.S. Army, Hyundai Motors, LG Electronics, General Motors, and his accumulated funds amounting to 10 million dollars.

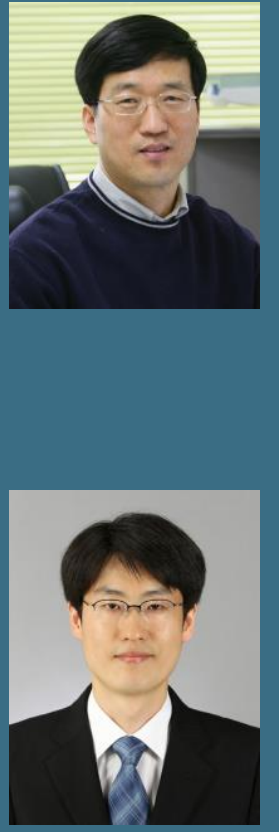

\section{Hyunbo Cho, Pohang University of Science and Technology (POSTECH)}

Presentation Title: Data-driven Prognostics for an Assembly Machine for Automatic Transmissions

Bio: Hyunbo Cho is a professor of Department of Industrial and Management Engineering at POSTECH. He received his BS and MS degrees in Industrial Engineering from Seoul National University in 1986 and 1988, respectively, and his PhD in Industrial Engineering with a specialization in Manufacturing Systems Engineering from Texas A\&M University in 1993. His areas of expertise include Smart Manufacturing Systems, Big Data and Predictive Analytics and Cyber-Physical Production Systems.

\section{Hyunseok Oh, Gwangju Institute of Science and Technology (GIST)}

Presentation Title: Korea's Efforts towards PHM in Semiconductor and Automotive Manufacturing

Bio: Hyunseok Oh is an Assistant Professor with the School of the Mechanical Engineering, Gwangju Institute of Science and Technology (GIST), Gwangju, South Korea. His research interests include prognostics and health management and model verification and validation. Dr. Oh received the A. James Clark Fellowship (2007) and several awards including the IEEE PHM Data Challenge Competition Winner (2012), the PHM Society Data Challenge Competition Winner $(2014,2015)$, and the ACSMO Young Scientist Award (2016).

He received the B.S. degree in mechanical engineering from Korea University, Seoul, South Korea, in 2004, the M.S. degree in mechanical engineering from Korea Advanced Institute of Science and Technology, Daejeon, South Korea, in 2006, and the Ph.D. degree in mechanical engineering from the University of Maryland, College Park, MD, USA, in 2012. 


\section{Industry Forum:}

Moving from: "React and Repair" to "Predict and Prevent"

May $8-11,2018$

\section{Visualization Tools for PHM - Presentations \& Panel}

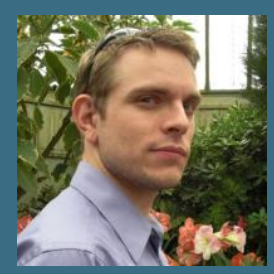

\section{Jeremy Marvel, Intelligent Systems Division, NIST}

Presentation Title: Visualization Tools for PHM: Metrics of Effective HMI

Bio: Jeremy A. Marvel is a research scientist and project leader at NIST. Dr. Marvel joined the Intelligent Systems Division at NIST in 2012, and has over thirteen years of robotics research experience in both industry and government. His research interests include intelligent and adaptive solutions for robot applications, with particular attention paid to human-robot and robot-robot collaborations, multirobot coordination, industrial robot safety, machine learning, perception, and automated parameter optimization. Dr. Marvel currently leads a team of scientists and engineers in metrology efforts at NIST toward collaborative robot performance, and developing tools to enable small and medium-sized enterprises to effectively deploy robot solutions.

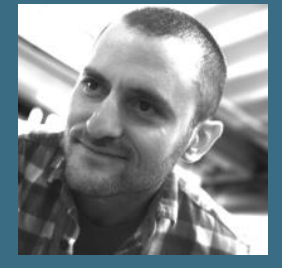

\section{Sinan Bank, Siemens Corporation}

Presentation Title: The Use of Digital Twin and Mixed Reality for Monitoring, Diagnostics, and Prognostics

Bio: Hasan Sinan Bank is a research scientist in Product Runtime Systems at SCCT, Princeton NJ. He has a track record of delivering high technology and intelligent solutions in Siemens and government projects including the project - Siemens Agile Manufacturing System (a.k.a. SpiderBots). He has more than 5 years of experience in software integration of mechatronics, control, optimization, and autonomous systems specifically with the focus of advanced manufacturing such as machining and laser-based additive manufacturing. He has earned recognition and contributed in the multi-criteria toolpath optimization of machining processes. He has $10+$ scientific publications and several patents in his domain of expertise including MxR implementations for manufacturing and control purpose.

\section{Demystifying Today's Al}

Michael Garris, Information Technology Laboratory, NIST

Bio: Michael Garris is a senior scientist and founding chair of the Artificial Intelligence (Al) Community of Interest at NIST where he has worked for the past 31 years with a technical focus in the areas of Al, image processing, pattern recognition, and biometrics. Mr. Garris serves on behalf of the Department of Commerce as co-chair for the President's National Science and Technology Council's (NSTC) Subcommittee on Machine Learning and Artificial Intelligence (ML/AI), and he served as member of the NSTC Networking and Information Technology Research and Development (NITRD) Subcommittee's Al Task Force. For 7 years in his career, Mr. Garris was privileged to manage the world-class biometric research, standards, test, and evaluation Image Group in NIST's Information Technology Laboratory (ITL). In 2003, Mr. Garris was part of a biometrics team which received the Department of Commerce Gold Medal Award. He has a BS in Computer Science from Clarion University of Pennsylvania, and a MS in Computer Science from Johns Hopkins. 


\section{Industry Forum:}

Moving from: "React and Repair" to "Predict and Prevent"

May $8-11,2018$

Industry Al -- A System Perspective in Machine Learning for Smart Manufacturing and Maintenance

Jay Lee, University of Cincinnati, Center for Intelligent Maintenance Systems

Bio: Professor Jay Lee is Ohio Eminent Scholar, L.W. Scott Alter Chair Professor, and Distinguished University Research Professor at the University of Cincinnati and is founding director of National Science Foundation (NSF) Industry/University Cooperative Research Center (I/UCRC) on Intelligent Maintenance Systems (www.imscenter.net) which is a multi-campus NSF Industry/University Cooperative Research Center which consists of the University of Cincinnati (lead institution), the University of Michigan, Missouri University of S\&T, and the University of TexasAustin. Since its inception in 2001, IMS Center has so far conducted more than 100 projects and has been supported by over 100 companies and research institutions worldwide including P\&G, GE Aviation, Boeing, Toyota, Nissan, Goodyear, Harley Davidson, Caterpillar, Siemens, Intel, Samsung, Bosch, National Instruments, Siemens, Chevron and many more. The cumulative benefit of IMS technologies was estimated to be \$1.4 Billion by 2015 .

His current research focuses on predictive big data analytics and cyber physical systems, prognostics and health management (PHM), and Industry 4.0 systems. He was selected to be one of the 30 Visionaries in Smart Manufacturing in U.S. by SME in Jan. 2016. In addition, he is co-Founder of Predictronics--a start-up company from NSF IMS Center of the Univ. of Cincinnati through NSF ICorp award in 2012 as well as a co-Founder of CyberInsight Technology in 2016.

\section{Planning for the Future - Building and Leveraging Artificial Intelligence -} Panel Discussion

Michael Garris, Information Technology Laboratory, NIST

Jay Lee, University of Cincinnati, Center for Intelligent Maintenance Systems 


\section{Appendix C - Participant List}

\begin{tabular}{|c|c|}
\hline NAME & ORGANIZATION \\
\hline Al Salour & The Boeing Company \\
\hline Al Wavering & NIST \\
\hline Alex Klinger & NIST \\
\hline Alexander Buddenbaum & Foxconn Technology Group \\
\hline Allison Barnard Feeney & NIST \\
\hline Anand Kandaswamy & NIST \\
\hline Andrew Hess & The Hess PHM Group \\
\hline Andrew Stettner & The Century Foundation \\
\hline Bill Bernstein & NIST \\
\hline Brian A. Weiss & NIST \\
\hline Brittany Newell & Purdue University \\
\hline Byeng Dong Youn & Seoul National University \\
\hline Byungchang Jung & Korea Institute of Machinery and Materials \\
\hline Chabum Lee & Tennessee Technological University \\
\hline Chan Hee Park & Seoul National University \\
\hline Chao Yang Kao & Foxconn \\
\hline Cheryl Xu & Florida State University \\
\hline Craig Schlenoff & NIST \\
\hline Cynthia Kirby & ATI \\
\hline David Butry & NIST \\
\hline David Sieel & Predictronics \\
\hline Dean Bartles & University of New Hampshire \\
\hline Dick Tiano & ATI \\
\hline Donnie Alonzo & ASME \\
\hline Doug Thomas & NIST \\
\hline Douglas Hart & Emerson \\
\hline Ed Spence & The Machine Instrumentation Group \\
\hline Eric Belski & Aerotech, Inc. \\
\hline Euiyoung Kim & Korea Institute of Machinery \&amp; Materials \\
\hline Fu-Chiang Hsu & Foxconn \\
\hline Gerald Reid & Foxconn \\
\hline Gregory Vogl & NIST \\
\hline Helen Qiao & NIST \\
\hline Howard Harary & NIST \\
\hline Hyunbo Cho & POSTECH \\
\hline Hyunseok Oh & Gwangju Institute of Science and Technology \\
\hline
\end{tabular}




\begin{tabular}{|c|c|}
\hline NAME & ORGANIZATION \\
\hline Jaime Camelio & CCAM \\
\hline James Daggon & Rice Lake Weighing Systems \\
\hline James Moyne & Applied Materials \\
\hline Jay Lee & Univ. of Cincinnati \\
\hline Joel Neidig & ITAMCO \\
\hline Jorge Arinez & General Motors \\
\hline Jose Colucci & NIST MEP \\
\hline Jose Hernandez & GSS \\
\hline Jules McGuire & AMT - Association for Manufacturing Technology \\
\hline Junmin Lee & Seoul National University \\
\hline Justinian Rosca & Siemens Corporation, Corporate Technology \\
\hline Kai Goebel & NASA ARC \\
\hline Karl Rajotte & Tecon, Inc \\
\hline Karl Reichard & Applied Research Lab \\
\hline KC Morris & NIST \\
\hline Kevin Jurrens & NIST \\
\hline Kim Small & Reliability Insights \\
\hline Kirk Dohne & NIST \\
\hline Kyung Ho Sun & Korea Institute of Machinery \&amp; Materials \\
\hline Logen Johnson & SAE International \\
\hline Lowell Jones & CMXG/MXDEC \\
\hline Madhusudanan Navinchandran & NIST \\
\hline Mark Walker & D2K Technologies \\
\hline Michael Armacost & Applied Materials \\
\hline Michael Brundage & NIST \\
\hline Michael Molnar & NIST \\
\hline Michael Plesnarski & R-V Industries, INC \\
\hline Michael Sharp & NIST \\
\hline Miguel Saez-Graterol & University of Michigan \\
\hline Moneer Helu & NIST \\
\hline Nancy Diaz-Elsayed & University of South Florida \\
\hline Nicholas Duncan & Aerotech Inc \\
\hline Palamadai Seshan & 5G Technologies Ltd. \\
\hline Paul Chen & Foxconn Interconnect Technology \\
\hline Prabhu Jackson & Cummins Inc \\
\hline Puwei Huang & Foxconn \\
\hline Qing Chang & Stony Brook University \\
\hline
\end{tabular}




\begin{tabular}{|l|l|}
\hline NAME & ORGANIZATION \\
\hline Radu Pavel & TechSolve, Inc. \\
\hline Ravi Rajamani & drR2 consulting \\
\hline Richard Arnold & CyberPoint International, LLC \\
\hline Robert Andes, Jr. & TDKC \\
\hline Robert Gao & Case Western Reserve University \\
\hline Sanket Amberkar & Falkonry \\
\hline Sascha Moccozet & NIST \\
\hline Satoshi Nagahara & NIST \\
\hline Scott Sipe & MANTEC \\
\hline Scott Truitt & ATI \\
\hline Simon Frechette & NIST \\
\hline Sinan Bank & Siemens Corporation Corporate Technology \\
\hline Stanley Chu & Foxconn \\
\hline Stephen Ludwick & Aerotech, Inc. \\
\hline Steven Weinman & ASME \\
\hline Thomas Fiske & Yokogawa \\
\hline Thorsten Wuest & West Virginia University \\
\hline Thurston Sexton & NIST \\
\hline Tim Sprock & NIST \\
\hline Todd Sabin & Maryland Department of Commerce \\
\hline Tom Hedberg & NIST \\
\hline Tom Zbell & Genedge \\
\hline Tommi Makila & Energetics \\
\hline Tsubasa Watanabe & Hitachi America, Ltd. \\
\hline Vijay Srinivasan & NIST \\
\hline William Sobel & VIMANA \\
\hline William Walker & D2K Technologies \\
\hline Yannick Tamm & Energetics \\
\hline Ying Hsin Andrew Liou & Foxconn Precision \\
\hline Zhijun Liu & Makino Inc \\
\hline & \\
\hline
\end{tabular}




\section{Appendix D - NIST Presentations}

The following slide decks are included in this section in their entirety and in order of their presentation in this report:

- Kirk Dohne - "Overview of the NIST Engineering Laboratory” (Section 2.1)

- Michael Molnar - "Manufacturing USA and DMDII - Program Update and Activities in PHM" (Section 3.1)

- Al Wavering - "NIST Smart Manufacturing Programs: Driving Innovation and Reducing Risks of Adoption of New Technologies" (Section 3.2)

- Doug Thomas - "The Costs and Benefits of Advanced Maintenance in Manufacturing" (Section 4.1)

- Greg Vogl - "Emerging Sensing Technologies towards Smart Machine Tools" (Section 6.2.4)

- Moneer Helu - "Connecting and Deploying Smart Manufacturing Technology to Support PHM” (Section 6.4.2)

- Michael Brundage - Using Unstructured Work Order Data to Improve Maintenance Procedures in Manufacturing" (Section 7.1)

- Brian A. Weiss - "NIST Research on Monitoring, Diagnostics, and Prognostics for Manufacturing Workcells" (Section 7.2)

- Tom Hedberg - "Challenges in Standards for a Model-Based Enterprise” (Section 8.2.4)

- Jeremy Marvel - "Visualization Tools for PHM: Metrics of Effective HMI" (Section 10.1)

- Michael Garris - "Demystifying Today’s Artificial Intelligence” (Section 11.1) 


\section{Overview of the \\ NIST Engineering Laboratory}

Kirk Dohne, Associate Director

Engineering Laboratory 


\section{NIST Mission}

To promote U.S. innovation and industrial competitiveness by advancing measurement science, standards, and technology in ways that enhance economic security and improve our quality of life

measurement science

Creating the experimental and theoretical tools - methods, metrics, instruments, and data - that enable innovation

\section{standards}

Disseminating physical standards and providing technical expertise to documentary standards that enable comparison, ensure interoperability, and support commerce technology

Driving innovation through knowledge dissemination and public-private partnerships that bridge the gap between discovery and the marketplace 


\section{EL Mission}

To promote U.S. innovation and industrial competitiveness

by advancing measurement science, standards, and technology for engineered systems in ways that enhance economic security and improve our quality of life

measurement science

Creating the experimental and theoretical tools - methods, metrics, instruments, and data - that enable innovation

standards

Disseminating physical standards and providing technical expertise to documentary standards that enable comparison, ensure interoperability, and support commerce technology

Driving innovation through knowledge dissemination and public-private partnerships that bridge the gap between discovery and the marketplace 


\section{NIST at a Glance}
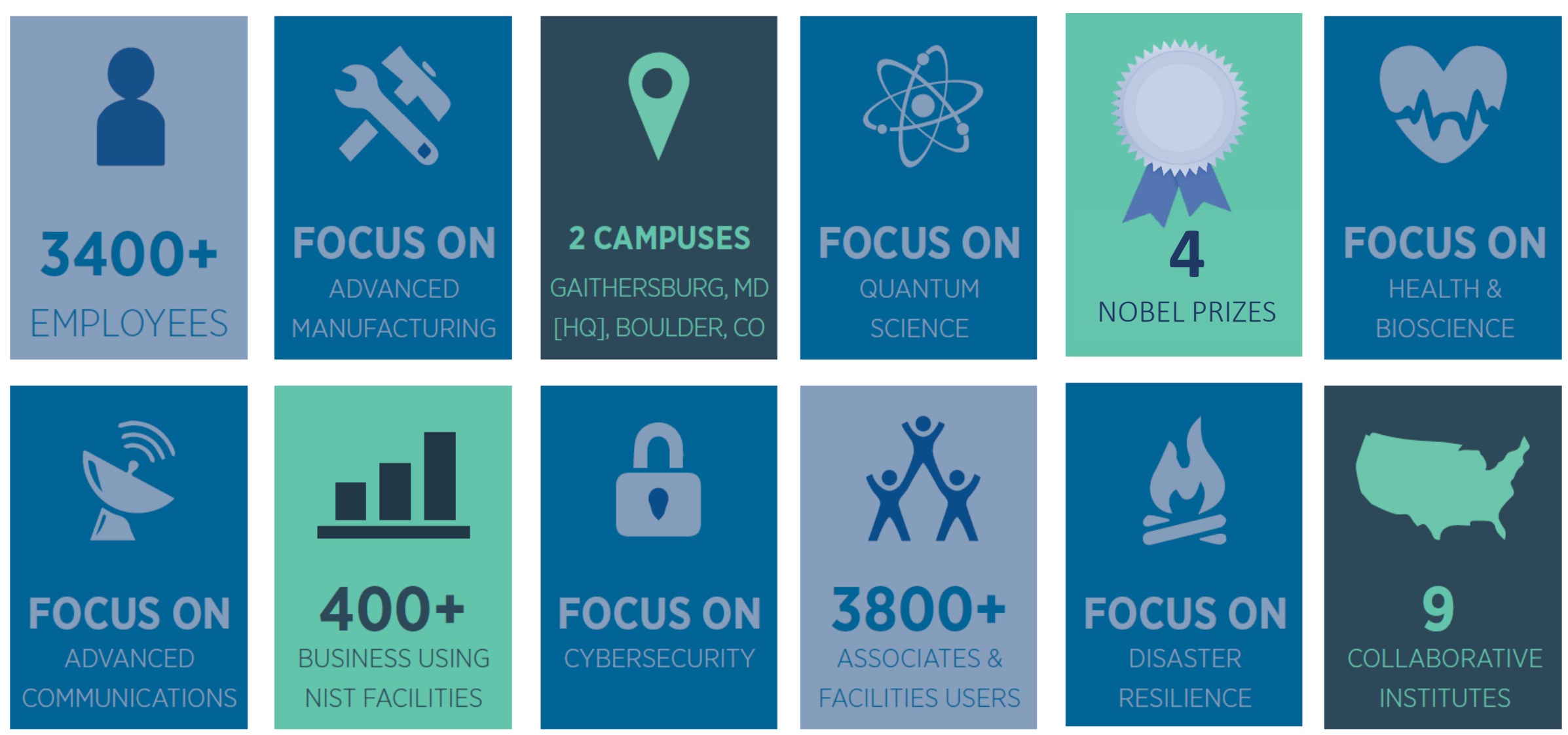

www.nist.gov 


\section{NIST's reputation: our biggest strength}

NIST's reputation allows it to punch above its weight

- NIST is recognized as having deep technical excellence

- NIST is seen as an uncompromising measurement science laboratory, the best in the world

- NIST is known for its neutrality, providing unbiased results

- NIST is industry-focused, providing extensive ties to companies, consortia and associations

- NIST is non-regulatory and doesn't make (but can inform) policy, allowing open discussions with stakeholders

NIST's reputation for excellence and neutrality provides a means for convening diverse stakeholders to address complex technical issues

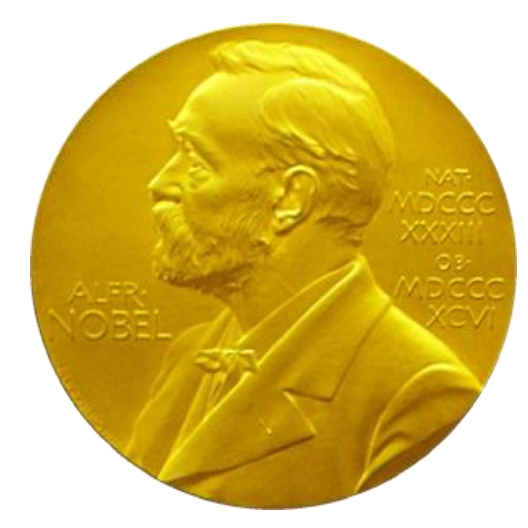




\section{Measurements are Critical to Commerce}

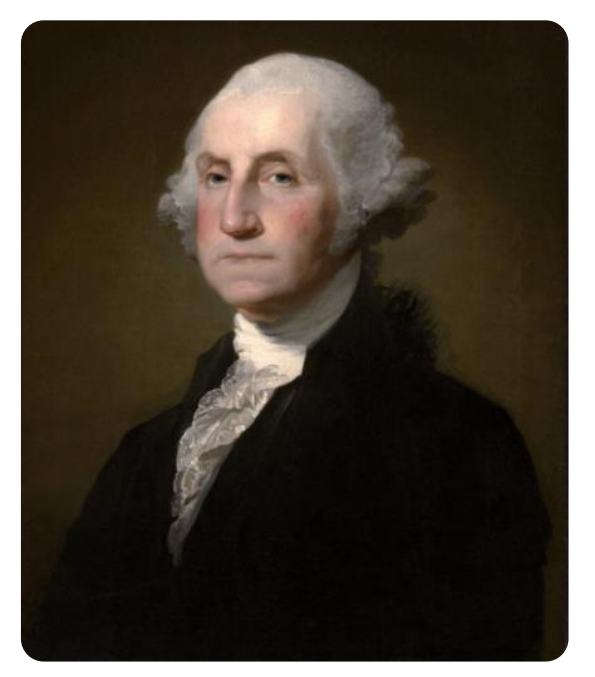

"Uniformity in the currency, weights, and measures of the United States is an object of great importance, and will, I am persuaded, be duly attended to."

George Washington, State of the Union Address, 1790

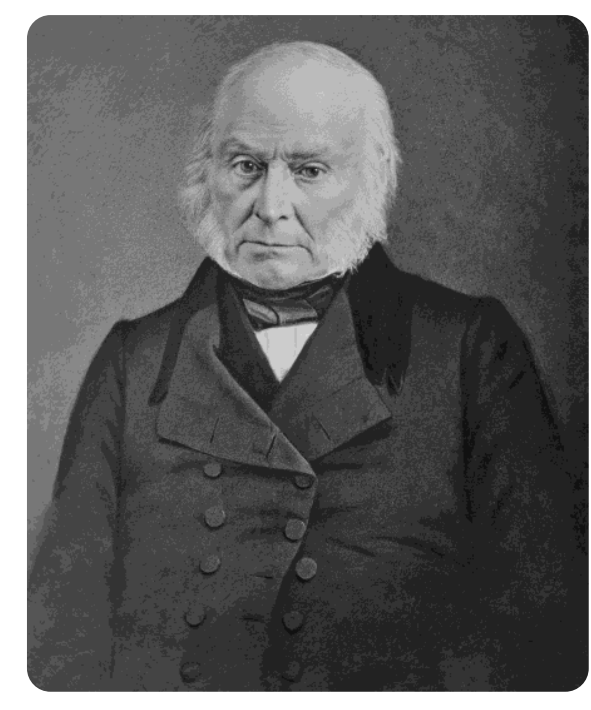

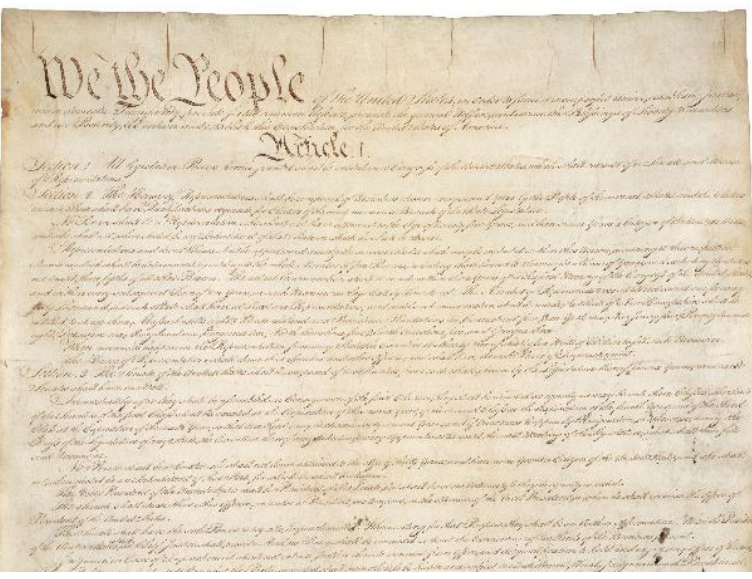

"The Congress shall have the power to...fix the standard of weights and measures"

Article I Section 8, 1789
"Weights and measures may be ranked among the necessities of life to every individual of human society." John Quincy Adams, 1821 


\section{Measurements are Critical to Innovation}

- If you know how to measure something, you can design it, improve it, and compare it

- NIST measurement science provides foundation for innovation in every industry and economic sector, from manufacturing to health care to defense

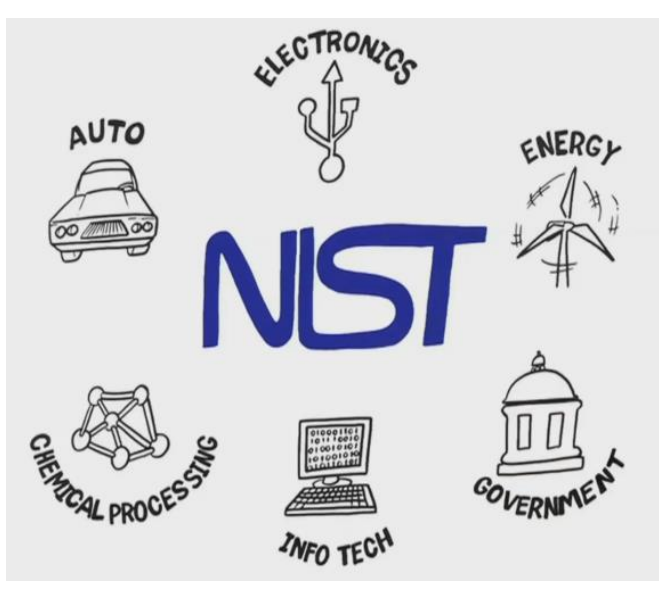




\section{NIST's Unique Role in Documentary Standards}

Providing support to industry and government for voluntary standards development

\section{NIST's unique role}

- NIST coordinates standards policy among federal agencies (National Technology Transfer and Advancement Act, 1996)

- NIST Director is President's principal advisor on standards (American Innovation and Competitiveness Act, 2016)

- NIST's laboratory expertise provides measurement-based and unbiased data to improve decision-making in standards bodies

\section{Expert participation}

- $400+$ NIST technical staff in 100+ standard committees

- Leadership in international standards bodies such as ASTM, IEEE, ISO, IEC

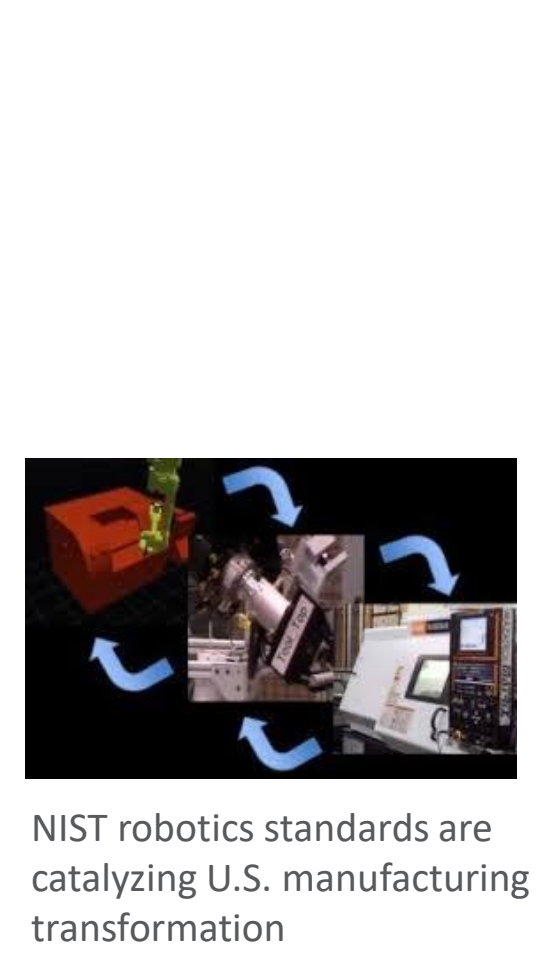

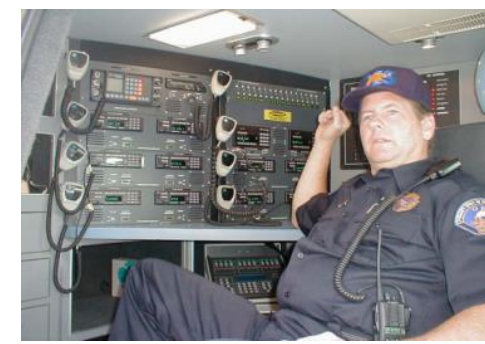

Standards and conformity assessment requirements

for public safety comms equipment is transforming emergency response

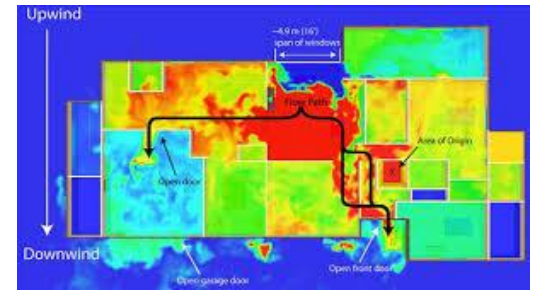

NIST studies of fire behavior led to changes in U.S. building codes, which saved lives 


\section{NIST Laboratory Programs}

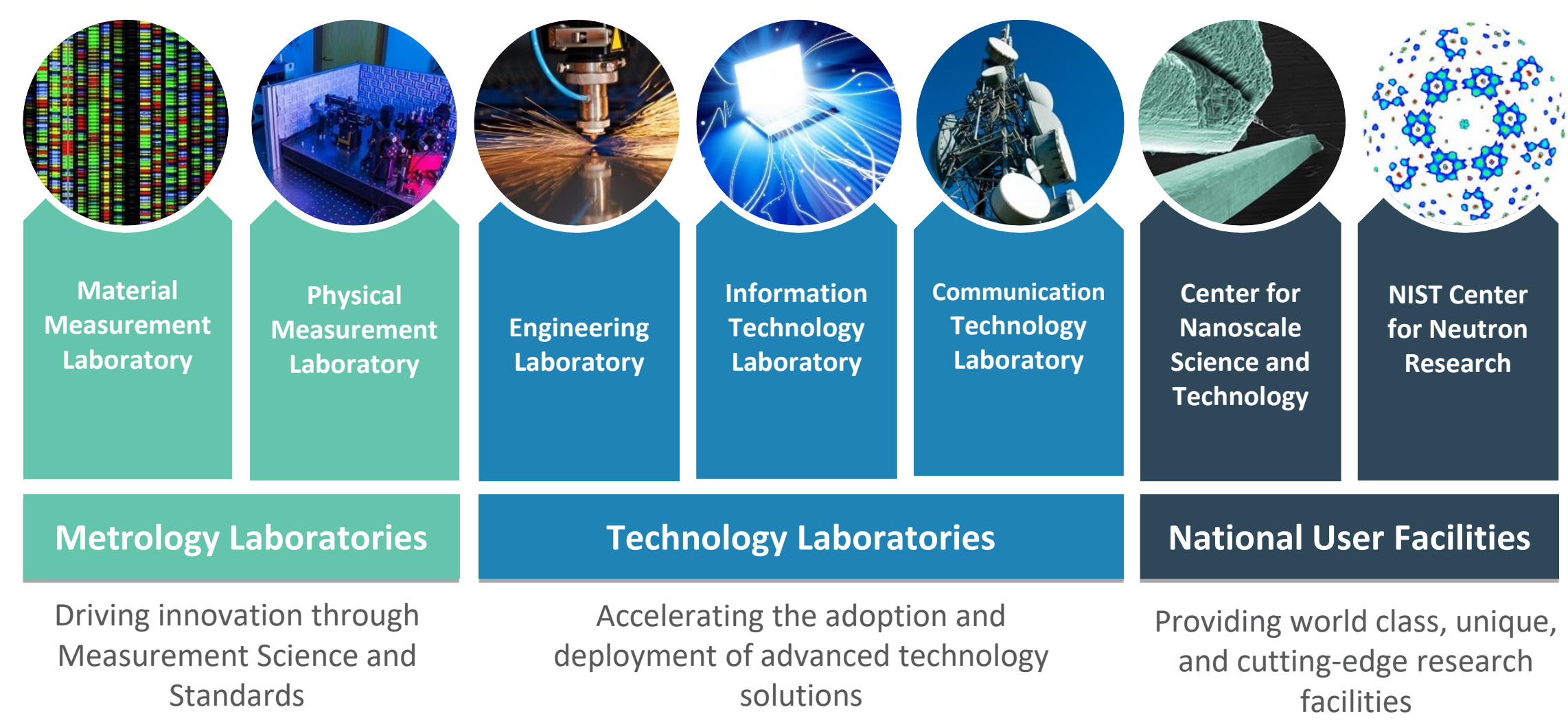




\section{NIST Laboratory Programs}

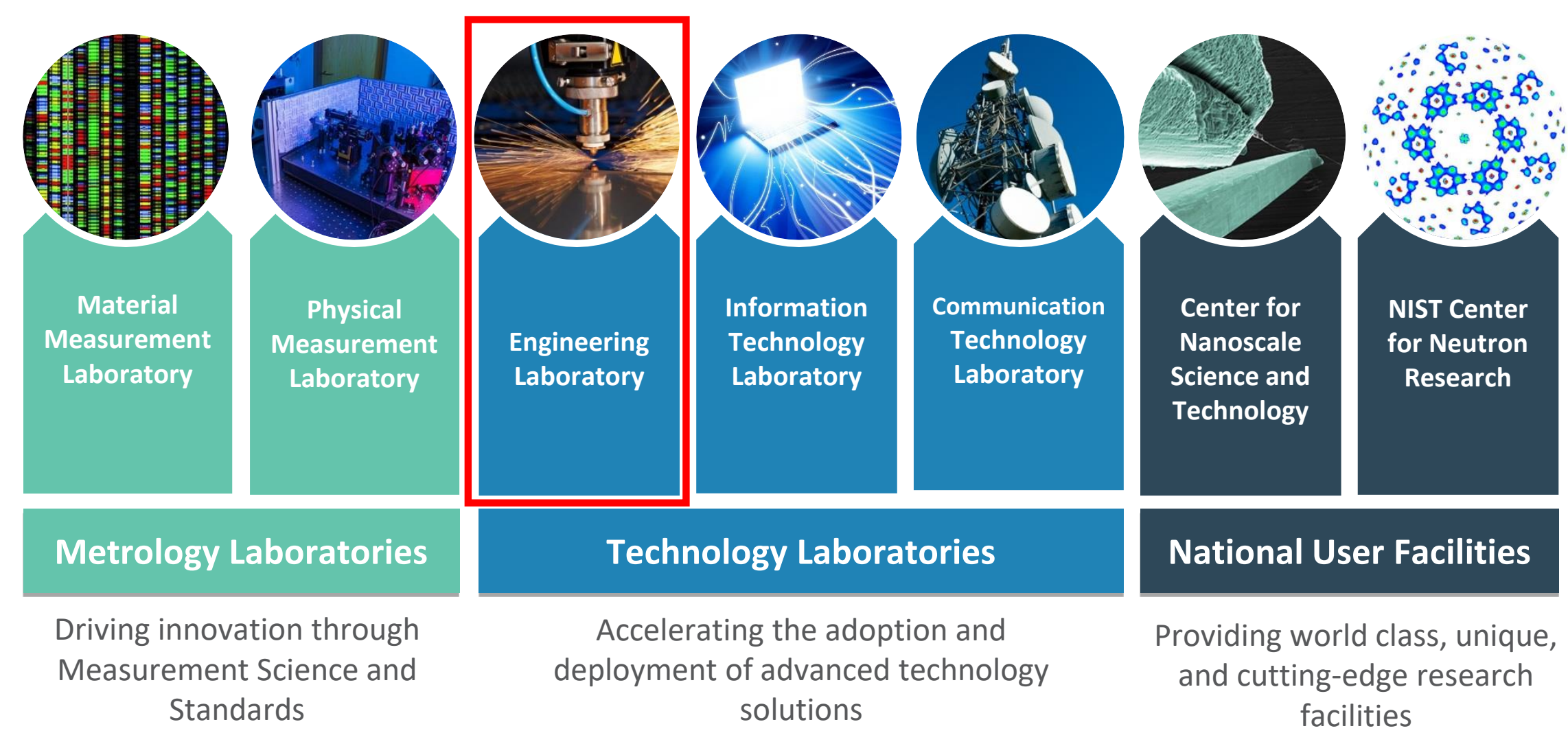




\section{Focus on Smart Manufacturing}

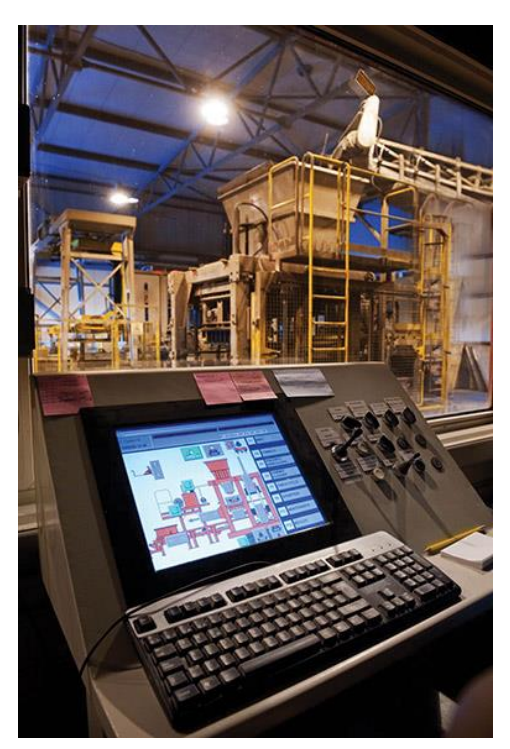

Smart Manufacturing Operations

Planning and Control

Smart Manufacturing Systems Design and Analysis
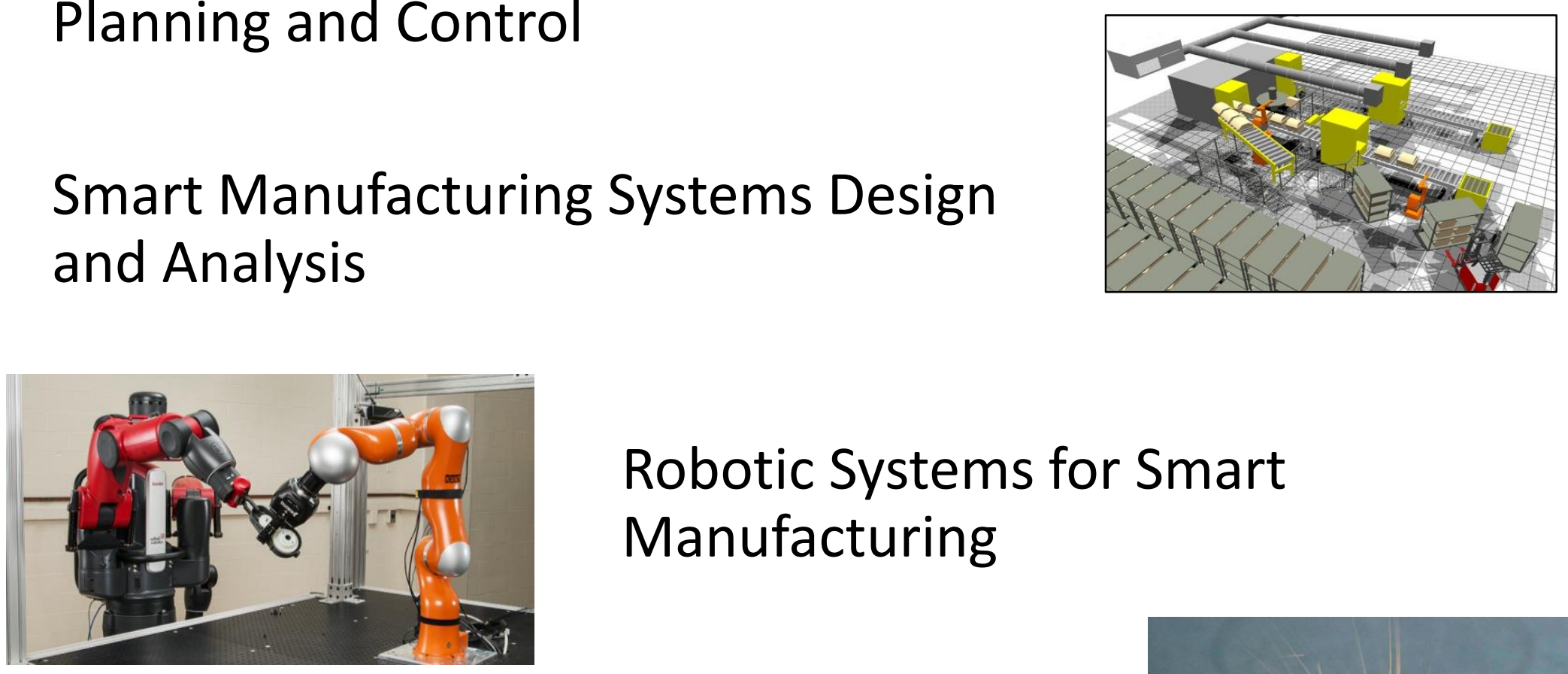

Robotic Systems for Smart Manufacturing

Measurement Science for Additive Manufacturing

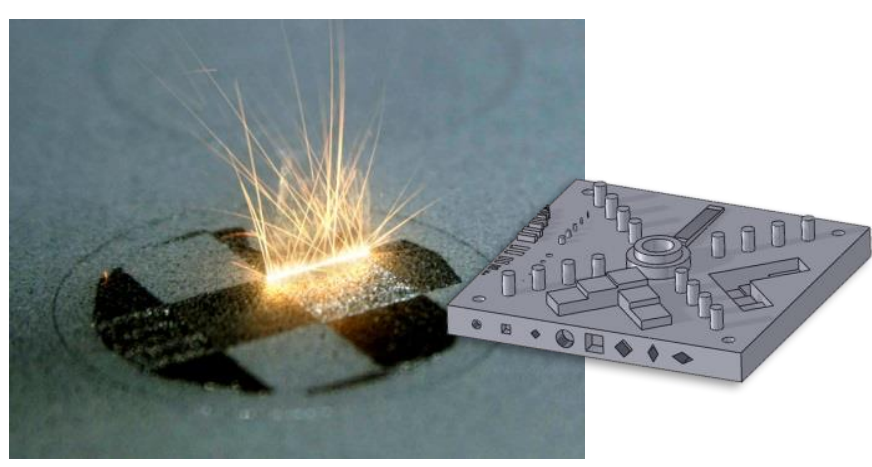




\section{NIST and Advanced Manufacturing}

A partner to US manufacturers sector for more than a century, NIST helps the nation's manufacturers to invent, innovate, and create by:

- Precision measurements - manufacturers use NIST test methods, measurement tools, and scientific data every day

- Advanced materials - NIST is building a materials infrastructure to accelerate the timeline from design to deployment of new materials

- Partnerships - collaborations with the private sector and academic organizations help advance and disseminate research and support US manufacturers

"It is therefore the unanimous opinion of your committee that no more essential aid could be given to manufacturing [...] than by the establishment of the [National Bureau of Standards]."

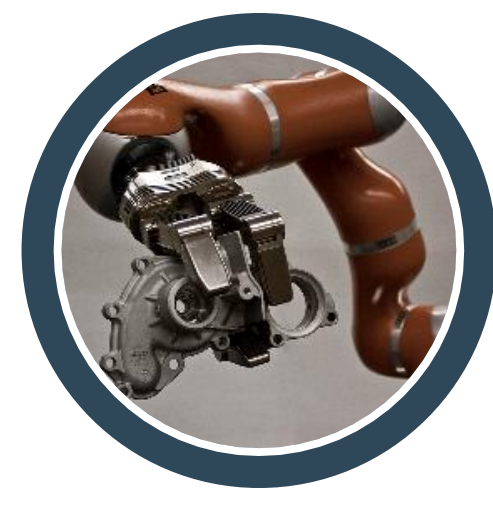

Manufacturing USA is a nationwide network of public-

private institutes to meet technical needs and create tomorrow's workforce

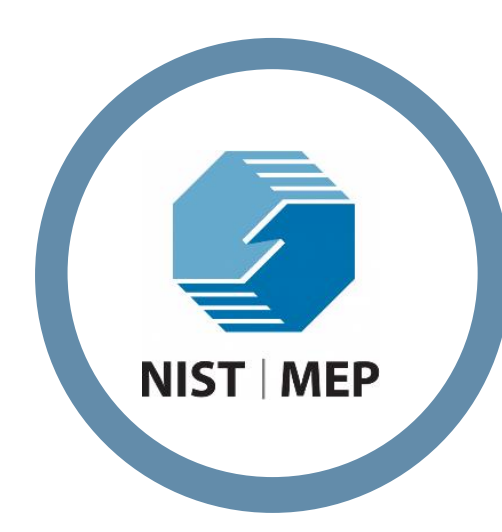

NIST labs develop

measurements and tools for areas including robotics performance

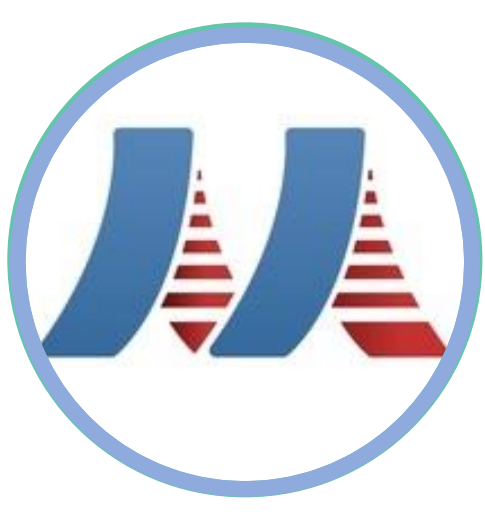

Hollings Manufacturing Extension Partnership centers in every US state provide services to small and medium manufacturers 


\section{Final Message}

- NIST and the Engineering Laboratory play an essential Federal government role by developing new measurement science, standards, and technology throughout manufacturing.

- Our role is to underpin standards and trade that supports US companies and helps them get better products to market faster.

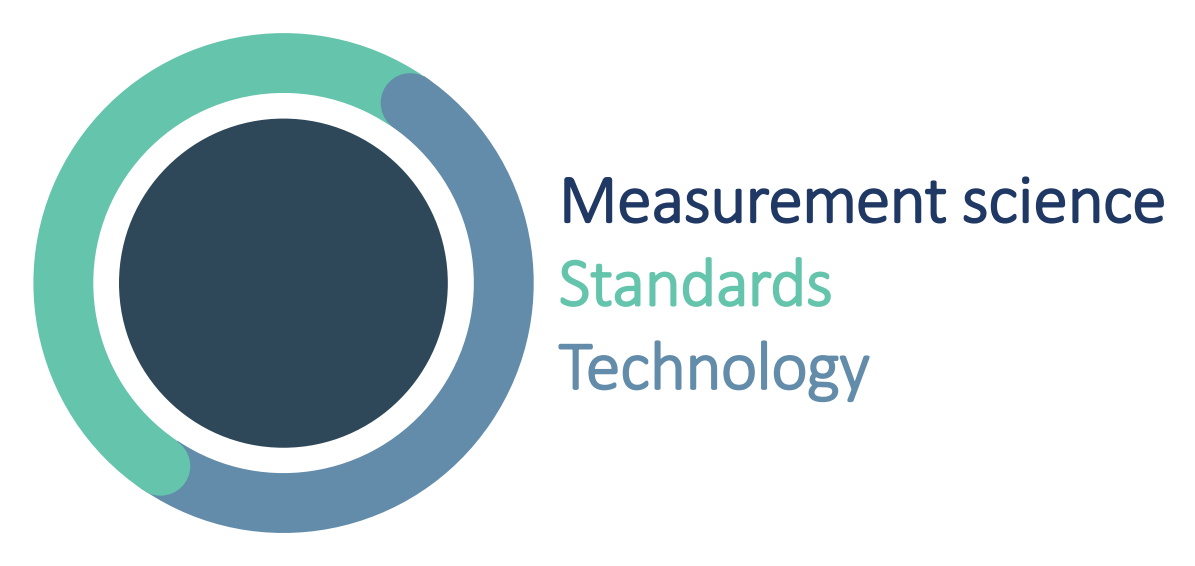




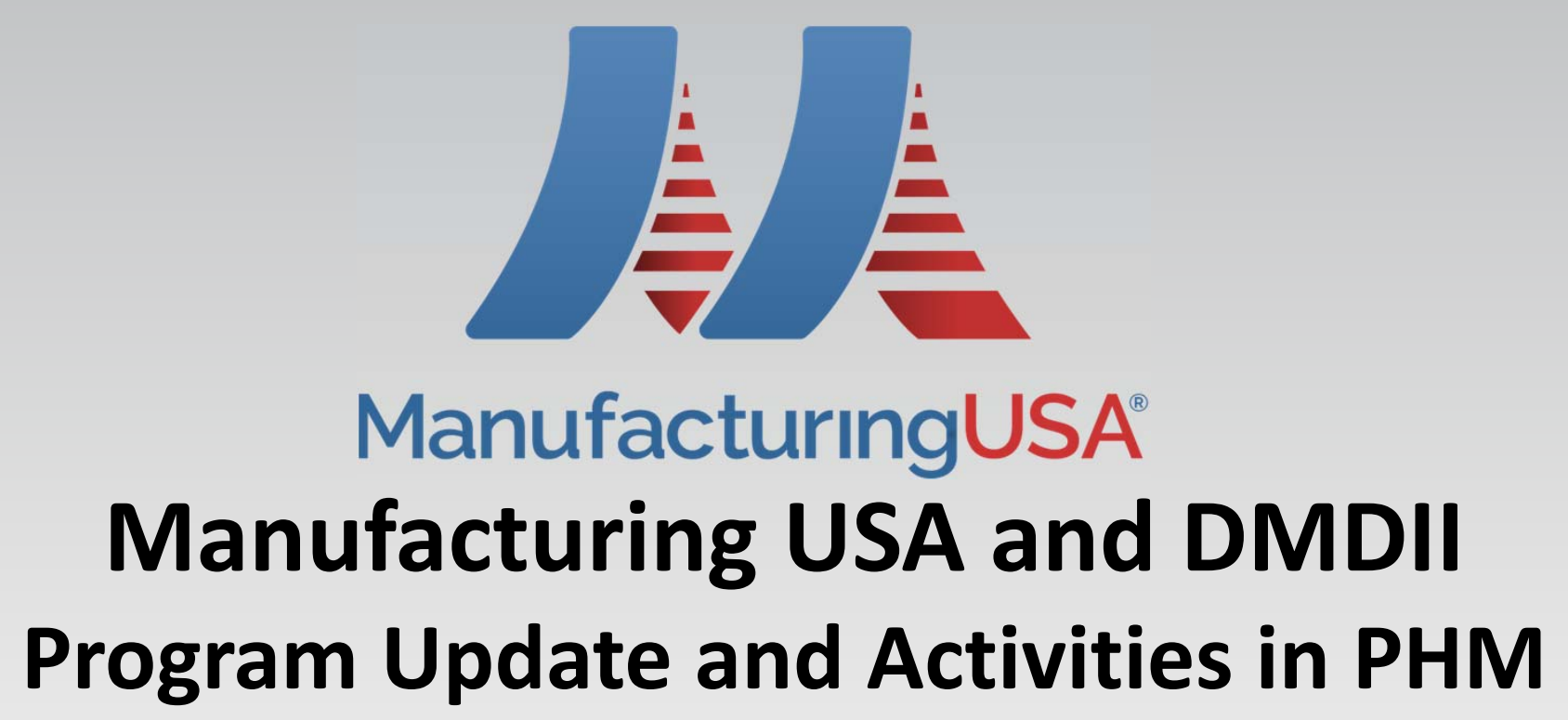

NIST Industry Forum on Machine Monitoring, Diagnostics and Prognostics May 8, 2018

Mike Molnar

Advanced Manufacturing National Program Office

An interagency team building partnerships with U.S. Industry and Academia

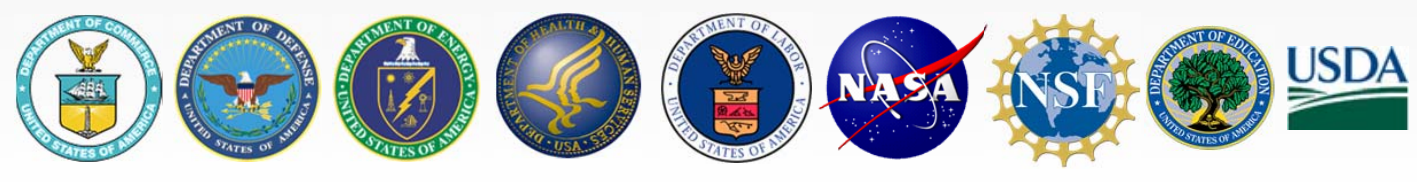




\section{Agenda}

- Manufacturing USA ${ }^{\circledR}$ Overview

- How an Institute Works: DMDII

- Delivering Value: 2017 Results Highlights

- Example PHM Projects 


\section{Why Manufacturing USA}

U.S. Trade Balance for Advanced Technology Products

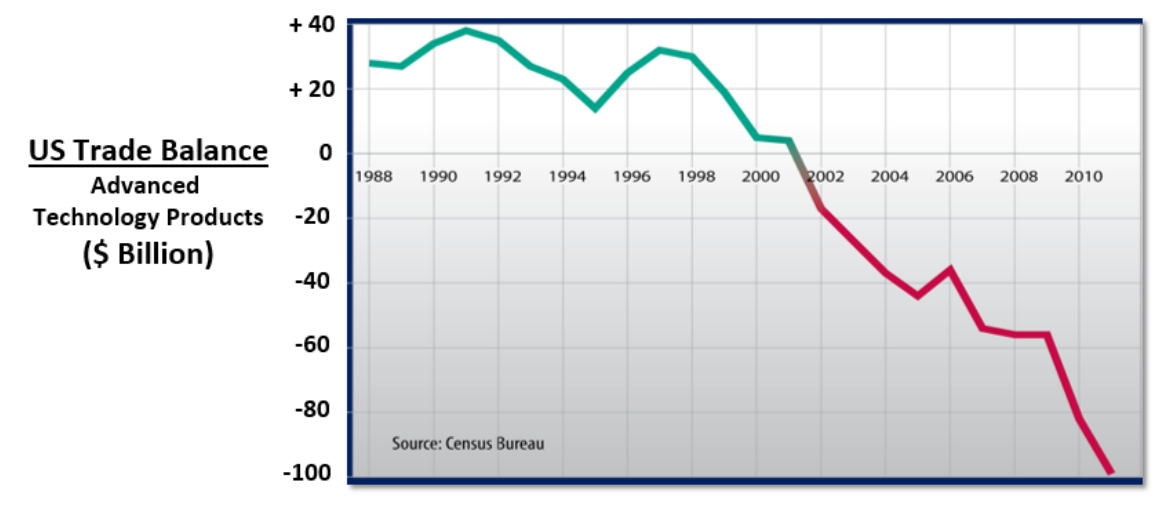

President's Council of Advisors on Science and Technology

- Advanced Manufacturing Partnership: 2011-2012

- Advanced Manufacturing Partnership 2.0: 2013-2014

\section{Revitalize American Manufacturing and Innovation Act}

- 118 bipartisan co-sponsors!

- signed into law December 16, 2014

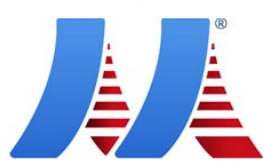
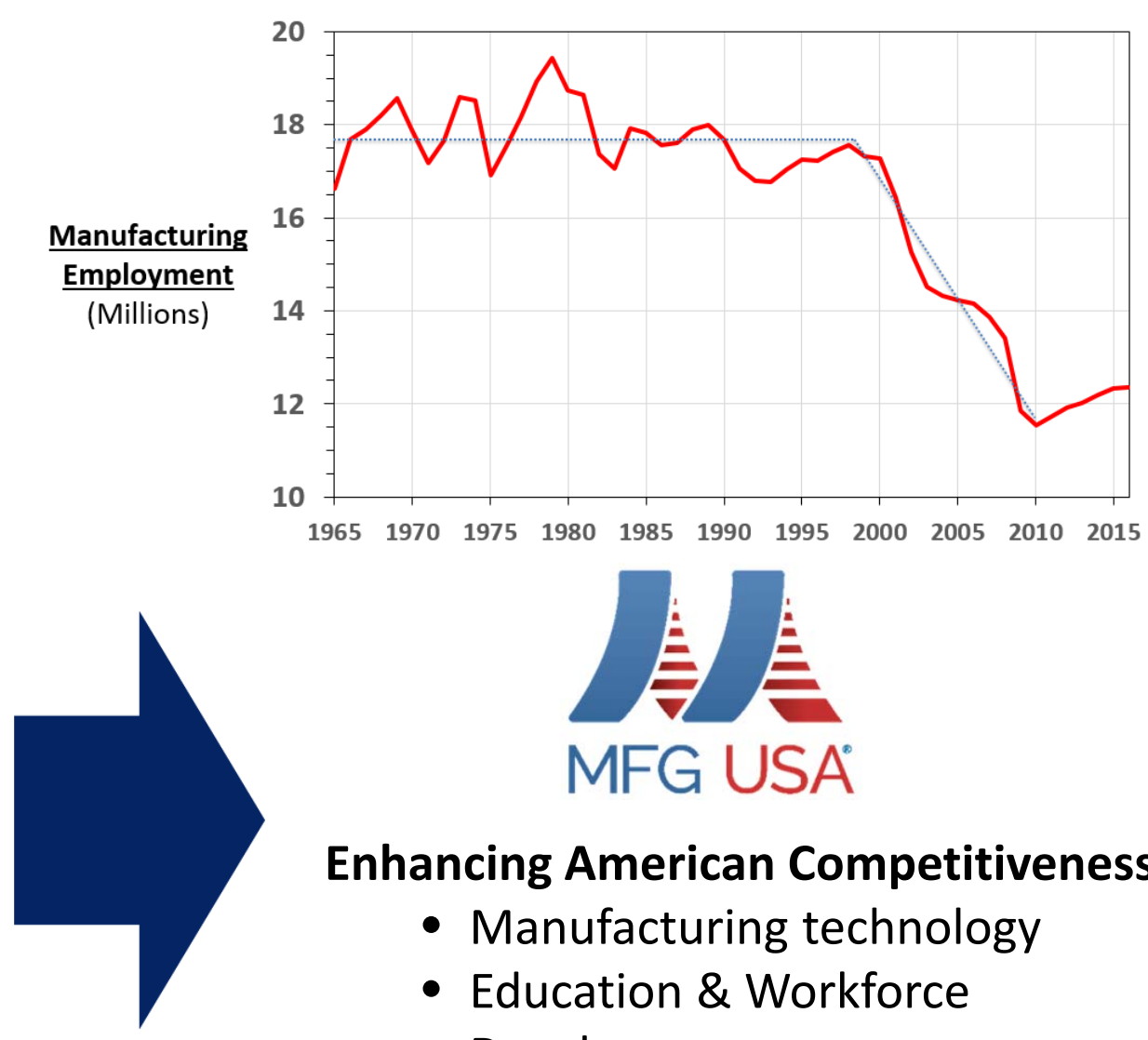

Enhancing American Competitiveness by

- Manufacturing technology

- Education \& Workforce Development 


\section{Manufacturing USA: A Public-Private Partnership}

President's Council of Advisors on Science and Technology

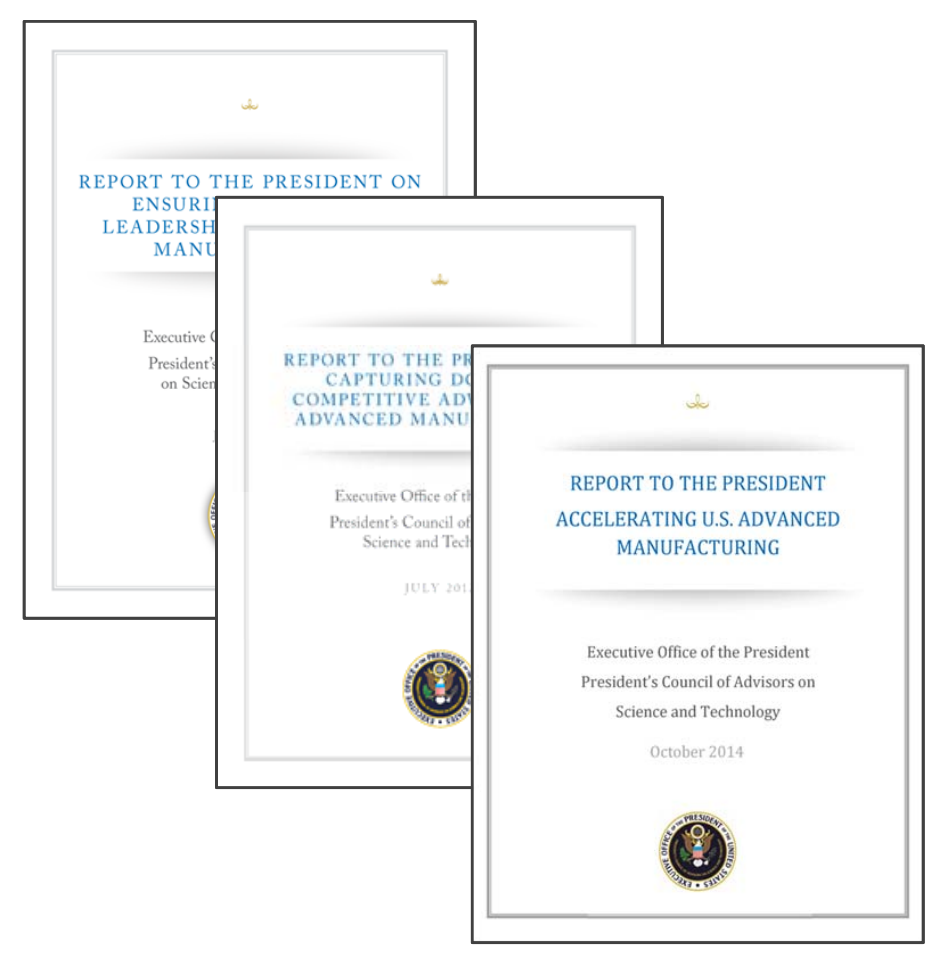

Market Failure in

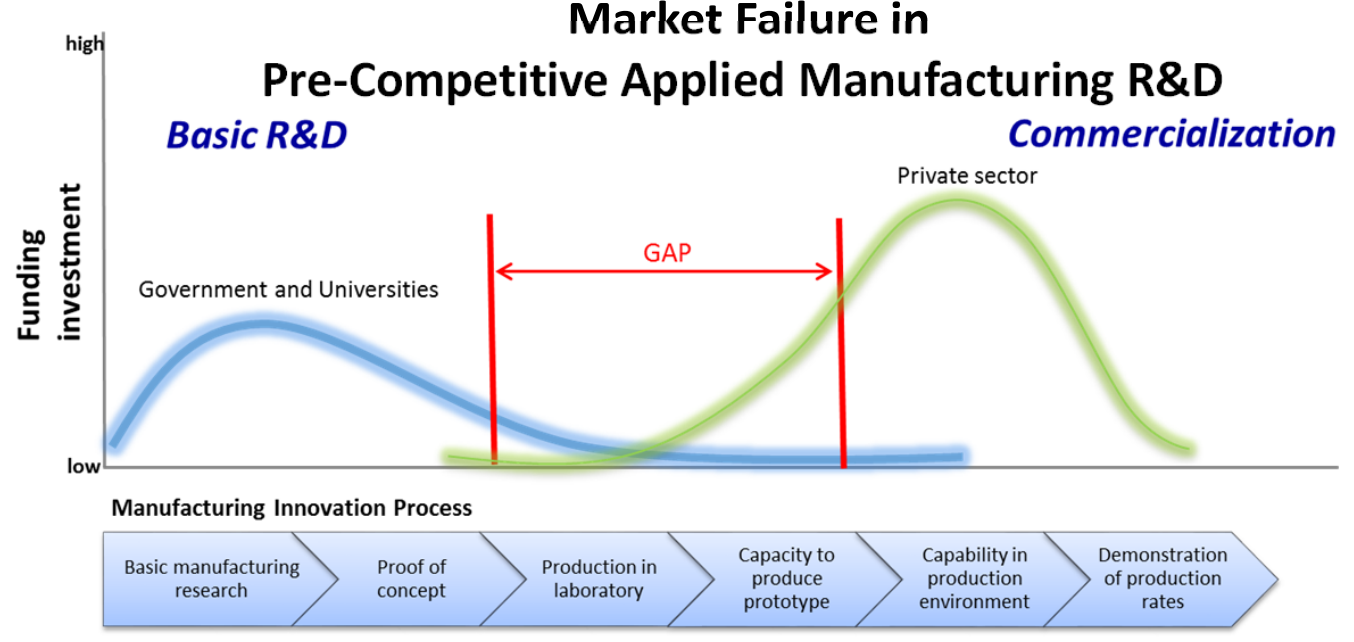

VISION

U.S. global leadership in advanced manufacturing

\section{MISSION}

Connecting people, ideas, and technology to solve industryrelevant advanced manufacturing challenges, thereby enhancing industrial competitiveness and economic growth and strengthening our national security.

\begin{tabular}{|c|c|c|}
\hline PROGRAM GOALS \\
\hline \multicolumn{3}{|c|}{ Competitiveness } \\
\hline $\begin{array}{c}\text { Technology } \\
\text { Advancement }\end{array}$ & $\begin{array}{c}\text { Workforce } \\
\text { Development }\end{array}$ & $\begin{array}{c}\text { Technology } \\
\text { Sustainability }\end{array}$ \\
\hline
\end{tabular}




\section{The Institute Design}

\section{Creating the space for Industry \& Academia to collaborate}

Institute Framework

Design published

January 2013

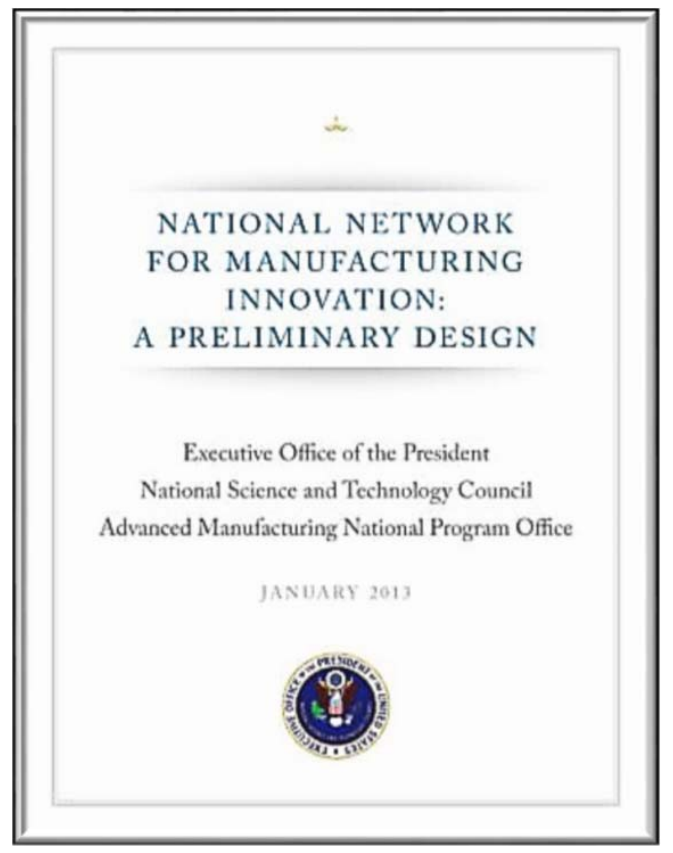

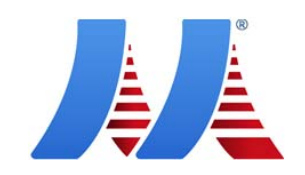

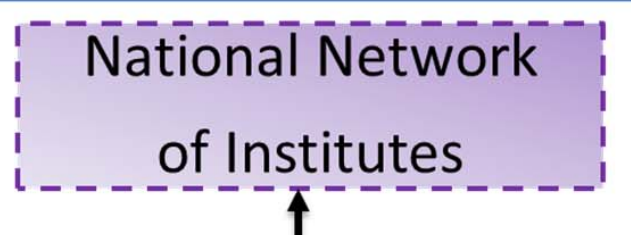

Academia

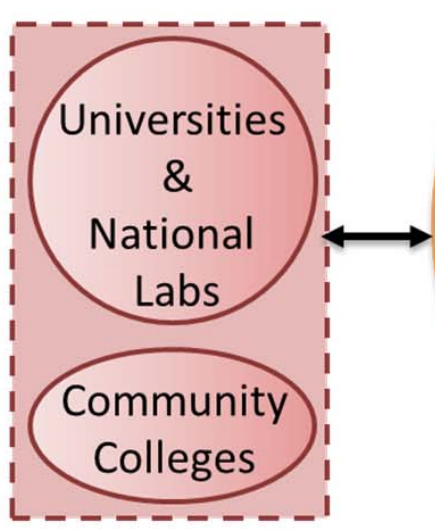

Institute

For Manufacturing Innovation

Prototype lab/shops

Research facility

Computer lab

Shared Use

Facility

$\downarrow$

Government

Industry

Large

Manufacturing

Companies

Small \&

Medium

Enterprise

Start-ups

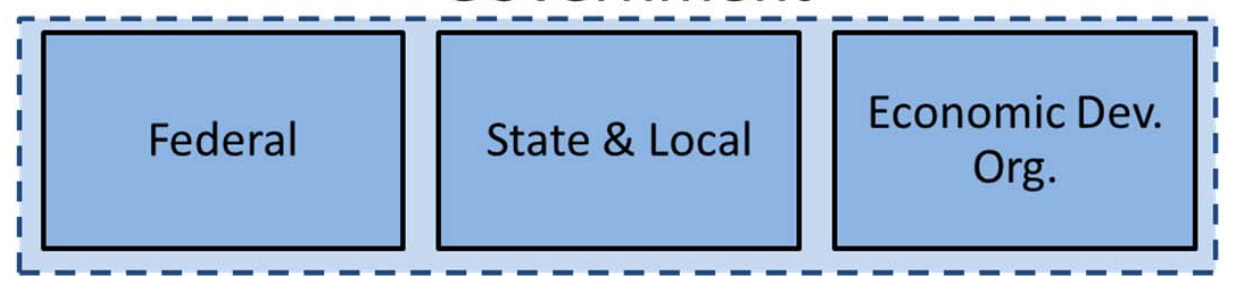




\section{Manufacturing USA Today}

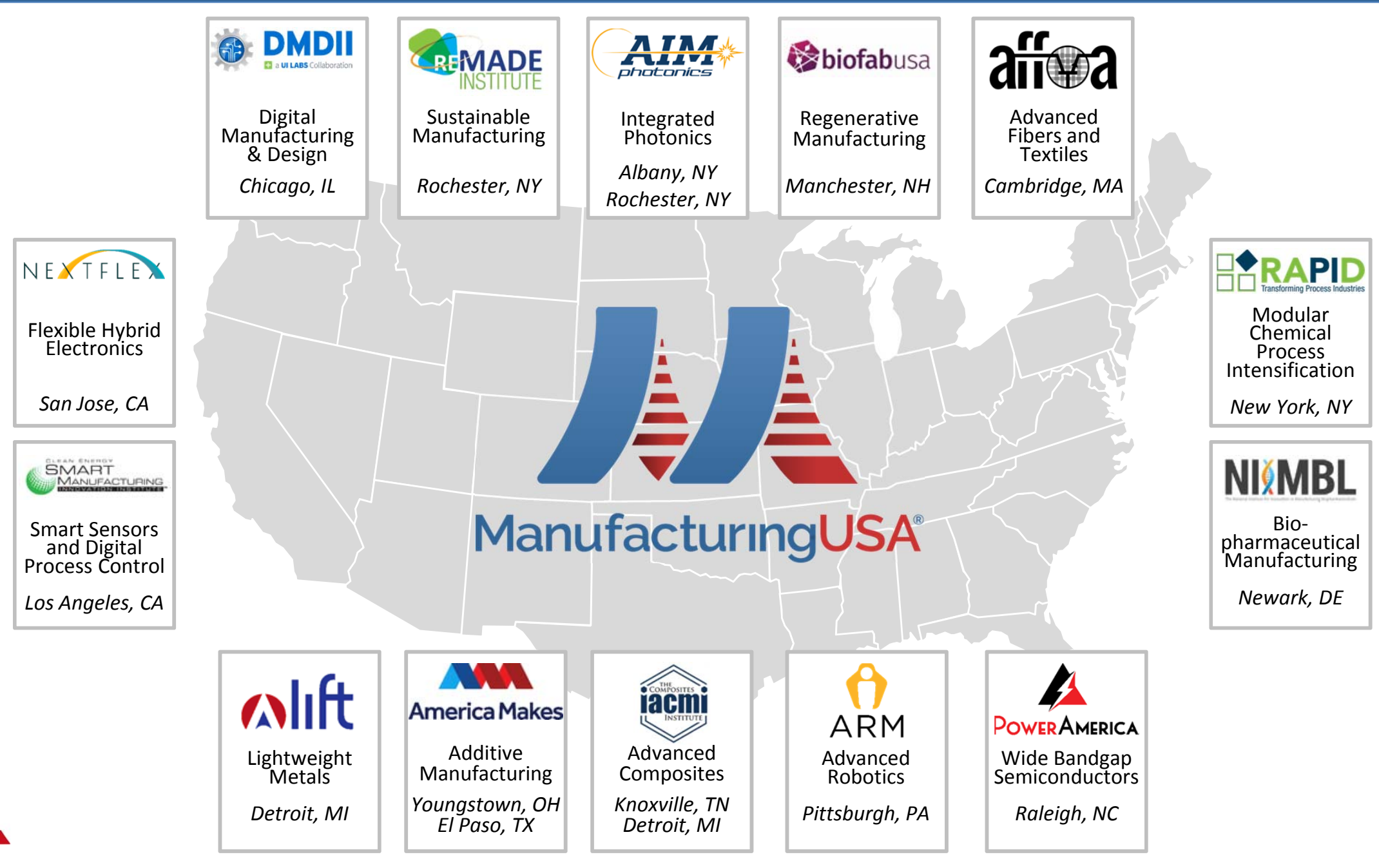




\section{Agenda}

- Manufacturing USA Overview

- How an Institute Works - DMDII

- Delivering Value: 2017 Results Highlights

- Example PHM Projects 


\section{1) Each Institute has a clear mission based on a critical}

Industry need

\section{DMDII exists to transform American} manufacturing competitiveness by accelerating the development and adoption of digital technology across the manufacturing enterprise 


\section{2) Each Institute creates value for industry participation and funding}

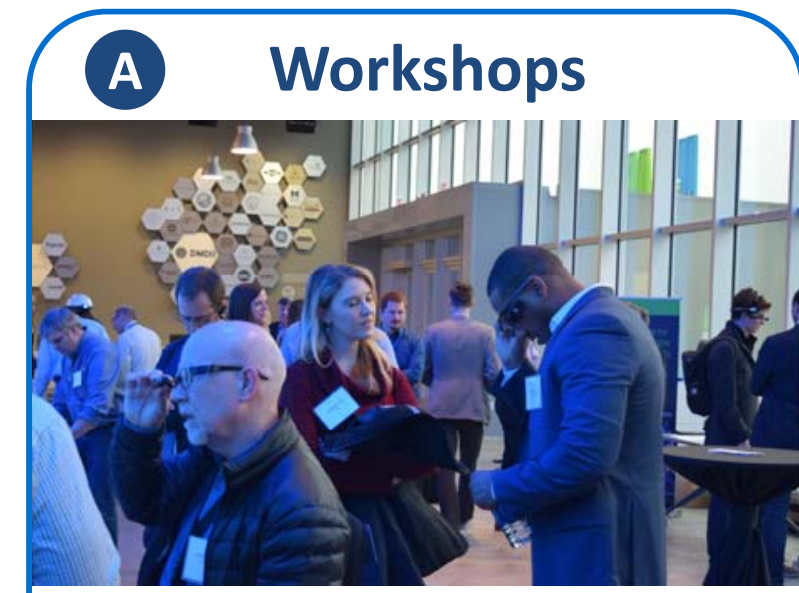

Topic-focused sessions where partners engage in solution oriented discussions to drive projects and investments

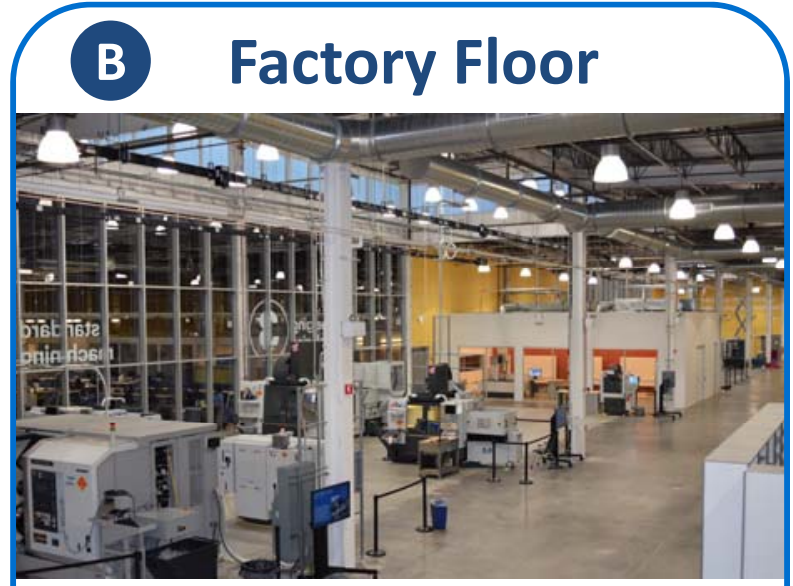

Creating an experiential manufacturing environment to demo, test \& prove a wide variety of DM\&D technologies

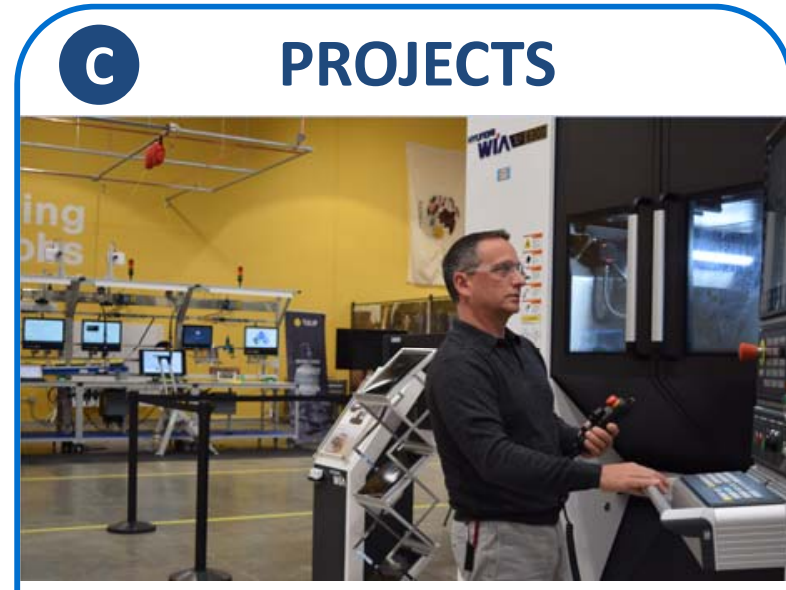

Applying the DMDII workshop and technical outcomes into real world applications

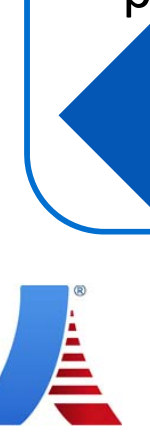




\section{3) Each Institute creates an effective collaboration space for pre-competitive applied R\&D}
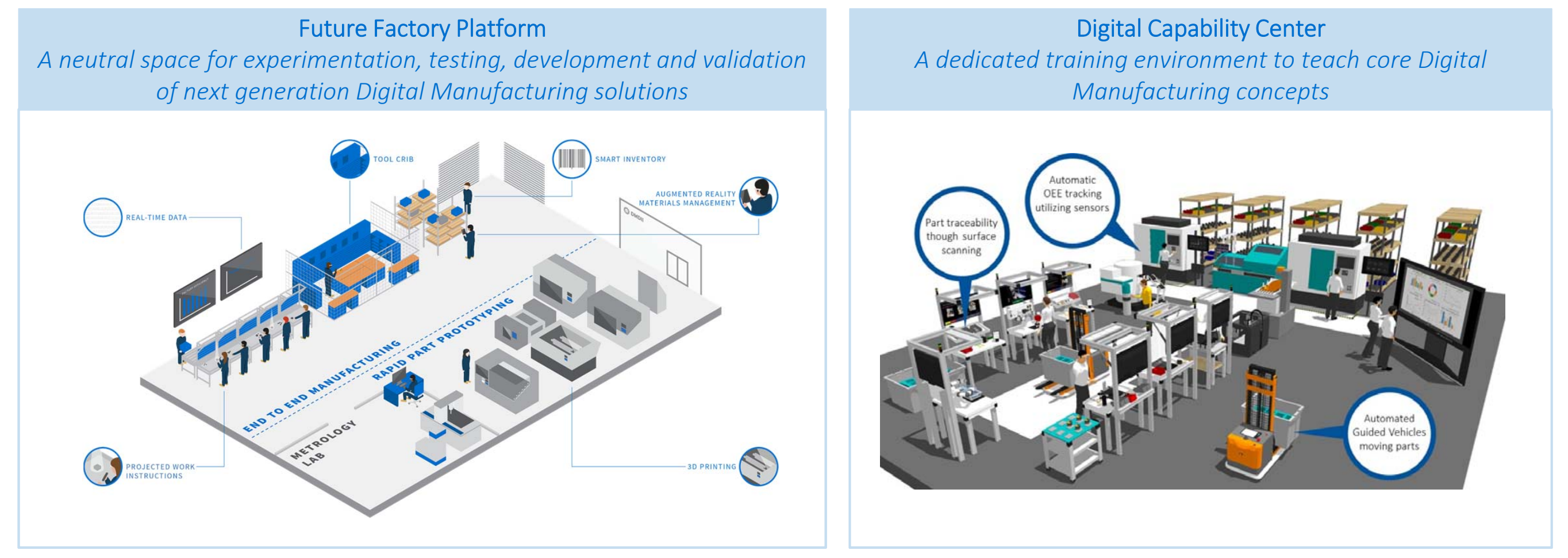

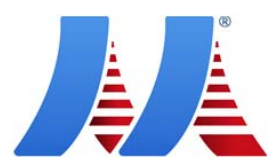




\section{4) Each Institute is operated by an industry-led consortium}

\begin{tabular}{|c|c|}
\hline $\begin{array}{l}\text { Aerospace \& } \\
\text { defense }\end{array}$ & 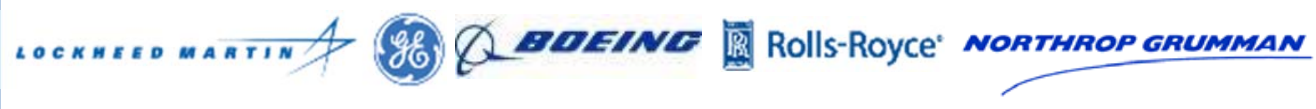 \\
\hline $\begin{array}{l}\text { Industrial } \\
\text { equipment }\end{array}$ & 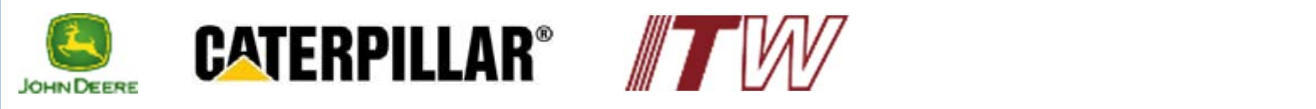 \\
\hline CPG & StanleyBlack\&Decker DURACELL \\
\hline \multicolumn{2}{|l|}{$\begin{array}{l}\text { Chemicals \& } \\
\text { agriculture }\end{array}$} \\
\hline Automotive & feurecia \\
\hline $\begin{array}{l}\text { Pharma \& } \\
\text { medical } \\
\text { products }\end{array}$ & Gohnson affohnson \\
\hline $\begin{array}{l}\text { High tech \& } \\
\text { telecom }\end{array}$ & SIEMENS Microsoft A AUTODESK. \\
\hline Services & $\begin{array}{l}\text { McKinsey } \\
\text { \&Company }\end{array}$ \\
\hline
\end{tabular}

Small to Mid-sized Manufacturers

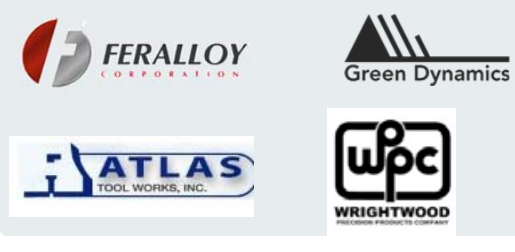

High growth Startups + Technology Providers

\section{4므으 UP^SKILL}

[.

Universities + Community Colleges

\begin{tabular}{|c|c|}
\hline Georg & $\begin{array}{l}\text { Resegrch } \\
\text { Donsiticuthe }\end{array}$ \\
\hline II I L L L I I N O O I I & \\
\hline
\end{tabular}

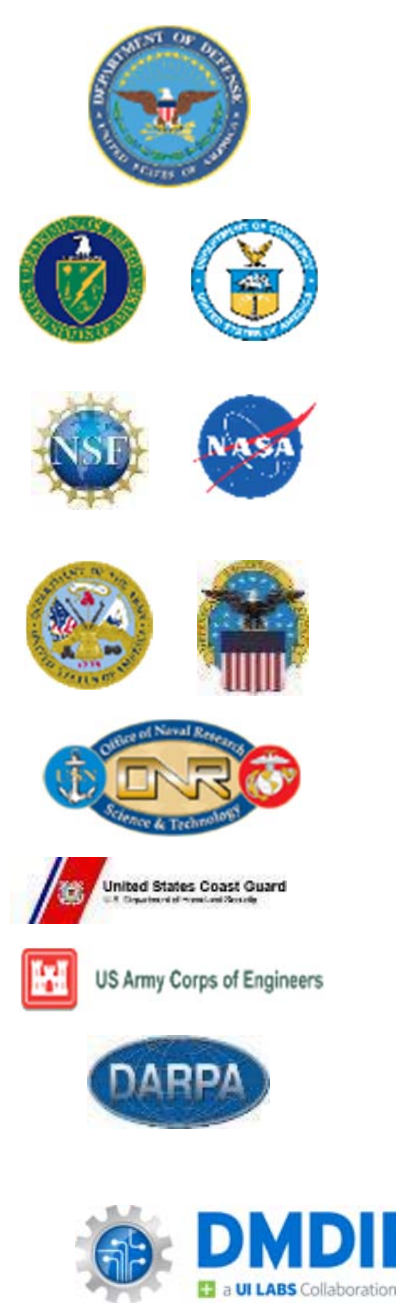




\section{5) Federal start-up funding for each Institute must catalyze at least $100 \%$ co-investment}

$\$ 70,000,000$

Digital Manufacturing Innovation Institute

(-) DMDII

\section{Funding}

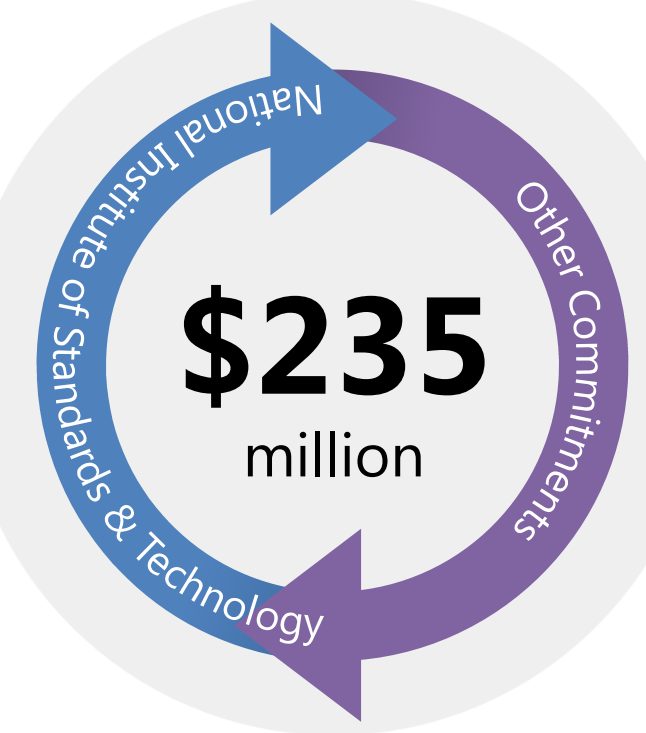

$\$ 165,000,000$

Other Commitments

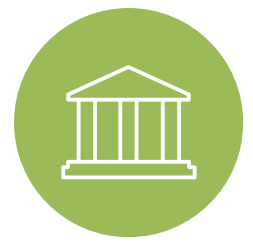

DMDII is funded by a five year $\$ 70,000,000$ cooperative agreement from the federal government and leverages $>\$ 180,000,000$ in other commitments. 


\section{6) Each Institute works on the industry priorities and big challenges only solvable by collaboration}

\section{THEME}

\section{Move Manufacturing to the Left}

Inform conceptualization and design phases with relevant, data-driven insights from across the entire product lifecycle. Ultimately part and product-related data of all kinds should move bidirectionally across the digital thread from concept to end-of-life.

\section{Integrate, Reduce-to-Practice to Drive RO}

Connect the dots of digital manufacturing, discover the remaining impediments to adoption and work through them. Integrate portfolio project outcomes plus emerging commercial technologies in DMDII's Future Factory sandbox as well as in a digital twin pilot involving a member manufacturer's operational environment.

Deliver Promise of Digital Thread \& Digital Twin

Connect previous MBD/MBE/Digital Twin work with new project calls, workshops and pilots to build on the aggregate learnings. The proposed initiatives strive to reduce the technology to practice with pragmatic solutions that are inspired by real-world constraints represented through pilots and member feedback.

\section{Protect America's Growing Digital Manufacturing Advantage}

Digital Manufacturing tech increases the sector's attack surface and simultaneously makes it an even more attractive target as the U.S. builds competitive economic advantage. A key focus is cyber-hardening small-to-medium-sized manufacturers (SMMs), which represent o $90 \%+$ of U.S. manufactured GDP.

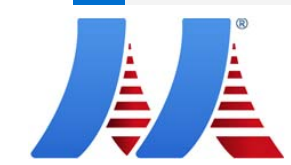

\section{OBJECTIVES}

- Pilot: "Day in the life of CAD"

- Workshop/project: Real-time CAD feedback

- Transitions: facilitate select project commercialization

- Pilot: Factory digital twin in member operations

- Workshop: Sensor ROI \& Marketplace

- Integrations: $17+$ projects $\& 3^{\text {rd }}$ party solutions

Pilot: Supply chain design and digital twin

- Workshop/playbook: Pragmatic model-based-definition

- Workshop/pilot: Blockchain for supply chain use cases

- Cyber Security Hub: Work with DoD to establish**

- SW Tool: SMM cyber assessment \& mitigation

- Training program: SMM cyber security basics** 


\section{7) Each Institute manages a balanced portfolio of real projects for}

\section{industry}

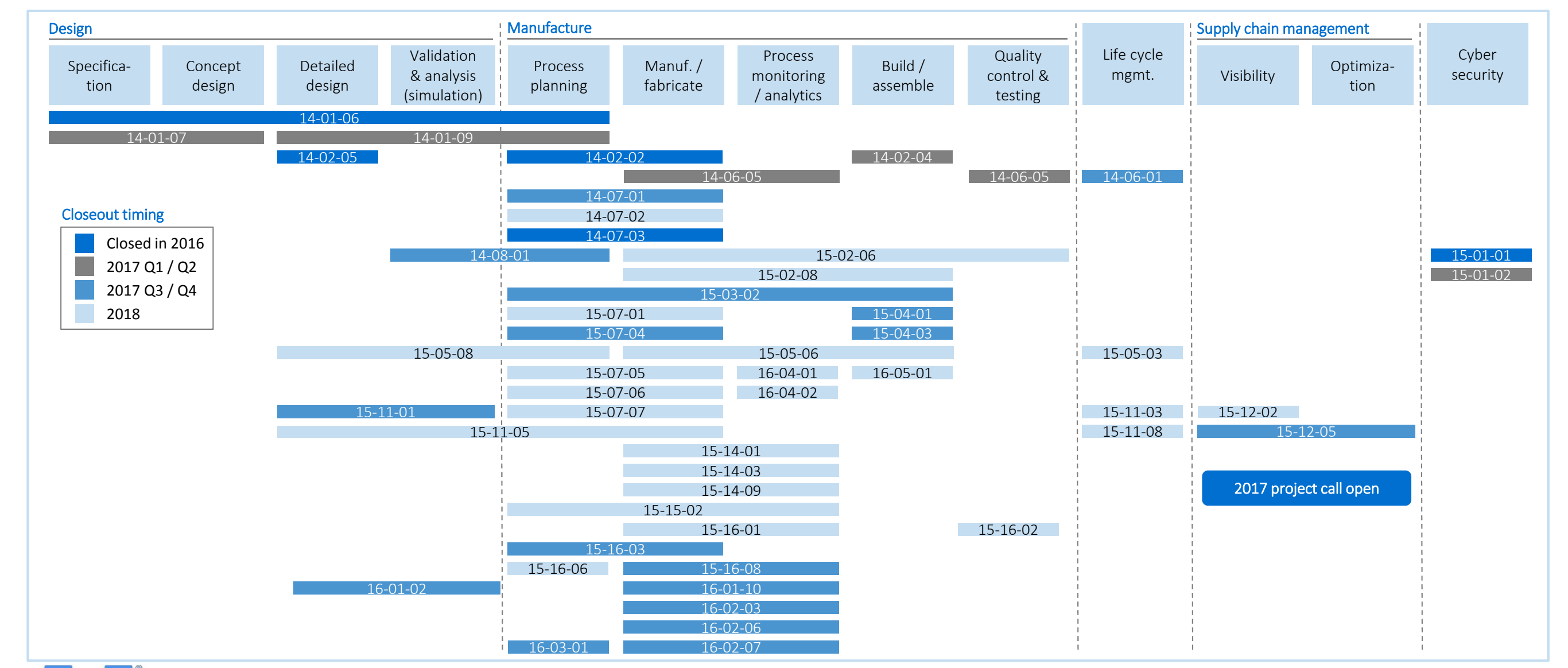

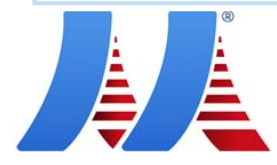




\section{8) Each Institute addresses the skills gap on education and workforce skills for their technology space}

In collaboration with SUNY at Buffalo, DMDII has developed a MASSIVE OPEN ONLINE COURSE on digital manufacturing \& design, demonstrating how these tools can be used throughout the product lifecycle.
Through the MEP Institute Embed Program, DMDII is developing ASSESSMENT TOOLS AND USE CASES TO

SUPPORT SMMs in the adoption of new technologies and processes.

DMDII is also building the DIGITAL MANUFACTURING COMMONS to extend the reach and scale of content, applications, and services to SMMs across the U.S.
Working with Manpower Group, DMDII has identified 165 NEXT-GEN JOB PROFILES/ROLES IN MANUFACTURING that will be created or transformed by the introduction of digital technology in the industry. This body of work defines the skills required to execute defined job classifications.

Online Courses

Jobs Taxonomy WFD
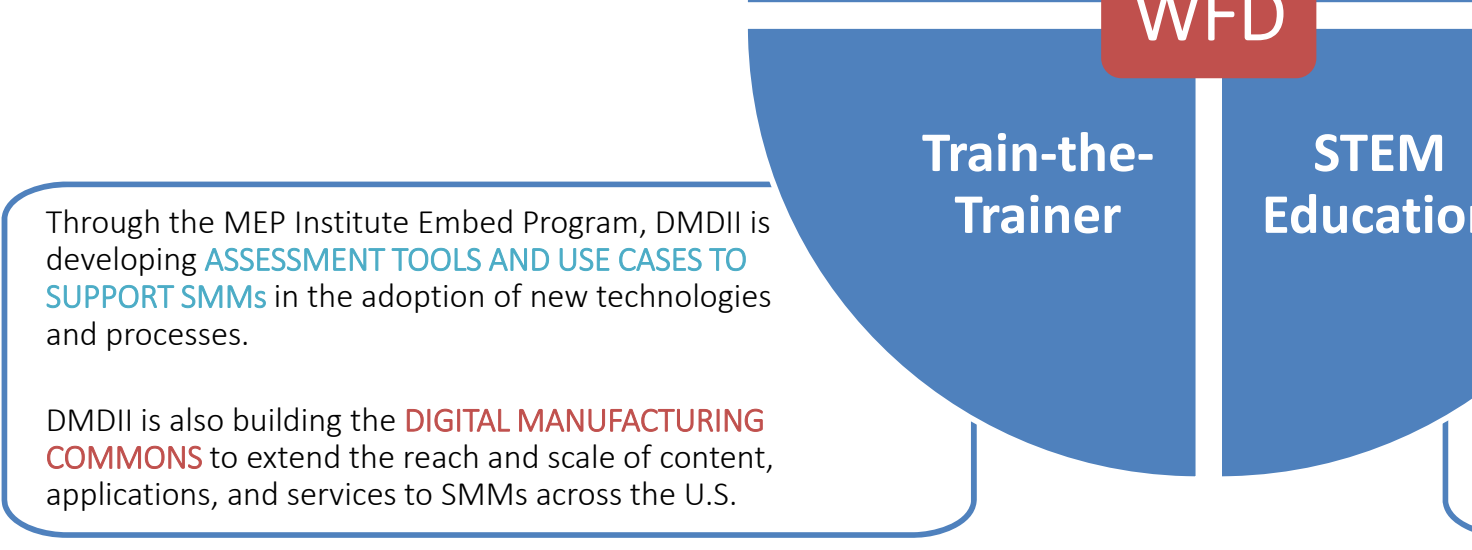

Through our DIGITAL DAYS program, DMDII hosts middle and high schools for an afternoon of educational STEM programming and manufacturing career awareness. 


\section{Agenda}

- Manufacturing USA Overview

- How an Institute Works - DMDII

- Delivering Value: 2017 Results Highlights

- Example PHM Projects 


\section{Impact to U.S. Innovation Ecosystem - Membership}

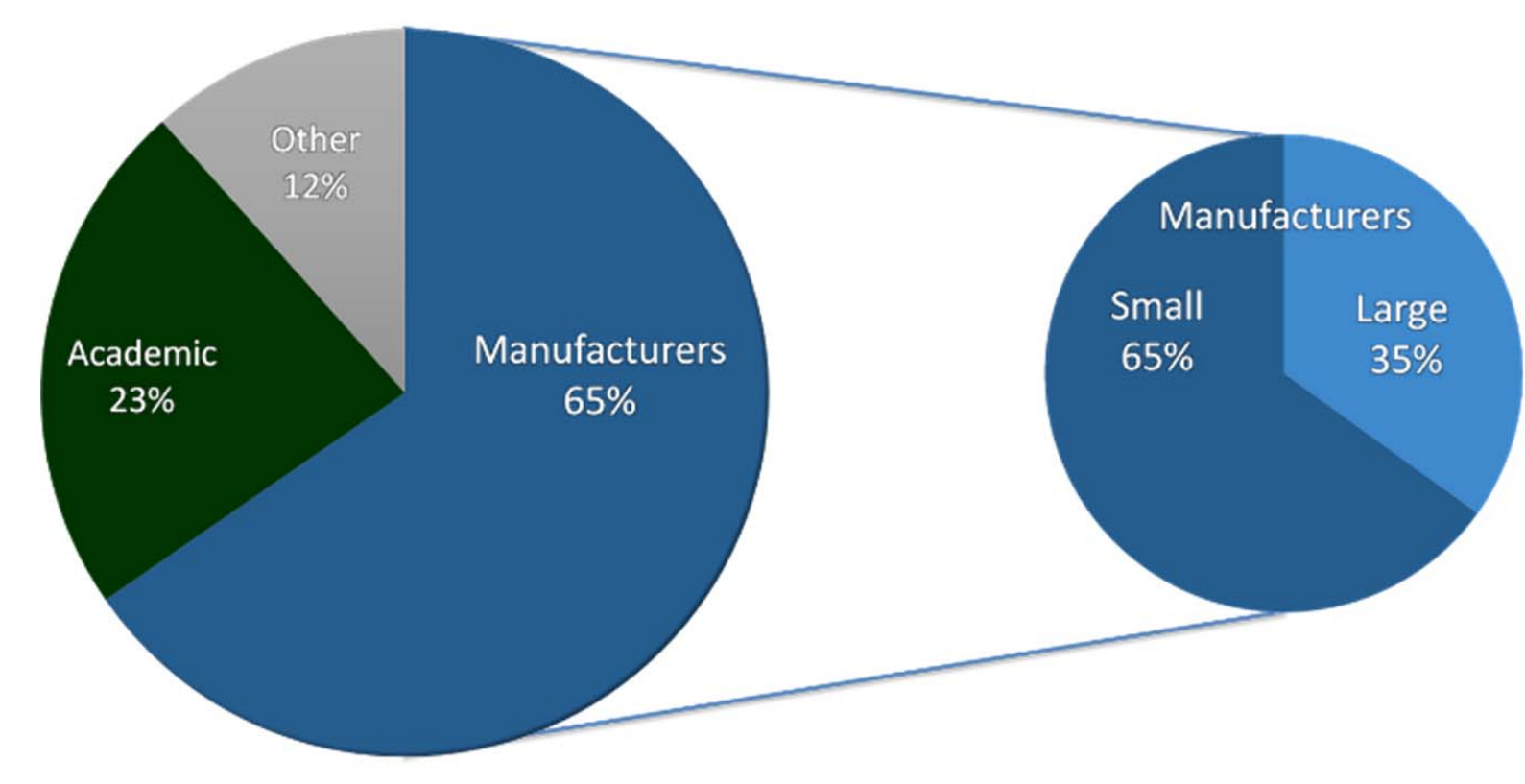

1,291 members (FY 2017)

$+50 \%$ increase in membership over 2016

$65 \%$ from industry

o $65 \%$ are small and medium-sized manufacturers

297 universities, community colleges, and other academic institutions

150 federal, state, and local government agencies, federal laboratories, and not-for-profits Membership breakdown of 12 institutes in FY 2017 


\section{Leveraging Co-Investments}

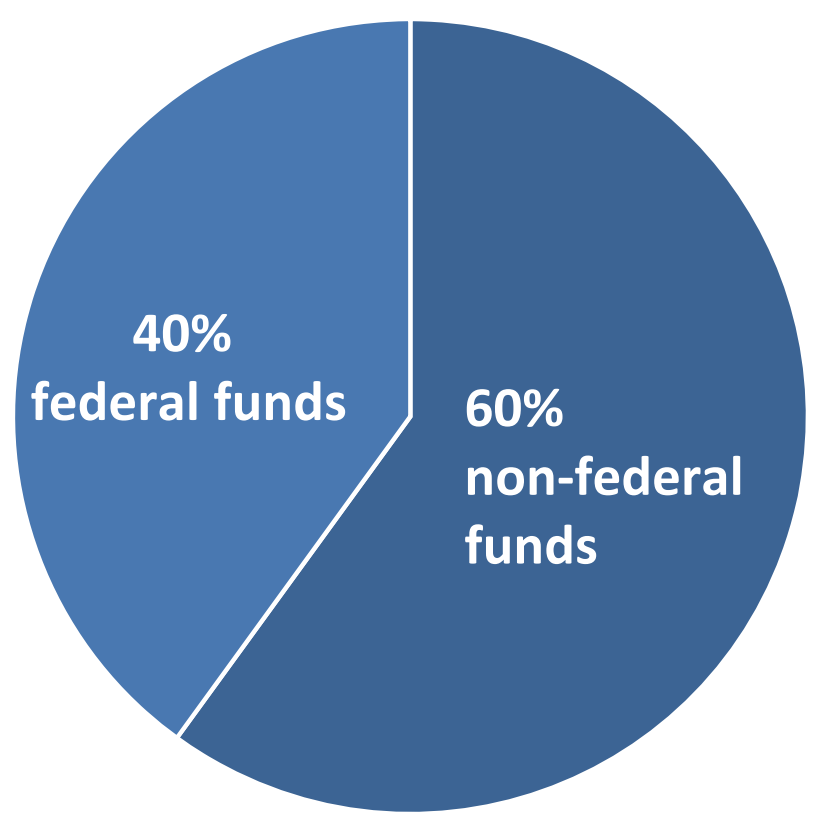

\section{More than 1.5 to 1 investment match $(\mathrm{Fr} 2017)$}

\section{$\$ 298,500,00$ in total institute expenditures}

- $60 \%$ of institute support came from non-federal matching funds

- $40 \%$ came from federal program funds

Expenditures funded all aspects of institute operation (e.g. technology advancement projects, education and workforce training efforts, and capital equipment) 


\section{Technology Advancement}

While many technology R\&D

projects can take several years

to conclude, the high level of

participation by industry and

the progress in meeting

technical objectives are early

indicators of success.

\section{Major \\ Collaborative \\ R\&D Projects \\ FY 2017}

百 


\section{Agenda}

- Manufacturing USA Overview

- How an Institute Works - DMDII

- Delivering Value: 2017 Results Highlights

- Example PHM Projects

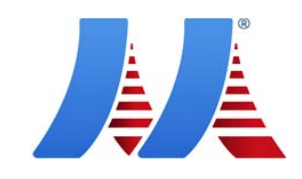




\section{DMDII Project Portfolio includes PHM}

\section{Monitoring}

Increasing accessibility to manufacturing data

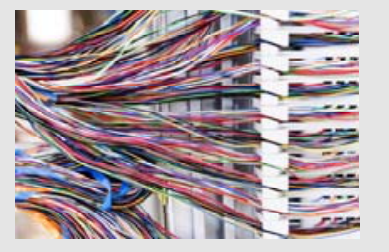

16-02-03: Reconfigurable Retrofit Kit for Legacy Machines - Non-invasive sensors application to enable data capture on older machines

\section{Diagnostics}

Enabling advanced analytics on manufacturing data

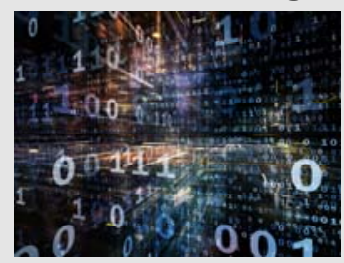

15-14-01: Cloud-Enabled Machines with Data-Driven Intelligence - Framework for cloud-based online machine and process monitoring, diagnosis, and prognosis

\section{Prognostics}

Making decisions based on data to increase

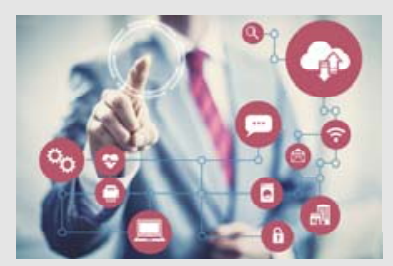

16-04-01: Achieving Smart Factory through Predictive Dynamic Scheduling - Improve operations by combining MES metrics like OEE with predictive maintenance analytics 


\section{6-02-03: Retrofit Kit for Legacy Machine Sensing in Secure Data Environments}

Development of a reconfigurable retrofit kit for legacy equipment that provides significant flexibility and state-of-the-art network security

\section{INDUSTRY CHALLENGE}

Production optimization efforts for manufacturing organizations with primarily legacy equipment platforms are severely hindered by limited capabilities for low cost, user-configurable machine connectivity and in situ machine sensing. This project will address this technological gap by developing an open-platform, reconfigurable retrofit kit that provides for scalability of cost of ownership and enables user-driven selection of sensing capabilities.

\section{PROJECT SOLUTION AND OUTCOMES}

The project will assemble a network-secured, scalable retrofit kit that provides for integration of highly flexible machine sensing for a range of production environments encompassing both legacy and modern machine equipment. A unique aspect is that it will be built upon an industrially hardened Layer-3 compatible communications platform for isolating machine tools from network intrusion and will facilitate highly reconfigurable sensing using both wired and wireless communications protocols. This will enable manufacturers to seamlessly design and implement data sensing schemes to accommodate continuously evolving data measurement needs.

Georgia Tech, Mazak, ITAMCO, Caterpillar

\section{POTENTIAL IMPACT}

» The retrofit kit will provide the needed transitional technology for realizing ubiquitous and network secured sensing for legacy equipment.

" Data accessibility will further enable US manufacturers to leverage advanced analytics to strengthen competitiveness compared to more nascent, predominantly modern production capabilities worldwide

"Open-source, low-cost sensing for industrial platforms can be significant for user-driven process improvement efforts

Machine Status

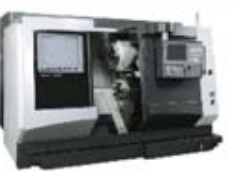

Mactine name: Mactire wild Mors information

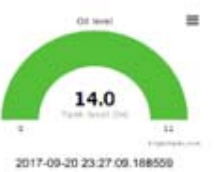

mitar
OkumaLU3000ex

$\infty 1$

specs

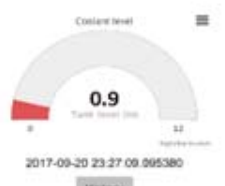

$\operatorname{mos}$

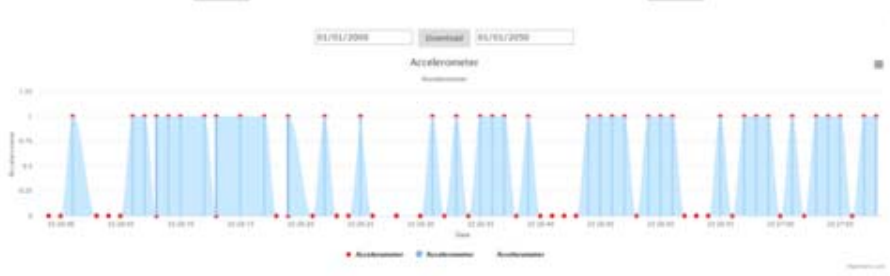




\section{5-14-01: Cloud-Enabled Machines with Data-Driven Intelligence}

Framework for cloud-based online machine and process monitoring, diagnosis, and prognosis

\section{INDUSTRY CHALLENGE}

One of the primary problems faced by both small and medium sized manufacturers and large original equipment manufacturers is how to develop new machines with intelligence as well as retrofit legacy machines with intelligence so that in-process, remote monitoring, diagnosis, prognosis, and self-correction can be automatically performed.

\section{PROJECT SOLUTION AND OUTCOMES}

" an interoperable data acquisition system that consists of a wireless sensing system, Predix ${ }^{\mathrm{TM}}$ Machine software, Predix ${ }^{\mathrm{TM}}$ powered gateway device, and a scalable on-premise private cloud platform

" a container-based high performance cloud computing platform that is integrated with the on-premise private cloud for processing real-time data streams, executing parallel machine learning algorithms, generating big data analytics, and visualizing data

" a set of experimentally tested algorithms that enables data-driven intelligence for online spindle diagnosis and prognosis in both legacy machines and general purpose CNC machines, executable on a hybrid cloud computing platform

Penn State University, GE Global Research, Microsoft, Case Western Reserve University

\section{POTENTIAL IMPACT}

" Ubiquitous and instant remote access to near real-time data without spatial constraints

"Secure and high volume data storage along with scalable, high performance computing.

"Big data analytics enabled by parallel and distributed computing, data mining and machine learning algorithms can be developed that enable manufacturers to process and manage massive data streams on a cloudbased computing platform.

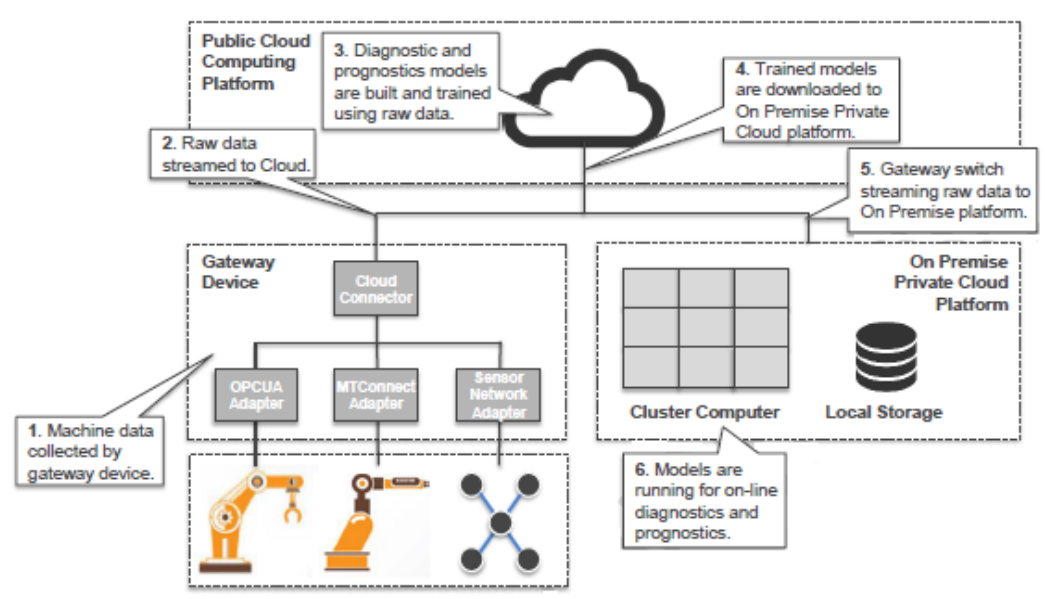




\section{6-04-01: Achieving Smart Factory through Predictive Dynamic Scheduling}

Improve manufacturing operations by combining traditional MES technology metrics like OEE with in-depth predictive maintenance analytics

\section{INDUSTRY CHALLENGE}

" Current MES/OEE monitoring systems do not provide drill-down capabilities that enable end-users to investigate the condition/health of the machine so that appropriate measures can be performed to non-performing units

» Dynamic scheduling systems allow manual inputs or time-based inputs (preventive maintenance schedules) but they do not consider the actual condition/health of the machine.

" Actions based solely on machine health metrics are difficult to justify unless they are tied to factory performance metrics such as OEE and the predictive nature of these solutions are not harnessed to its full potential unless they affect actual maintenance schedules.

\section{PROJECT SOLUTION AND OUTCOMES}

» The customization of predictive health monitoring system and prognostics algorithms for accurate machine health estimation and prediction

" A systematic methodology for synthesizing system-level factory information and machine-level predictive health information into a Markov Decision Process model for predictive maintenance opportunity window estimation

"A new paradigm for maintenance scheduling that utilizes real-time health condition of machines, predictive analytics of future performance and remaining useful life, and system production information (e.g., buffer contents, short-term production requirement).

Forcam Inc., Predictronics Corp., Lockheed Martin, Northeastern University

\section{POTENTIAL IMPACT}

" Ubiquitous and instant remote access to near real-time data without spatial constraints

"Secure and high volume data storage along with scalable, high performance computing.

" Big data analytics enabled by parallel and distributed computing, data mining and machine learning algorithms can be developed that enable manufacturers to process and manage massive data streams on a cloudbased computing platform.

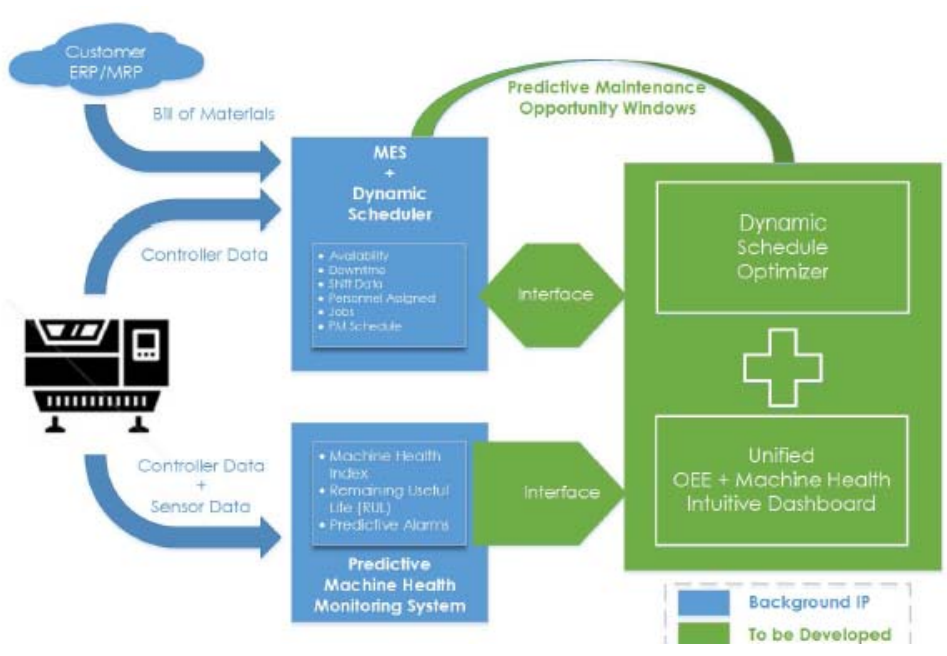




\section{Together We Are Securing America's Future}

\section{Making an Impact}

- 14 institutes developing new manufacturing techniques

- 300 ongoing major collaborative R\&D projects

- 200,000 people trained in advanced manufacturing

- \$1B federal investment matched by over \$2B non-federal funds

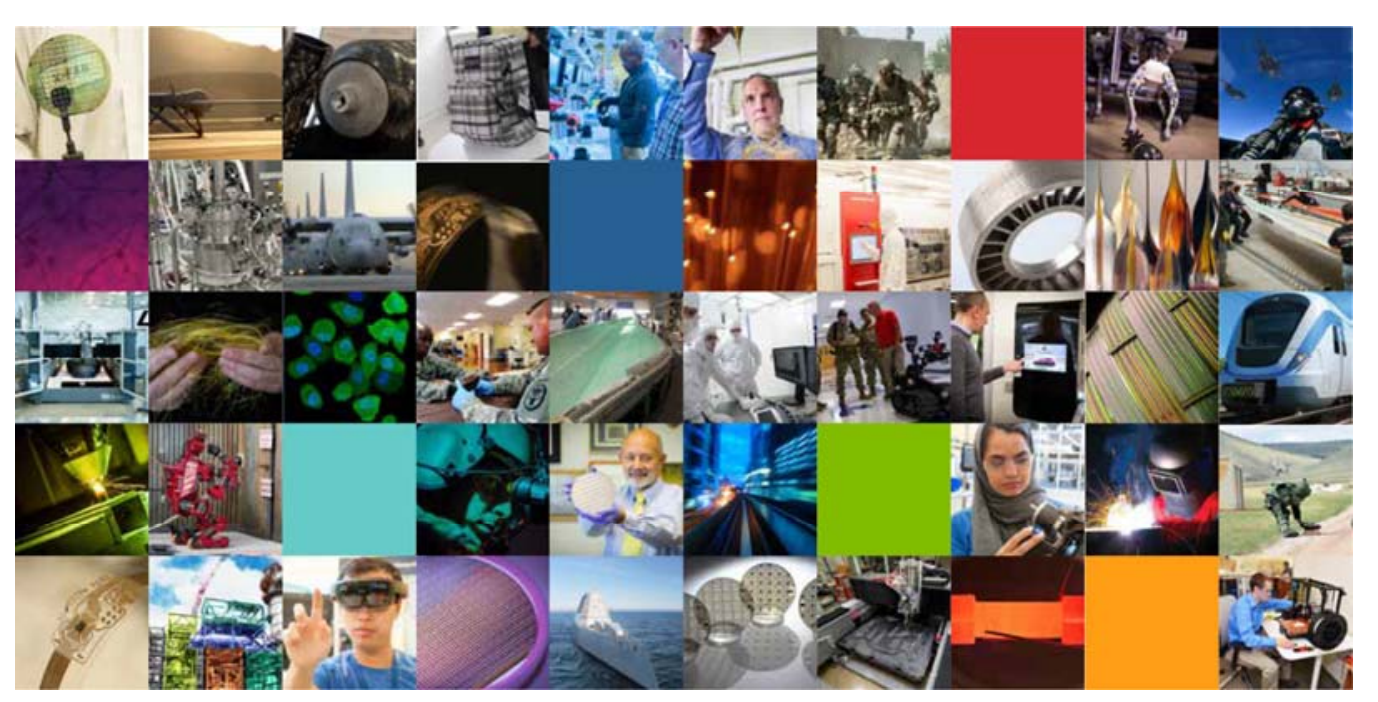




\section{Thank you}

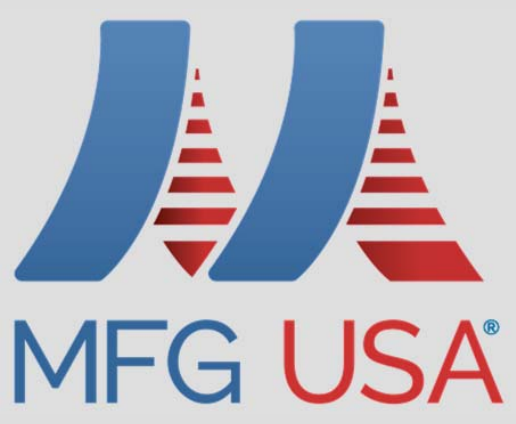

www. ManufacturingUSA. com
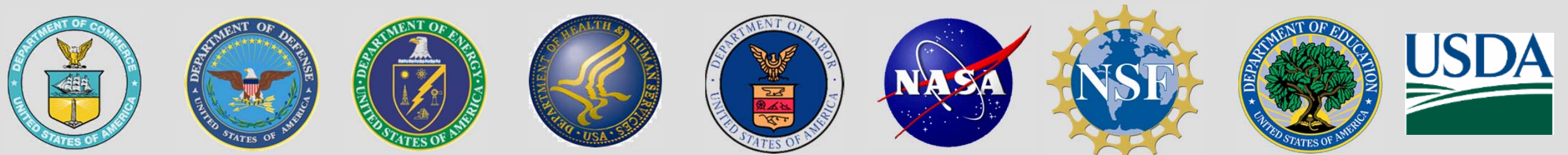

ARM ADVANCED ROBOTICS
FOR MANUFACTURING 
NIST Smart Manufacturing Programs: Driving Innovation and Reducing Risks of Adoption of New Technologies

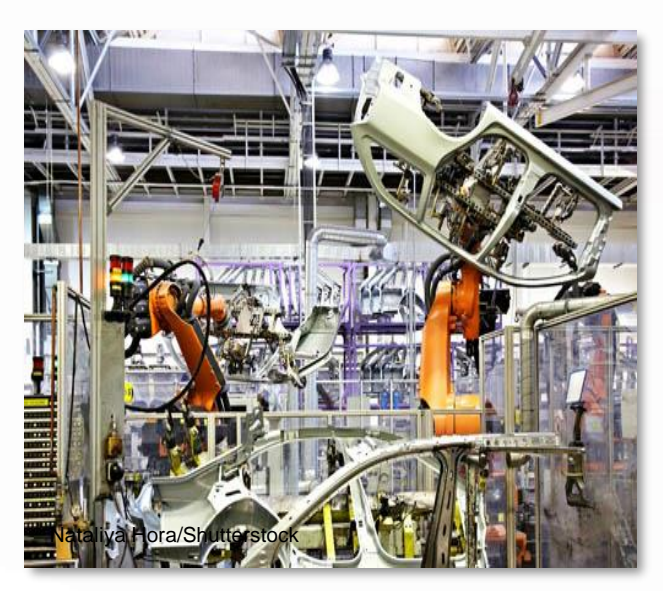

\author{
Albert Wavering \\ National Institute of Standards and Technology \\ U.S. Department of Commerce
}




\section{NIST and Manufacturing}

"It is therefore the unanimous opinion of your committee that no more essential aid could be given to manufacturing [...] than by the establishment of the [National Bureau of Standards]."

House Committee report, May 1900

NIST Mission: To promote U.S. innovation and industrial competitiveness by advancing measurement science, standards, and technology in ways that enhance economic security and improve our quality of life.
A partner to US manufacturers for more than a century, NIST helps the nation's manufacturers to invent, innovate, and create through:

- Measurement science - manufacturers and technology providers use NIST test methods, measurement tools, performance measures, and scientific data every day

- Advanced materials - NIST is building a materials infrastructure to accelerate the timeline from design to deployment of new materials

- Standards development - NIST provides the scientific and technical basis for voluntary consensus codes and standards

- Partnerships - collaborations with the private sector and academic organizations help advance and disseminate research and support US manufacturers 


\section{NIST Helps Drive Innovation and Reduce}

Risks of Adoption of Emerging/Disruptive Manufacturing Technologies

...by contributing to standards that provide a common language and test methods that technology suppliers and users can use to assess and communicate technical capabilities and performance.
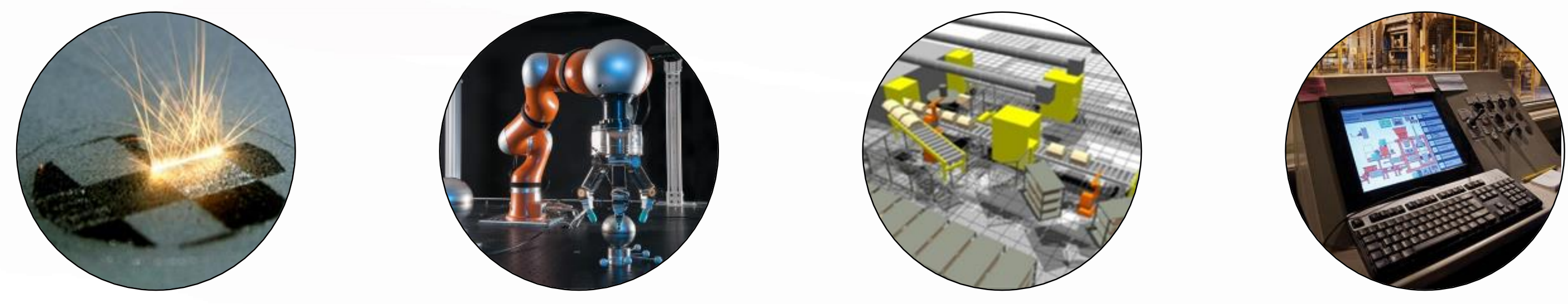


\section{Driving Innovation and Reducing Risks of Technology Adoption Through Measurements and Standards}

Testbeds

Industry/Academia/Government Consortia, Standards Organizations

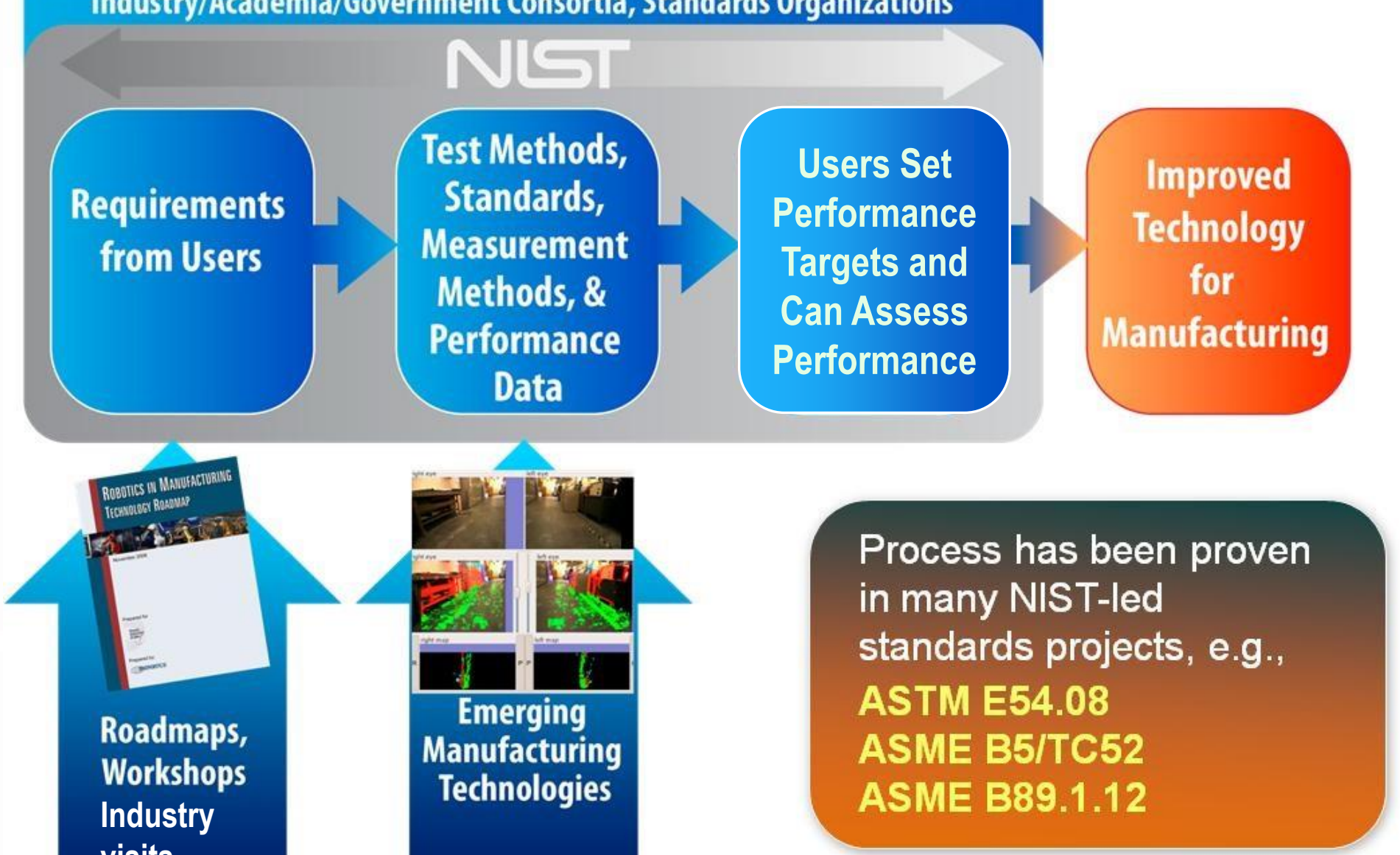




\section{Example: 3D Sensor Systems}
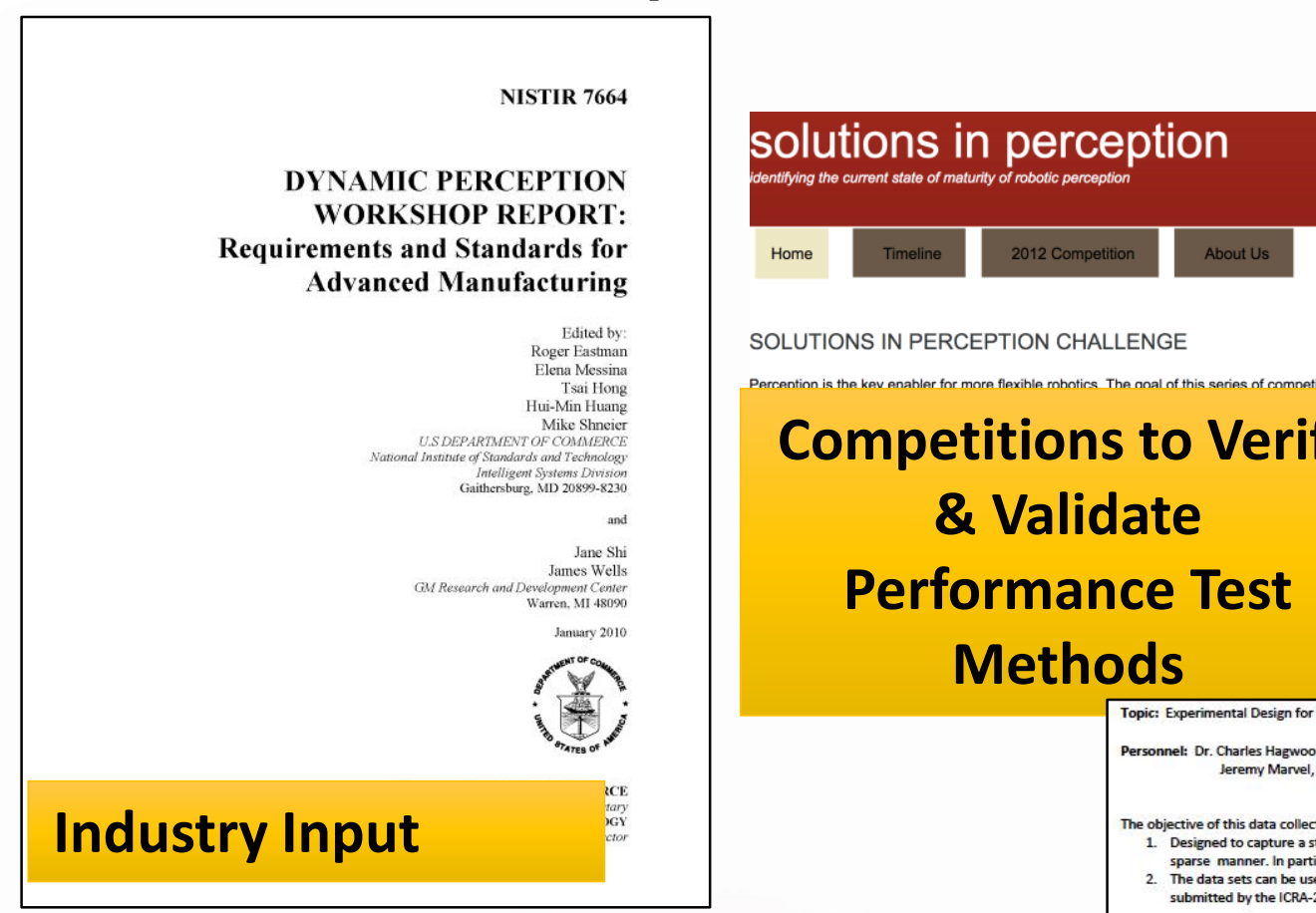

SOLUTIONS IN PERCEPTION CHALLENGE

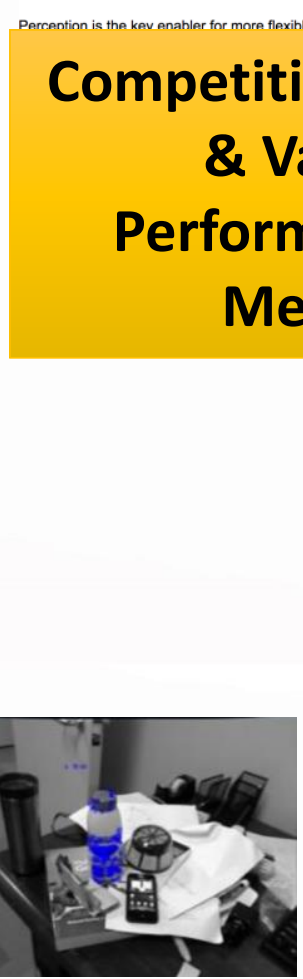

Emerging Technologies

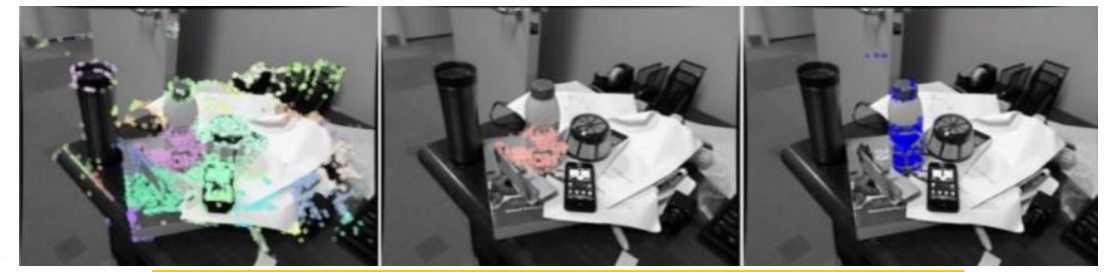

8

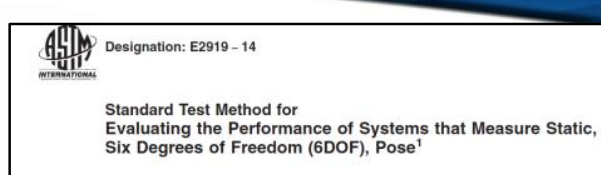
Six Degrees of Freedom (6DOF), Pose'

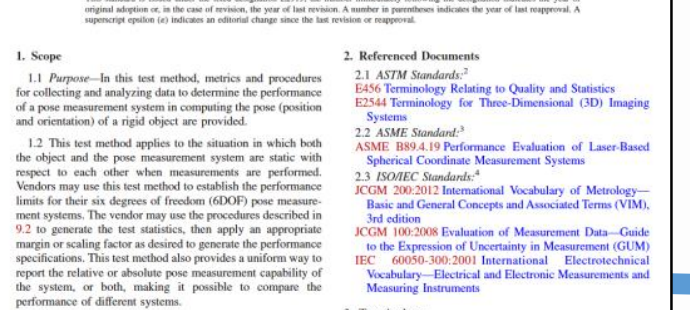

Sponsors

Willow Garage

NIST

IEEE
Robotics
SOCH

Roobotes and Automation
socoly

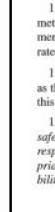

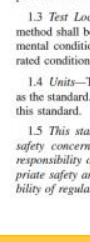

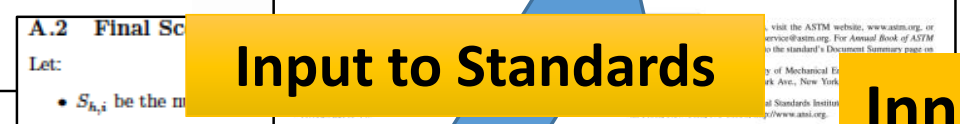
- $S_{m, i}$ be the the number of hisses (1.e., ralise-ne - $S_{n, i}$ be the number of noise (i.e., false - $S_{r_{i}}$ be the rotation score in frame 1. - $S_{t, i}$ be the translation soore in frin - $s_{-, i}$ be the negative scores in fir. - $N$ be the total number of frames from $=$ Runs combinect - $O$ be the total number of detectable objects in all frames and Runs combines Then,

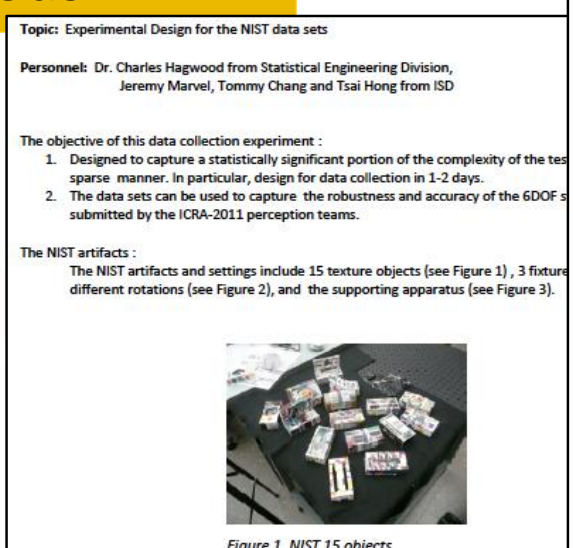

Final Scoure $=\frac{1}{2} \sum_{i=1}^{N} \max \left(\left(S_{h, i}-0.5 S_{m, i}-S_{n, i}+\frac{S_{r, i}+S_{t, i}}{2}\right), 0\right)$ $\equiv \frac{1}{2} \sum_{i=1}^{N}\left(S_{h, i}-0.5 S_{m, i}-S_{n, i}+\left|S_{-, i}\right|+\frac{S_{r, i}+S_{L, i}}{2}\right)$
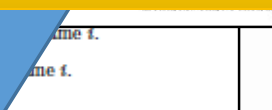

Hic Peraten (TP)

Draft Metrics \& Test Methods
ASTM E57 3D Sensor Systems

\section{Innovation through}

- Standardized methods of measuring performance to reduce risk of adopting wrong solution.

- Metrics to guide design improvement, with inclusion of manufacturing-relevant objects by NIST. 
Smart Manufacturing:

The synthesis of advanced manufacturing capabilities and digital technologies to produce highly customizable products faster, cheaper, better, and greener

NIST Smart Manufacturing Program Areas:

Additive Manufacturing

Robotic Systems
Manufacturing System Design and Analysis

Manufacturing Operations Planning and Control 


\section{Measurement Science for Additive Manufacturing}

Metal Additive Manufacturing: Building metal parts by adding layer upon layer; like 3D printers, but with metals

-What are important measurements for metal Additive Manufacturing materials, and how do you make them?

- How can you get the best performance out of metal Additive Manufacturing processes?

- What measurements are needed to support qualification of metal Additive Manufacturing materials, processes, and parts for critical applications?

- What information is needed to integrate metal Additive Manufacturing into end-to-end manufacturing production?

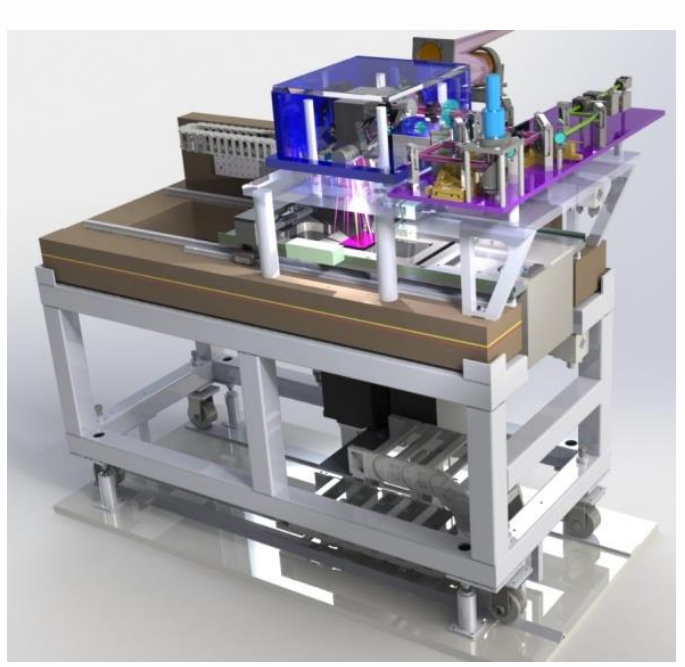

NIST AM Metrology Testbed

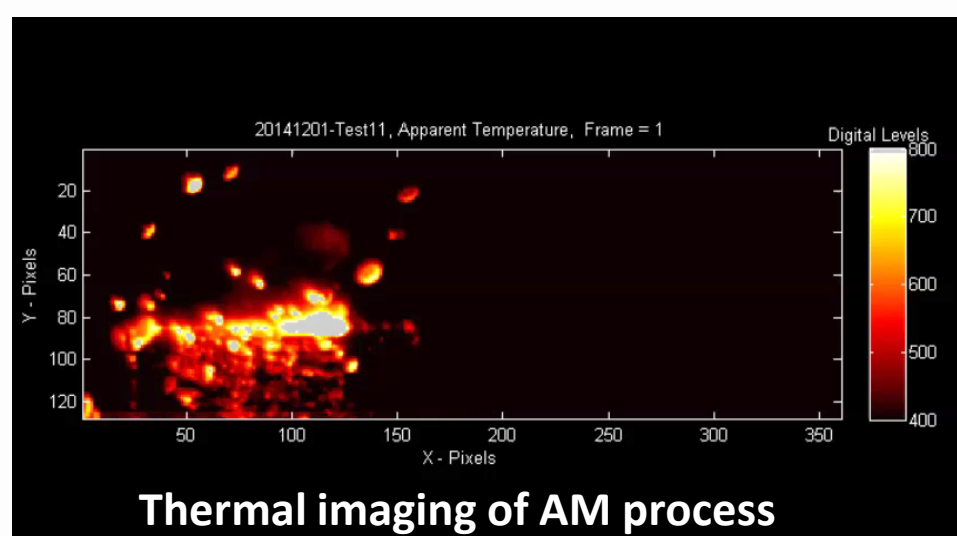




\section{Standards Contributions}

- ASTM Standard Guide for Characterizing Properties of Metal Powders used for AM Processes

- Dimensional - mechanical - thermal - powder bed density - recyclability

- ASTM Standard Guide for Evaluating Mechanical Properties of Metal Materials made via AM Processes

- Mechanical-microstructure - porosity - density - post processing

- Leading ASTM/ISO Joint Working Group for the development of standards for AM test artifacts

- Conducting round robin studies for AM

- Leading new ASME efforts on Product Definition for AM

- Leading new work item in ASTM on Principles of Design Rules

- Prototype Materials Database for AM accessible by public

- Lead development of AM standards strategy within ASTM F42 Executive Committee

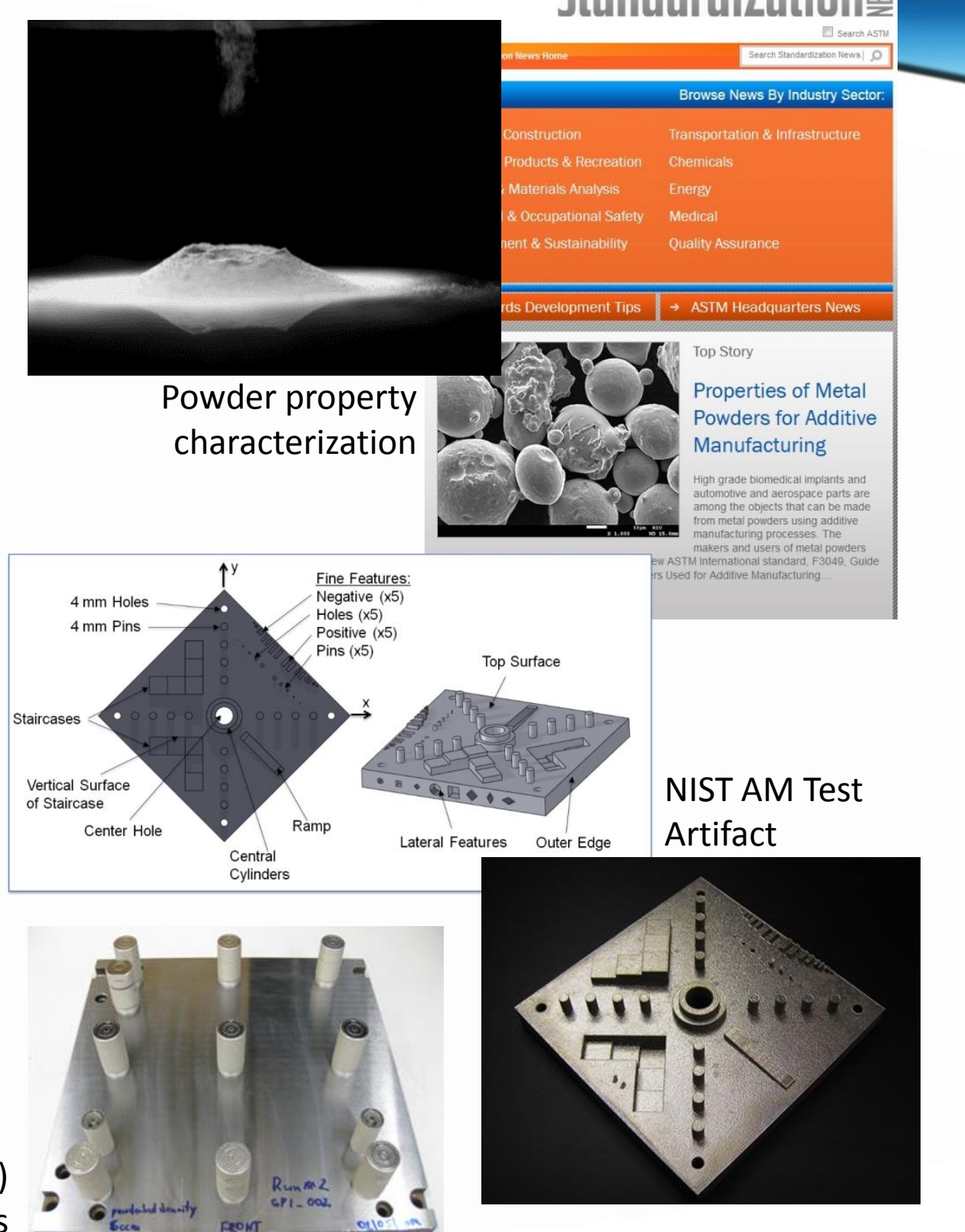




\section{PHM for AM?}

- Initial NIST AM focus is on process understanding, improvement, repeatability, and predictability, rather than PHM aspects

- Early yet to have a good handle on common failure modes for AM

- AM machines are complex, with a number of critical subsystems that each have potential failure modes

- Powder handling/management and spreading/delivery

- Gas flows/build environment control

- Laser/energy control and scanning

- Equipment manufacturers build in sensors,

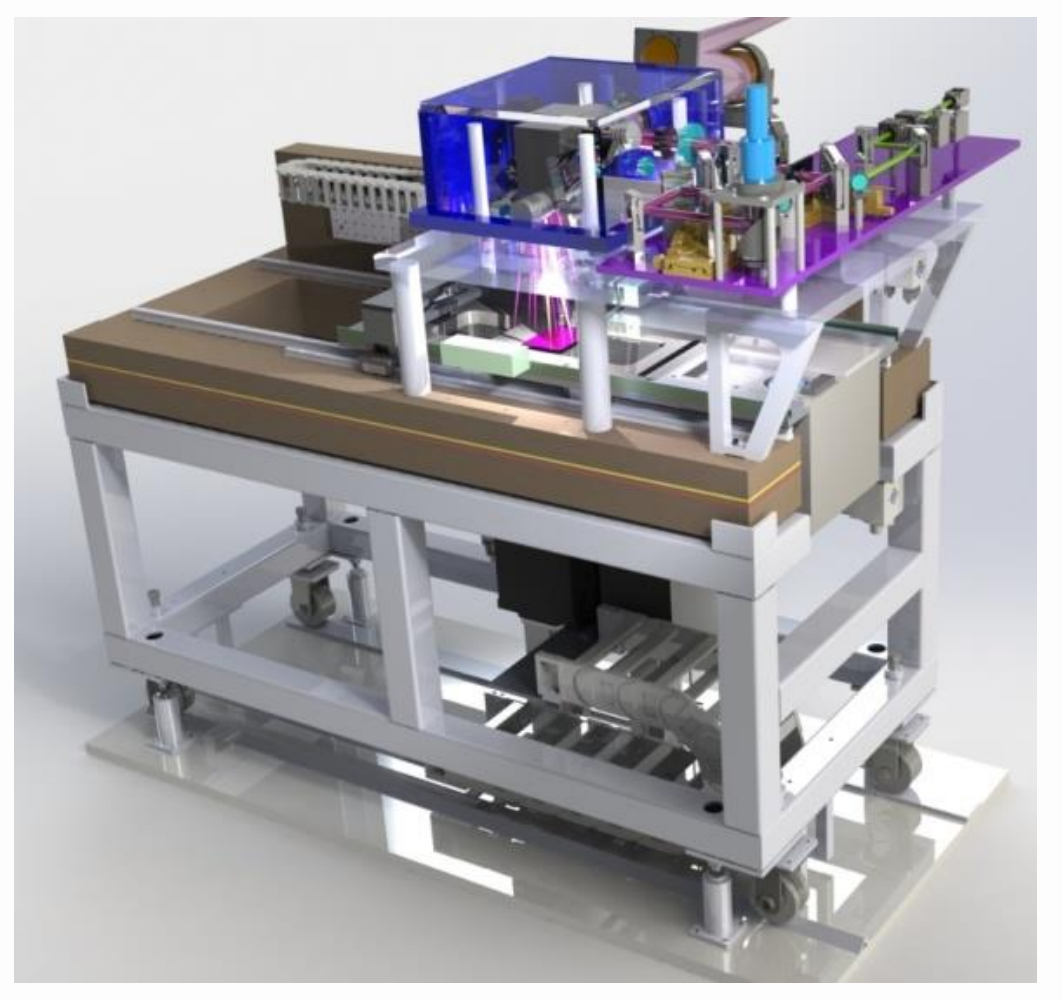
maintenance features, protocols 


\section{Robotic Systems for Smart Manufacturing}

- How can you measure the performance of robotic capabilities such as perception, grasping, manipulation, and mobility?

- How can you measure the effectiveness and safety of new collaborative robotic technologies?

- How can you measure and advance the agility of robotic systems (ease of teaching new tasks, recovering from errors)?

- What standards are needed to more easily integrate robot systems with other factory and control equipment?

- How can we help make robotic technologies more easily adoptable by small and medium-sized manufacturers?
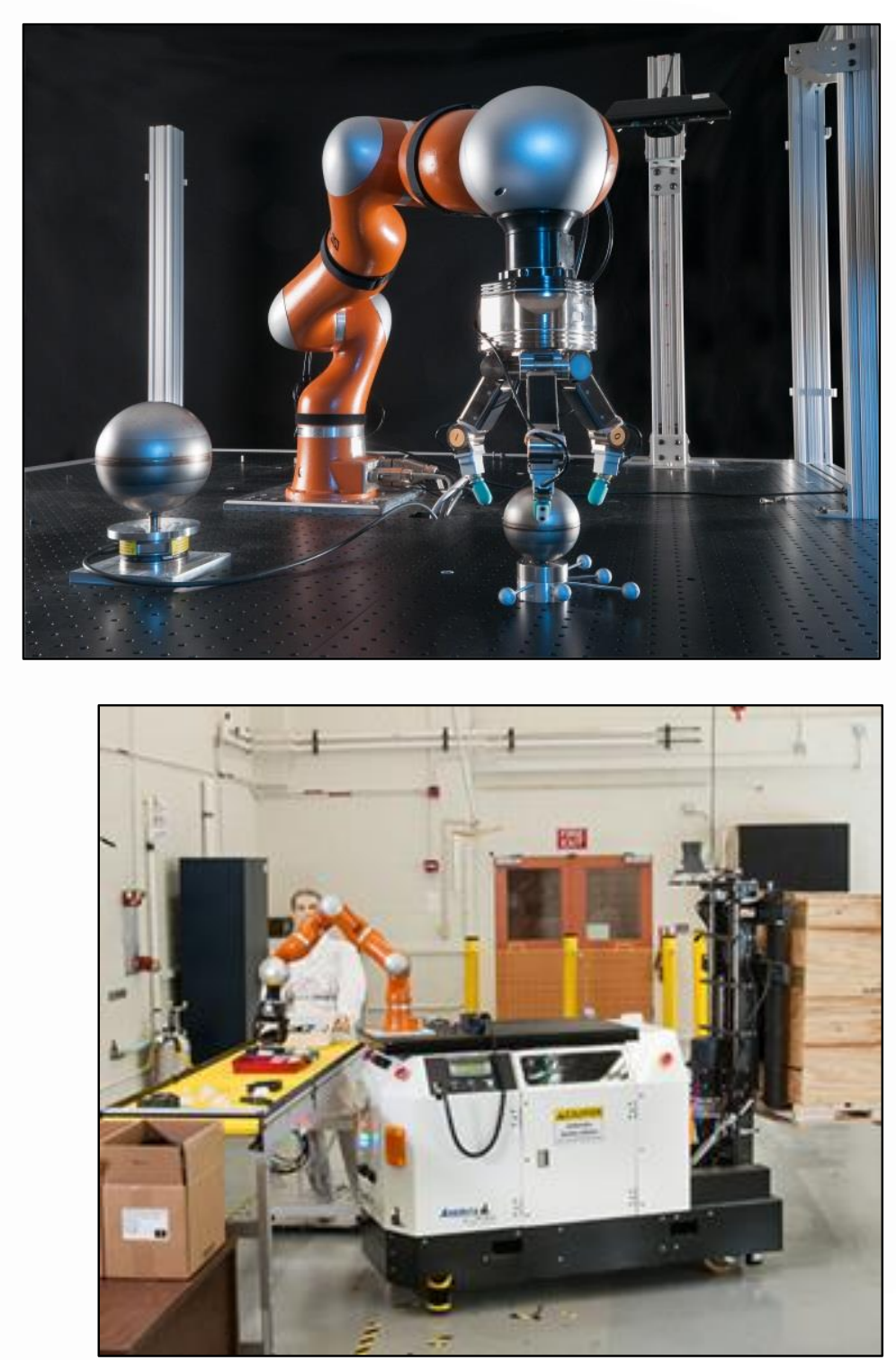


\section{Standards Contributions}

- Performance evaluation of 3D Imaging Systems, including two test methods for 6D pose measurement systems

- Revision of Automatic Guided Vehicle safety standard to enable non-contact obstacle sensing

- New ASTM Committee on performance standards for industrial vehicles

- New international technical specification for collaborative robots safety in industrial settings

- New Robotic Industries Association effort on mobile manipulator safety standards

- New IEEE standard for knowledge representation for robot systems

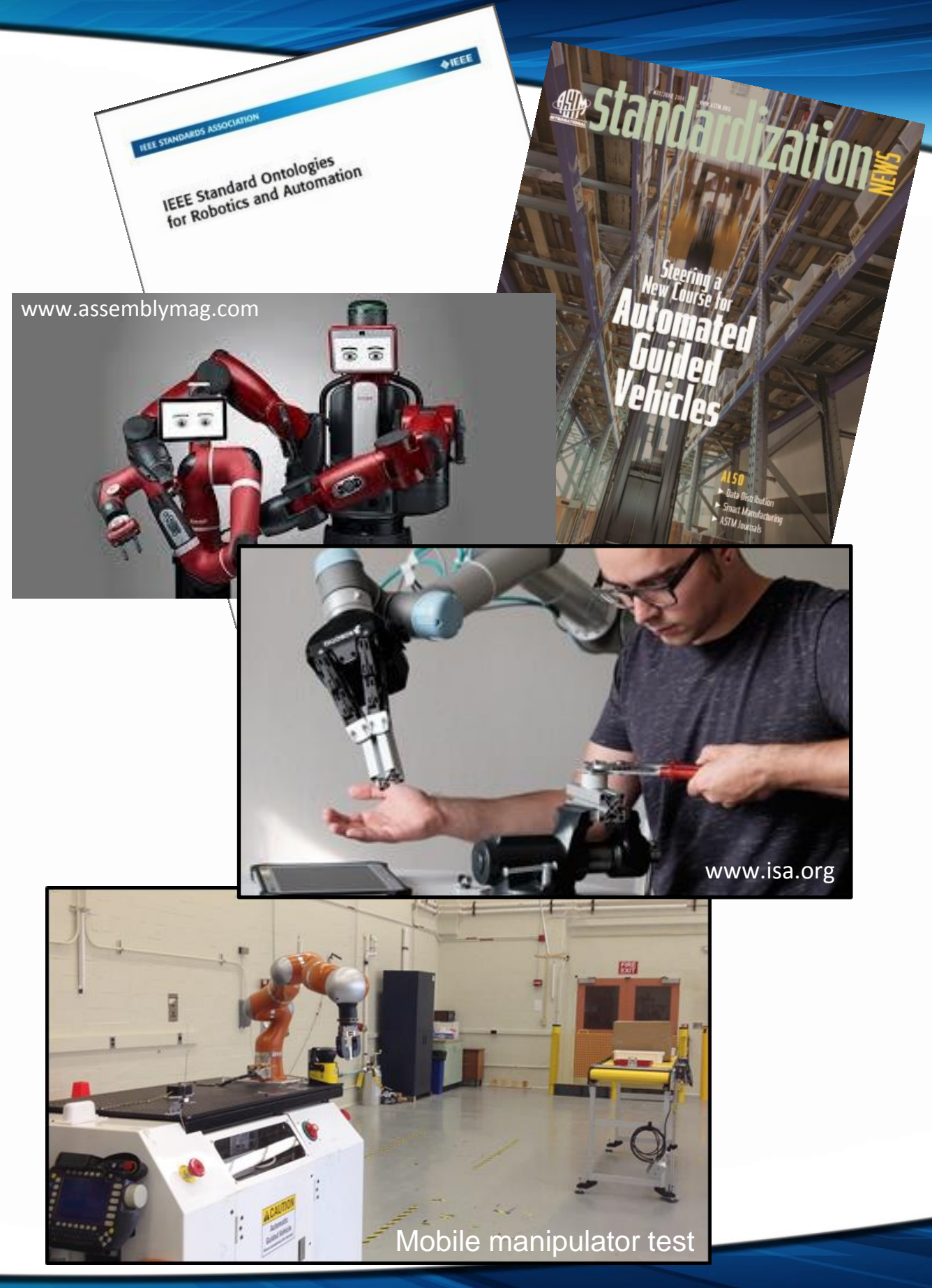




\section{Smart Manufacturing Systems Design and Analysis}

- What standards are needed to support new models of distributed or service-oriented manufacturing?

- What standards are needed to streamline information flow for food manufacturing?

- How can different kinds of modeling software be integrated effectively to support Smart Manufacturing systems?

- What are the best ways to measure the overall performance of manufacturing operations?

- What standards are needed to support data analytics for Smart Manufacturing systems?
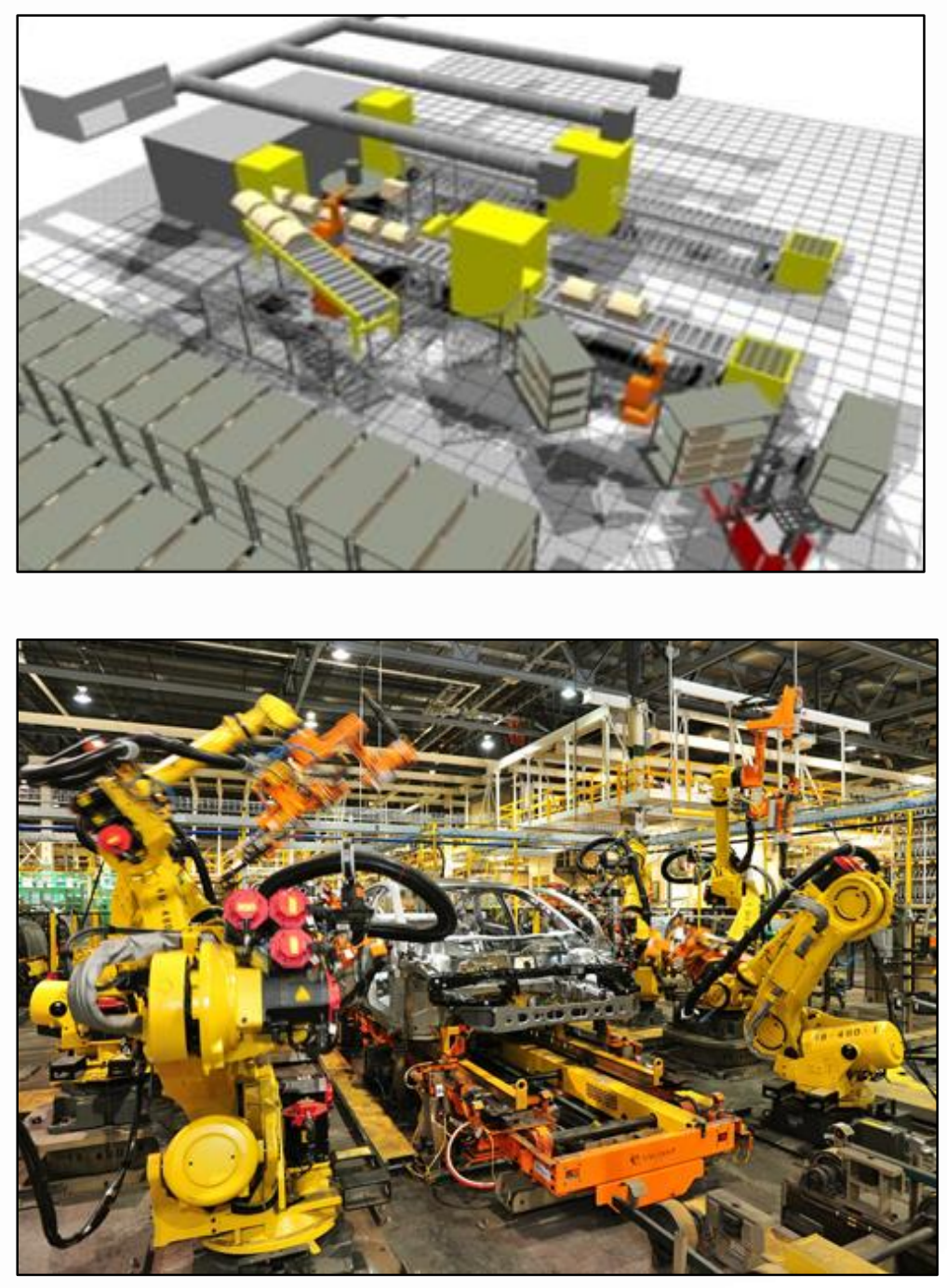


\section{Standards Contributions}

- ASTM E60.13 Guide for Sustainability Characterization of Manufacturing Processes

- Will provide a common basis for sustainability assessment of manufacturing processes.

- Semantic Refinement methodology published as OAGI Working Group specification

- Enables platform-specific manufacturing applications to interoperate based on a common standard (e.g., Mobile vs Enterprise applications)

- Smart manufacturing in the cloud workshops

- OAGi-NIST workshop on Open Cloud Architectures for Smart Manufacturing: identified and prioritized technology and standards' gaps for cloud-enabled manufacturing services

- NIST Workshop on Cloud-Based Applications for Sustainable Manufacturing: defined a standards' strategy to use data from process measurements to quantify manufacturing process sustainability.

\section{A \\ INTERNATIONAL}

Standards Worldwide

\section{OAGi \\ Open Applications Group}

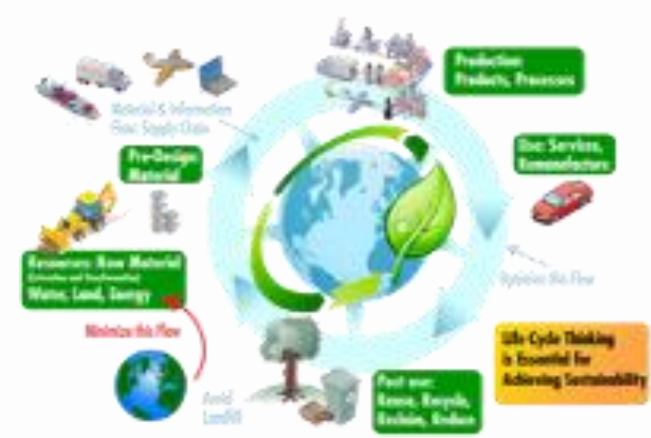




\section{Smart Manufacturing Operations Planning and Control}

- How can you use sensors, data, and computation to assess machine health, optimize maintenance, and avoid downtime?

- How can you use wireless communications in industrial environments for more flexible manufacturing?

- How do you secure the computers and networks that control manufacturing operations?

- How can you use the same digital model to support the entire product lifecycle, from design to production to service and sustainment?

- How can you integrate different analysis tools to improve manufacturing operations?

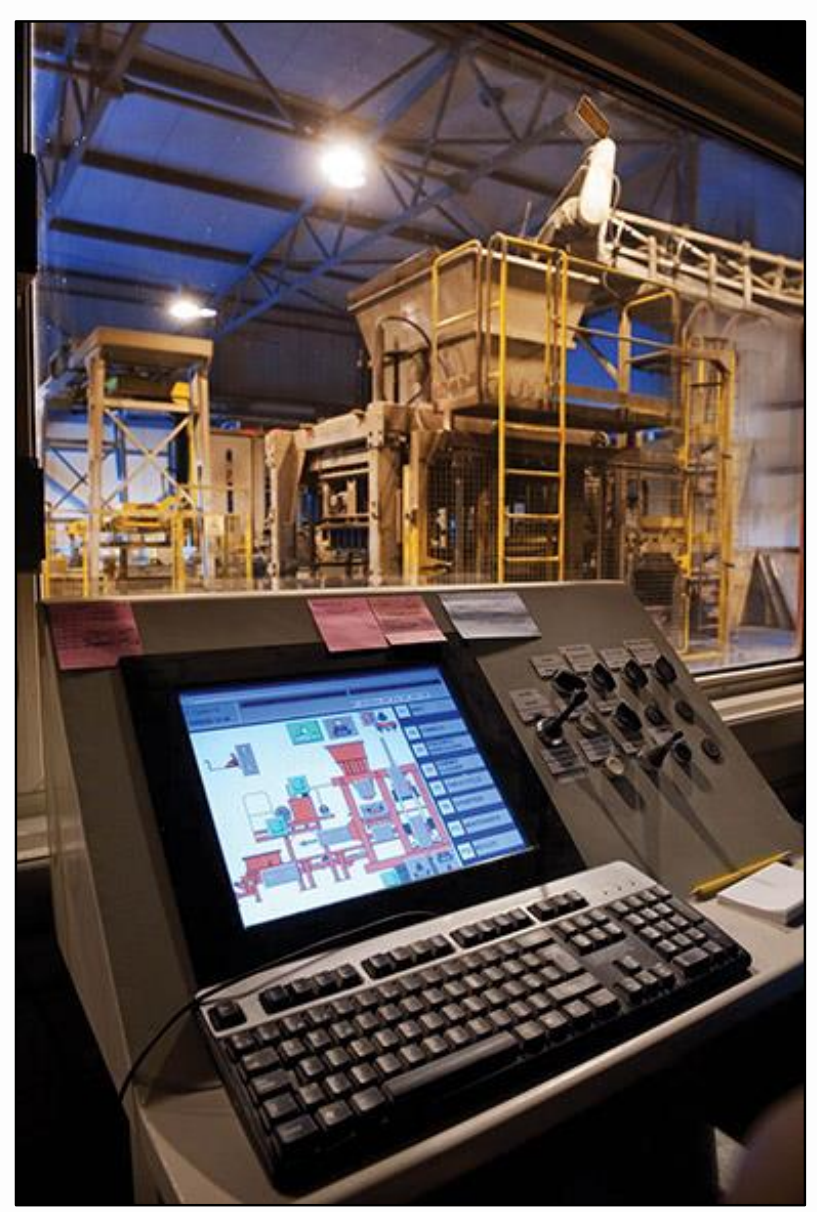




\section{Standards Contributions}

- STEP AP 242 (ISO 10303-242) standard on Managed Model Based 3D Engineering

- Provides for interoperability of Product Lifecycle Management (PLM) information to enable the "digital thread" of model-based information for manufacturing, to reduce costs and improve responsiveness.

- NIST Special Publication 800-82 Guide to Industrial Control Systems Security

- Provides guidance on how to secure industrial control systems while addressing their unique performance, reliability, and safety requirements

- Quality Information Framework (QIF) standard

- Streamlines the flow of quality information across the complete product-quality lifecycle.

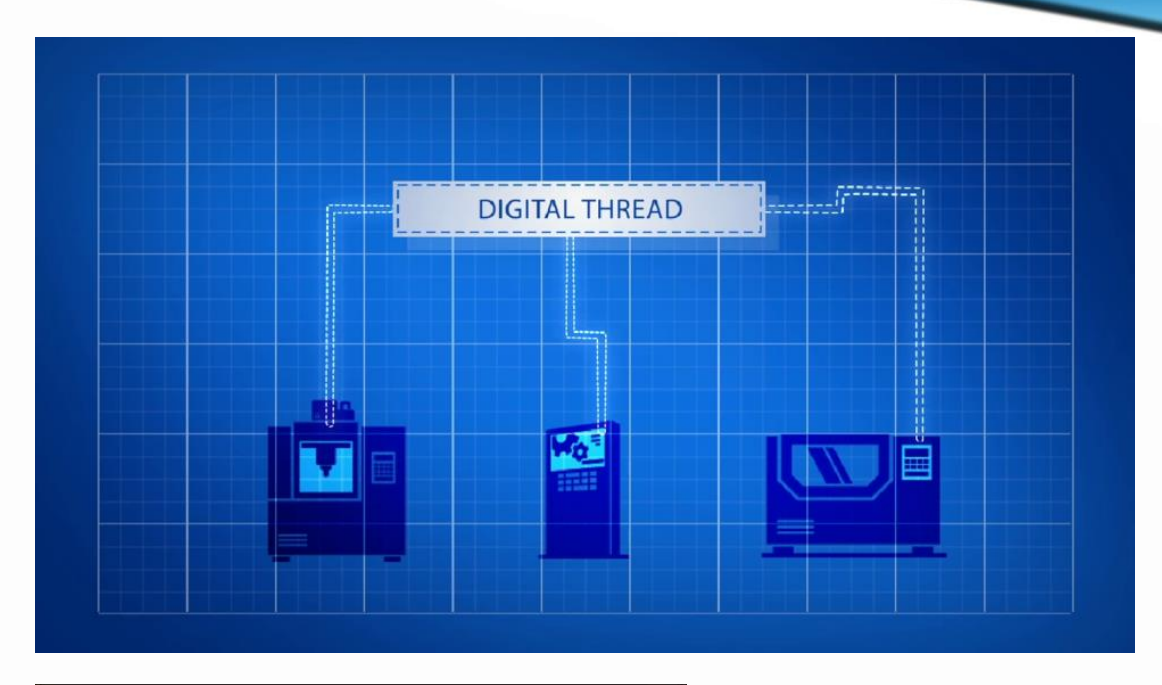

NIST Special Publication $800-82$

Guide to Industrial Control Systems (ICS) Security 


\section{Hot Off the Press: NIST Guide to Industrial}

\section{Wireless Systems Deployments}

- Industrial Wireless Fundamentals

- Business Case for Wireless

- Wireless Lifecycle

- Wireless for Safety

- Industrial Wireless Security

- Best Practice Considerations

- Checklists

- Wireless Applicability Matrix

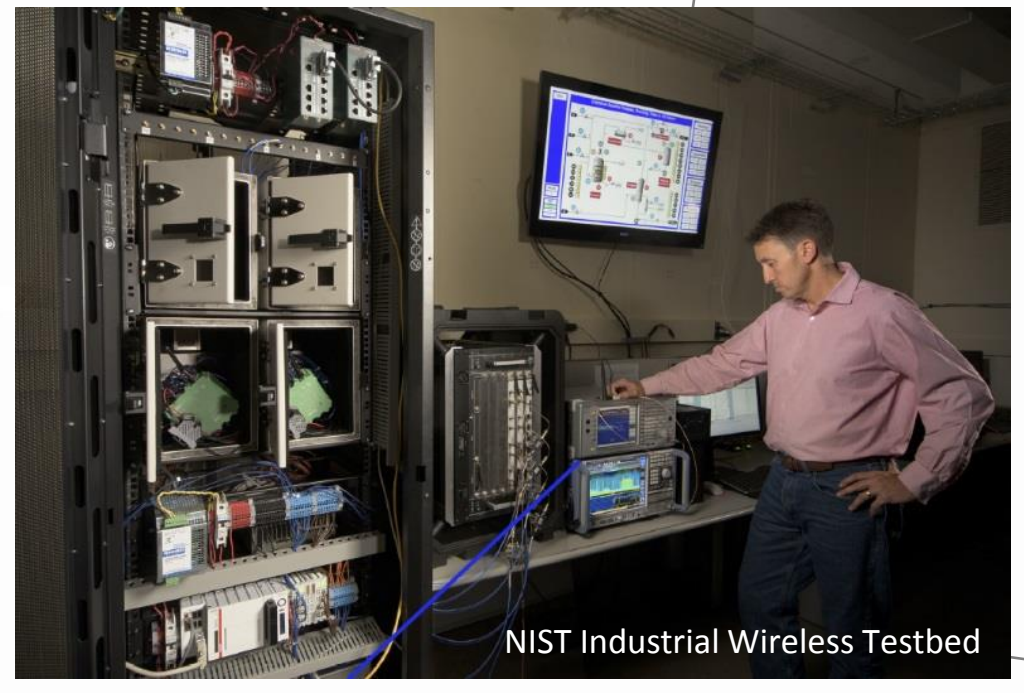

NIST Advanced Manufacturing Series 300-4

Guide to Industrial Wireless

Systems Deployments
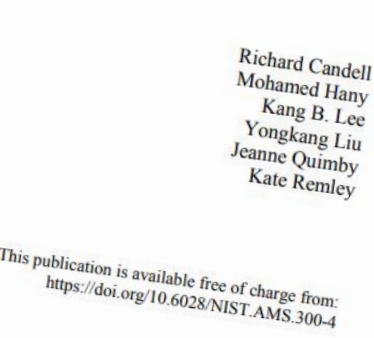

NLST 


\section{Prognostics, Health Management, and Control Project}

- Manufacturing Process and Equipment Monitoring

- Health and Control Management for Robot Systems

- Robot Positioning Performance Degradation

- Workcell-level PHM V\&V

- Machine Tool Linear Axes Diagnostics and Prognostics
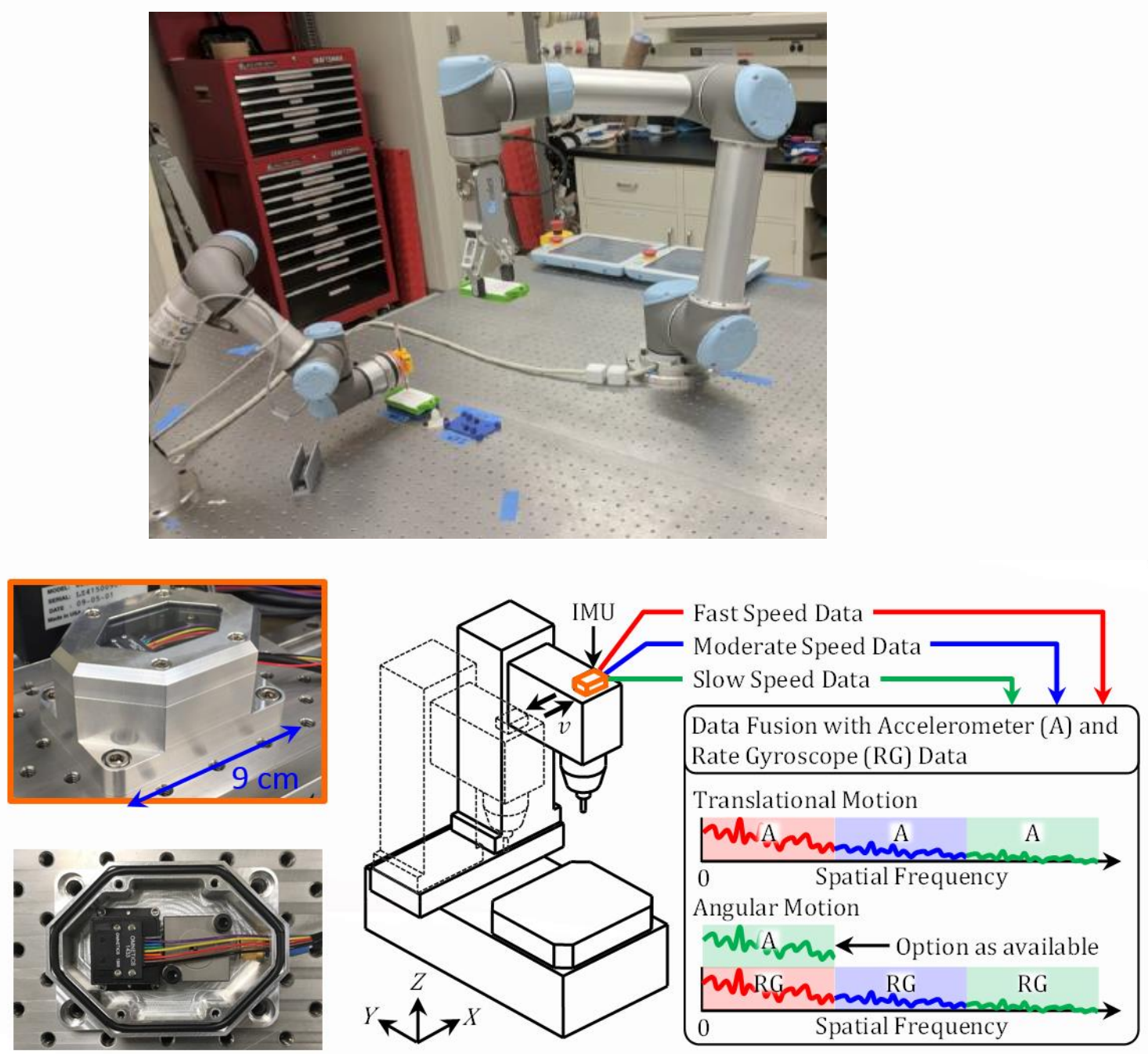

IMU for Linear Axis Monitoring 


\section{ASME Standards Meeting - Monitoring, Diagnostics, and Prognostics for Manufacturing Operations}

\section{Here, Friday! Discussion of:}

- Standardized Terminology for PHM Guideline on Data and Collection Strategies

- Guideline to Determine What Health Data to Capture and Collection Strategies to Employ

- Guideline to Determine What Sensors and Where They Should Be Employed to Inform on Process/Equipment Health

- Guideline for Implementing Sensor Data Fusion/Multi-modal Data Fusion

- Guideline to Determine When and Where PHM Should Be Added/Integrated

- Expand MTConnect/Data Communications 


\section{Working With Others}

\section{Major stakeholder groups}

- Manufacturing enterprises

- Software vendors \& equipment providers

- Small and medium sized enterprises (SMEs)

- Industry consortia and standards developing organizations

- Government agencies

- Universities and research organizations

Modes of engagement

- Consortia, standards developing organizations

- Workshops, conferences, summits

- Site visits

- Cooperative Agreements

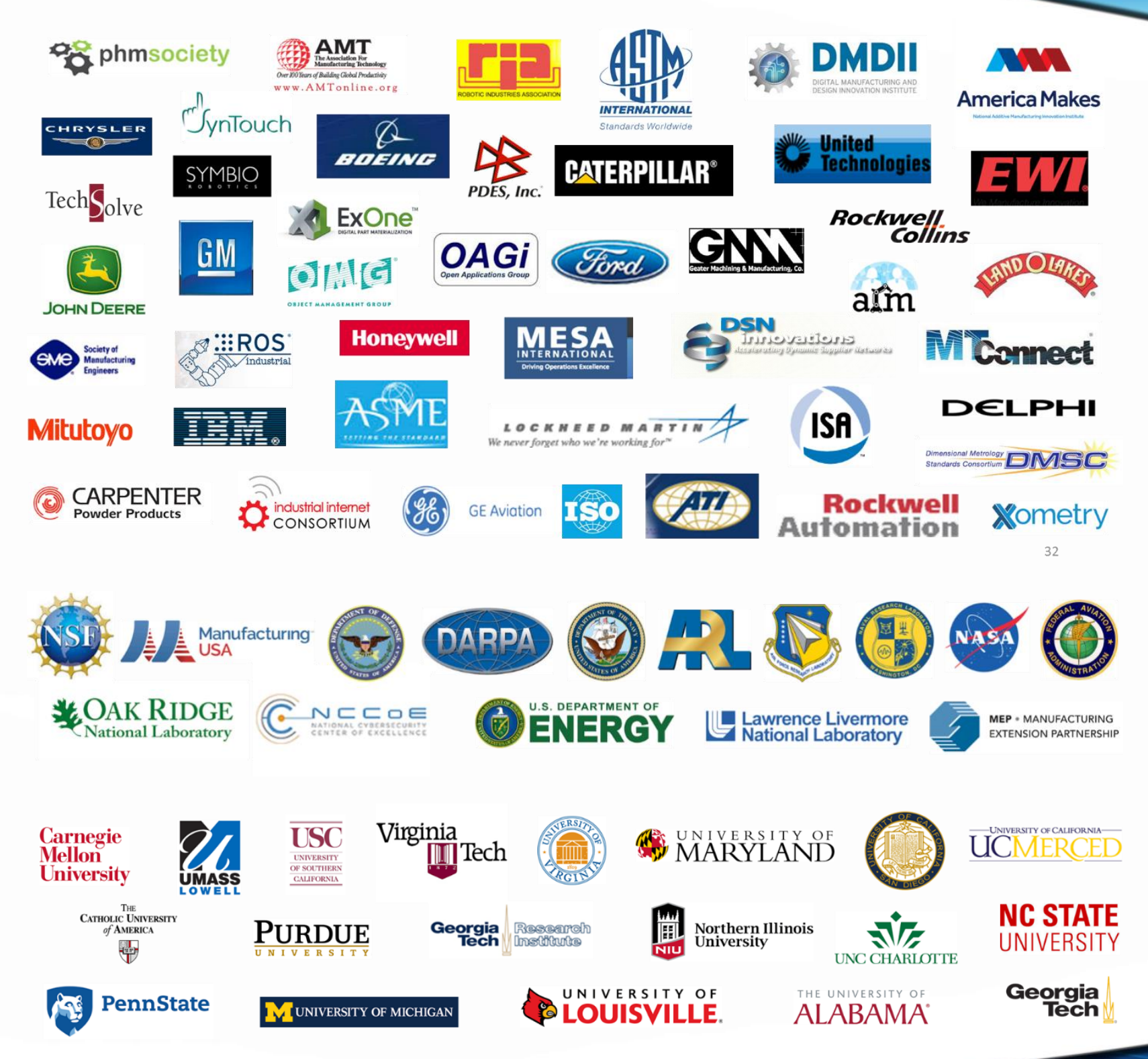




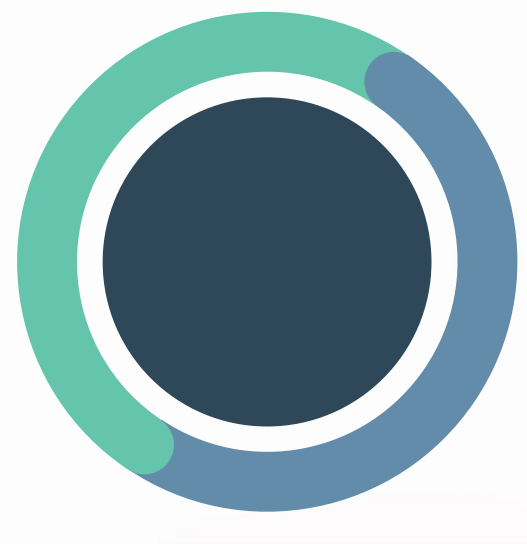

What measurement science and standards are needed to drive innovation and reduce risks of adoption of emerging/disruptive PHM technologies? 


\section{Contact Information}

Thank you!

Albert Wavering

Chief, Intelligent Systems Division

3019753418

Questions?

albert.wavering@nist.gov

NIST Engineering Laboratory 100 Bureau Drive, Mail Stop 8260

Gaithersburg, MD 20899-8260

www.nist.gov/el 


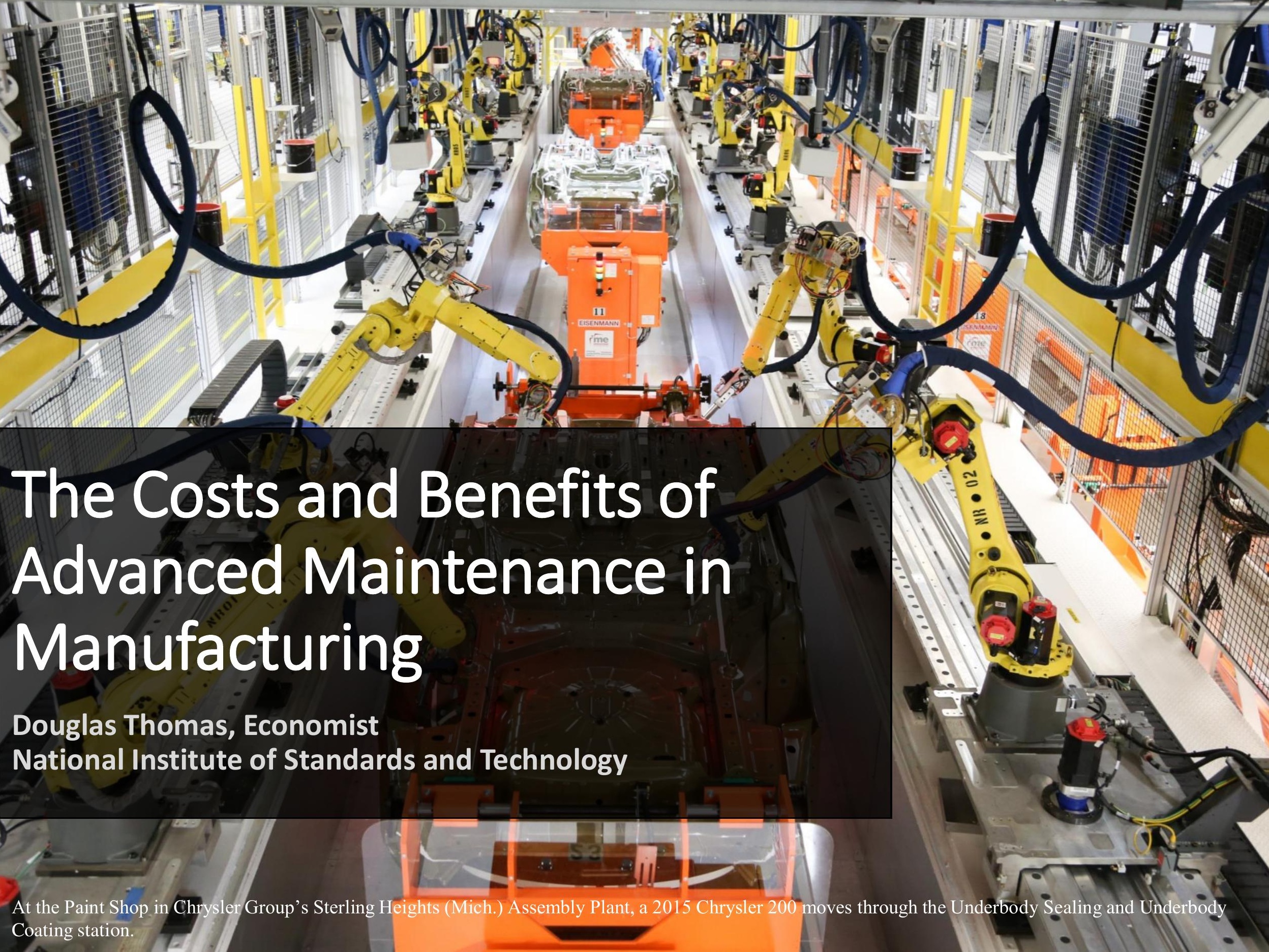




\section{Overview}

Estimating national costs/benefits associated with adopting advanced maintenance

- Current literature/data

- Maintenance costs

- Benefits of predictive maintenance

- Barriers to adoption

- Current maintenance practice

- Data needs

- Feasibility of collecting data

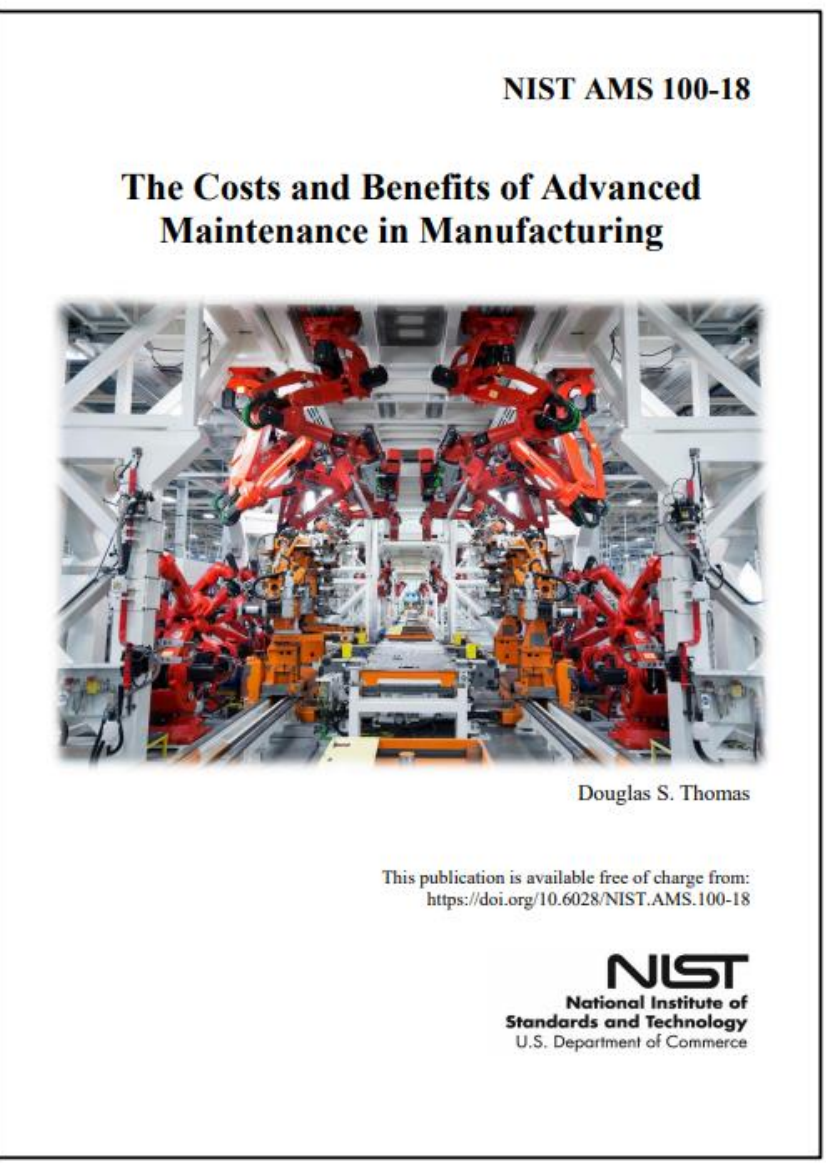




\section{Maintenance Cost: Data}

- Economic Census

- Maintenance outsourcing

- Includes machinery and buildings

- Bureau of Economic Analysis

- Maintenance outsourcing

- Machinery only

- Bureau of Labor Statistics

- Labor only

- Excludes overhead/materials

- Estimates of cost require making some assumptions 


\section{Maintenance Cost: Literature}

- Varying terminology

- Reactive, Preventive, Predictive

- Cost studies

- Varying countries (e.g., Sweden, Belgium)

- Varying economic metrics

- Case studies with

- Varying types of machinery

- Manufacturing and nonmanufacturing
Characteristics of Maintenance Costs from a Selection of Articles, Various

Countries/Industries

\begin{tabular}{lcc}
\hline & \multicolumn{2}{c}{ Maintenance } \\
\hline Description & Low & High \\
Cost of Goods Solda,b $^{\mathrm{a}}$ & $15.0 \%$ & $70.0 \%$ \\
Sales $^{\mathrm{c}}$ & $0.5 \%$ & $25.0 \%$ \\
Cost of Ownership $^{\mathrm{d}}$ & \multicolumn{2}{c}{$37.5 \%$} \\
$\begin{array}{l}\text { Replacement Value of Plant } \\
\text { Cost of Manufacturing }\end{array}$ & $1.8 \% \quad 5.0 \%$ \\
$\begin{array}{l}\text { Percent of Planned } \\
\text { Production Time that is }\end{array}$ & $23.9 \%$ \\
Downtime & \\
\hline
\end{tabular}




\section{Benefits of Adoption}

- Similar challenges

- Varying countries

- Varying metrics

- Varying industries

- Varying terminology

- Case studies

- Limits to extrapolating

- Wide range of impacts
Range of Impacts Identified in Various Publications for Implementing Advanced Maintenance Techniques

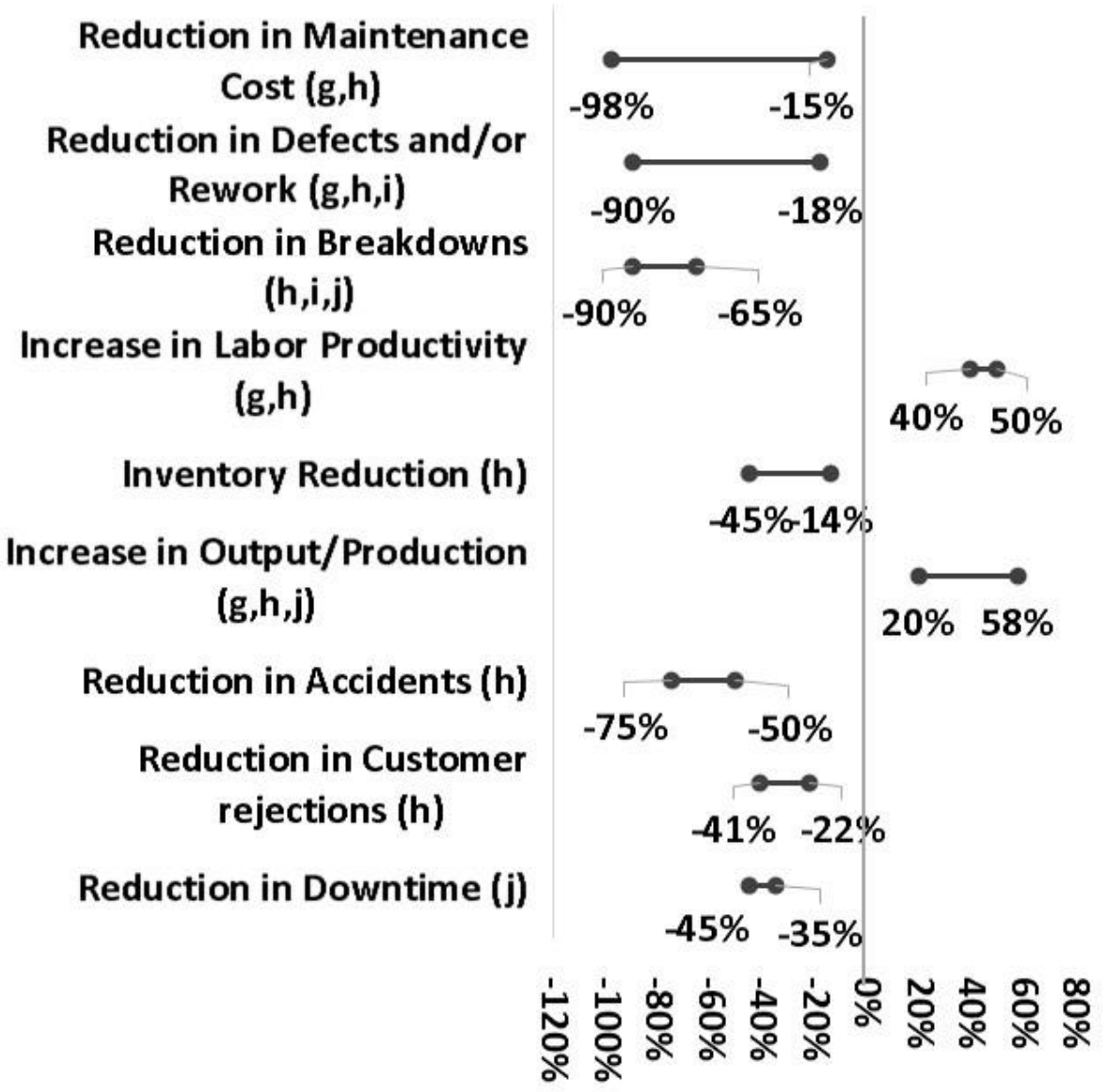




\section{Maintenance Cost Characterization,}

\section{by Type}

\begin{tabular}{|c|c|c|c|}
\hline & & Maintenance Type & \\
\hline & Reactive & Preventive & Predictive \\
\hline Frequency & On Demand & Scheduled, Timed, or Cycle Based & Condition Based \\
\hline Labor Cost & High & High & Low \\
\hline Labor Utilization & High & Low & Low \\
\hline Parts Cost & High & Medium & Medium \\
\hline $\begin{array}{l}\text { Throughput } \\
\text { Impact }\end{array}$ & High & Medium & Very Low \\
\hline Urgency & High & Low & Low \\
\hline ROI & Low & Medium & High \\
\hline $\begin{array}{l}\text { Initial } \\
\text { Investment }\end{array}$ & Low & Medium & High \\
\hline Profitability & Not cost effective & Satisfactory cost-effectiveness & $\begin{array}{l}\text { Significant cost } \\
\text { savings }\end{array}$ \\
\hline $\begin{array}{l}\text { Cost } \\
\text { effectiveness }\end{array}$ & Labor intensive & $\begin{array}{l}\text { Costly due to potential over } \\
\text { maintenance or ineffective \& } \\
\text { inefficient maintenance }\end{array}$ & $\begin{array}{l}\text { Cost-effective due to } \\
\text { extended life and less } \\
\text { failure-induced costs }\end{array}$ \\
\hline
\end{tabular}




\section{Current Maintenance Practice}

- Studies have varying factors (e.g., country)

- Firm competition

- Cost comp. - higher reactive

- Quality comp. - higher predictive

- Swedish study - 50\% of maintenance time is planned tasks

- $13 \%$ planning

- $37 \%$ unplanned 


\section{Objectives and Prevalent Barriers to the Adoption of Advanced Maintenance Techniques}

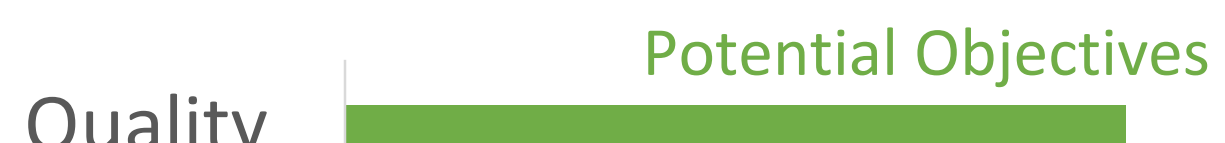

Productivity

Availability and Reliability

Safety and Environment

Organizational Readiness

\section{Potential Barriers}

Human Resource

Technology Support

Cost

$0 \%$

$50 \%$

$100 \%$ 


\section{Assessing the costs and benefits}

- To assess costs/benefits at National level

- Identify data needs

- Develop a data collection strategy

- Develop a scaling strategy

- Assess the minimum sample size 


\section{Data Needs Map}

Data needed

Some data availability

$\square$ Descriptive Grouping

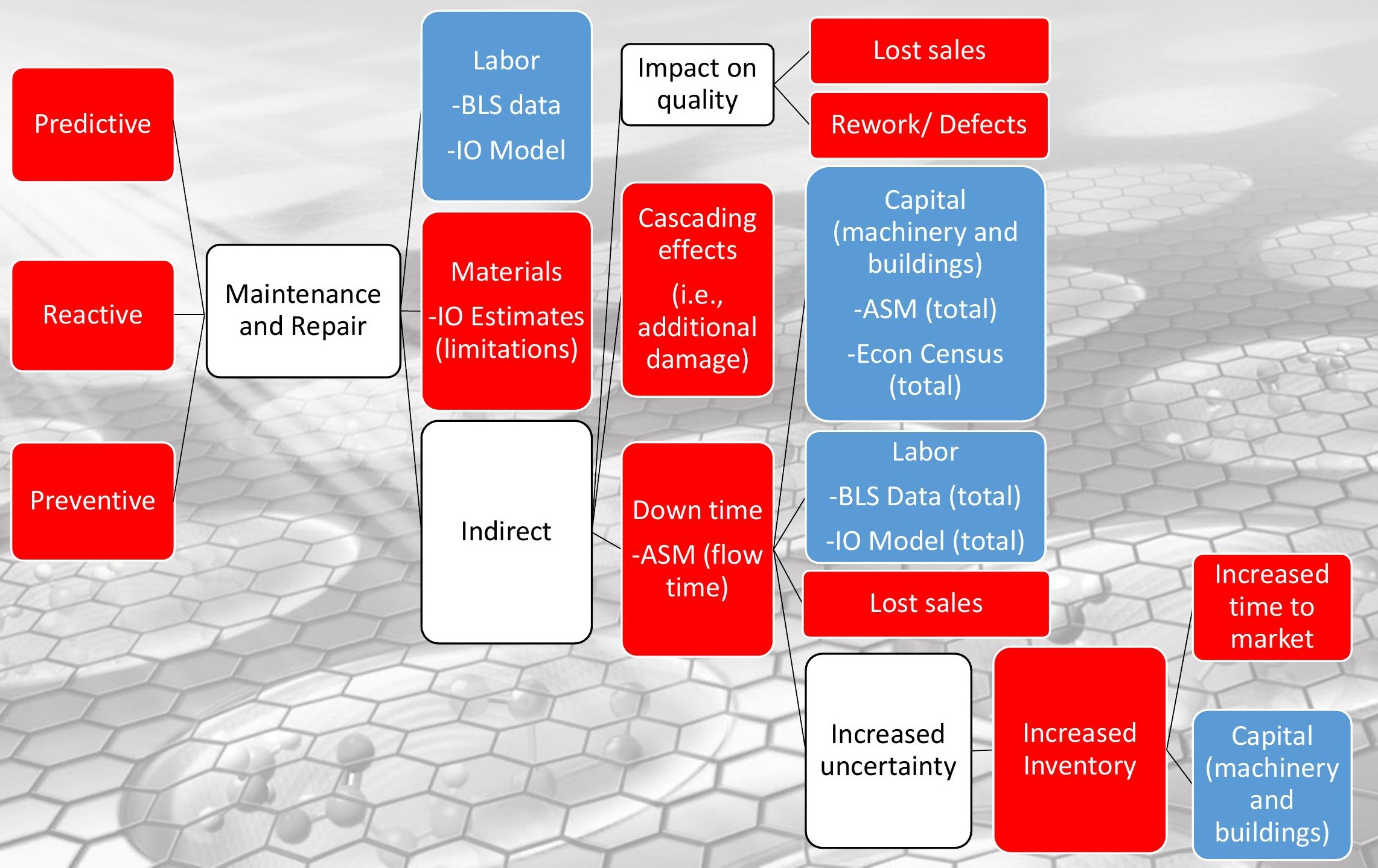




\section{Data Collection via}

Survey

- Collect data through survey

- Direct maintenance costs

- Downtime

- Defects/rework

- Separate costs into predictive, preventive, and reactive

- Separate planned maintenance from repair

- Lost sales $\rightarrow$ quality

- Scale using payroll data by industry by establishment size

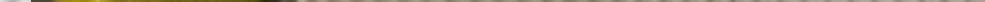




\section{Data Collection via}

Survey

- Disproportional amount of small firms

- Scale by establishment size

- Census data

- Anonymous survey

- Short survey

- Target: 1 Page

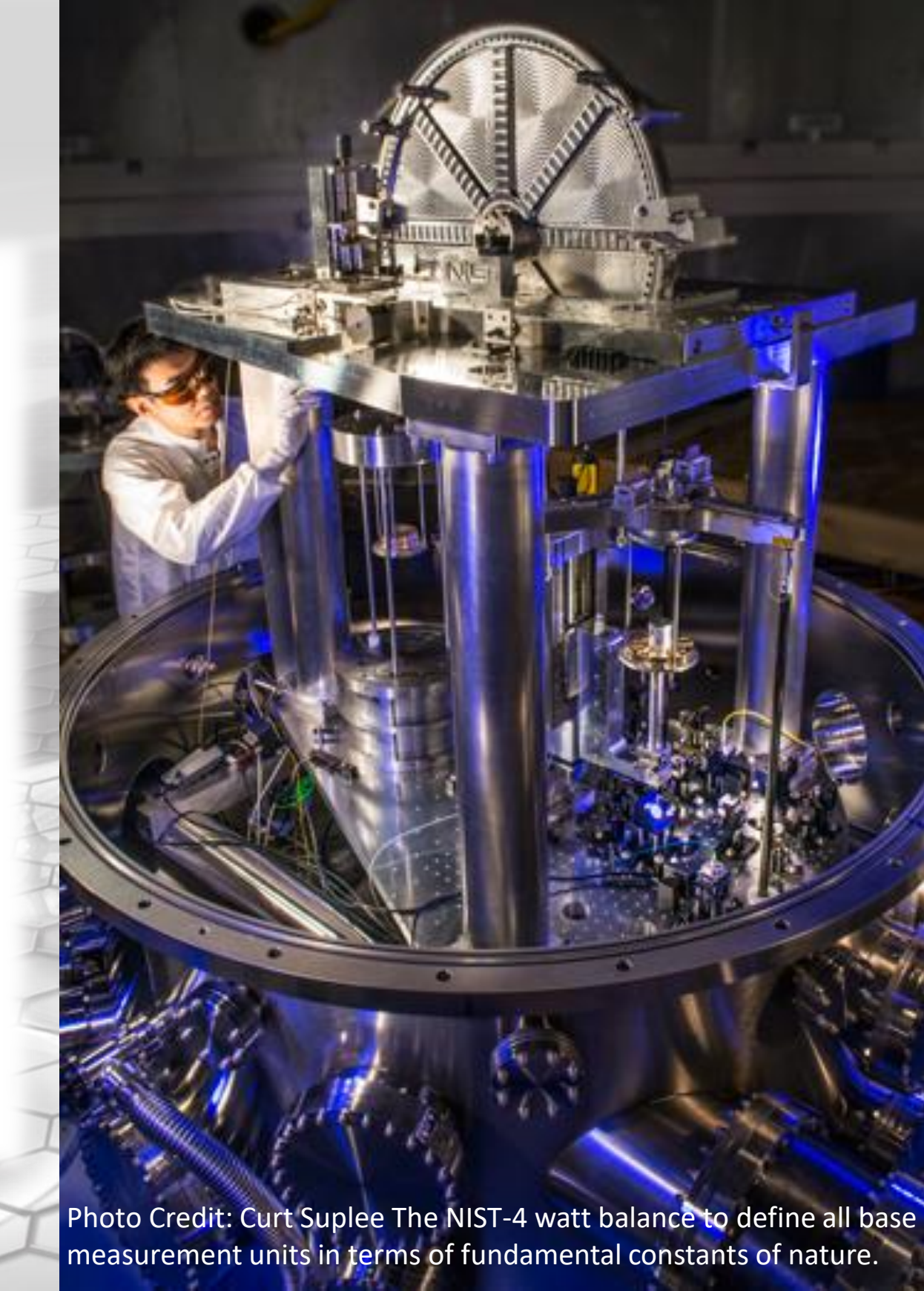




\section{Feasibility of Data Collection}

- Discussions with manufacturers suggest

- It is reasonable to expect manufacturers to be willing and able to share data

- However,

- Apprehensiveness from a few in sharing some of the variables

- A number of variables are not tracked $\rightarrow$ approximations 


\section{Required Sample Size for Survey}

It's complicated

$$
\text { Sample Size }=\left(\frac{z \sigma}{e}\right)^{2}
$$

where

$\sigma=$ Standard deviation

$e=$ Margin of error

$z=\mathrm{z}$-score

Estimate standard deviation using census data on maintenance cost

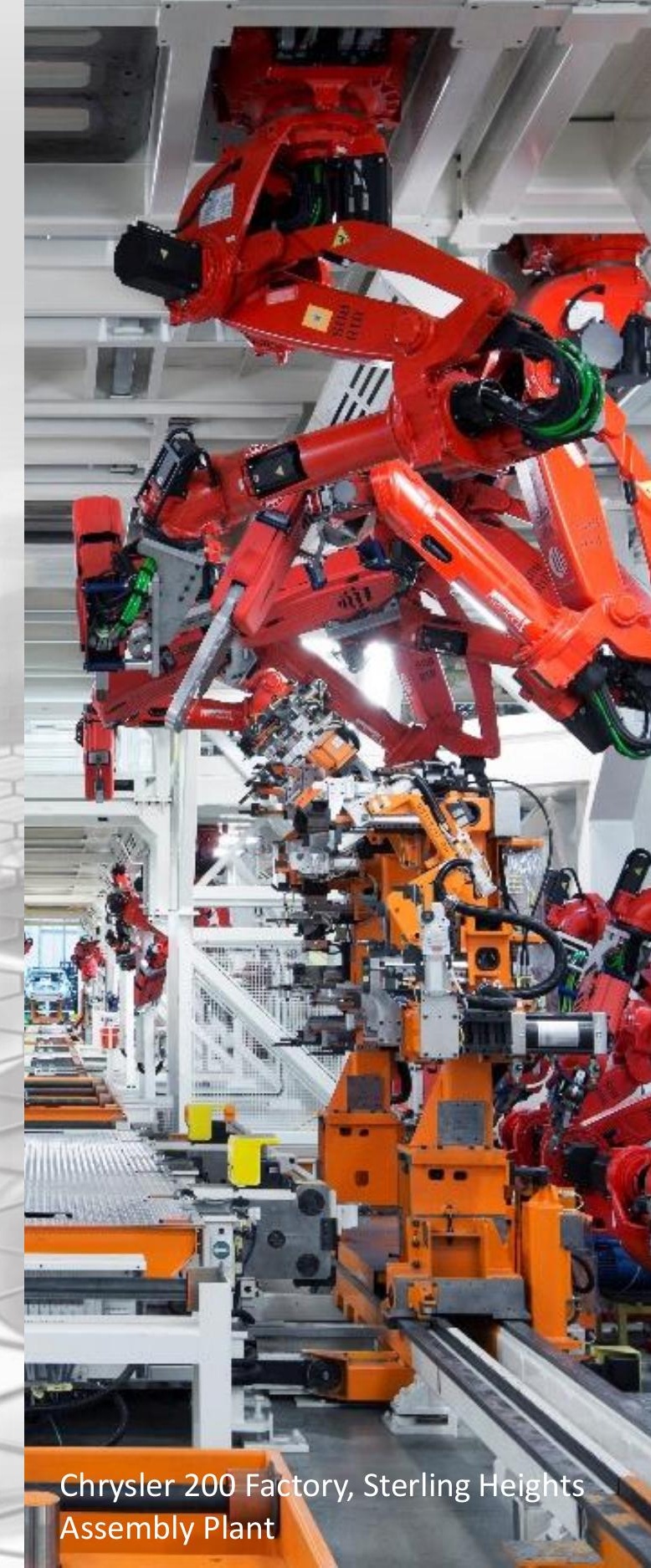




\section{Sample Size to Estimate Maint. Cost}

- Graph sample size

- Standard deviation from Census

- Different confidence intervals

- $10 \%$ margin of error w/95\% confidence interval: 77

- $20 \%$ margin of error w/90\% confidence interval: 14

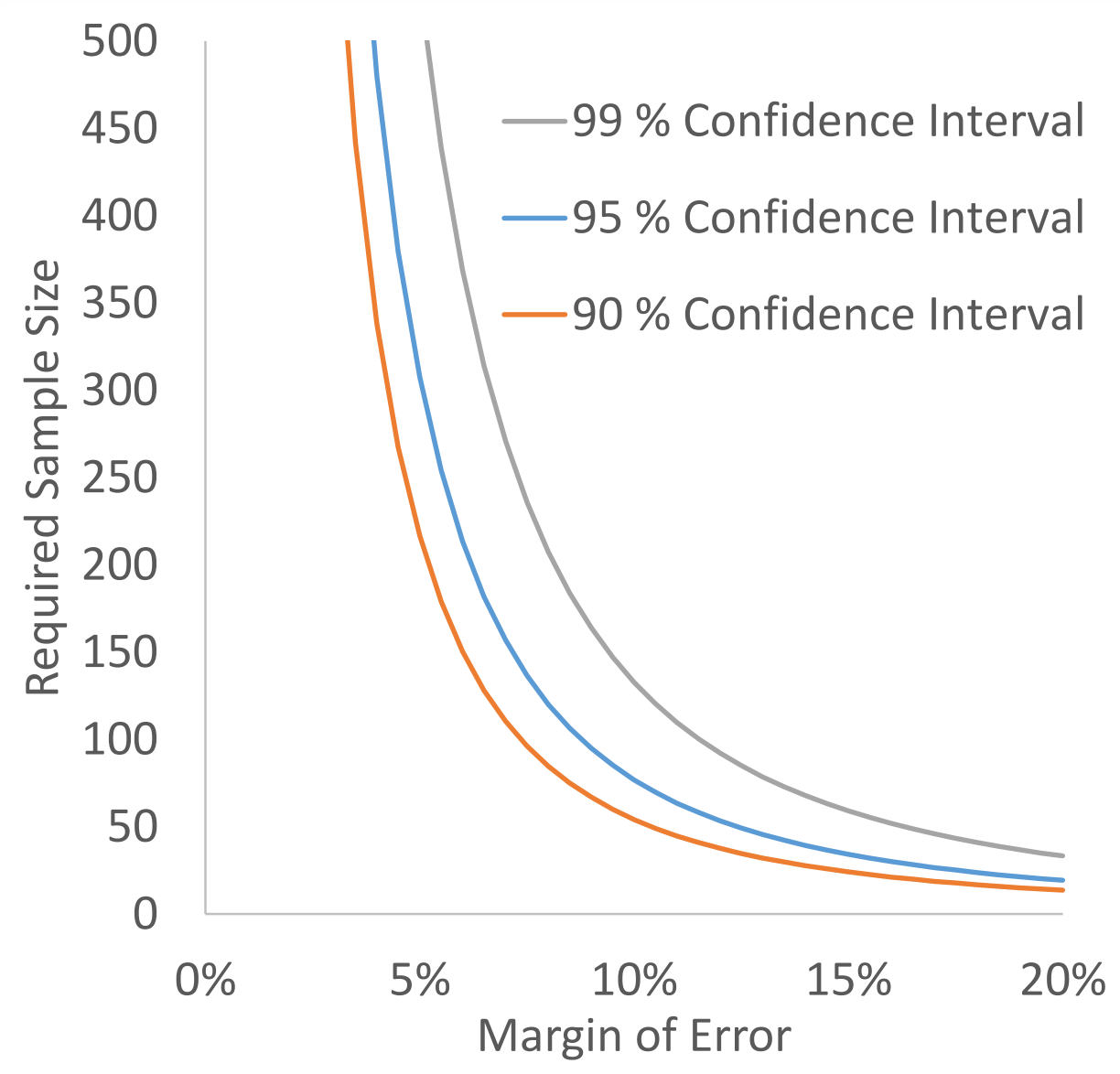




\section{Summary}

- Current maintenance cost data has limitations

- Outsourcing only

- Includes buildings + machinery

- Literature has

- Varying metrics

- Varying countries

- Wide range of values

- Feasibility of data collection

- Firms are willing/able

- Approximations

- Minimum sample size: 14-77 needed

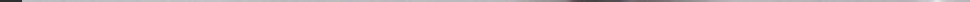




\section{How You Can Help}

- Your participation would be appreciated

- What's in it for you?

- Receive a copy of the report

- See how you compare with others

- Develop the business case for advanced maintenance

How to participate in Survey

Contact Douglas Thomas

douglas.thomas@nist.gov

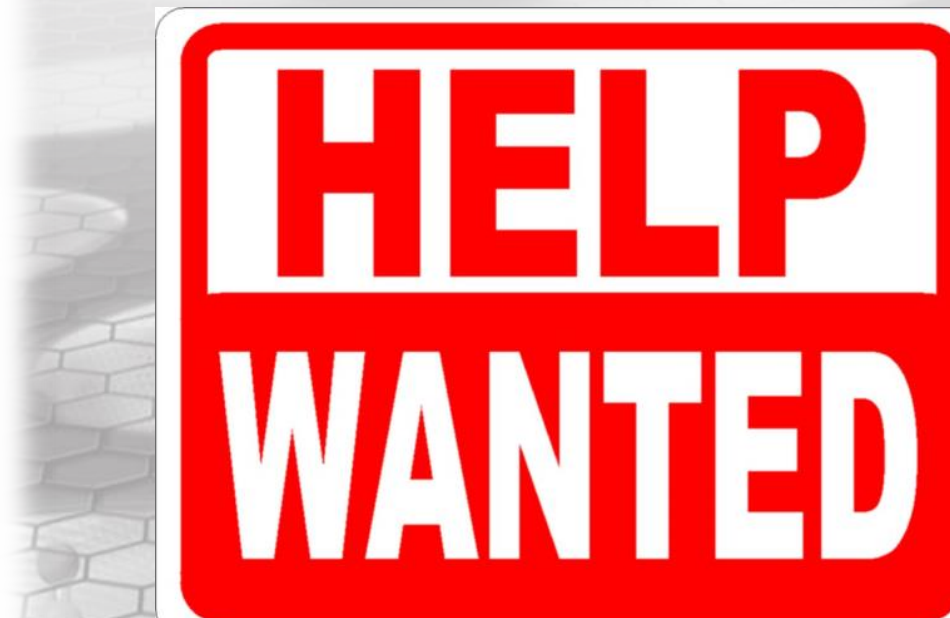

This Photo by Unknown Author is licensed under CC BY-NC-

\section{Thank You}




\section{Sources}

- $\quad$ aMobley, R. Keith. An Introduction to Predictive Maintenance. (Woburn, MA: Elsevier Science, 2002). 1.

- ' bBevilacqua, M. and M. Braglia. "The Analytic Hierarchy Process Applied to Maintenance Strategy Selection." Reliability Engineering and System Safety. 70, no 1 (2000): 71-83.

- 'Komonen, Kari. "A Cost Model of Industrial Maintenance for Profitability Analysis and Benchmarking." International Journal of Production Economics. 79 (2002): 15-31.

- ' dHerrmann, C., S. Kara, S. Thiede. "Dynamic Life Cycle Costing Based on Lifetime Prediction." International Journal of Sustainable Engineering. 4, no 3 (2011): 224-235.

- eEti, M.C., S.O.T. Ogaji, and S.D. Probert. "Reducing the Cost of Preventive Maintenance (PM) through Adopting a Proactive Reliability-Focused Culture." Applied Energy. 83 (2006): 1235-1248.

- 'Tabikh, Mohamad. "Downtime Cost and Reduction Analysis: Survey Results." Master Thesis. KPP321. Mälardalen University. (2014). http://www.diva-portal.org/smash/get/diva2:757534/FULLTEXT01.pdf

- gNakajima, S. Introduction to Total Productive Maintenance (TPM). (Portland, OR: Productivity Press, 1988).

- " hAhuja, I.P.S. and J.S. Khamba. "Total Productive Maintenance: Literature Review and Directions." International Journal of Quality and Reliability Management. 25, no 7 (2008): 709-756.

- ' 'Chowdhury, C. "NITIE and HINDALCO give a new dimension to TPM." Udyog Pragati, Vol. 22 No. 1, (1995): 5-11.

- jFederal Energy Management Program. Operations and Maintenance Best Practices: A Guide to Achieving Operational Efficiency. (2010). https://energy.gov/sites/prod/files/2013/10/f3/omguide_complete.pdf 


\section{Other Sources}

- Barajas, Leandro and Narayan Srinivasa. "Real-Time Diagnostics, Prognostics and Health Management for Large-Scale Manufacturing Maintenance Systems" Proceedings of the 2008 International Manufacturing Science and Engineering Conference. October 7-10, 2008. Evanston IL.

- Jin, Xiaoning, David Siegel, Brian A. Weiss, Ellen Gamel, Wei Wang, and Ni Jun. "The Present Status and Future Growth of Maintenance in US Manufacturing: Results from a Pilot Survey." Manufacturing Review. 3 (2016): 1-10.

- Jin, Xiaoning, Brian A. Weiss, David Siegel, Jay Lee, Jun Ni. "Present Status and Future Growth of Advanced Maintenance Technology Strategy in US Manufacturing. 7, Issue 12 (2016): 1-18.

- Jin, Xiaoning, Brian Weiss, David Siegel, and Jay Lee. "Present Status and Future Growth of Advanced Maintenance Technology and Strategy in US Manufacturing." International Journal of Prognostics and Health Management. Special Issue on Smart Manufacturing PHM. 7, no 12 (2016).

- Jin, Xiaoning, David Siegel, Brian A. Weiss, Ellen Gamel, Wei Wang, and Ni Jun. "The Present Status and Future Growth of Maintenance in US Manufacturing: Results from a Pilot Survey." Manufacturing Review. 3 (2016): 1-10.

- Alsyouf, Imad. "Maintenance Practices in Swedish Industries: Survey Results." International Journal of Production Economics. 121 (2009): 212-223. 


\section{Emerging Sensing Technologies Towards Smart Machine Tools}

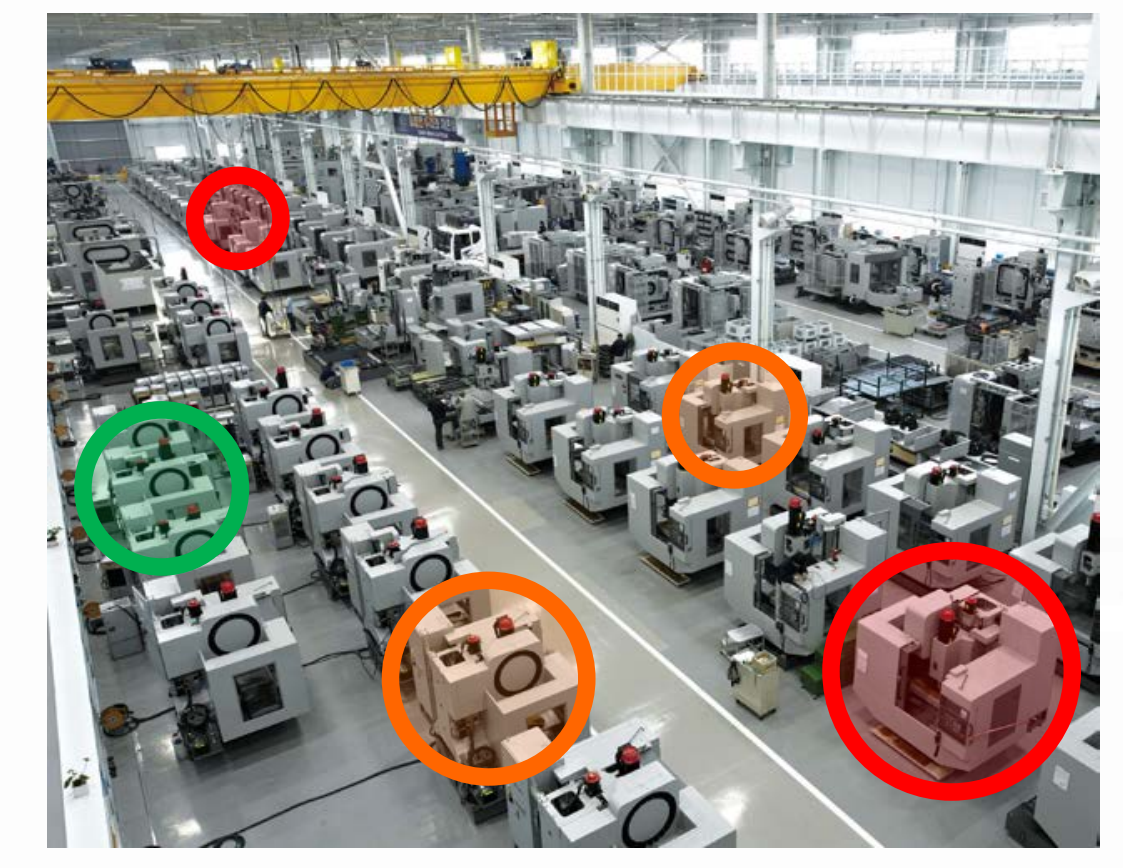

\section{Greg Vogl}

Intelligent Systems Division

Engineering Laboratory

National Institute of

Standards and Technology

(NIST)
Tablet

Machine \#1

Axis 1

$15 \mu \mathrm{m}$ range

Spalling detected

Spindle

Axis 2

$70 \mu \mathrm{rad}$ range

Optimum Machine: \#5

Official contribution of the National Institute of Standards and Technology (NIST); not subject to copyright in the United States. Certain commercial products, some of which are either registered or trademarked, are identified in this presentation in order to adequately specify certain procedures. In no case does such identification imply recommendation or endorsement by NIST, nor does it imply that the materials, equipment, or software identified are necessarily the best available for the purpose.

(1) 


\section{NIST $\rightarrow$ Economic Growth}

- NIST within U.S. Department of Commerce

- NIST promotes U.S. innovation by advancing measurement science, standards, and technology

- 6,500 Employees/Associates

- NIST partners with about 1,200 manufacturing specialists through manufacturing extensions
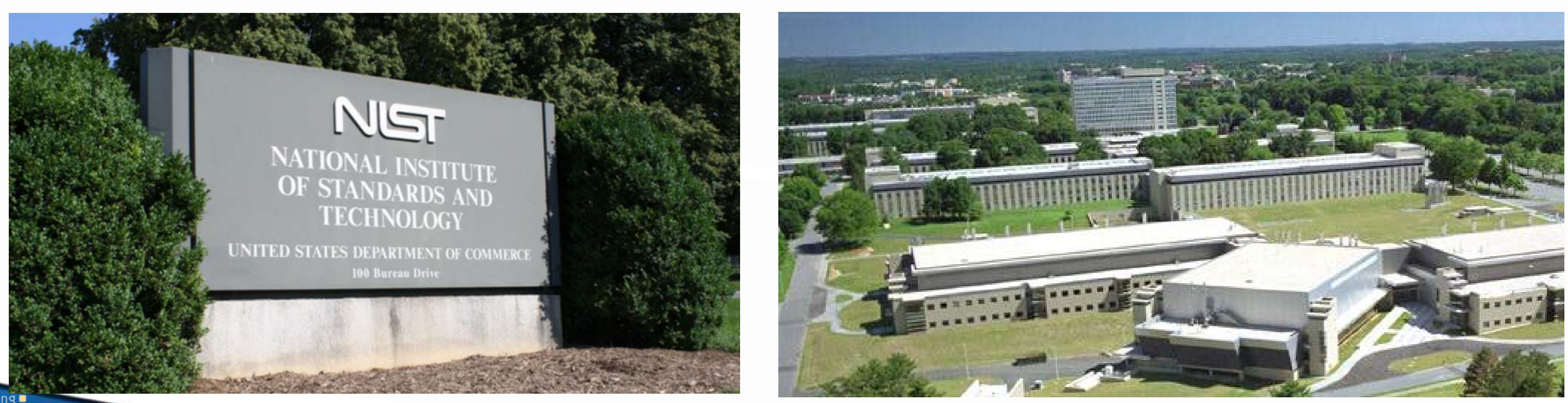

Prognostics,

Health Management, \& Control 


\section{Manufacturing = Economic Growth}

- Manufacturing = 12.5M U.S. jobs \& about $60 \%$ exports

- $2.8 \%$ economic growth for U.S. machine tool orders oxford Economics

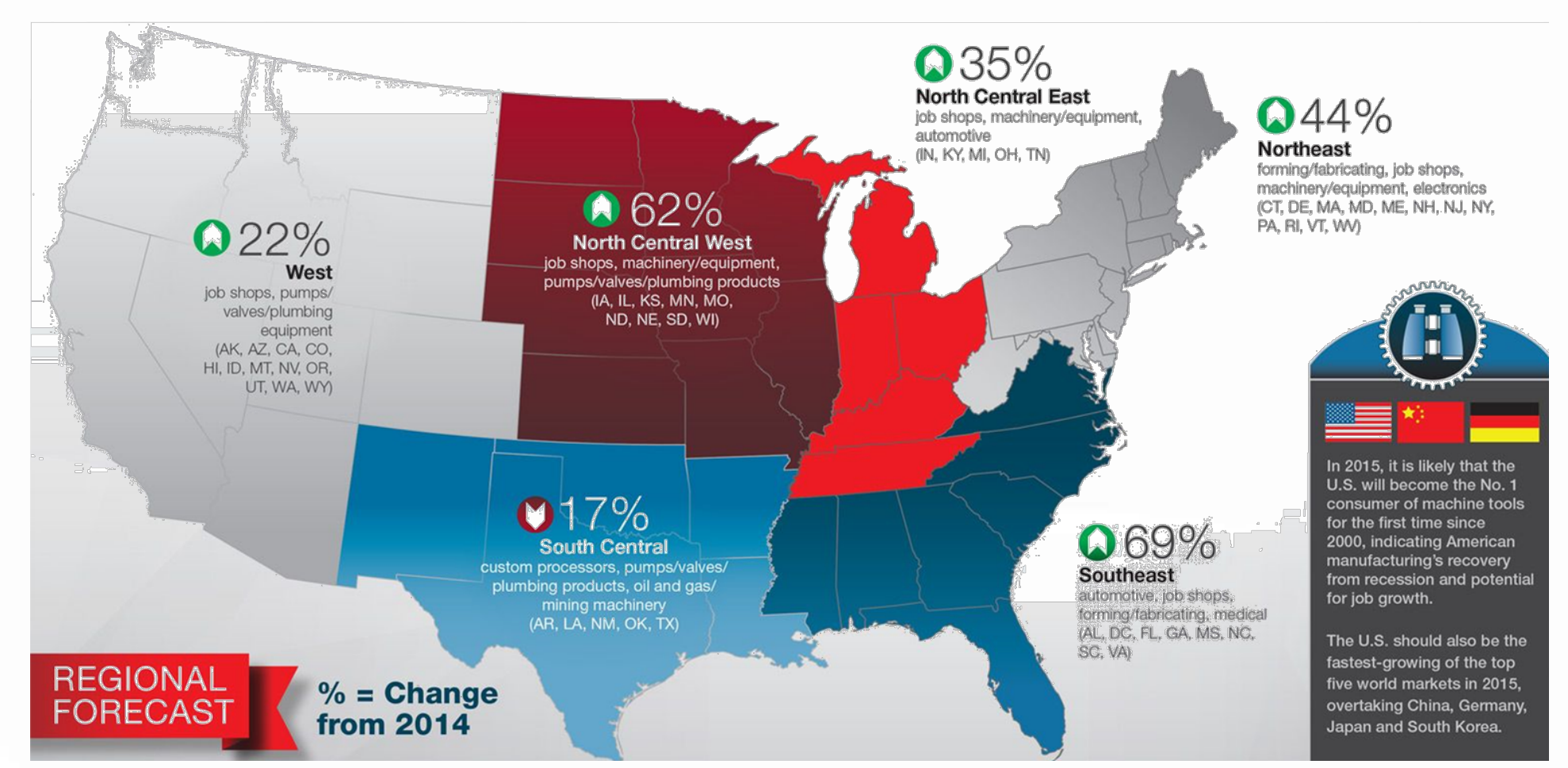

Modern Machine Shop, 2015 Capital Spending Survey \& Forecast

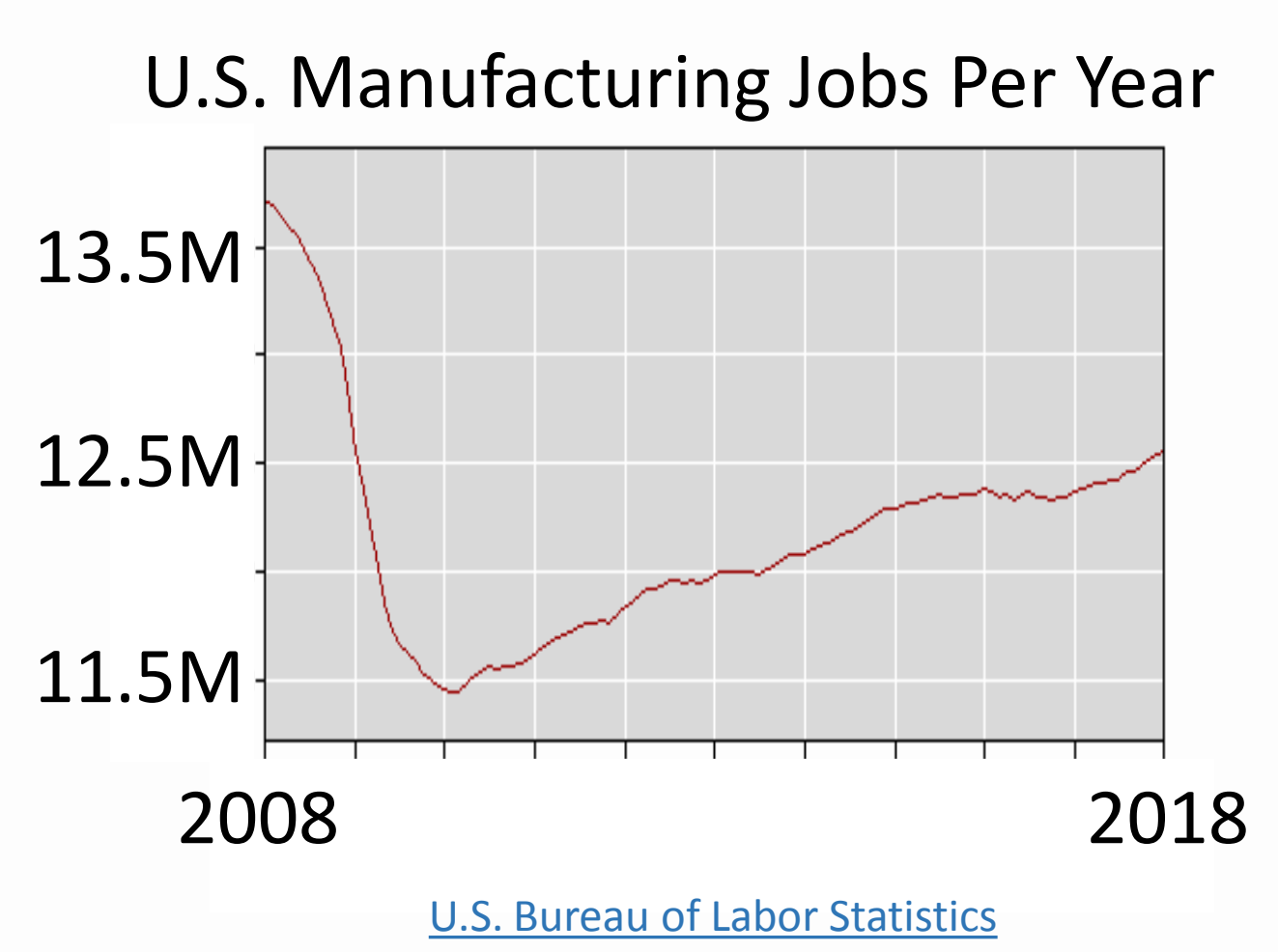


- 100 s of machine tools used in plants to mill precision parts

- 3+ axis motion

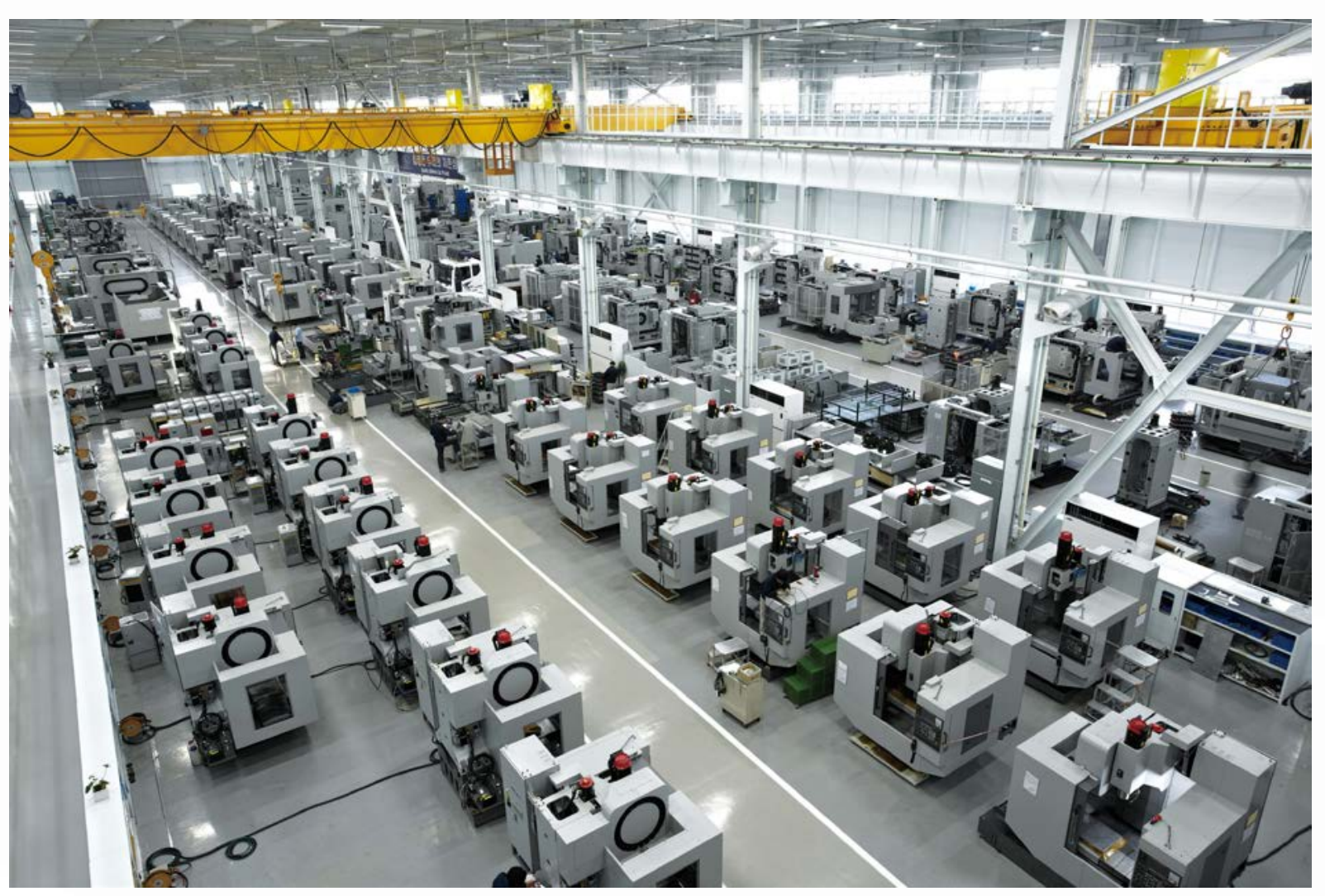

Hyundai Wia Plant

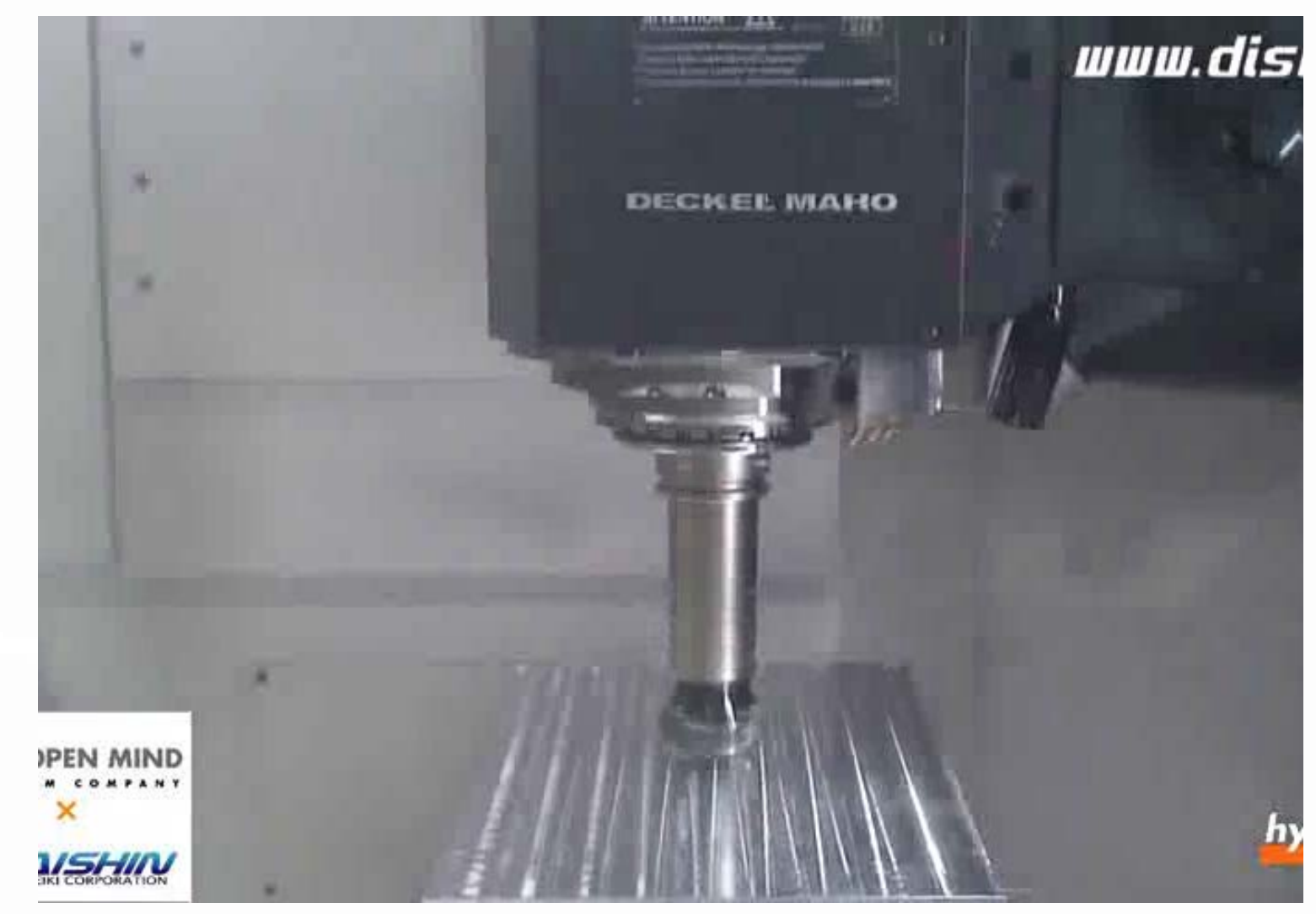

$\underline{5-A x i s ~ M a c h i n e ~ C u t t i n g ~ o f ~ H e l m e t ~(D a i s h i n ~ S e i k i ~ C o r p o r a t i o n) ~}$

1 (3) 


\section{Problem $=$ Unplanned Downtime}

- Faults/failures $\rightarrow 10$ s of \$Billions per year (> new machines!)

- Machine tool degradation causes performance changes and unplanned downtime

\section{Wear}

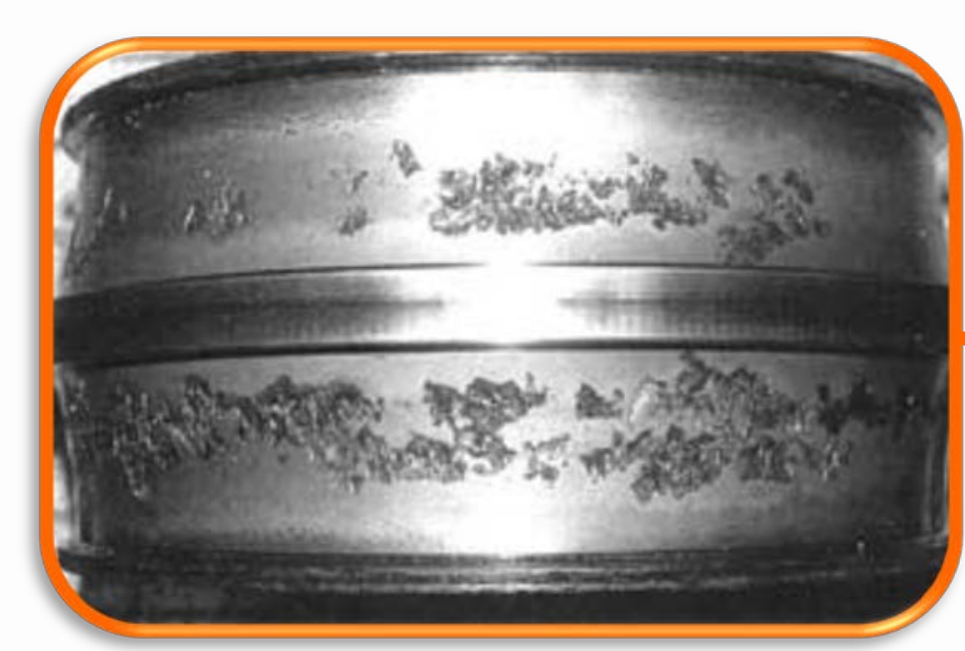

Machinery Lubrication (2004), Wear in Rolling Element Bearings and Gears

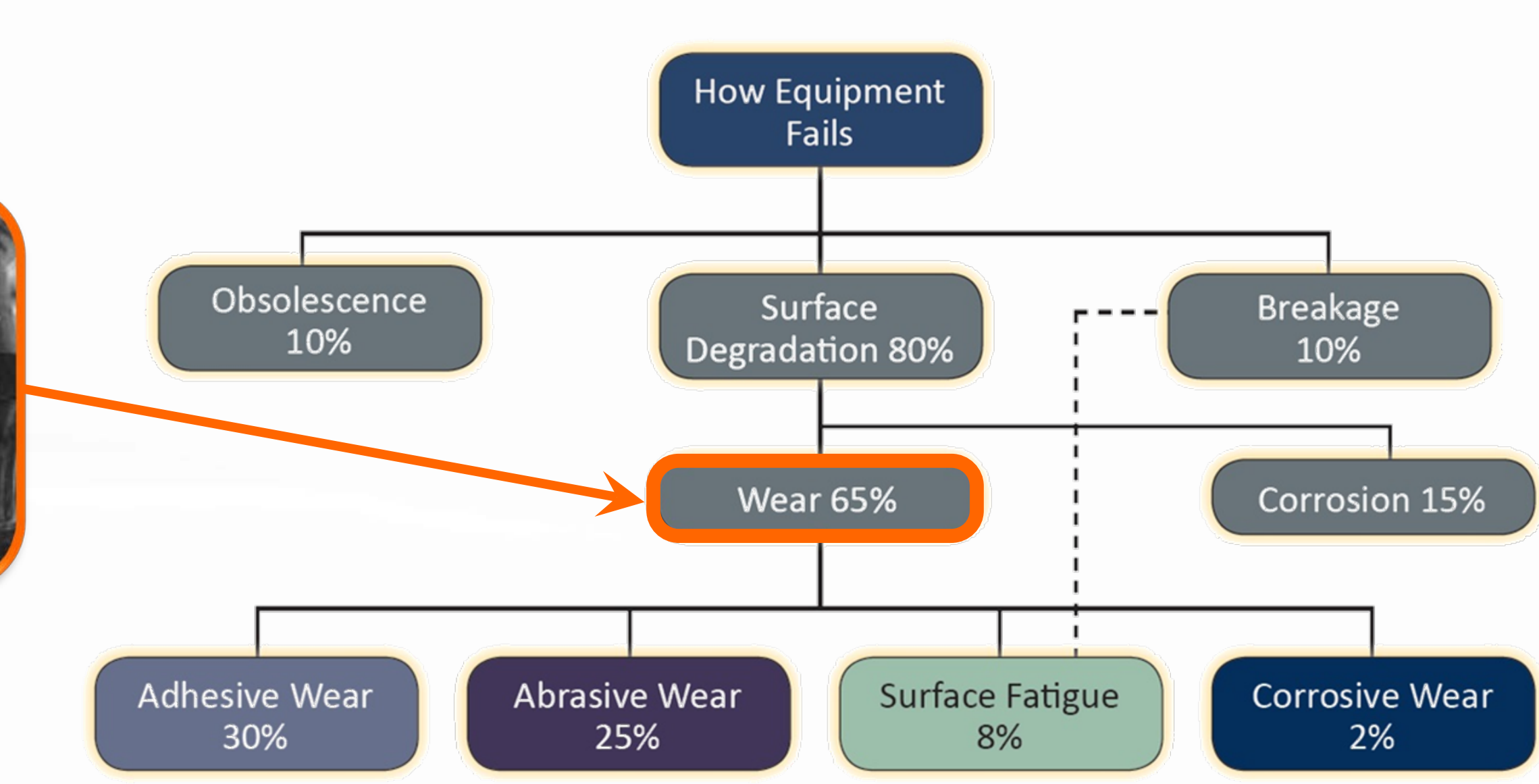




\section{Why Not Measure Health?}

- Major manufacturers say routine tracking of performance is too expensive

- Accuracy a pro, but setup and operation time/cost a con

- Offline

- Lack of periodic data

- Expensive
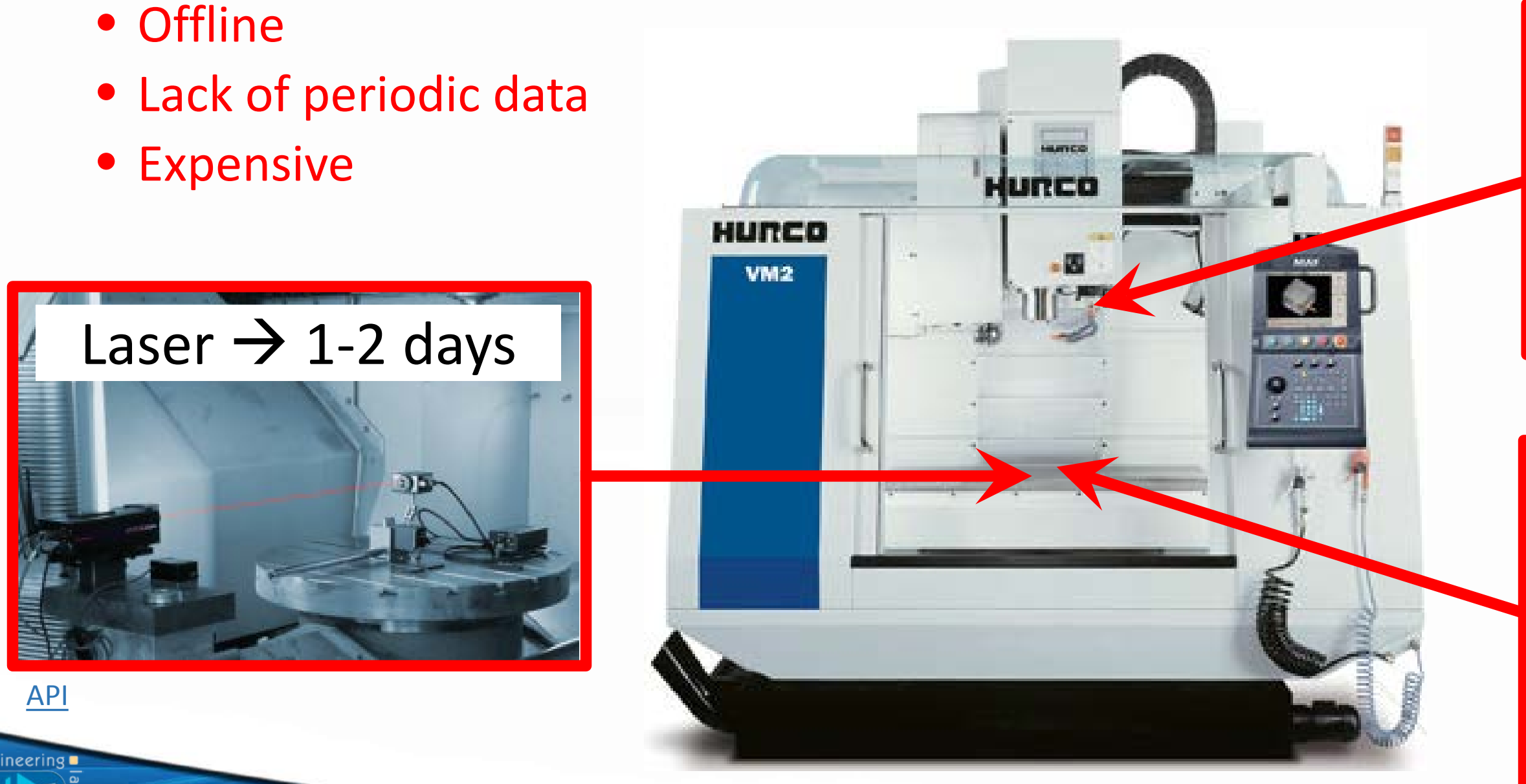

Cap probes $\rightarrow$ hours

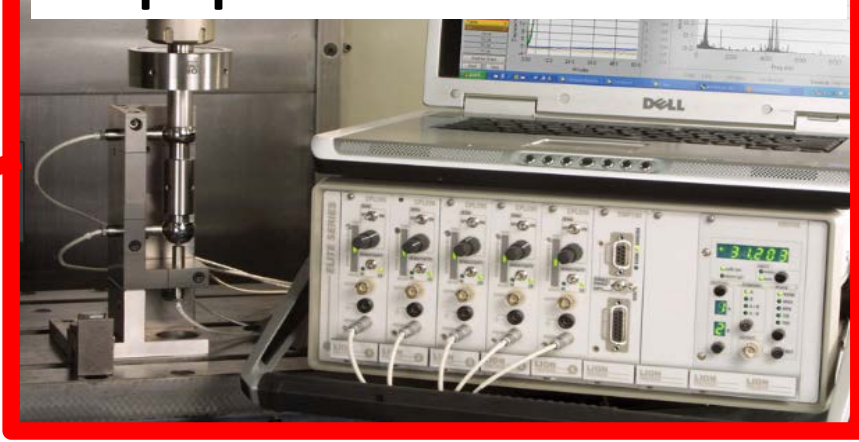

$\underline{\text { IBS Precision Engineering }}$

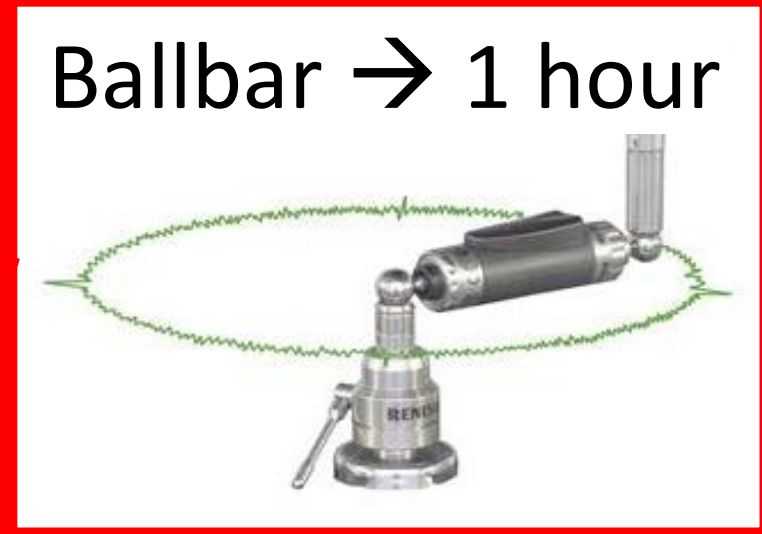




\section{GOAL: Smart Machine Tools}

- Industry challenge: "Machine health in 5 min?"

- On-machine measurement science to diagnose performance and root-causes

- Offline Online

- Lack of periodic data Data-rich

- Expensive Inexpensive

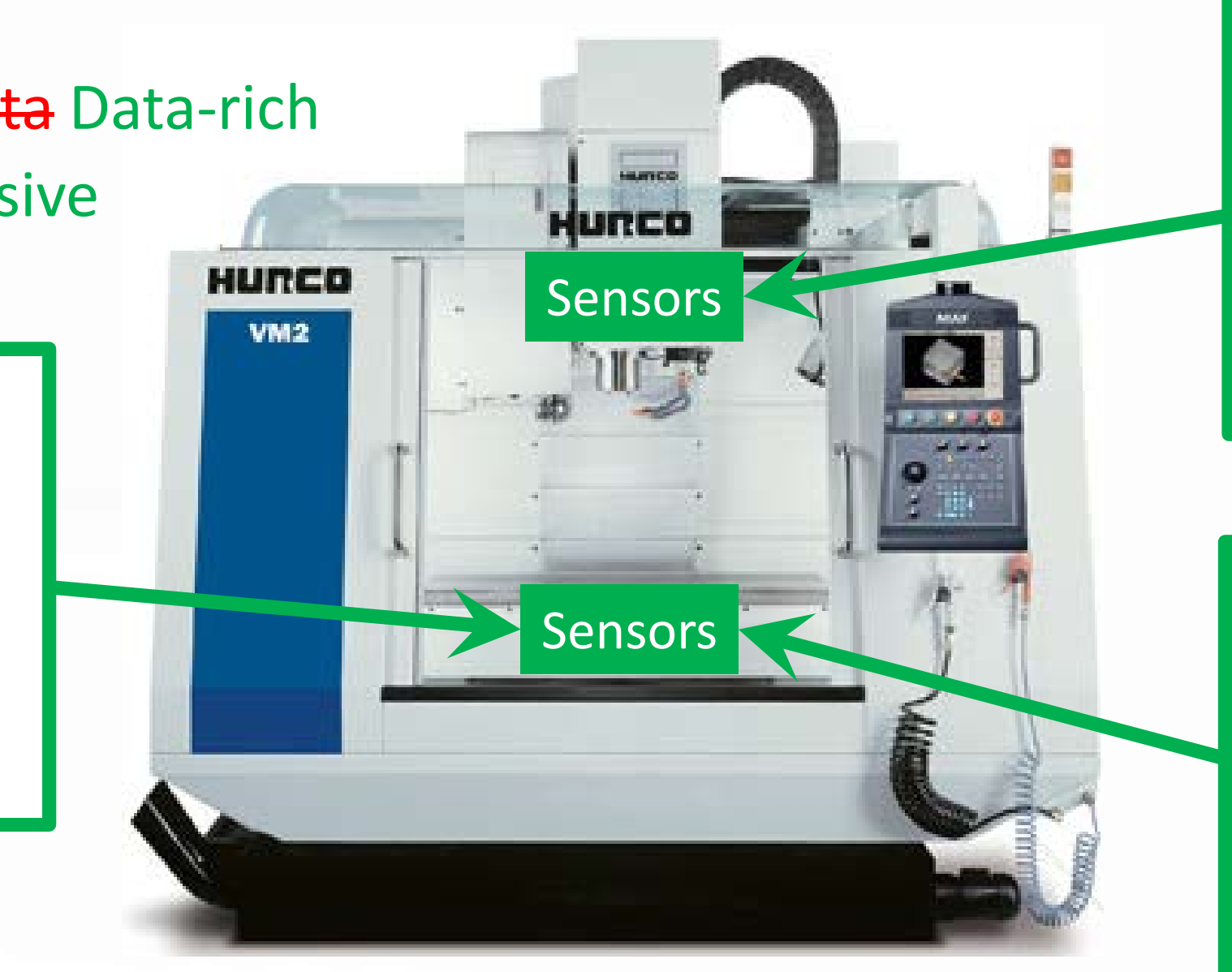

Spindle Health

Tracking

[How?]

Linear Axis Health Tracking

[How?]

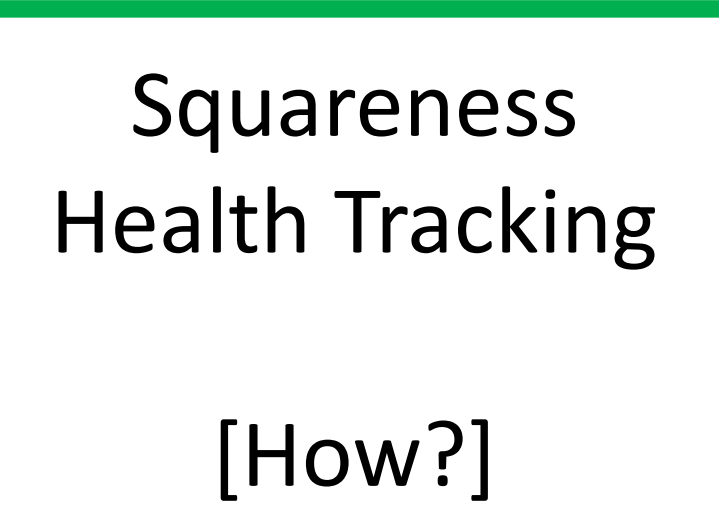




\section{GOAL: Smart Machine Tools}

- Make machine tools self-aware with diagnostics of performance \& root causes

- Predict part errors based on health tracking \& optimize asset management

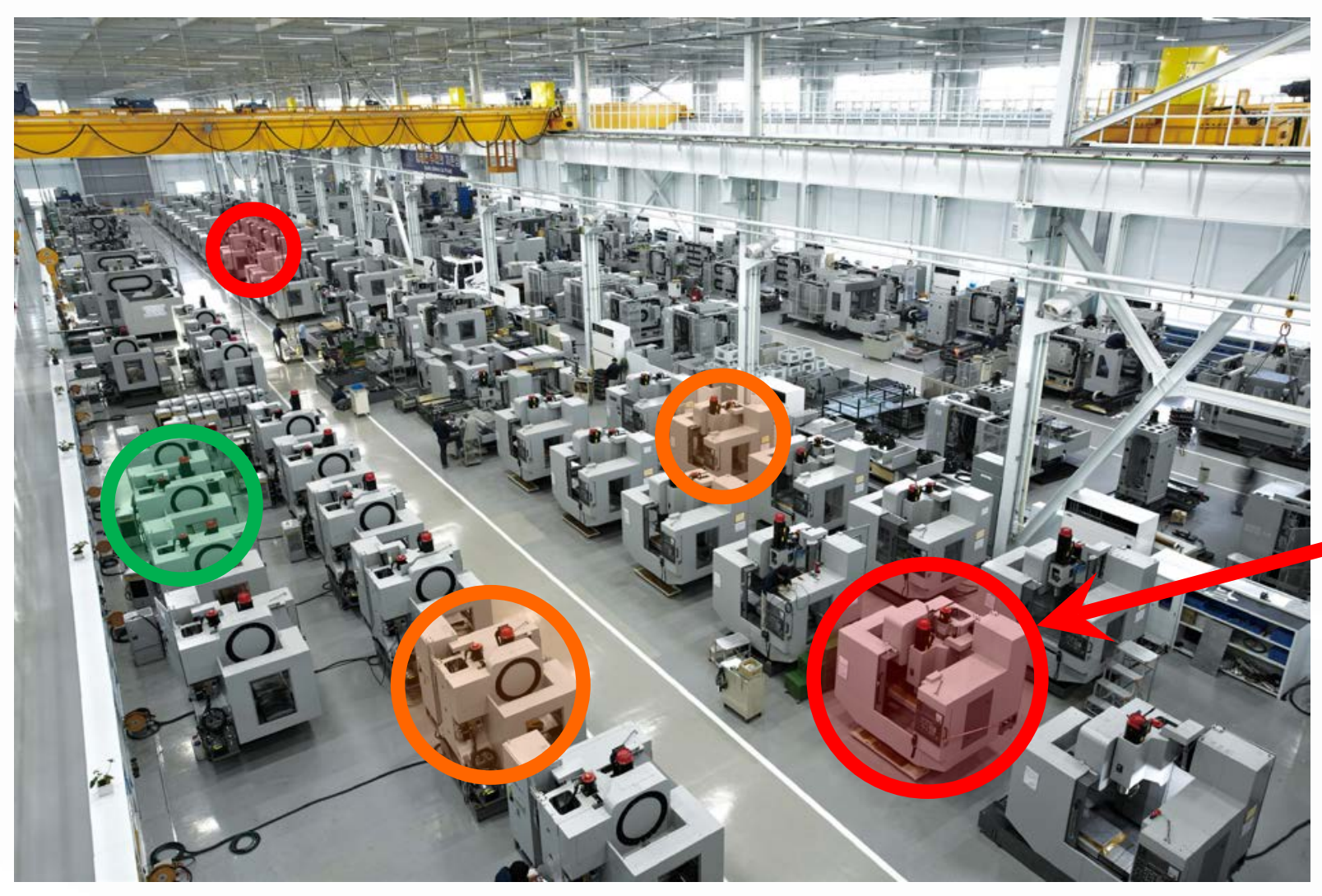

Tablet

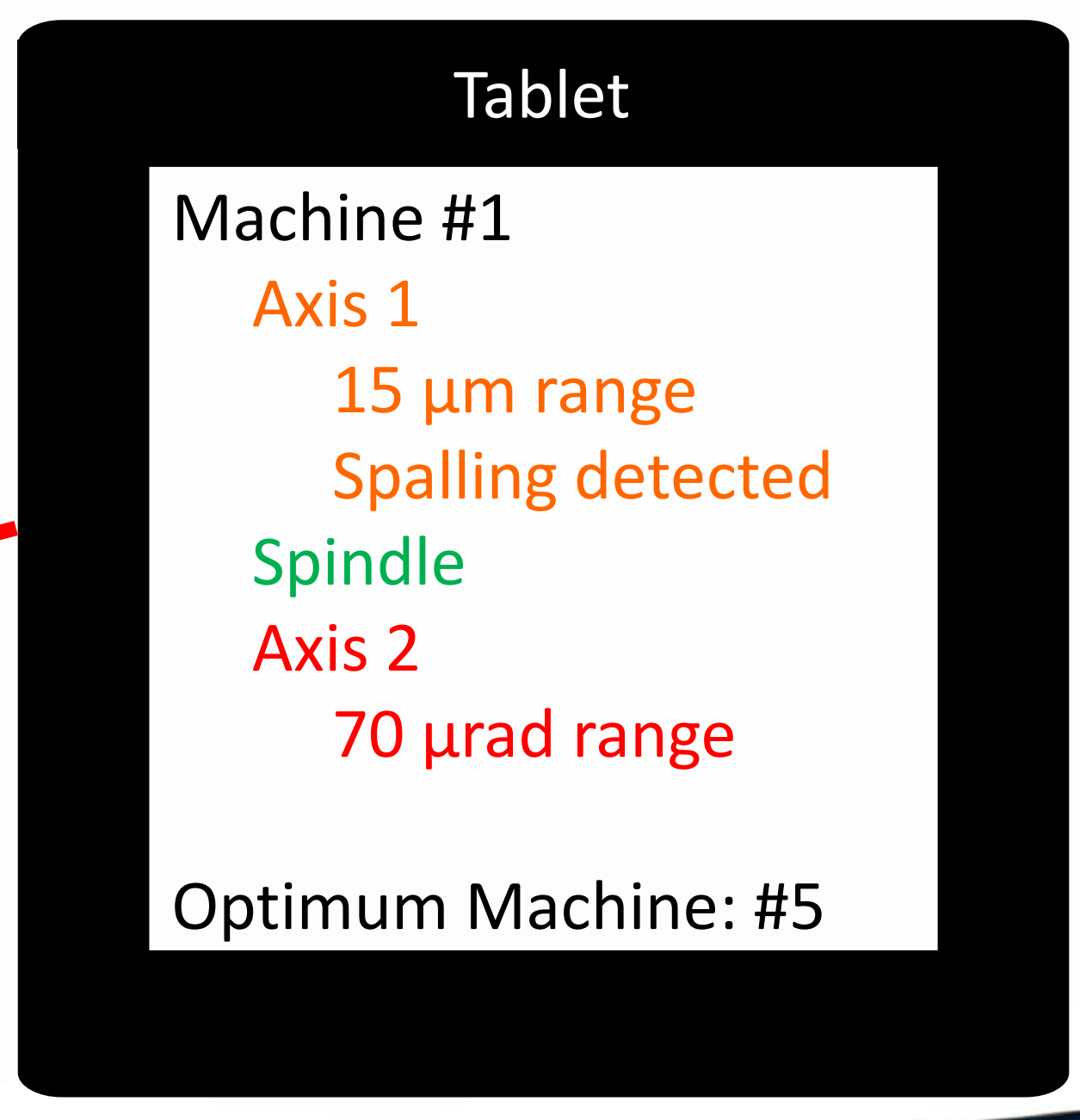




\section{IMU for Linear Axis Monitoring}
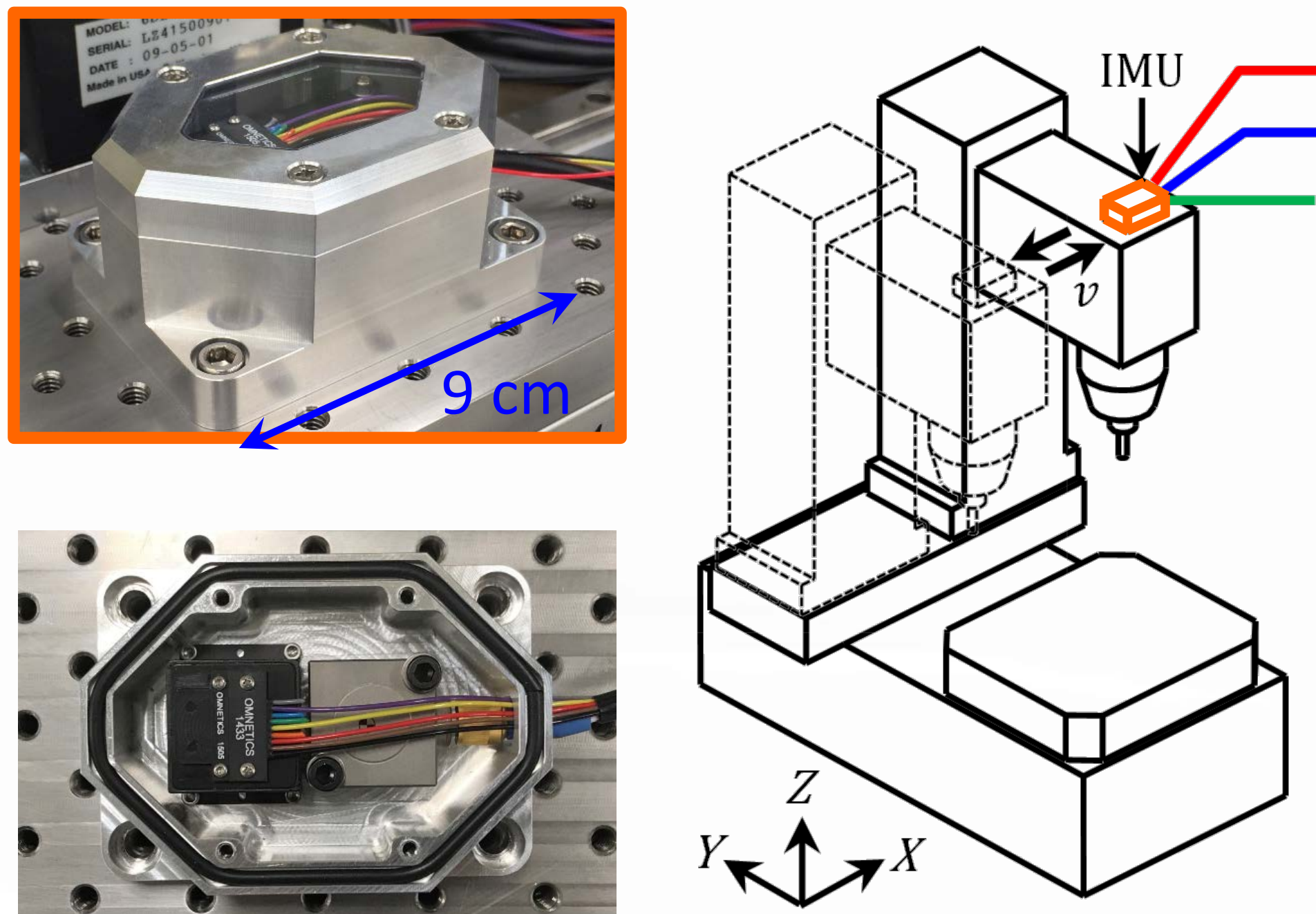

Fast Speed Data Moderate Speed Data Slow Speed Data Data Fusion with Accelerometer (A) and Rate Gyroscope (RG) Data

Translational Motion $\underset{0}{\stackrel{\text { Spatial Frequency }}{\mathrm{Shamana}} \mathrm{A}}$ Angular Motion Whamm $\longleftarrow$ option as available

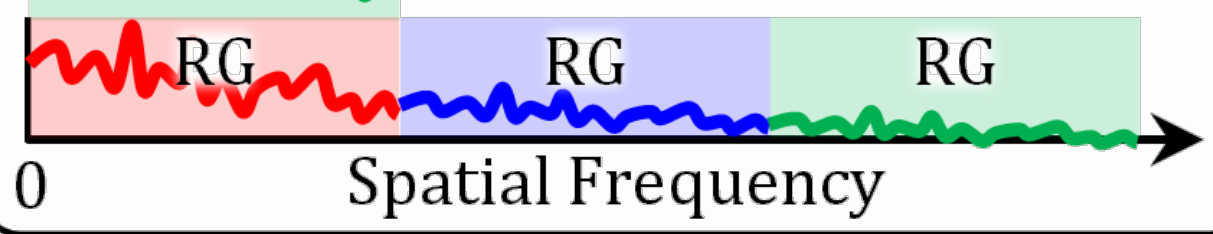




\section{IMU Data Collection}

- Each run uses 3 different axis speeds

- IMU can live within machine tool for usage with no setup

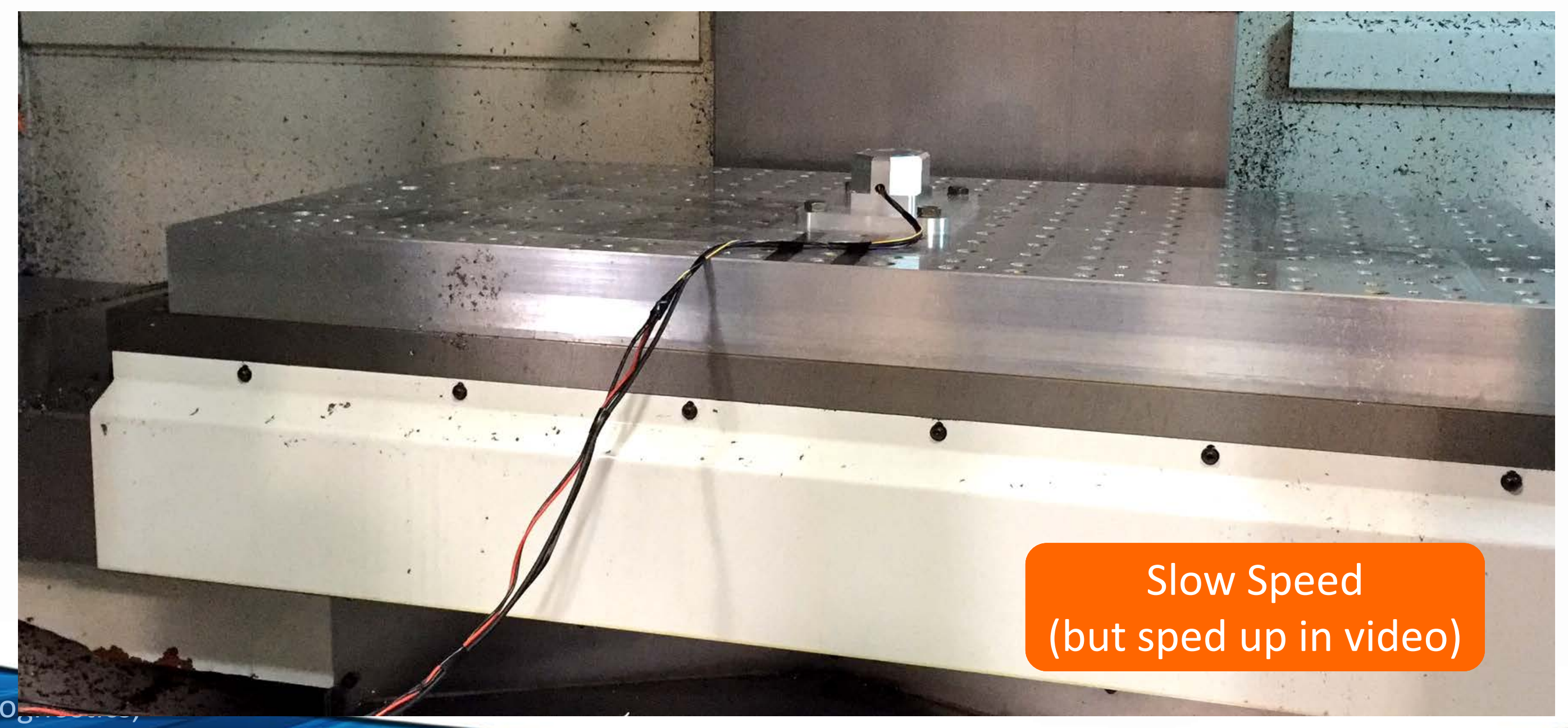




\section{NIST Linear Axis Testbed}

- Testbed to study IMU-based method \& diagnostics / root-cause analysis

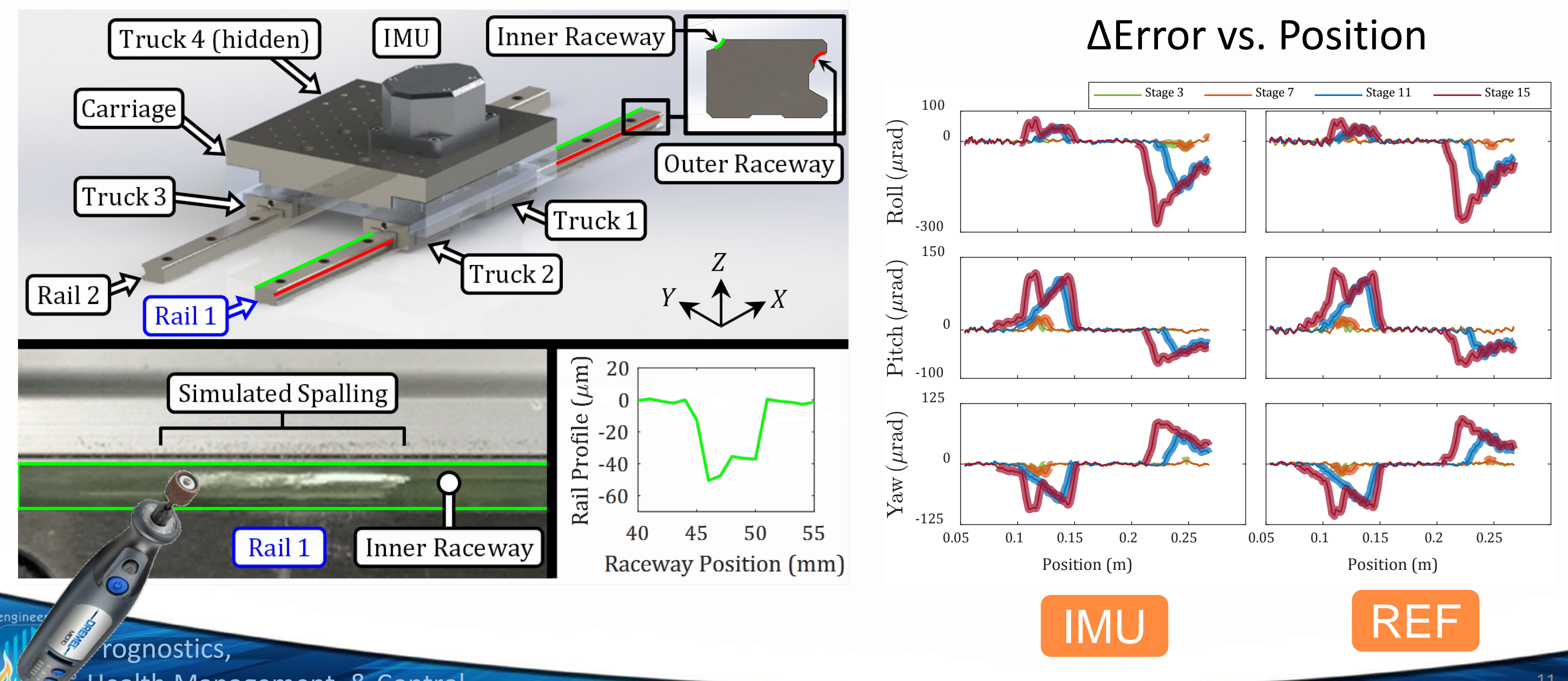




\section{NIST Linear Axis Testbed}

- Testbed to study IMU-based method \& diagnostics / root-cause analysis
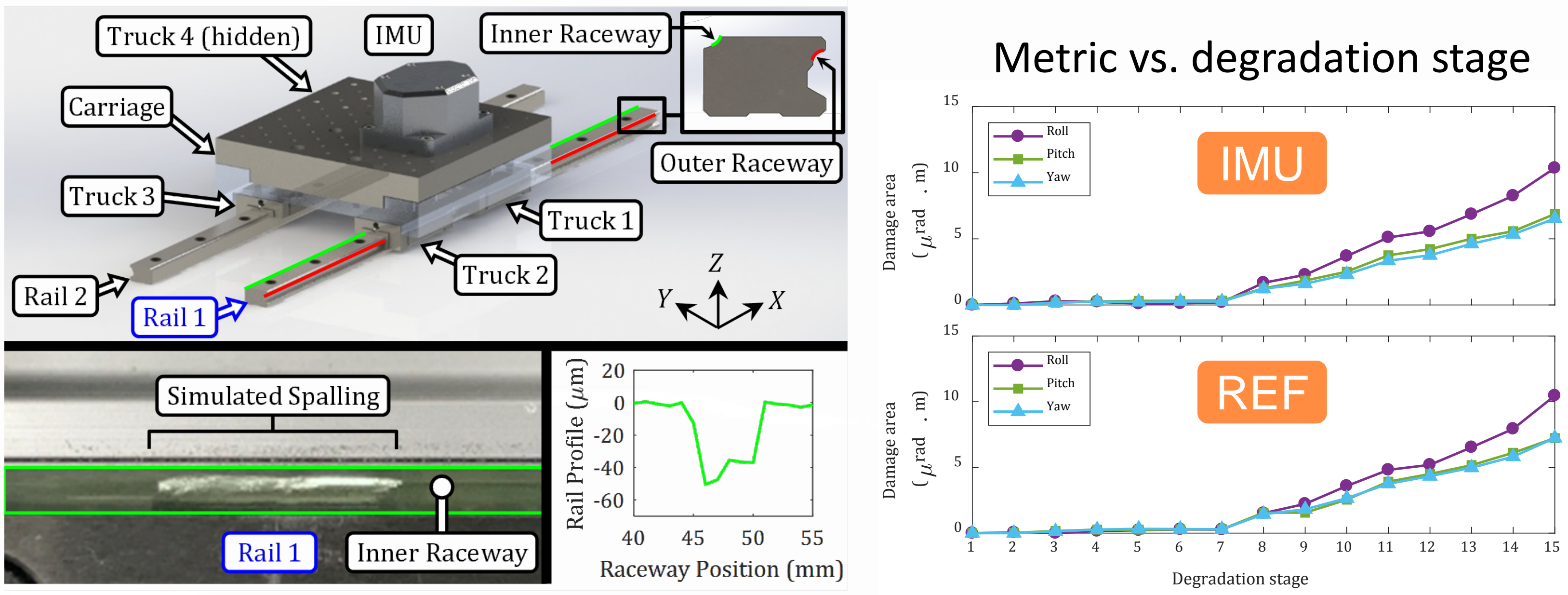


\section{Root-Cause Analysis for Rail Wear}

- Find root cause of changing error motions

- 4 possible physical causes: inner/outer raceway damage on Rail 1 or 2

- Root-cause analysis correctly identified spalling on inner raceway of Rail 1

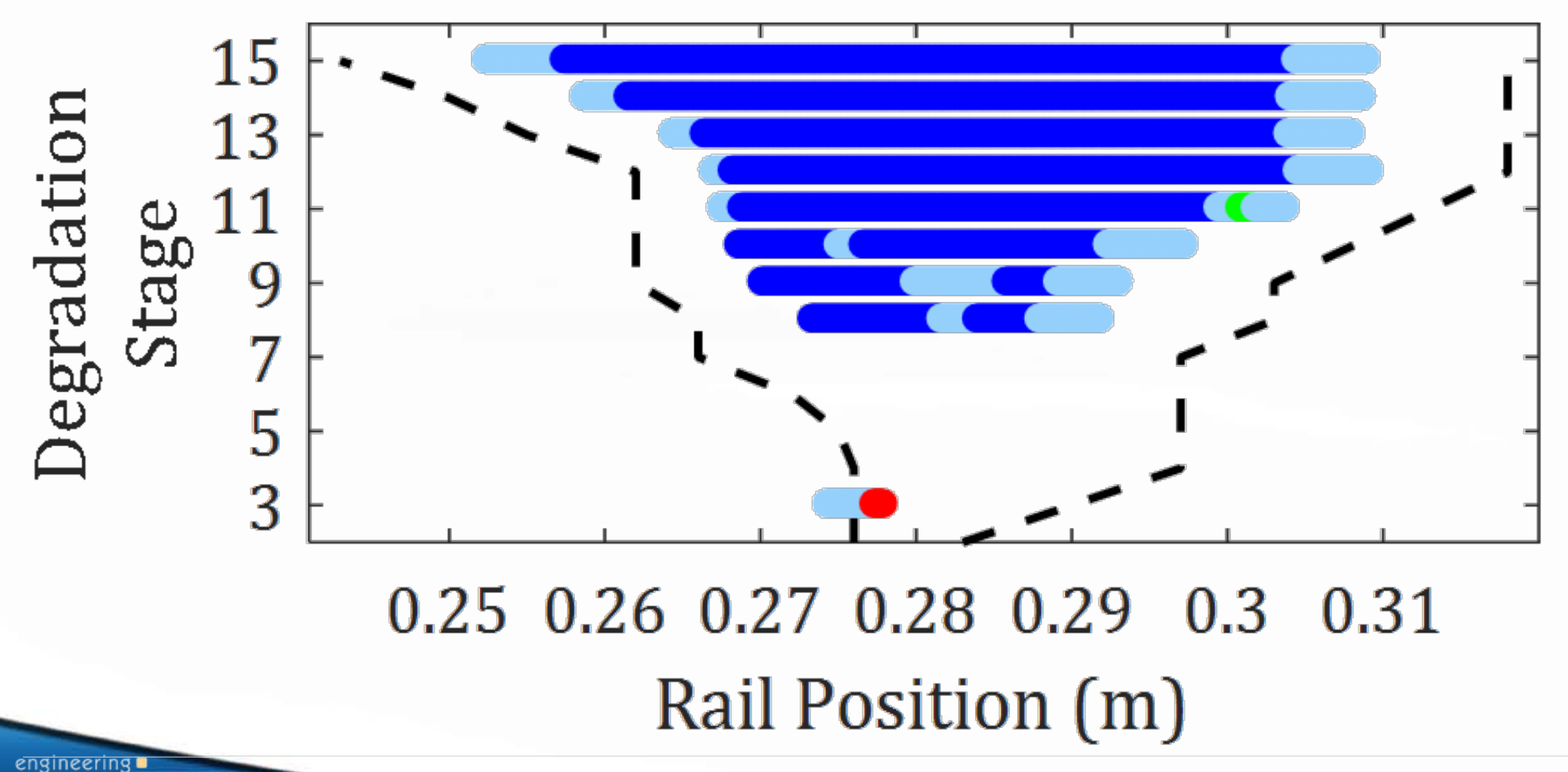

- Rail 1, Inner Raceway, $\mu=1$

- Rail 1, Inner Raceway, $\mu<1$

- Rail 1, Outer Raceway, $\mu<1$

- Rail 2, Inner Raceway, $\mu<1$

- Rail 2, Outer Raceway, $\mu<1$

$\begin{array}{lllllll}0.25 & 0.26 & 0.27 & 0.28 & 0.29 & 0.3 & 0.31\end{array}$

Rail Position (m) 


\section{IMU for Linear Axis Health Tracking}

- Research Opportunities to use IMU for Comprehensive Root-Cause Analysis

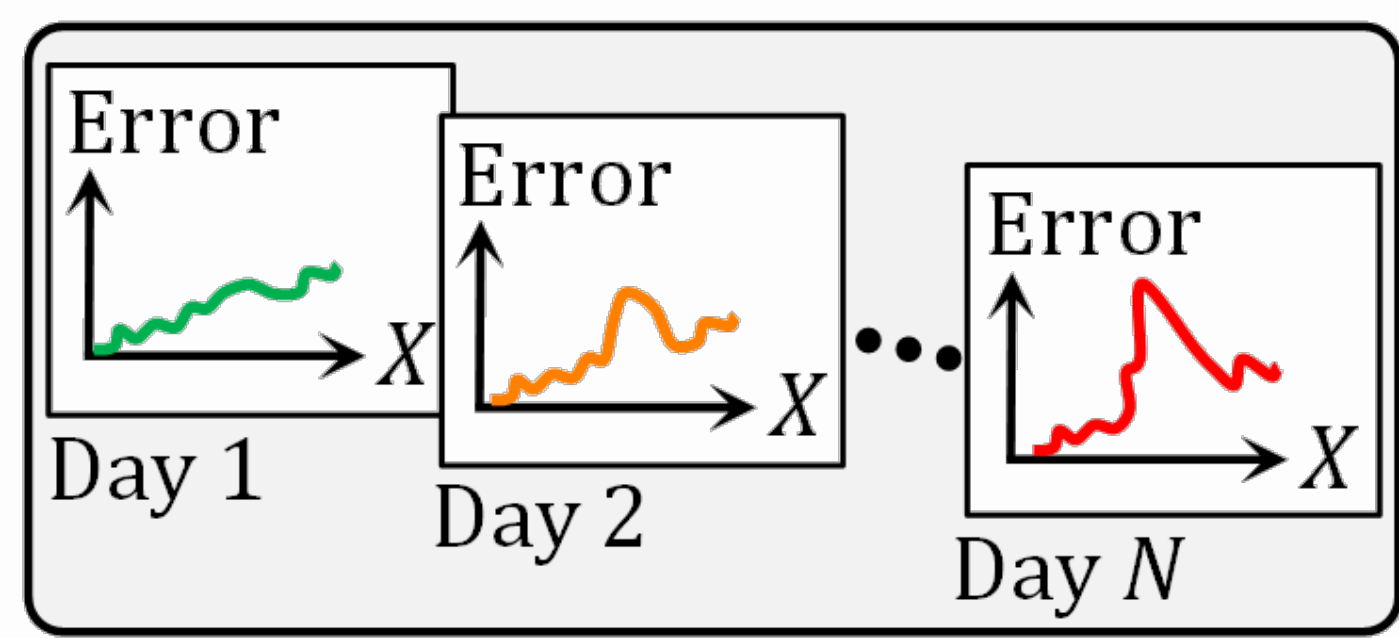

Error Motion Changes

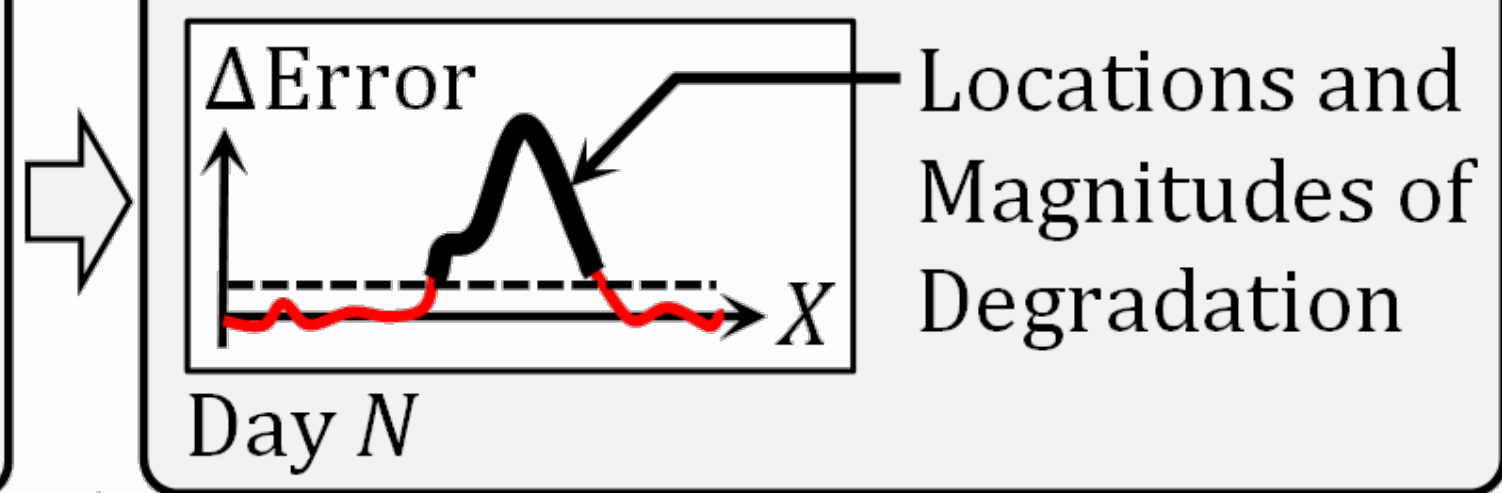

Day $N$

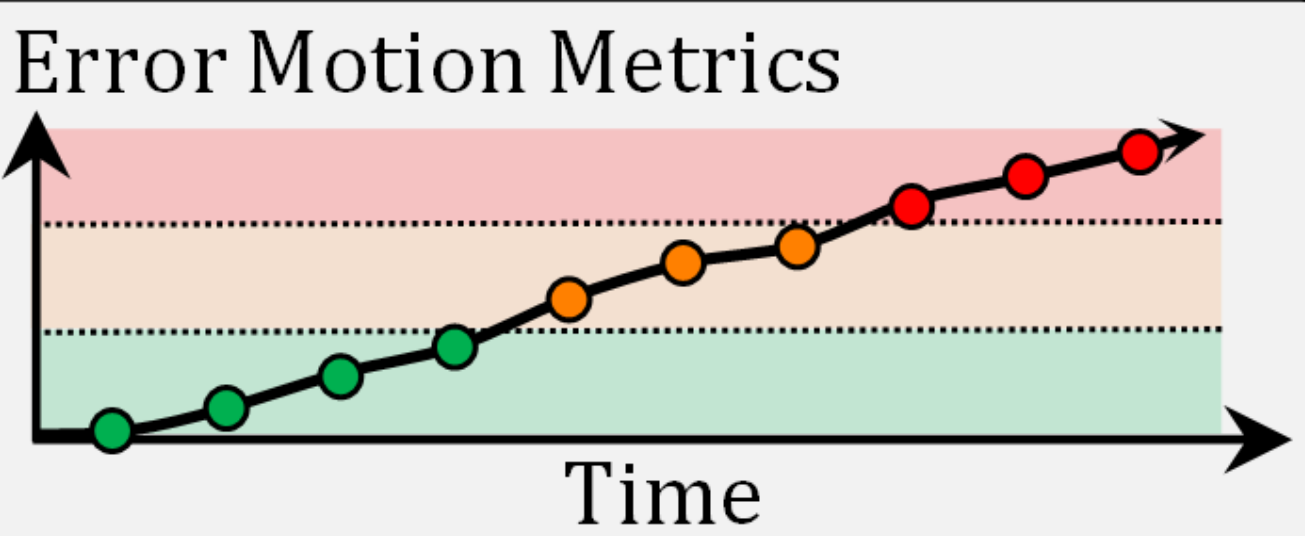

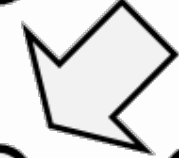

Root-Cause Analysis

$\checkmark$ Sources of Error $\rightarrow$ Trucks, Rails,

$\checkmark$ Lead Screw, Ball Nut, and/or Controlled Motor 


\section{GOAL: Smart Machine Tools}

- Industry challenge: "Machine health in 5 min?"

- On-machine measurement science to diagnose performance and root-causes

- Offline Online

- Lack of periodic data Data-rich

- Expensive Inexpensive

Linear Axis Health Tracking

[How?] IMU

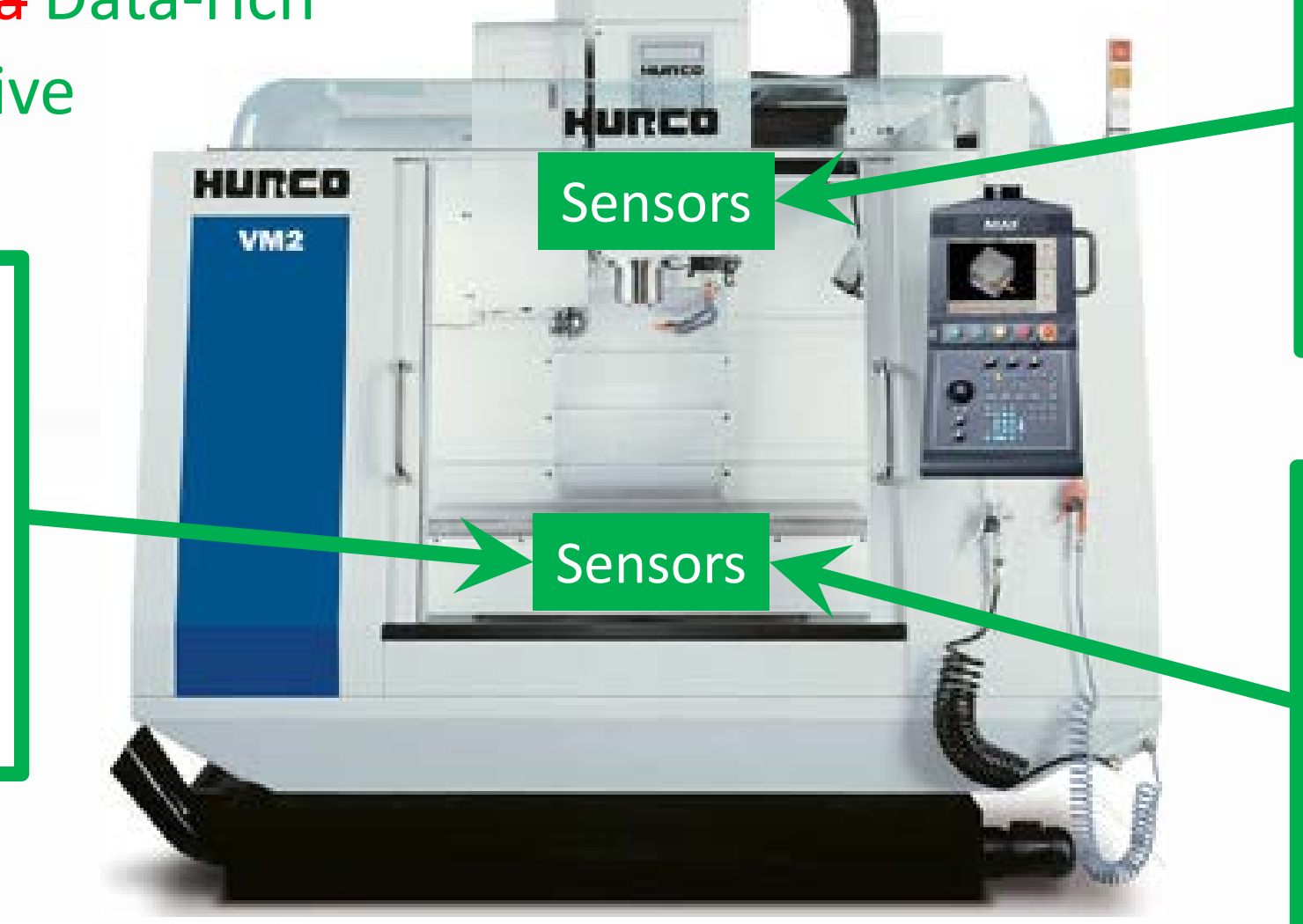

Spindle Health

Tracking

[How?] TBD

Squareness Health Tracking

[How?] IMU 


\section{Lesson \#1 - Smart \& Metrological}

- Traceable - Data is traceable to NIST

- Sensors calibrated along "measurement chain" to NIST

- Dimensional - Results are physical quantities

- Inspired by international machining

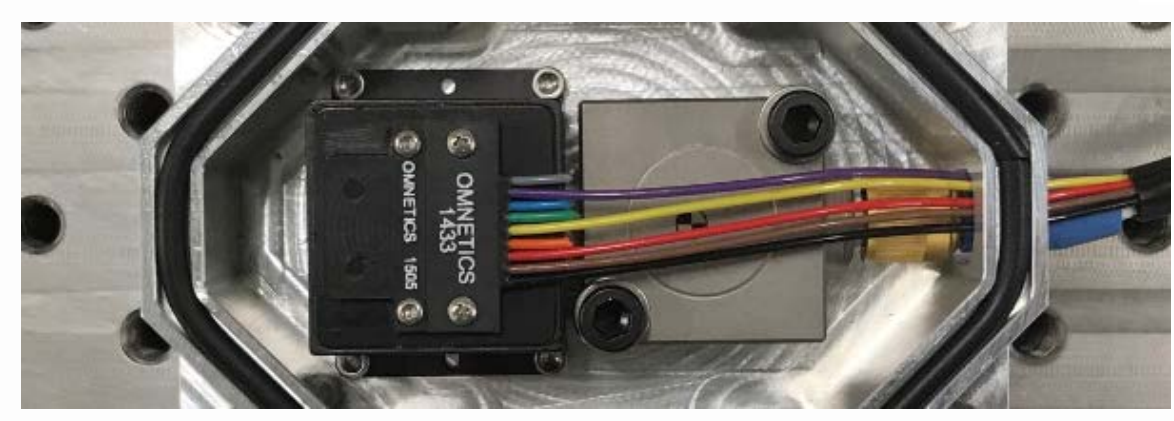
standards

- Tracking $\Delta$ error motion $>2 \mu \mathrm{m}$ and $>6 \mu \mathrm{rad}$

- Physical quantities can be measured

- Verify and validate - If possible!

- Compare results to those from traceable independent reference

- Even complicated diagnostics can be shown to be correct

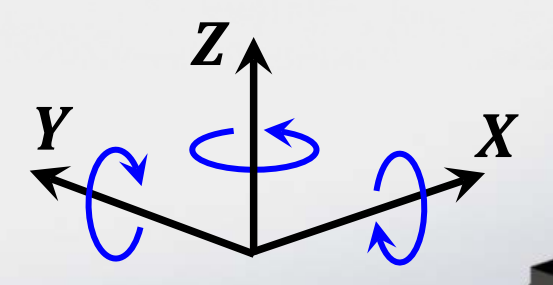




\section{Lesson \#2 - Smart \& Simple}

- Simple analytics

- Can be explained and standardized

- More robust because tested more easily

- Easier to implement for great adoption

- Goal- or physics-based thresholds

- Simple user setup

- Plug and play solutions

- Vendor neutral for flexibility
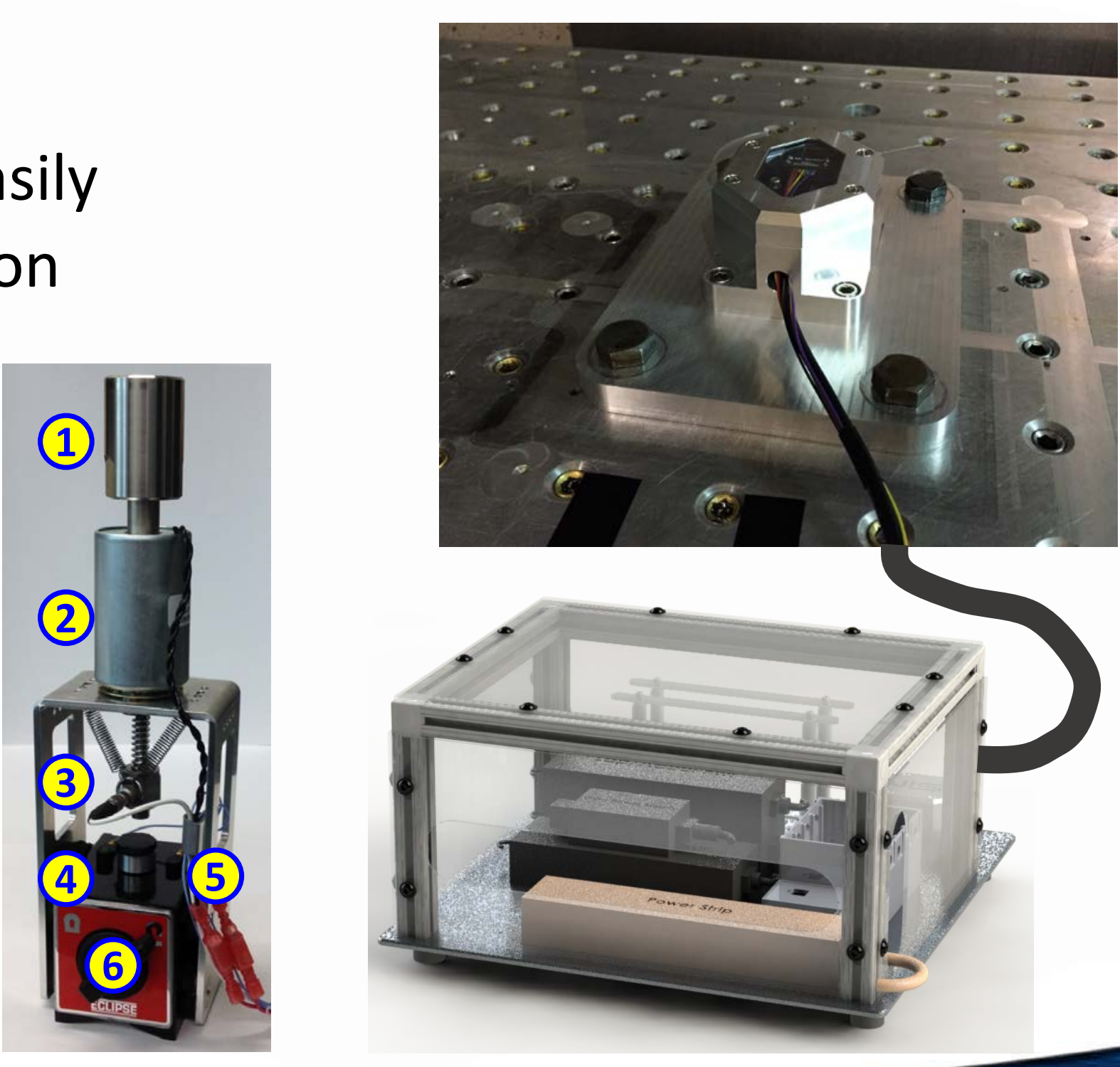


\section{Lesson \#3 - Future Directions}

- Make smart machine tools with online, data-rich, and inexpensive diagnostics $\&$ prognostics of performance \& root causes of faults/failures

- Predict part errors based on health tracking \& optimize asset management
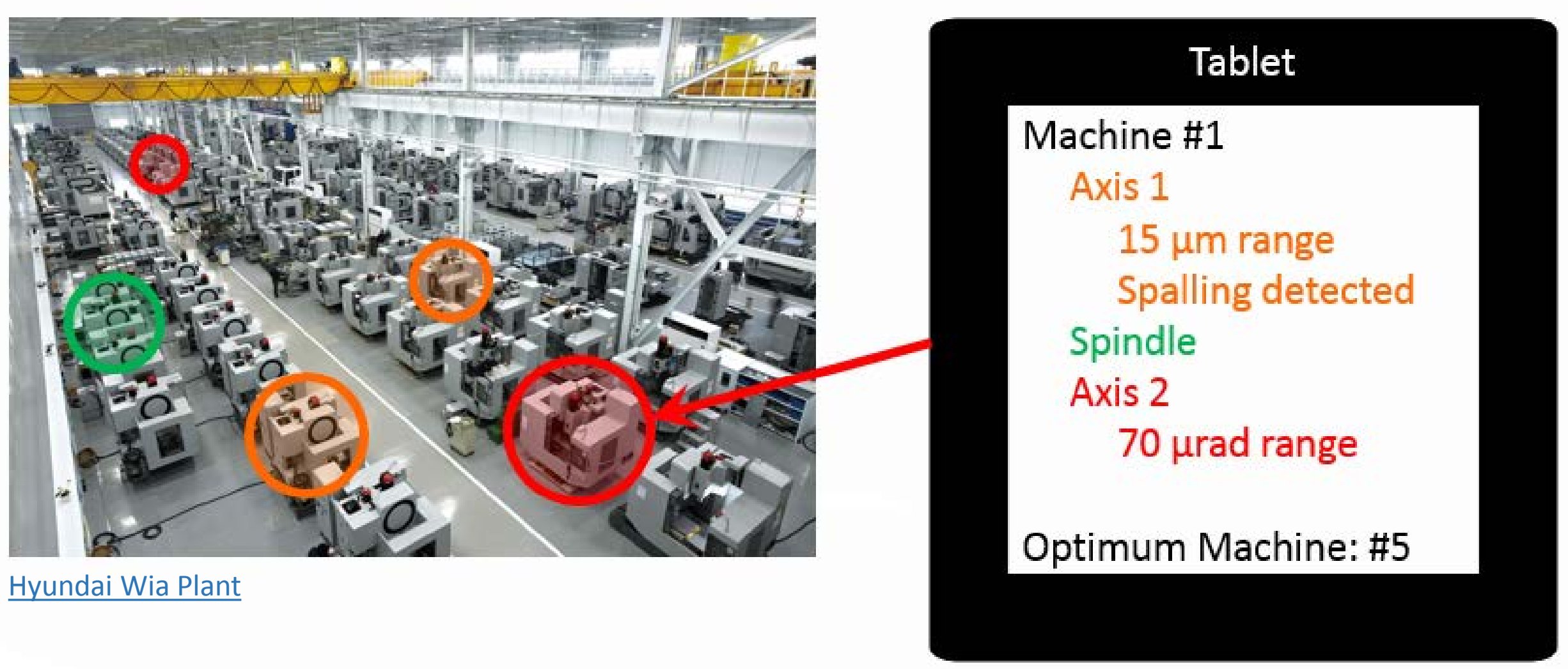


\section{Gregory W. Vogl}

Intelligent Systems Division gvogl@nist.gov (301) 975-3198

\section{NIST}

100 Bureau Drive, MS 8230 Gaithersburg, MD 20899

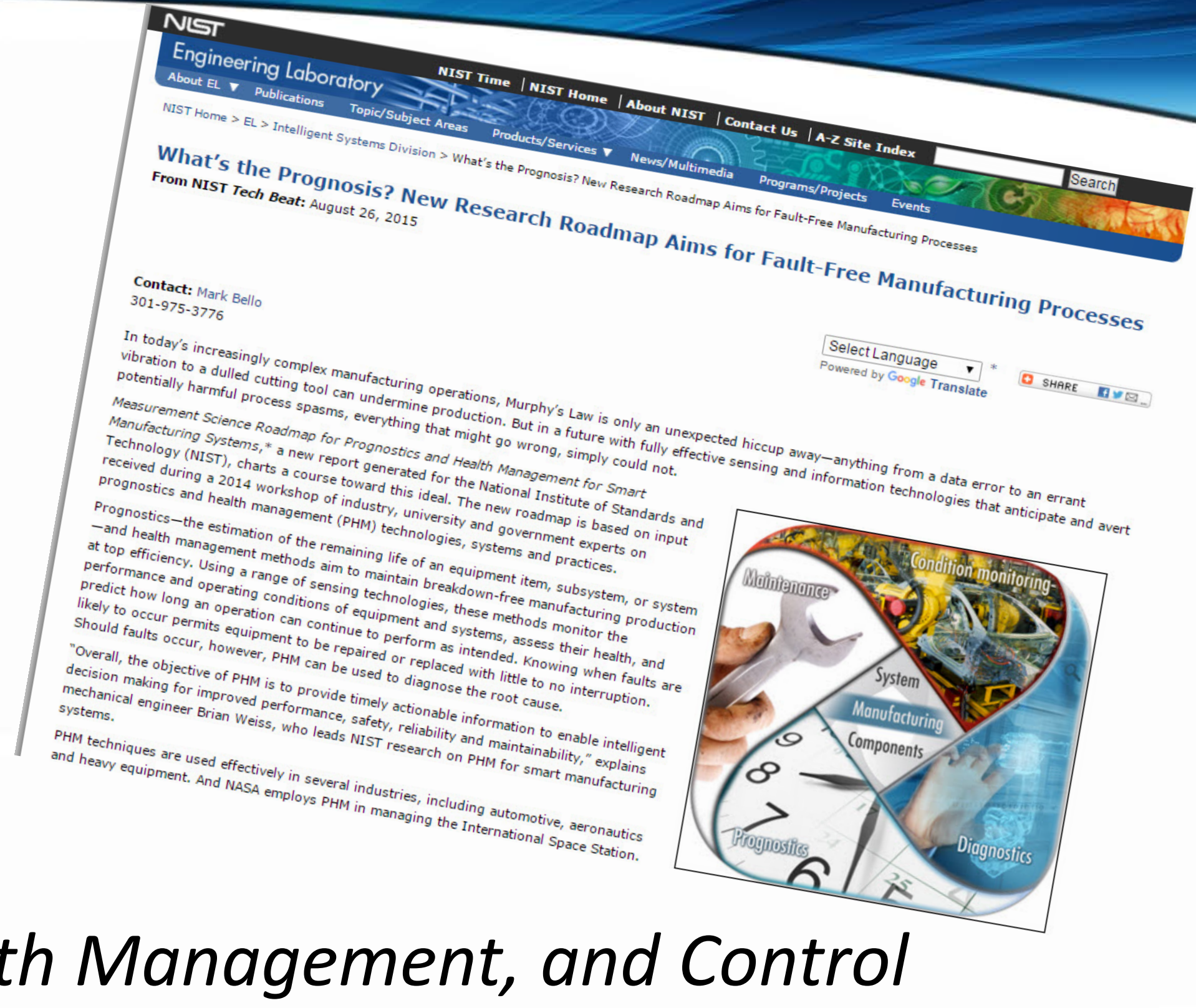

\section{Prognostics, Health Management, and Control}




\title{
Connecting and Deploying Smart Manufacturing Technology to Support PHM
}

\author{
Moneer Helu \\ Engineering Laboratory \\ National Institute of Standards and Technology
}

NIST Industry Forum

May 8-11, 2018 | Gaithersburg, MD 


\section{Disclaimer}

- Identification of commercial systems does not imply recommendation or endorsement by NIST

- Identified commercial systems are not necessarily the best available for the purpose 


\section{The Digital Thread Concept}
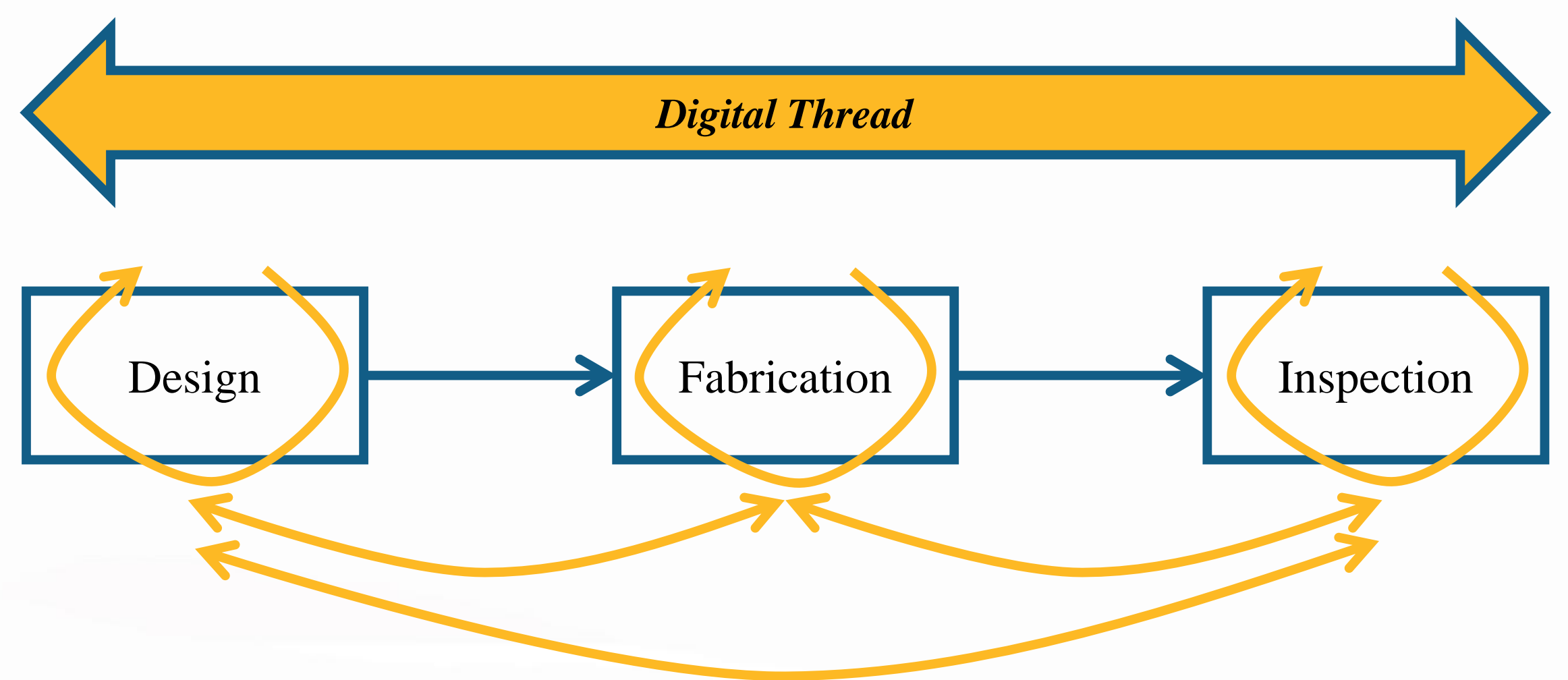

Information sharing across the digital thread can improve the overall performance of the product design and manufacturing process 


\section{Lifecycle Information Framework}

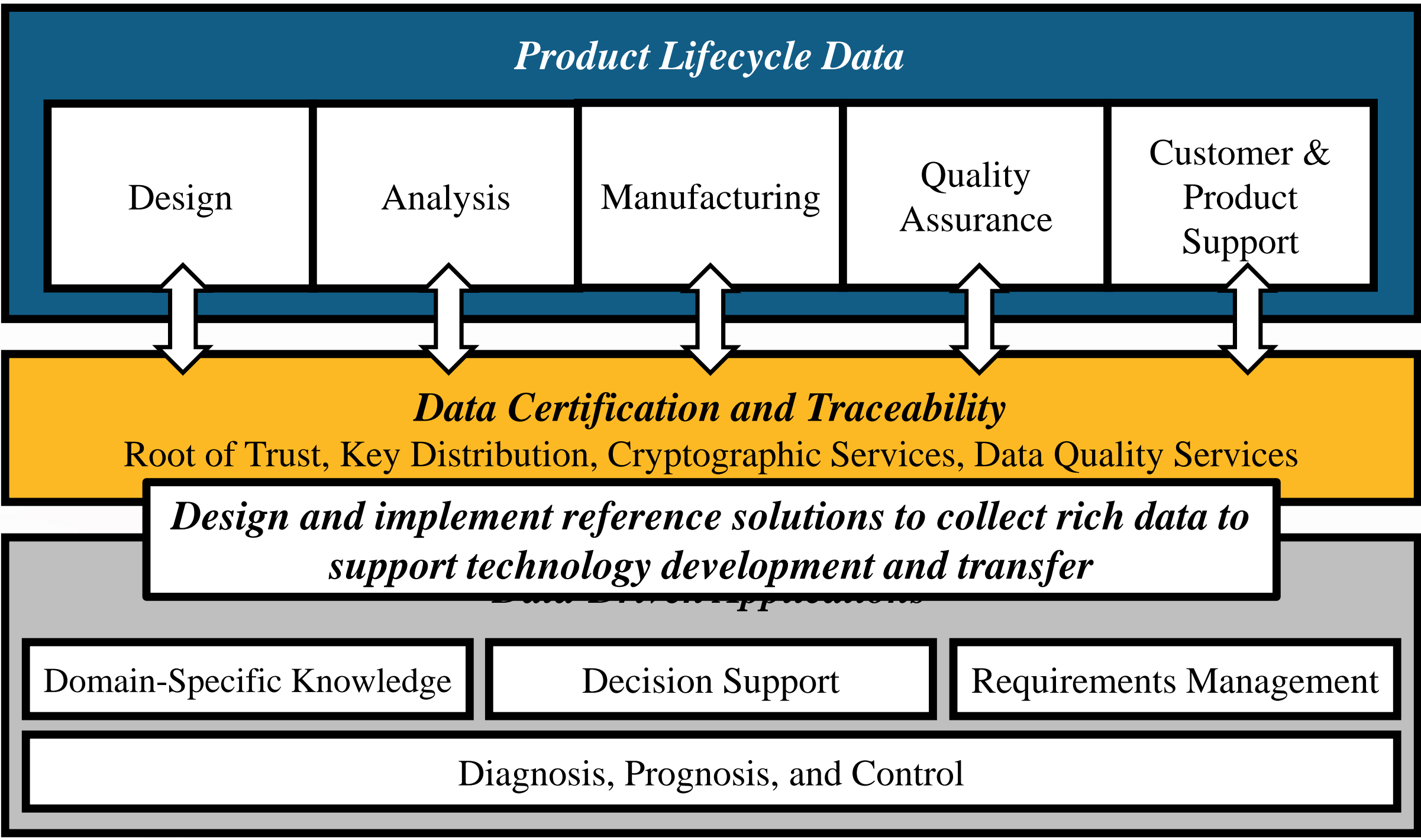




\section{Current Challenge}

- PLM solutions:

- CAx: CAD, CAE, CAM, etc.

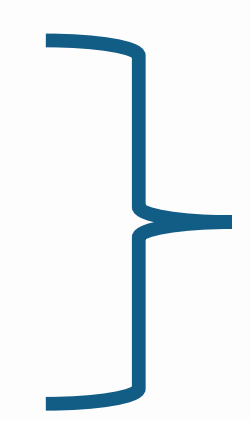

Primarily IT;

- PDM

- V\&V

Engineering focused;

Relatively expensive

- Operations solutions:

- Devices, SCADA, PLC

- MES, MOM

- ERP

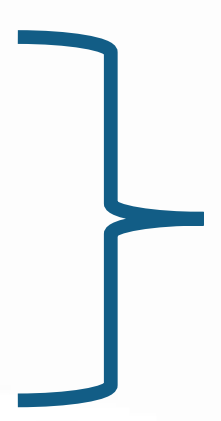

Mixture of IT and OT; Lack of integration across control levels

Integration of heterogeneous solutions across the product lifecycle for SMEs and larger organizations 


\section{NIST Smart Mfg. Systems Test Bed}

- Reference architecture and implementation

- Rich source of data for fundamental research

- Physical infrastructure for standards and technology development

- Demonstration test cases for education

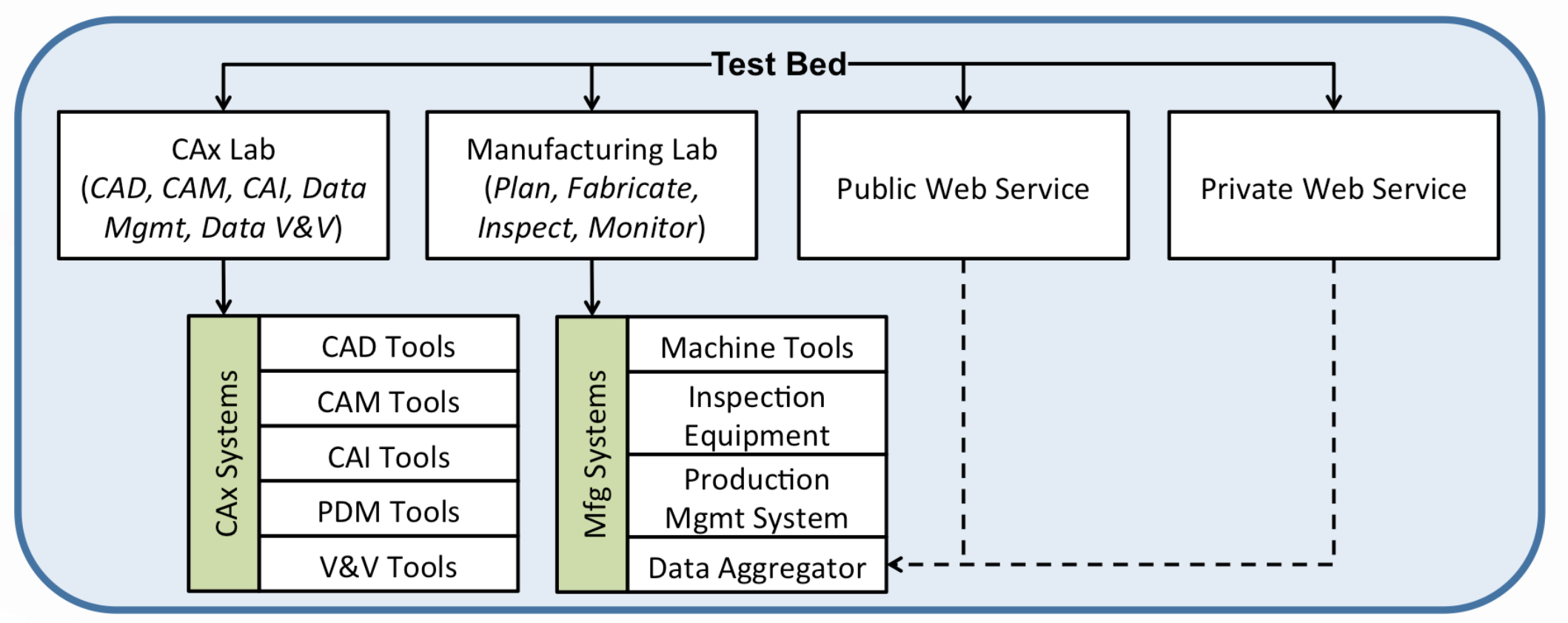




\section{Data Collection and Aggregation}

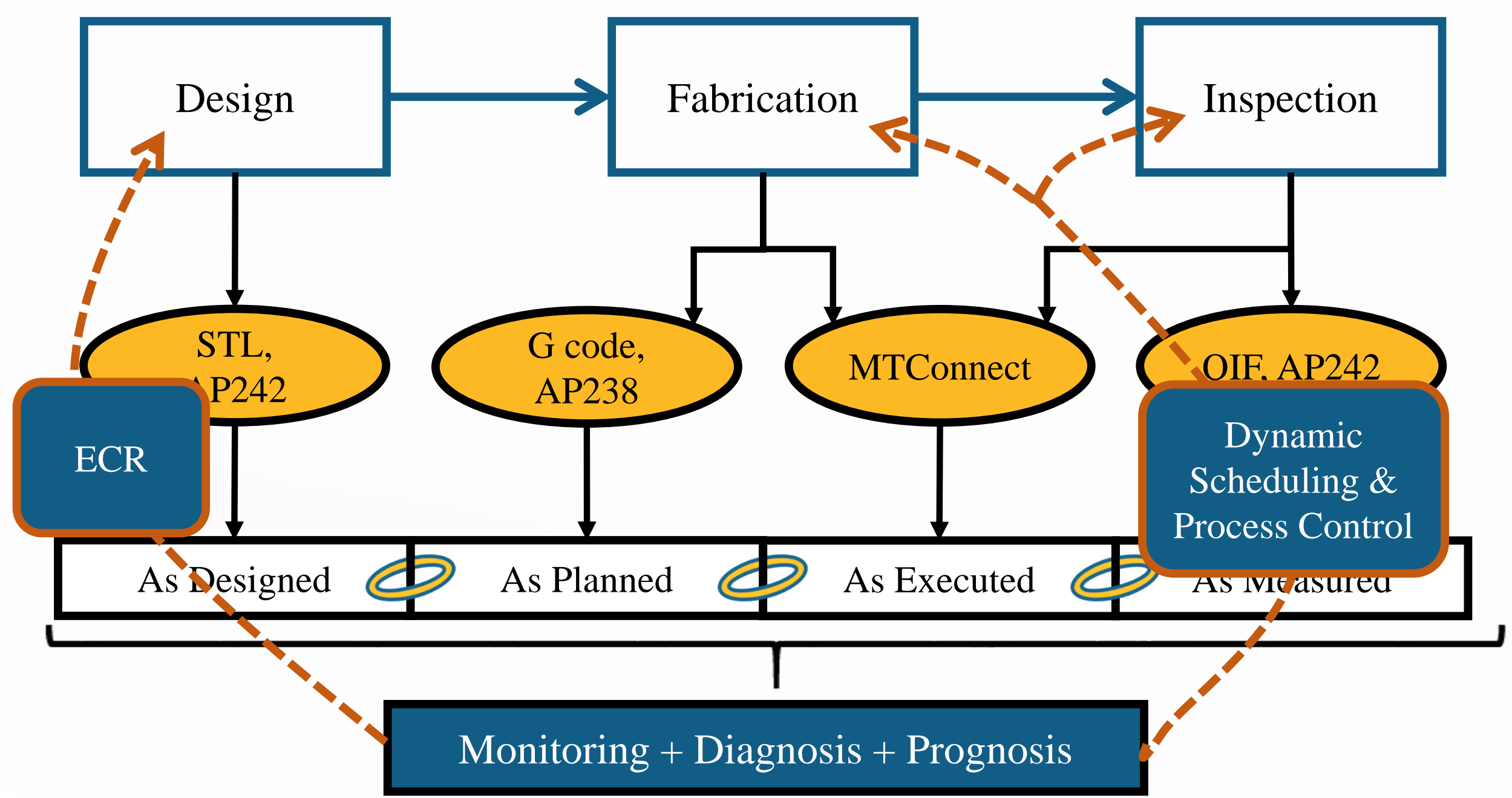

M. Helu, T. Hedberg (2015) Enabling Smart Manufacturing Research and Development using a Product Lifecycle Test Bed. Procedia Manufacturing, 1, 86-97. DOI:10.1016/j.promfg.2015.09.066. 


\section{Manufacturing Data Architecture}

- Designed as a four-tier architecture

- Implemented across three networks

- Provides segregated access to internal and external clients

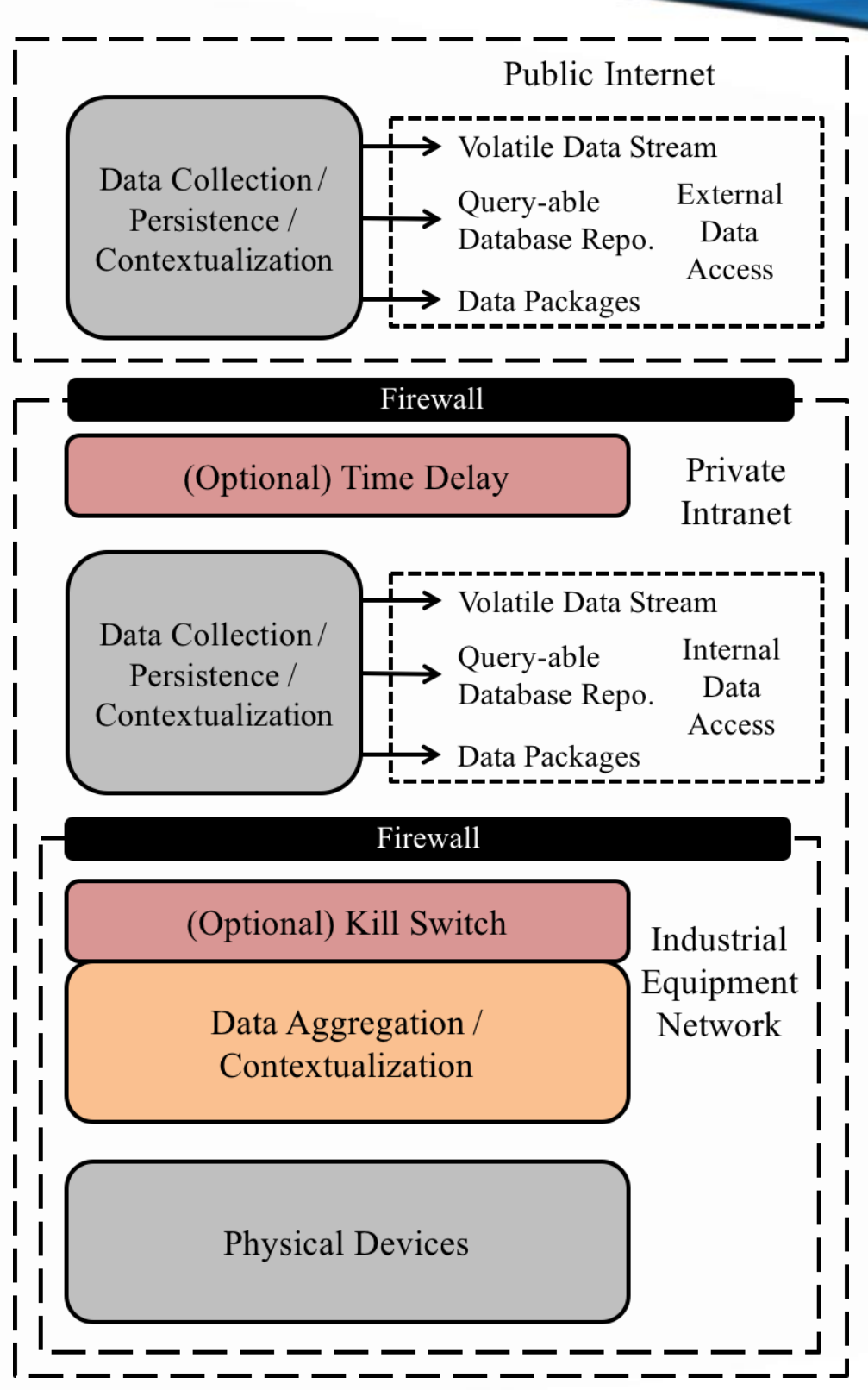




\section{Tier \#1: Services}

- Shop-floor IT and OT systems

- External sensors and equipment

- Any additional sources of data
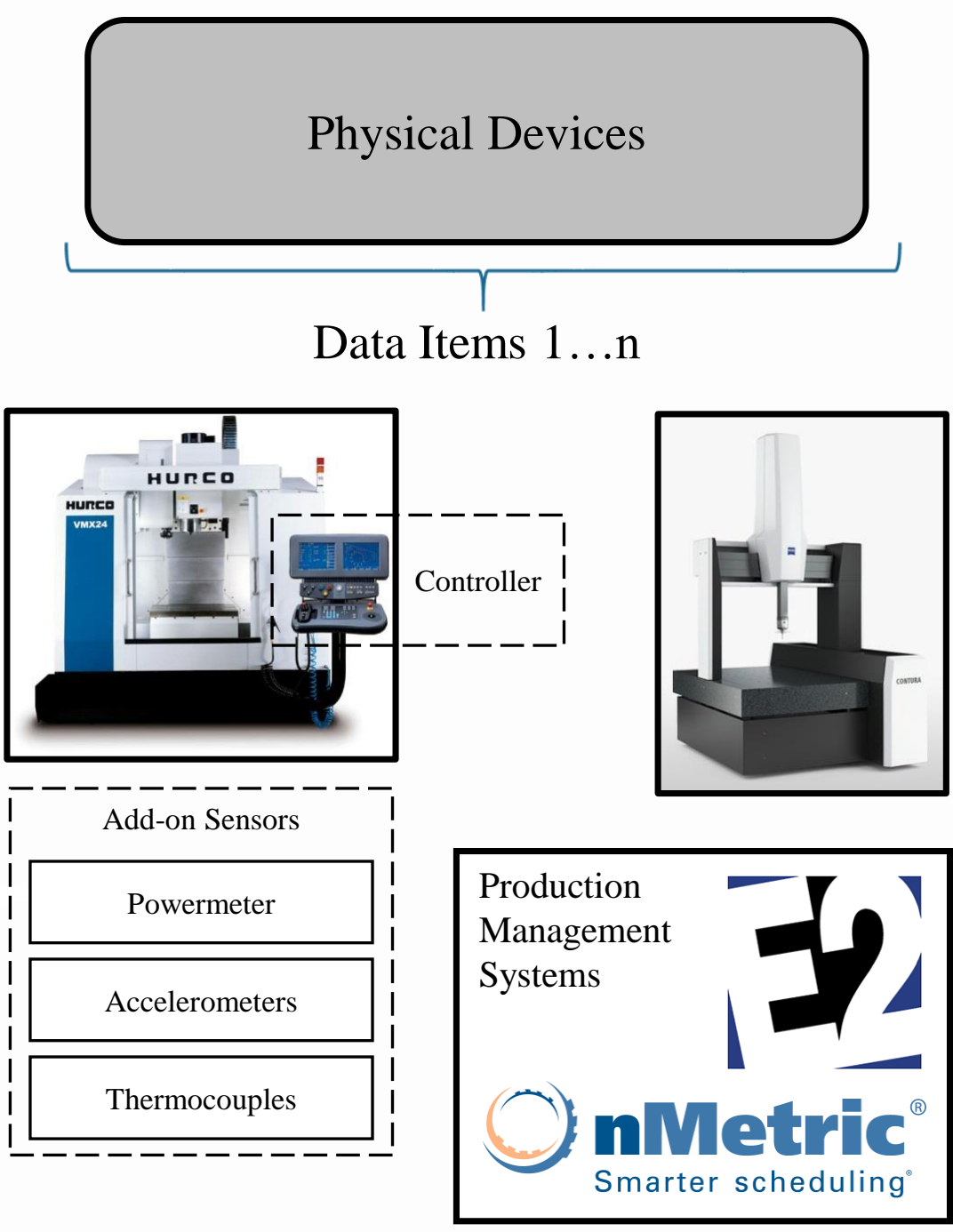


\section{Tier \#2: Aggregation}

- Aggregates and contextualizes service data

Data Aggregation /

Contextualization

- Provides data protocol translation

- Supplies data and information structure for underlying services

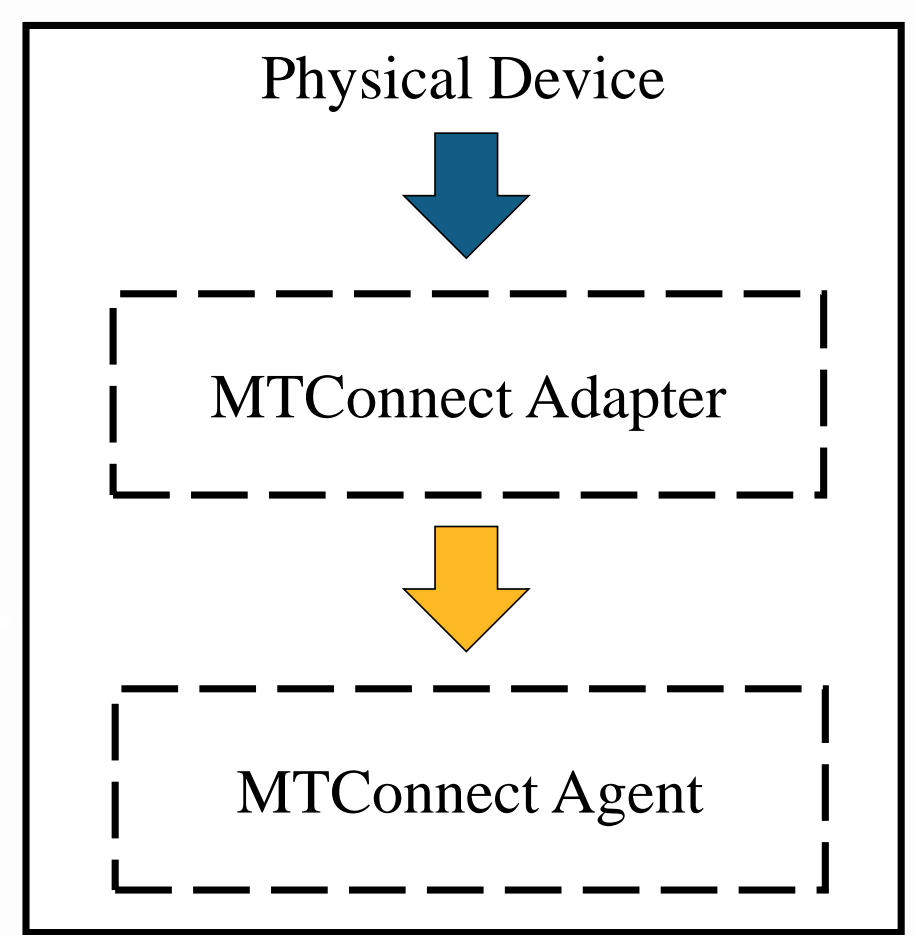




\section{Tier \#3: Delivery}

- Processes and contextualizes data for delivery to client

- Caches content for efficient performance

- Enables further development through data analytics

Data Collection /

Persistence /

Contextualization

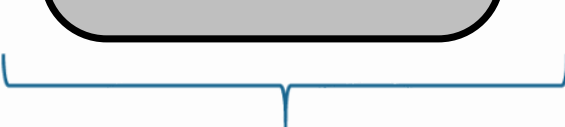

Devices $1 \ldots \mathrm{m}$

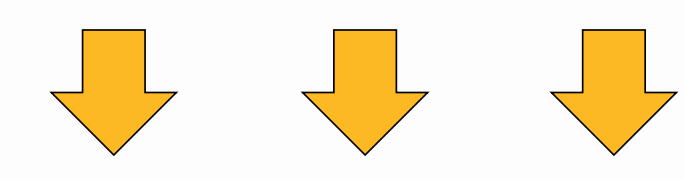

MTConnect Agent

Parsed XML

Documents 


\section{Tier \#4: Client}

- Responsible for data delivery

- Consists of web applications and clients

Access the SMS Test Bed at: https://smstestbed.nist.gov

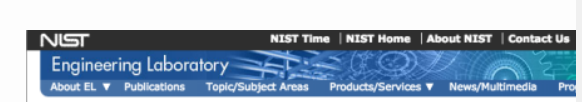

Smart Manufacturing Systems Test Bed

Volatile Data Stream

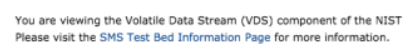

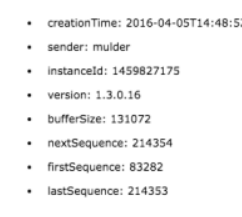

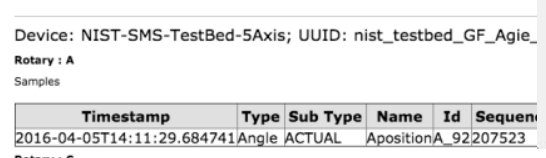

Volatile Data Stream

$\longrightarrow$ Query-able

Data

Database Repo. Access

Rotary
Samples

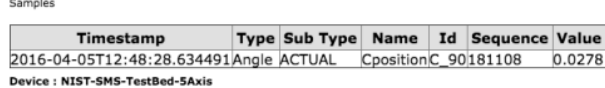

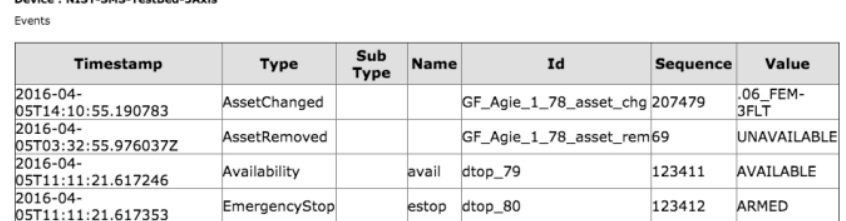

M. Helu, T. Hedberg, A. Barnard Feeney (2017) Reference Architecture to Integrate Heterogeneous Manufacturing Systems for the Digital Thread. J. Mfg. Sci. \& Tech., 19, 191-195. DOI:10.1016/j.cirpj.2017.04.002 
Data is the Gateway to further Insight...

Many manufacturers - especially SMEs believe that they understand their performance until confronted with real data 


\section{New Insights by Leveraging Manufacturing Data}

Refining information

- Detailed machine states

- Additional context to support correlation and diagnosis

- Additional context to support multiple viewpoints
Higher-Value Use Cases

- Predictive maintenance

- Prognostics

- Dynamic scheduling

- Business support (spare part provisions, RFPs)

- Workforce augmentation 


\section{Data Contextualization}

- Process of combining different types of data to provide a more complete perspective of some phenomenon

- Quality of information extracted from data depends on appropriateness of context developed during data curation

- Appropriate context depends on viewpoint 


\section{Viewpoints across the Product Lifecycle}

\begin{tabular}{ccc}
\hline Lifecycle Stage & Broad Focus & General Role \\
\hline Design & Features & $\begin{array}{c}\text { Define features to meet requirements of form, fit, and } \\
\text { function of part }\end{array}$ \\
\hline Planning & Capabilities & $\begin{array}{c}\text { Organize a set of capabilities executed through different } \\
\text { processes to create features of part }\end{array}$ \\
Manufacturing & Processes & $\begin{array}{c}\text { Implement processes with maximum productivity to } \\
\text { create features of part }\end{array}$ \\
\hline Inspection & Characteristics & Compare characteristics of manufactured feature to its \\
definition in design
\end{tabular}

(*) Context needed within each lifecycle stage may not be uniform

(*) Decision making tends to focus on one viewpoint in one lifecycle stage

(*) Decisions can impact larger portion of product lifecycle 


\section{Digital Technologies Provide Opportunity!}

- Growth and accessibility of IT in manufacturing:

- Smart manufacturing

- Digital manufacturing

- Cloud manufacturing

- Cyber-physical systems

- Internet of Things

- Industry 4.0

(1) Interoperability across enterprise and life cycle

(2) Generation of actionable intelligence

(3) Decision-making support

- New opportunities to advance manufacturing:

- Improved productivity

- Ensured first-pass success

- Augmented workforce development

- Reduced costs

(1) Many solutions available

(2) Historically limited market penetration

$\Rightarrow$ Difficult to navigate breadth of options 


\section{Getting Started...}

- Define use case

- What are my requirements?

- Identify supported devices

\section{Connectivity is insufficient}

- What data do I have access to?

- Evaluate network infrastructure

- How can I access and manage that data?

\section{Understand what you hope to accomplish!}

- Execute integration activities

- Who will I need to support my goals? 
Define use case...

Long-term success is predicated on developing an appropriate data management plan that enables the query of curated, contextualized data collected from devices to support identified use cases 


\section{Example of Data Management Requirements}

- General description:

- Product functions

- User characteristics

- Operating environments

- Interfaces:

- User

- Hardware

- Software

- Communications
- Features:

- VDS and QDR

- Data curation

- System administration

- Others:

- Performance

- Reliability

- Availability

- Security

- Maintainability 


\section{Further Implementation Challenges}

- Different data formats and data and communications protocols

- Need for process-related information to provide full context

- Large variety of equipment age and computational power

- Obsolete operating systems

- Large data volumes over large range of temporal scales

- Demanding limitations of physical environment

- Need for extensive time synchronization 


\section{Example of Physical Implementation}

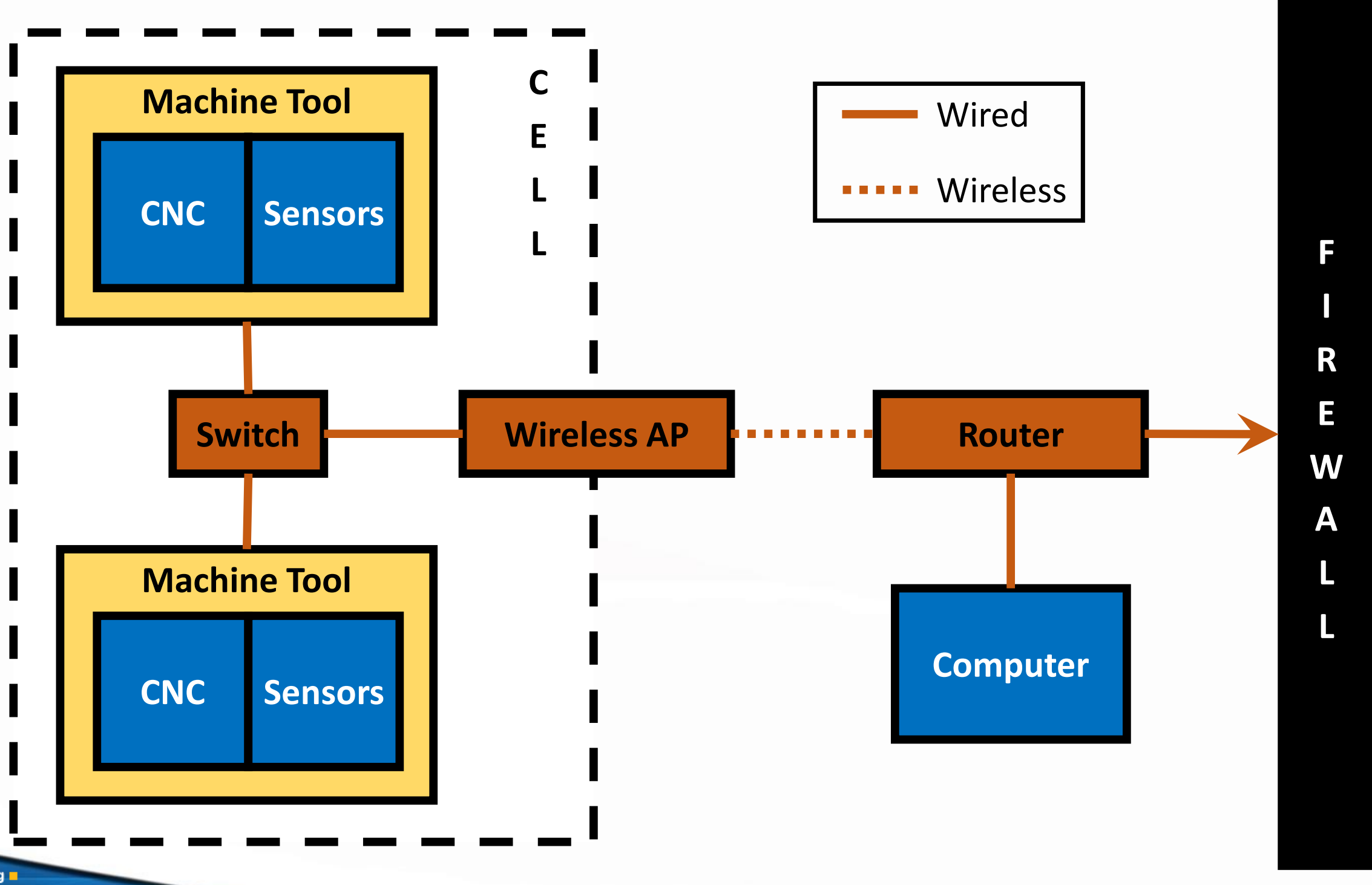

Private

Web

Services

\section{FIREWALL}

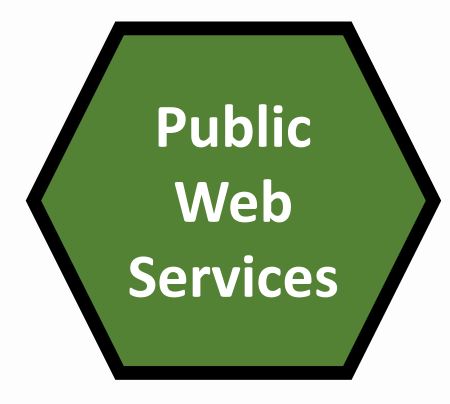




\section{Example Bill of Materials}

- (1x) Dell Precision T1650

- (1x) Cisco IR809 router

- (4x) Cisco Dual Radio 802.11AC AP POE

+ Machine Tool Upgrades

TOTAL \$20-\$25k

- (4x) Cisco SG100-24 24 Port Gigabit Switch

- (5x) Cisco SmartNet Service Contract (for router + APs)

- (2x) 1000-ft, 23-AWG CAT6 500 MHz UTP Solid, Riser Rater (CMR), Bulk Ethernet Bare Copper Cable

- (4x) CAT6 Plug Solid with Insert 50U, 100 pcs/bag

- (4x) RJ-45 Color-Coded Strain Relief Boots (50 pcs)

- (1x) Netgear FA411 16-Bit PCMCIA Network Card (10/100 Mbps)

- Various hardware items (e.g., double-sided tape, strain-relief tabs) 


\section{Additional Resources}

- General NIST SMS Test Bed Info: https://smstestbed.nist.gov/

- Documentation:

- Design and configuration of the smart manufacturing systems test bed: https://dx.doi.org/10.6028/NIST.AMS.200-1

- Reference architecture to integrate heterogeneous manufacturing systems for the digital thread: https://dx.doi.org/10.1016/j.cirpj.2017.04.002

- Software requirements specification to distribute manufacturing data: https://dx.doi.org/10.6028/NIST.AMS.300-2

- Email: smstestbed@nist.gov 


\section{Additional Resources}

- MTConnect:

- General information: http://www.mtconnect.org/

- Normative documentation: $h$ ttp://www.mtconnect.org/documents

- Informative resources: http://www.mtconnect.org/resources

- Open-source tools and demos: @ http://www.github.com/mtconnect

- Reference Agent: https://github.com/mtconnect/cppagent

- MTConnectR package for analysis of MTConnect data: https://cran.r-project.org/web/packages/mtconnectR/index.html

- STEP (ISO 10303-242): https://www.iso.org/standard/57620.html

- QIF: http://qifstandards.org/ 


\section{Thank you for your kind attention!}

\section{Moneer Helu}

Systems Integration Division

Engineering Laboratory

NIST

moneer.helu@nist.gov 


\title{
Using Unstructured Work Order Data to Improve Maintenance Procedures in Manufacturing
}

\author{
Michael P. Brundage
}

Systems Integration Division, Engineering Laboratory

National Institute of Standards and Technology

May 9, 2018 


\section{Outline}

1. Current Paradigm with Maintenance Work Orders (MWOs)

2. Transforming the MWO Data

3. Investigatory Analysis with MWO Data

4. Future Work 


\section{Current Maintenance Paradigm}

PHYSICAL PLANT

MAINTENANCE WORK ORDER

Date:

Requested by:

Building/Room:

Description of Needs:

Org. to be Charged:

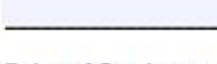

Estimated Cos Amount:

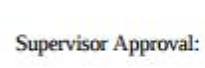

Date:

VP of Administration Approval:

Work Completed by:

Return completed form to Administrative Services
Rev 501
Date Mach

29-Jan-16

1-Jun-16

FT

1-Jun-16
Description

Issued By Date Up

ssued By Date Up Assigned
As

St\#14 tool detect INOP

Brakes worn -Not

stopping when in gear

St\#7 rotator collet

broken -wait for Bob B

to show him how to

remove

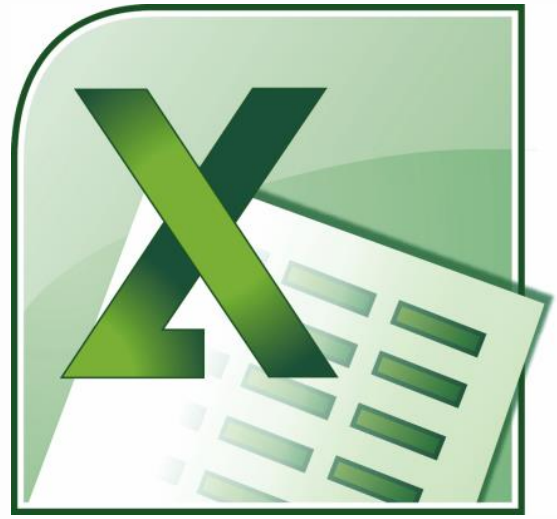

Resolution

Slug detector at station 14 not working. Would not recognize "Start" signal.
Repaired 


\section{Maintenance Work Order Data}

"Hydraulic return line replaced"
"Head removed and cleaned thoroughly. Found cam action spring binding on one tool station. Removed spring and cleaned up burring on spring; Reset and reinstalled"
"Turret removed, cleaned, reinstalled, and aligned"
"Bearings bad; removed spindle and replaced bearings"
"Retrieved motor from spare automation and installed" 


\section{Current Maintenance Paradigm}

- Expertise Driven

- Sensors not always present

- Often unstructured MWOs

- natural language; domain-specific abr. and jargon

- "tribal" knowledge

- Proprietary maintenance software 


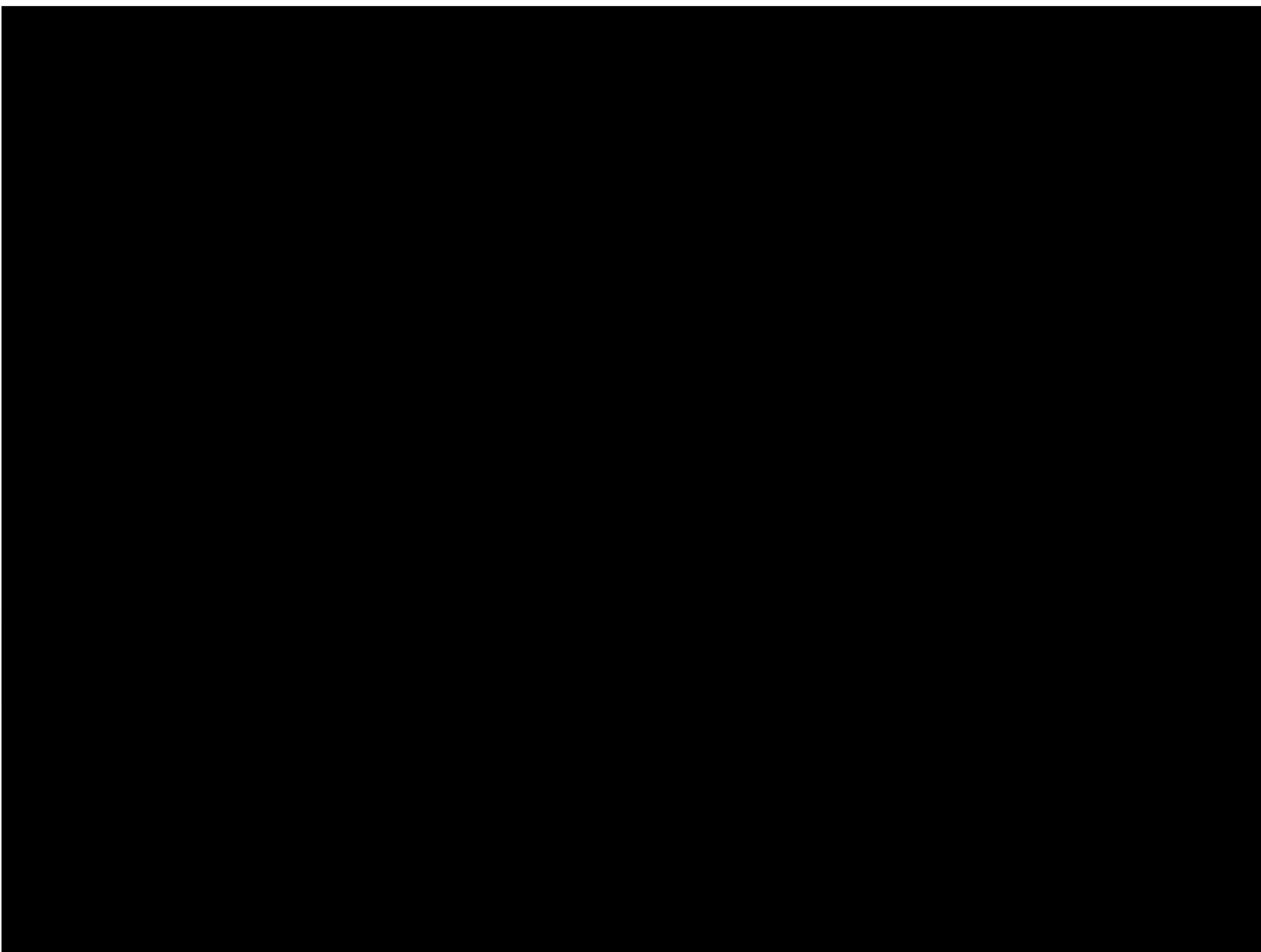

https://www.youtube.com/watch?v=jHbl_B2sPA0\&feature=youtu.be\&t=1m35s 


\section{Interactive Case Study}

"The cutting tool snapped off. Need to replace tool and inspect spindle for damage. Looks like they were cutting too deep in one pass for the strength of the tool"
"The DOC is too large and the feed too high for the slot such that the forces increase until tool breakage as the tool approaches the vice. It probably wasn't smart either to machine towards the vice as they have anyway. A typical approach to avoid this problem is to ramp into the slot."
"All-around operator error. Looks to be too high a depth of cut at too high a feedrate. Also looks like the move at the end put too high a stress on the tool. Operator should have retracted the tool before making that move if he/she wanted to keep that depth of cut."

"Too large of an engagement at tool high of a feed." 


\section{Interactive Case Study}

"The cutting tool snapped off. Need to replace tool and inspect spindle for damage. Looks like they were cutting too deep in one pass for the strength of the tool"
"All-around operator error. Looks to be too high a depth of cut at too high a feedrate. Also looks like the move at the end put too high a stress on the tool. Operator should have retracted the tool before

\section{Tool is broken}

e if he/she wanted to depth of cut."

"The DOC is too large and the feed too high for the slot such that the forces increase until tool breakage as the tool approaches the vice. It probably wasn't smart either to machine towards the vice as they have anyway. A typical approach to avoid this problem is to ramp into the slot."
"Too large of an engagement at tool high of a feed." 


\section{Interactive Case Study}

"The cutting tool snapped off. Need to replace tool and inspect spindle for damage. Looks like they were cutting too deep in one pass for the strength of the tool"
"All-around operator error. Looks to be too high a depth of cut at too high a feedrate. Also looks like the move at the end put too high a stress on the tool. Operator should have retracted the tool before

\section{Depth of cut too large}

e if he/she wanted to

"The DOC is too large and the feed too high for the slot such that the forces increase until tool breakage as the tool approaches the vice. It probably wasn't smart either to machine towards the vice as they have anyway. A typical approach to avoid this problem is to ramp into the slot."

"Too large of an engagement at tool high of a feed." 


\section{Interactive Case Study}

"The cutting tool snapped off. Need to replace tool and inspect spindle for damage. Looks like they were cutting too deep in one pass for the strength of the tool"
"All-around operator error. Looks to be too high a depth of cut at too high a feedrate. Also looks like the move at the end put too high a stress on the tool. Operator should have retracted the tool before

\section{Feed rate too high}

e if he/she wanted to depth of cut."

"The DOC is too large and the feed too high for the slot such that the forces increase until tool breakage as the tool approaches the vice. It probably wasn't smart either to machine towards the vice as they have anyway. A typical approach to avoid this problem is to ramp into the slot."
"Too large of an engagement at tool high of a feed." 


\section{Interactive Case Study}

"The cutting tool snapped off. Need to replace tool and inspect spindle for damage. Looks like they were cutting too deep in one pass for the strength of the tool"
"All-around operator error. Looks to be too high a depth of cut at too high a feedrate. Also looks like the move at the end put too high a stress on the tool. Operator should have retracted the tool before

\section{Bad process plan}

ve if he/she wanted to

"The DOC is too large and the feed too high for the slot such that the forces increase until tool breakage as the tool approaches the vice. It probably wasn't smart either to machine towards the vice as they have anyway. A typical approach to avoid this problem is to ramp into the slot."
"Too large of an engagement at tool high of a feed." 


\section{Interactive Case Study}

"The cutting tool snapped off. Need to replace tool and inspect spindle for damage. Looks like they were cutting too deep in one pass for the strength of the tool"
"All-around operator error. Looks to be too high a depth of cut at too high a feedrate. Also looks like the move at the end put too high a stress on the tool. Operator should have retracted the tool before

\section{Operator error}

e if he/she wanted to depth of cut."

"The DOC is too large and the feed too high for the slot such that the forces increase until tool breakage as the tool approaches the vice. It probably wasn't smart either to machine towards the vice as they have anyway. A typical approach to avoid this problem is to ramp into the slot."
"Too large of an engagement at tool high of a feed." 


\section{Maintenance Work Order Data}

Raw Data

\begin{tabular}{|c|c|c|c|c|c|c|c|c|}
\hline \multicolumn{2}{|l|}{ Effect } & $\begin{array}{l}\text { Average of } \\
\text { Time to } \\
\text { Complete }\end{array}$ & Number of & Total Time to & Effect & $\begin{array}{c}\text { Average of } \\
\text { Time to } \\
\text { Complete } \\
\text { (hrs) }\end{array}$ & $\begin{array}{l}\text { Number of } \\
\text { Instances }\end{array}$ & $\begin{array}{l}\text { Total Time to } \\
\text { Complete (hrs) }\end{array}$ \\
\hline \multicolumn{2}{|c|}{ Accumulator check requested } & 1.4590 & 14 & 16.05 & Hydraulic Leak & 40.8775 & 39 & 817.55 \\
\hline \multicolumn{2}{|l|}{ Vogel lube faults } & 1.5875 & 7 & 6.35 & Accumulator check requested & 1.690 & 26 & 35.5 \\
\hline \multicolumn{2}{|l|}{ Base cleaning requested } & 13.5 .75 & 4 & 27.15 & Coolant Leak & 122.47 & 17 & 1347.2 \\
\hline \multicolumn{2}{|l|}{ Table index $\mathrm{O} / \mathrm{T}$ faults } & $2.7 \backslash$ & 3 & 2.7 & Bearings check & 16.835 & 16 & 168.35 \\
\hline \multicolumn{2}{|c|}{ lemca will not load in Auto } & $313.2 \backslash$ & 3 & 939.6 & Chip conveyor INOP & 5.8 & 15 & 63.8 \\
\hline \multicolumn{2}{|l|}{ Chip conveyor INOP } & 1.075 & 3 & 2.15 & Broken screw & 3.8722 & 14 & 34.85 \\
\hline \multicolumn{2}{|l|}{ Chip conveyor jammed } & 3.725 & 13 & 7.45 & Table index faults & 24.08 & 13 & 120.4 \\
\hline \multicolumn{2}{|l|}{ St\#2 drill detector INOP } & 0.15 & 12 & 0.15 & Brush unit stuck forward & 4.744 & 10 & $42.7 \quad$ \\
\hline \multicolumn{2}{|c|}{ Table drifting at $1 / 2$ table setting } & 47 & 2 & 94 & Vogel lube fault & 2.27 & 9 & $11.35 !$ \\
\hline \multicolumn{2}{|c|}{ Motor thermal overload fault -Hydraulic } & 24 & 21 & 24 & Coolant Pressure Low & 3.26 & 9 & 16.3 \\
\hline \multicolumn{2}{|c|}{ Machine will not run in Auto } & & $2 !$ & & Oil leak & 39.2375 & 8 & 156.95 \\
\hline \multicolumn{2}{|c|}{ Part not loading into collet } & & 2 & & Base cleaning requested & 13.575 & 4 & 27.15 \\
\hline \multicolumn{2}{|c|}{ St\#8 Hyd flange not repeating } & 0.15 & 2 & 0.15 & lemca will not load in Auto & 235.9 & 4 & 943,6 \\
\hline \multicolumn{2}{|l|}{ Power pack leak } & & 2 & 1 & Bearings noise & 79 & 4 & 79 \\
\hline \multirow[t]{4}{*}{$\begin{array}{l}\text { Table index } \mathrm{O} / \mathrm{T} \text { at } 1 / 2 \mathrm{t} \\
\text { Hydraulics }\end{array}$} & ble -Turning off & & 2 & & Inverter failing to return & 0.3 & 4 & 0.3 \\
\hline & & & & 1 & Total Time to Cor & iplete & (hrs) & l \\
\hline & & & ffect & & Raw & Clea & & 1 \\
\hline & Accumu & lator ch & eck req & lested & 16.05 & 35. & & \\
\hline
\end{tabular}

Clean Data 
Hydraulic leak at cutoff unit

Hyd leak at St\#2 chip breaker valve Hyd leak reported

Hydraulic leak at bar loader -Rubber seal on vacuum

HP Hydraulic line ruptured

Multiple leaks at lemca - 25 Gallons in 48 hours

Hydraulic return line leak

Hyd leak from behind collet \#6

Hydraulic leak turret 2

Hydraulic leak actuator or horseshoe

Hydraulic leak at chip breaker valve (? Valve station) Hydraulic leaks -from collets??

Leak at High Pressure pump

Hyd leak St\#2 valve

St\#6 valve leaking hydraulic Hydraulic leak

Hyd leak at locking pin assy

lemca hydraulic pump leaking -Full tank per day

Hydraulic leak on Side A

Hydraulic leak from power pack

St\#8 valve leaking Hyd fluid

Hyd leaks - C/O unit, St\#11 Valve, Collet \#10 (Internal)

Hydr pump? / Power pack leak / CNCs shuddering Hydraulic leak at inverter st $\# 8$ Hyraulic leak at St\#4

Hyd leaks at valve below \#7 / Lid leaks at loader

St\#8 valve spraying hydraulic fluid

Hyd leak at lemca pumps tank

Hyd leak from dressing unit

Hydraulic leak at Cutoff valve

Hydraulic leak at power pack -per PM tix

Hydraulic leak found by Doug -3.1 quil

Hydraulic Leak reported -One tank per day

Hydraulics leaking from dressing unt

$$
\text { Major hydraulic leak }
$$

Major Hydraulic leak at rotator -Rotator rack is broken

Hydraulic oil getting into Vogel waste ol

Clean Data

Hydraulic Leak 
Hydraulic return line leak Hyd leak from behind collet \#6

Hydraulic leak turret 2

Hydraulic leak actuator or horseshoe

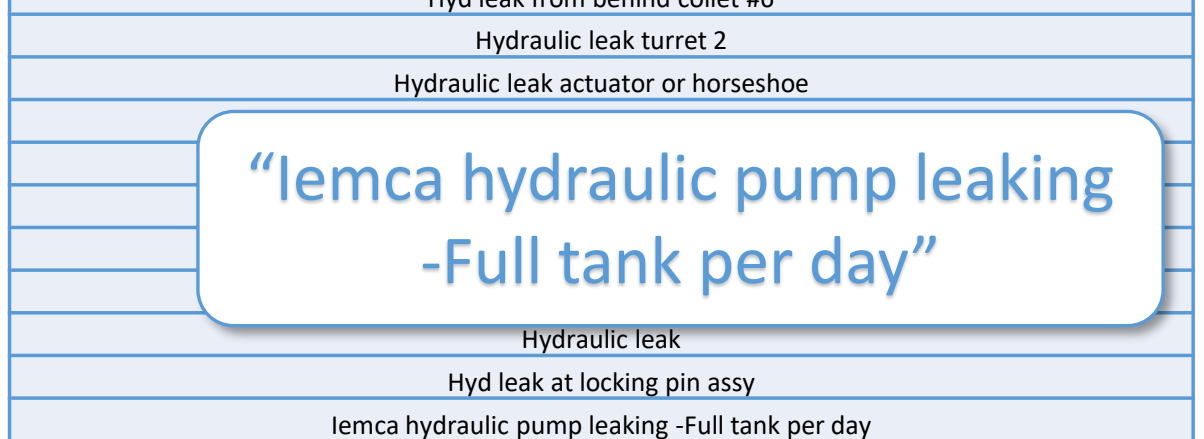

lemca hydraulic pump leaking -Full tank per day Hydraulic leak on Side A

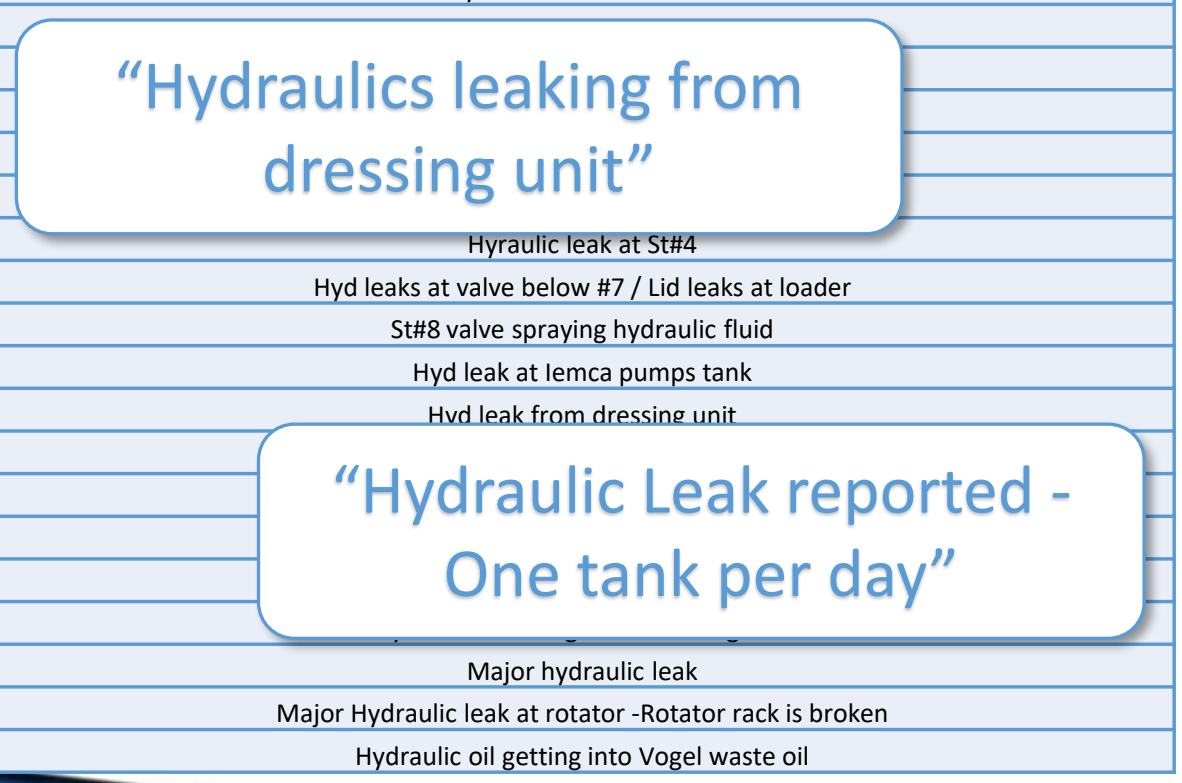

Clean Data

Hydraulic Leak 


\section{Transforming the MWO Data}

\section{Initial Annotation Attempt}

\section{Method}

- Find useful representations for common problems

- Unify terminology

- Preliminary Cause/Effects/Treatments

\begin{tabular}{l|ll} 
& Time Inv. & Result \\
\hline Initial annotation & 12 hours & 800 labels
\end{tabular}




\section{Transforming the MWO Data}

\section{Tagging as Annotation}

- "Tags" have many benefits for users:

- No Controlled Vocabulary

- Naturally reflect the user-base's communication

- Less ambiguous than strict classification $\rightarrow$ more usage

- Collections of tags on a domain form a "Folksonomy"

- Relationships are encoded via tag co-occurrence - like Bag of Words!

- Can be predicted via Multi-label classification

We sacrifice certainty about specific labels, and gain annotation quality \& ease-of-use 


\section{Transforming the MWO Data}

\author{
Description \\ Hydraulic Leak at cutoff unit
}

Item

Action

Problem

\section{$\underline{\text { Resolution }}$}

Missing fitting replaced

Action

$\underline{\text { Item }}$ 


\section{Transforming the MWO Data}

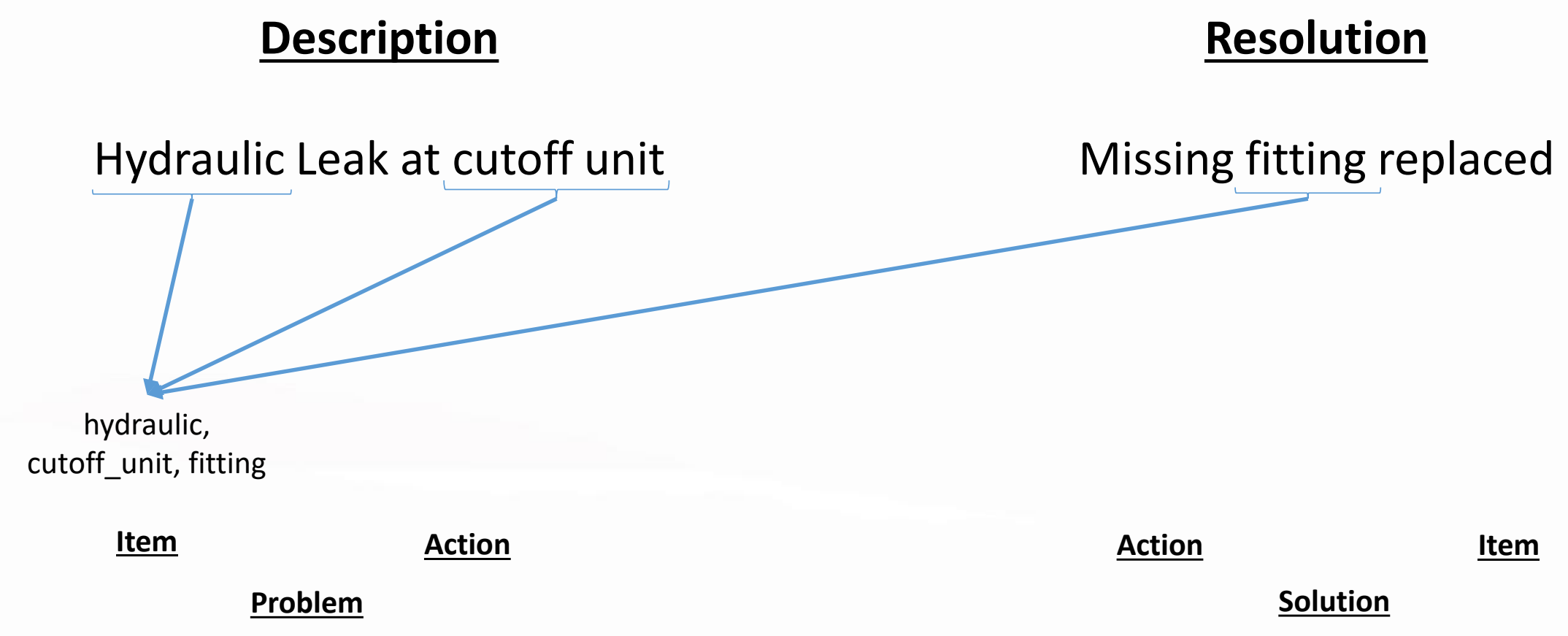




\section{Transforming the MWO Data}

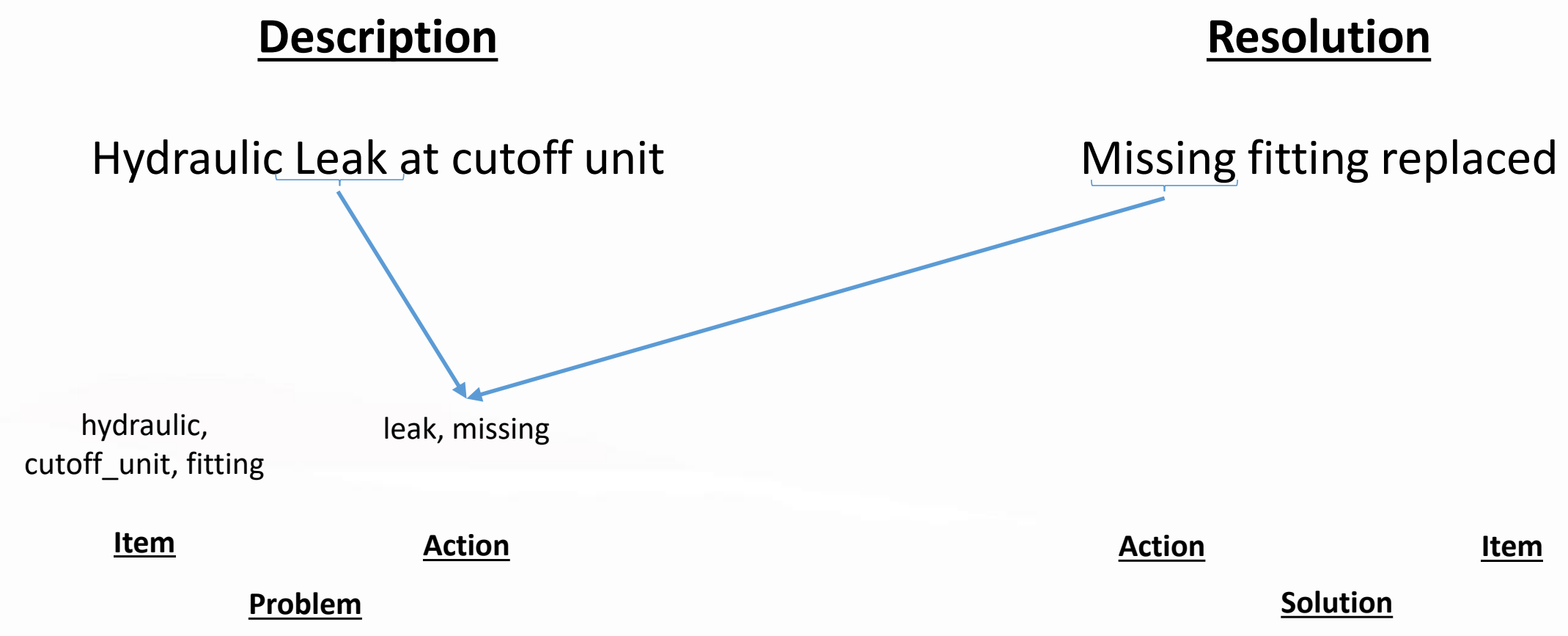




\section{Transforming the MWO Data}

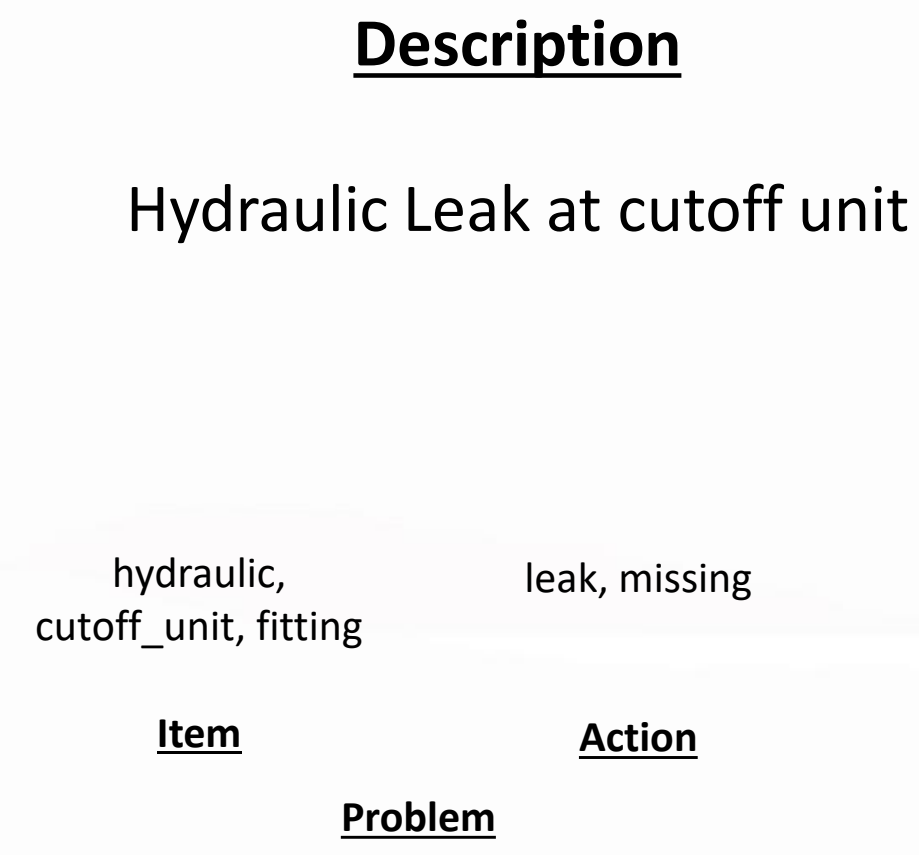

\section{$\underline{\text { Resolution }}$}

Missing fitting replaced

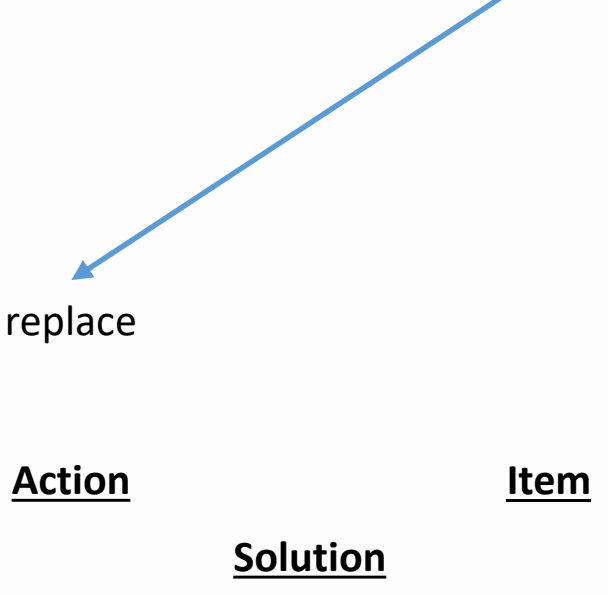




\section{Transforming the MWO Data}

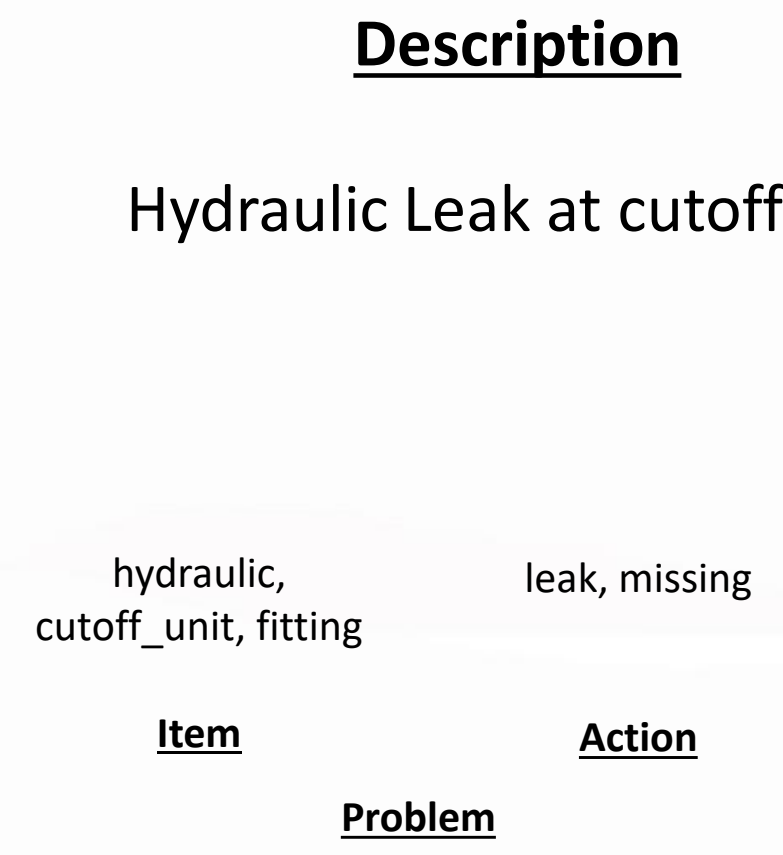

\section{$\underline{\text { Resolution }}$}

Missing fitting replaced

replace

Action Solution 


\section{Transforming the MWO Data}

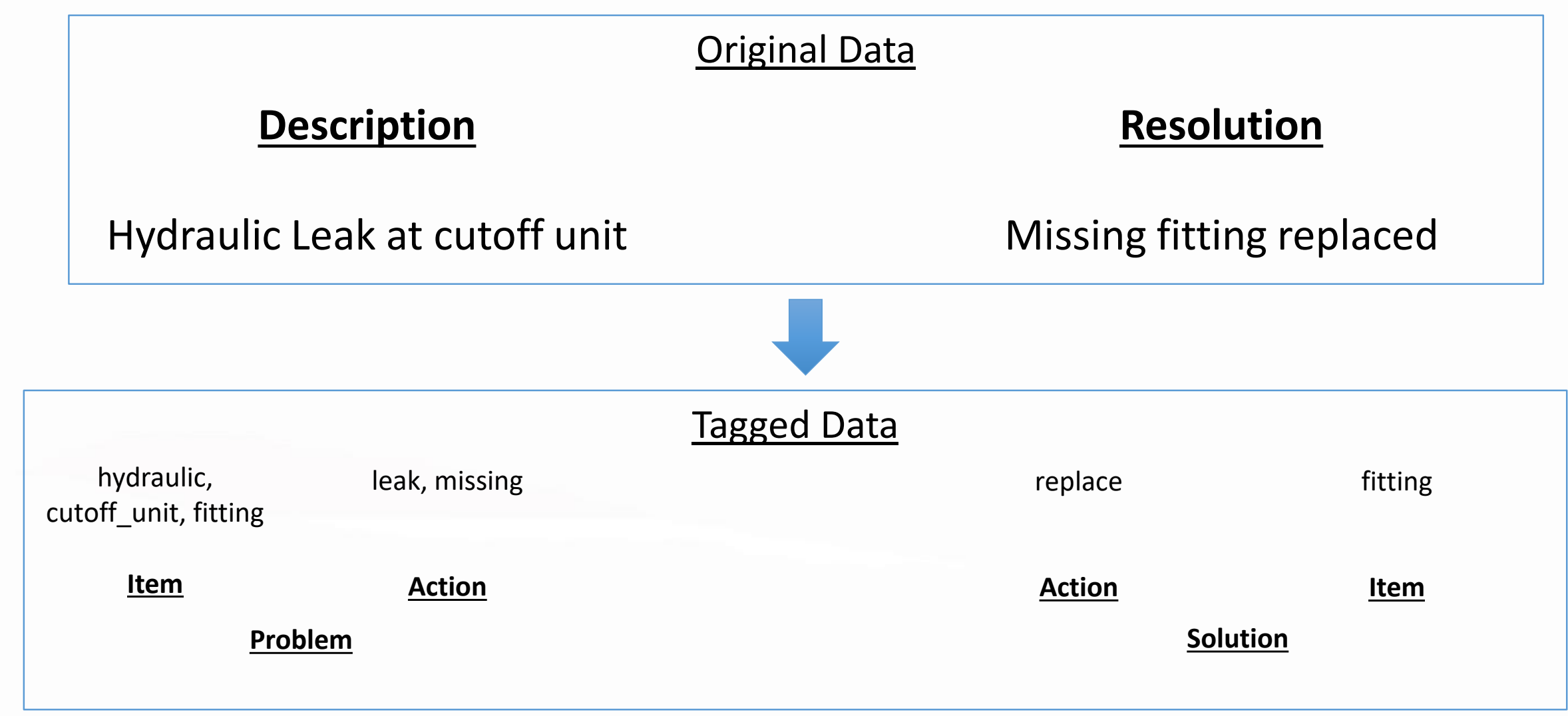




\section{Transforming the MWO Data}

Issue

"Hydraulic Leak at cutoff unit; Missing fitting replaced"

\section{Problem}

cutoff_unit

hydraulic

fitting leak

missing

\section{Solution}

replace

\section{fitting}

\section{Old classification}

"Hydraulic Leak"/OR

"Cutoff unit Leak"/OR

"Missing Fitting"

"replace fitting" 


\section{Transforming the MWO Data}

\begin{tabular}{l|ll} 
& Time Inv. & Result \\
\hline Previous annotation & 12 hours & 800 labels \\
Tagging method & 12 hours & 1200 tagged
\end{tabular}




\section{Semi-Automated Tagging}

\section{Keyword-based Tagging Tool}

Ordered Vocabulary Annotation

\begin{tabular}{|c|c|c|c|}
\hline & token & type & alias \\
\hline \multirow{10}{*}{$\begin{array}{c}\text { More } \\
\text { Important }\end{array}$} & replace & $\mathrm{s}$ & \\
\hline & unit & 1 & \\
\hline & motor & 1 & \\
\hline & spindle & 1 & \\
\hline & leak & $\mathrm{P}$ & \\
\hline & valve & 1 & \\
\hline & replaced & s & replace \\
\hline & fault & $\mathrm{P}$ & \\
\hline & bar & 1 & \\
\hline & inop & $\mathrm{P}$ & inoperable \\
\hline
\end{tabular}

Keyword Extractor

Tags Extracted from Work-order "Hydraulic Leak at cutoff unit; Missing fitting replaced"

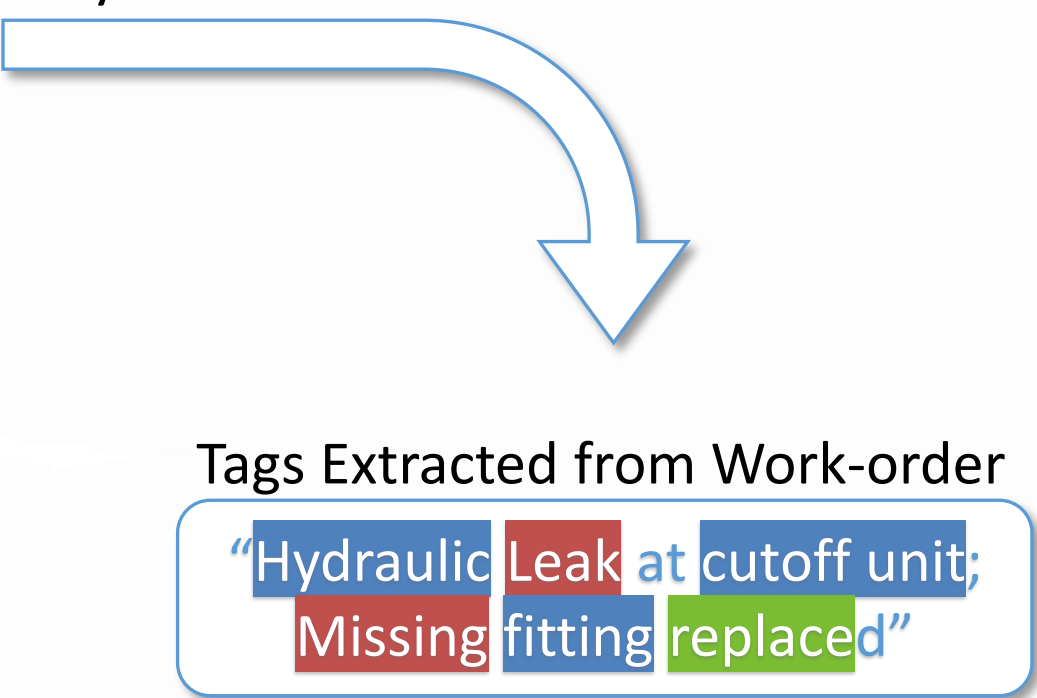




\section{Semi-Automated Tagging}

\begin{tabular}{l|ll} 
& Time Inv. & Result \\
\hline Previous annotation & 12 hours & 800 labels \\
Tagging method & 12 hours & 1200 tagged \\
& & \\
$\begin{array}{l}\text { Ordered Keyword } \\
\text { Tagger }\end{array}$ & 0.75 hours & $\begin{array}{l}3100 \text { tagged, } \\
99.7 \% \text { partials }\end{array}$
\end{tabular}




\section{Semi-Automated Tagging}

\begin{tabular}{|c|c|c|c|c|c|}
\hline \multicolumn{6}{|c|}{ 메 EATT Knowledge: TagTool } \\
\hline \multirow{2}{*}{\multicolumn{2}{|c|}{1 Gram Token }} & \multirow{2}{*}{\multicolumn{2}{|c|}{ N Gram Token }} & \multirow{2}{*}{$\begin{array}{l}\text { Report } \\
\text { Tag Annotation }\end{array}$} & \\
\hline & & & & & \\
\hline 1 & $\begin{array}{l}\text { tokens } \\
\text { replace }\end{array}$ & $\begin{array}{l}\text { NE } \\
\text { S }\end{array}$ & $\begin{array}{l}\text { alias } \\
\text { replace }\end{array}$ & notes & $\wedge$ \\
\hline 2 & bucket & I & bucket & & \\
\hline 3 & repair & S & repair & & \\
\hline 4 & grease & U & grease & & \\
\hline 5 & leak & $P$ & leak & & \\
\hline 6 & oil & U & oil & & \\
\hline 7 & engine & I & engine & & \\
\hline 8 & hose & I & hose & & \\
\hline 9 & broken & $P$ & broken & & \\
\hline 10 & tooth & 1 & tooth & & \\
\hline 11 & pump & I & pump & & \\
\hline 12 & lube & & & & $\checkmark$ \\
\hline
\end{tabular}

Similar Pattern Property Editor

$\checkmark$ lube Preferred Alias

$\square$ lubing lube

$\square$ lubricate

$\square$ louder $\bigcirc$ Item

$\square$ leg $\bigcirc$ Problem

$\square$ led

$\square$ lhe

$\square$ leak

$\square$ line

Solution

$\square$ left

Ambiguous (Unknown)

Stop-word

(2) not yet classified

Notes 


\section{Case Studies: Machine Performance}
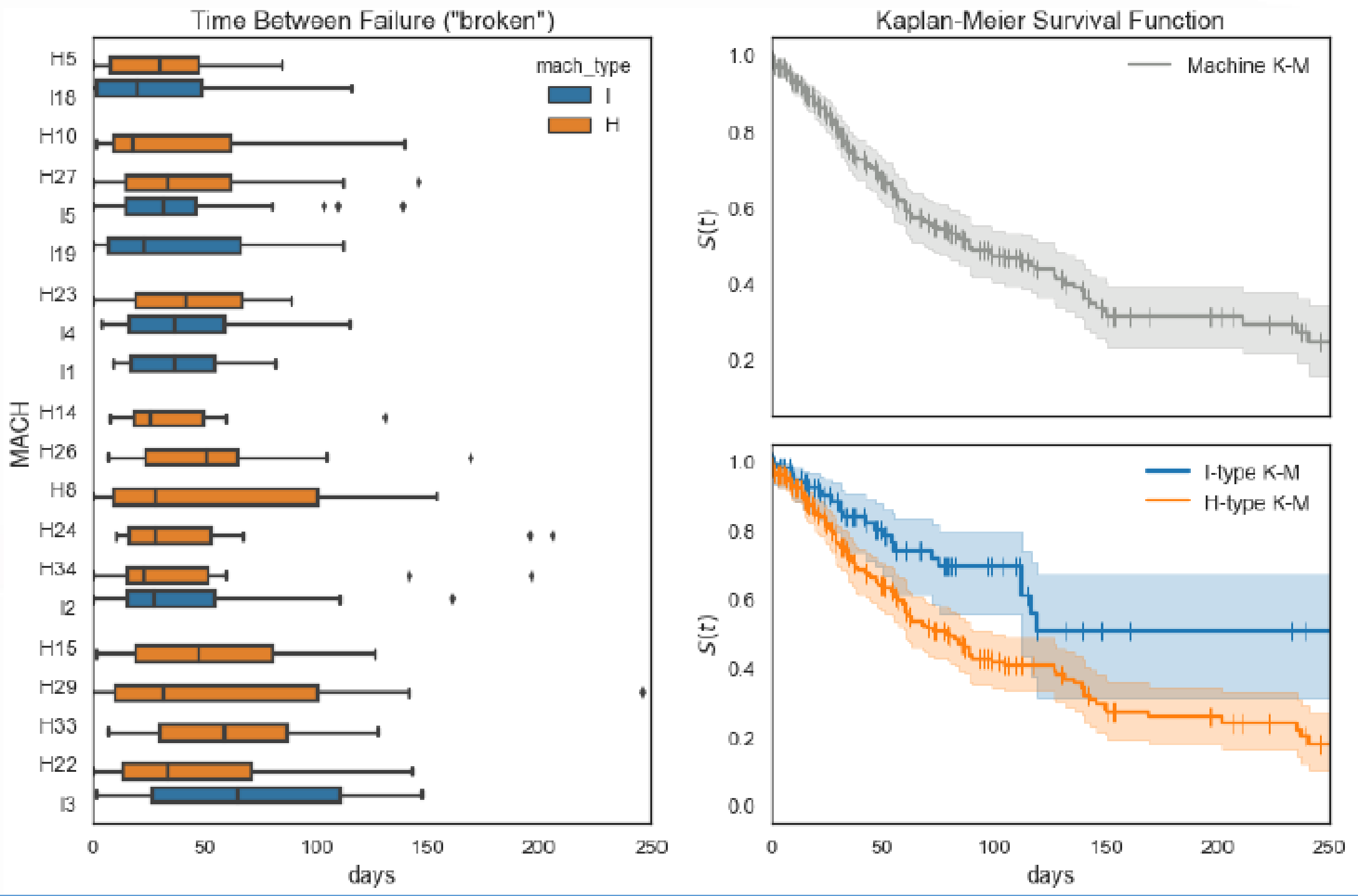


\section{Case Studies: Machine Performance}

Top Tag occurences, by Machine
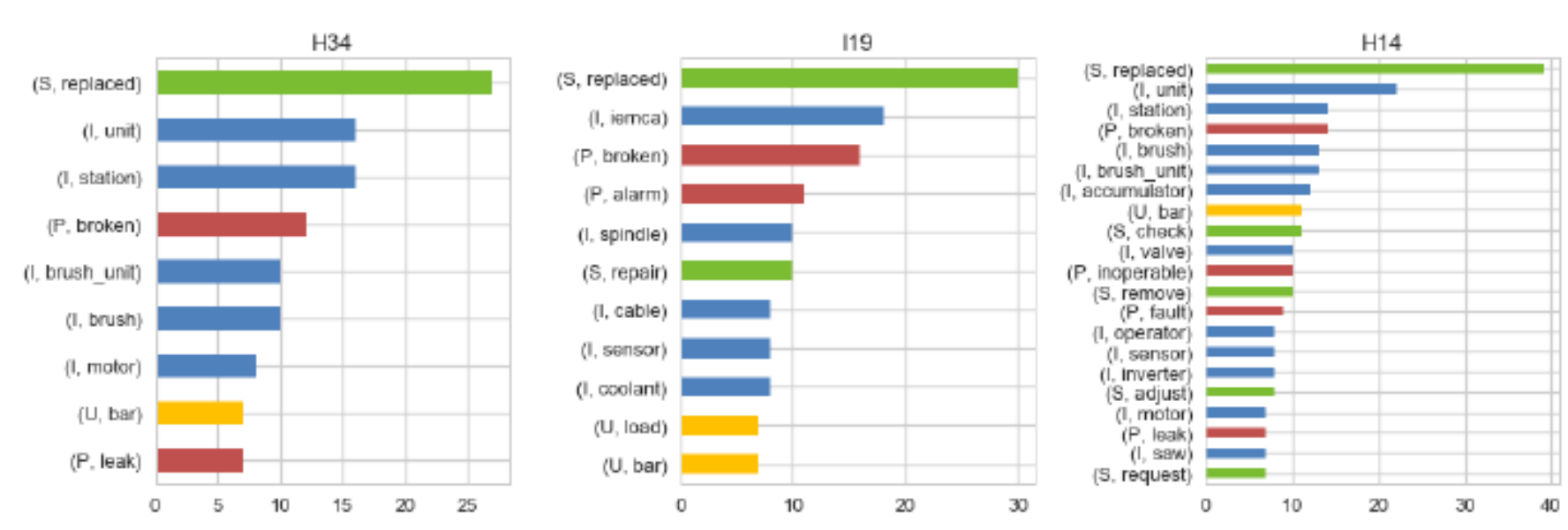

- H34 issues with motor, brush_unit

- I19 a larms and/or sensors, potentially coolant-related

- H14 wide array of issues, including operat or (!?)

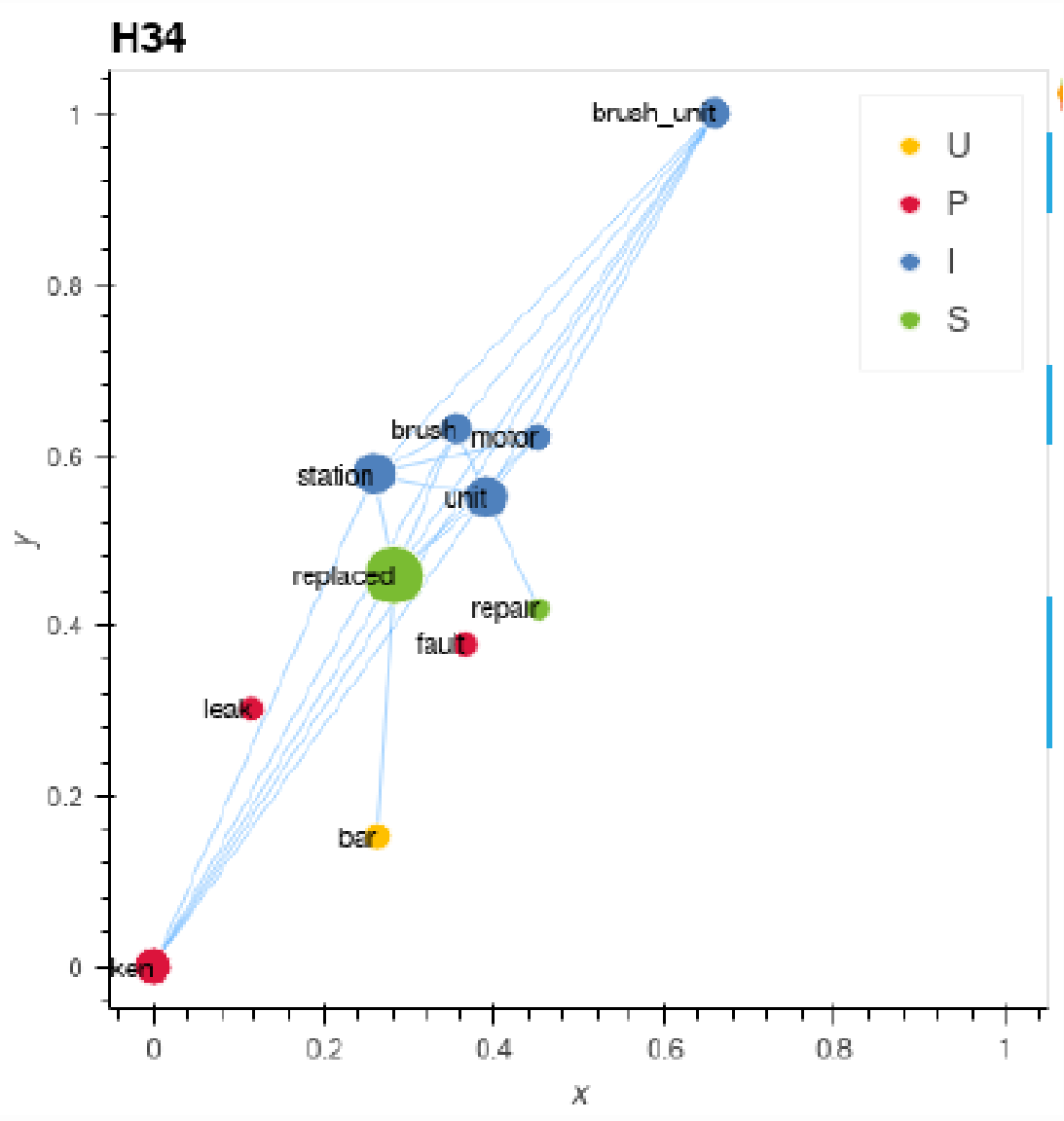




\section{Case Studies: Technician Performance}
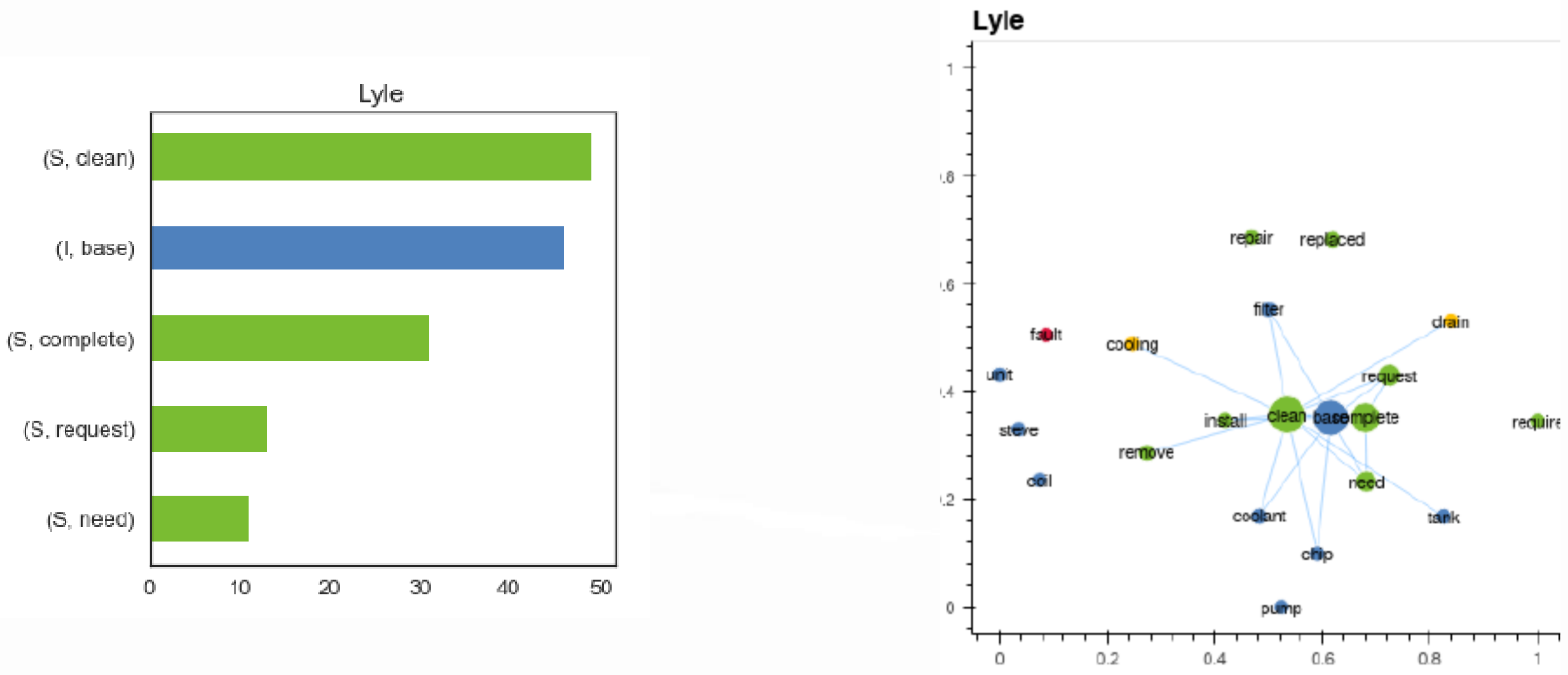


\section{Case Studies: HVAC Hotspot Identification}
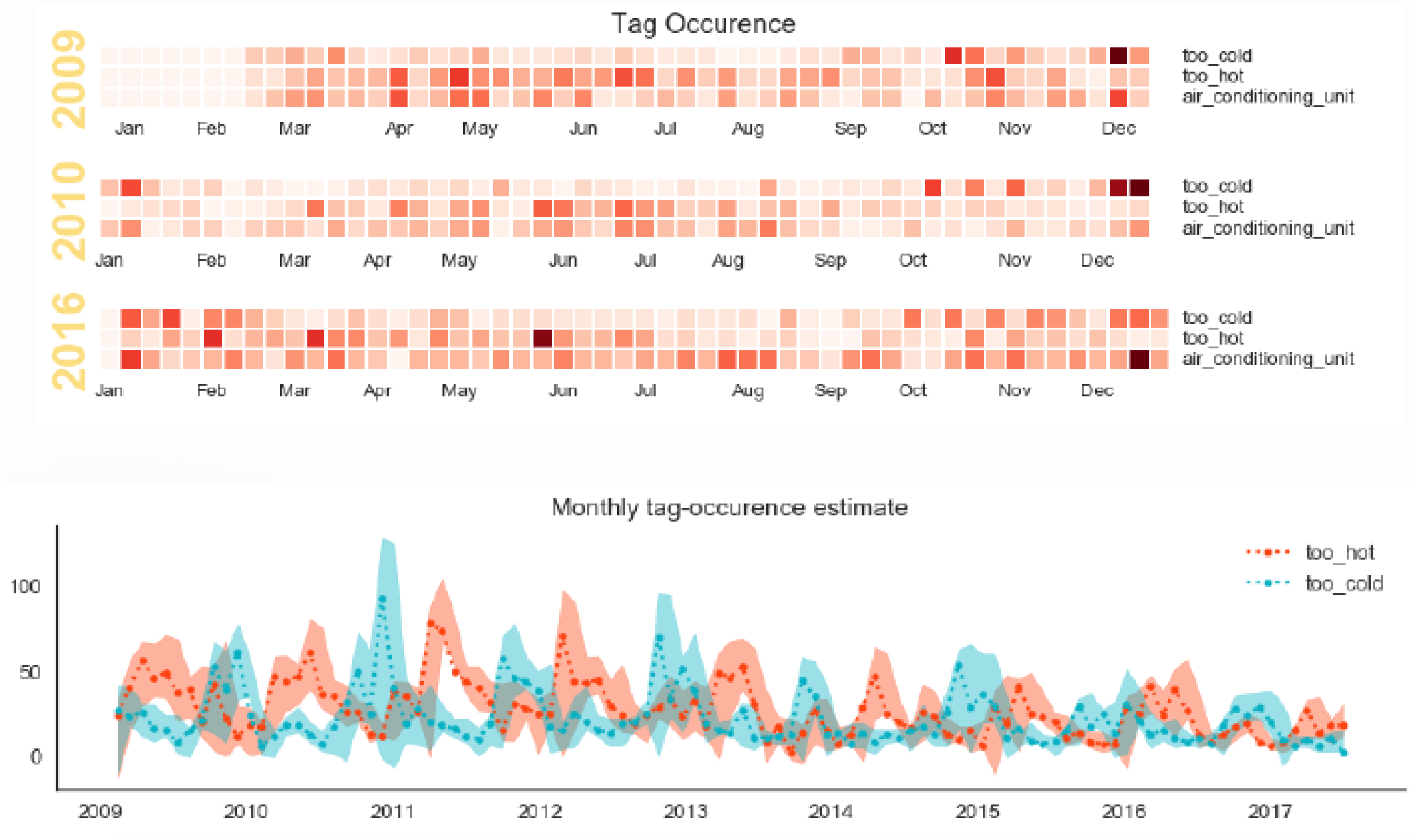


\section{Case Studies: HVAC Hotspot Identification}
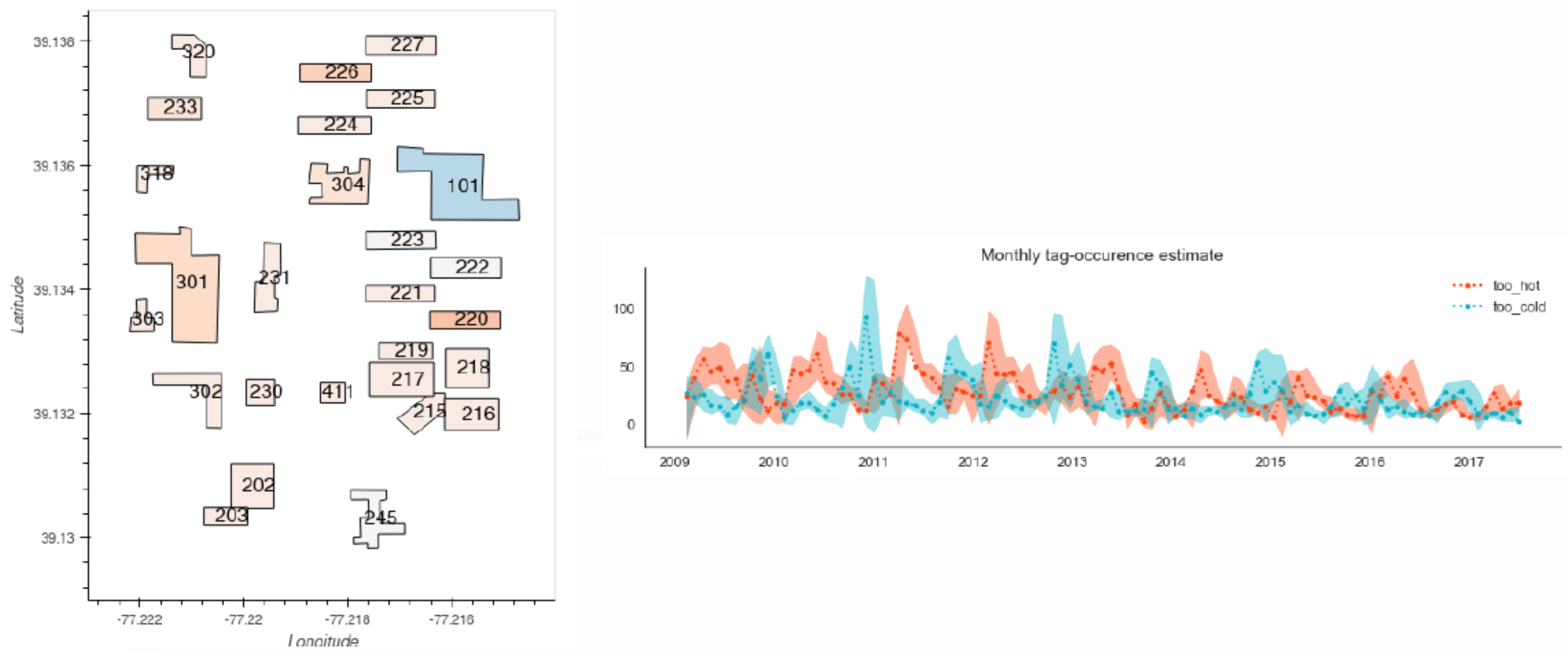


\section{Comparing Manufacturers}

Words described in

MWOs

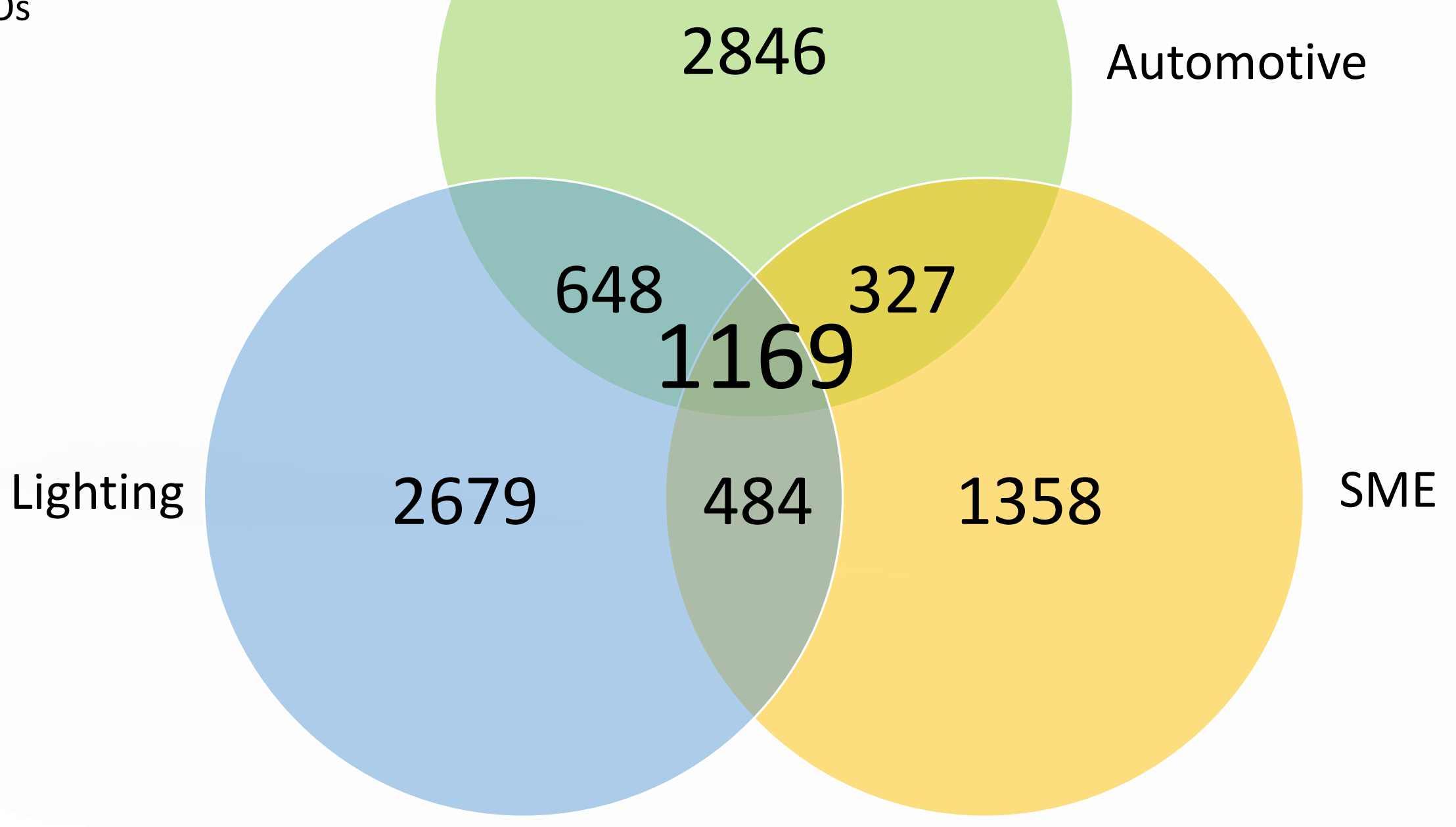




\section{Future Work}

- Tagging UI refinement and industry user studies

- Visualization UI

- Explore alternative visualizations

- Incorporate other data sources

- More use cases

- Automated hierarchy generation and V\&V

- Develop standard guidelines through ASME PHM Subcommittee 


\title{
Thank you!!
}

\author{
Michael Brundage \\ michael.brundage@nist.gov
}

Thurston Sexton

Thurston.sexton@nist.gov 


\section{Industry Forum}

NIST Research on Monitoring, Diagnostics, and Prognostics for Manufacturing Workcells

Brian A. Weiss, Project Leader Prognostics, Health Management, and Control

Intelligent Systems Division Engineering Laboratory National Institute of Standards and Technology

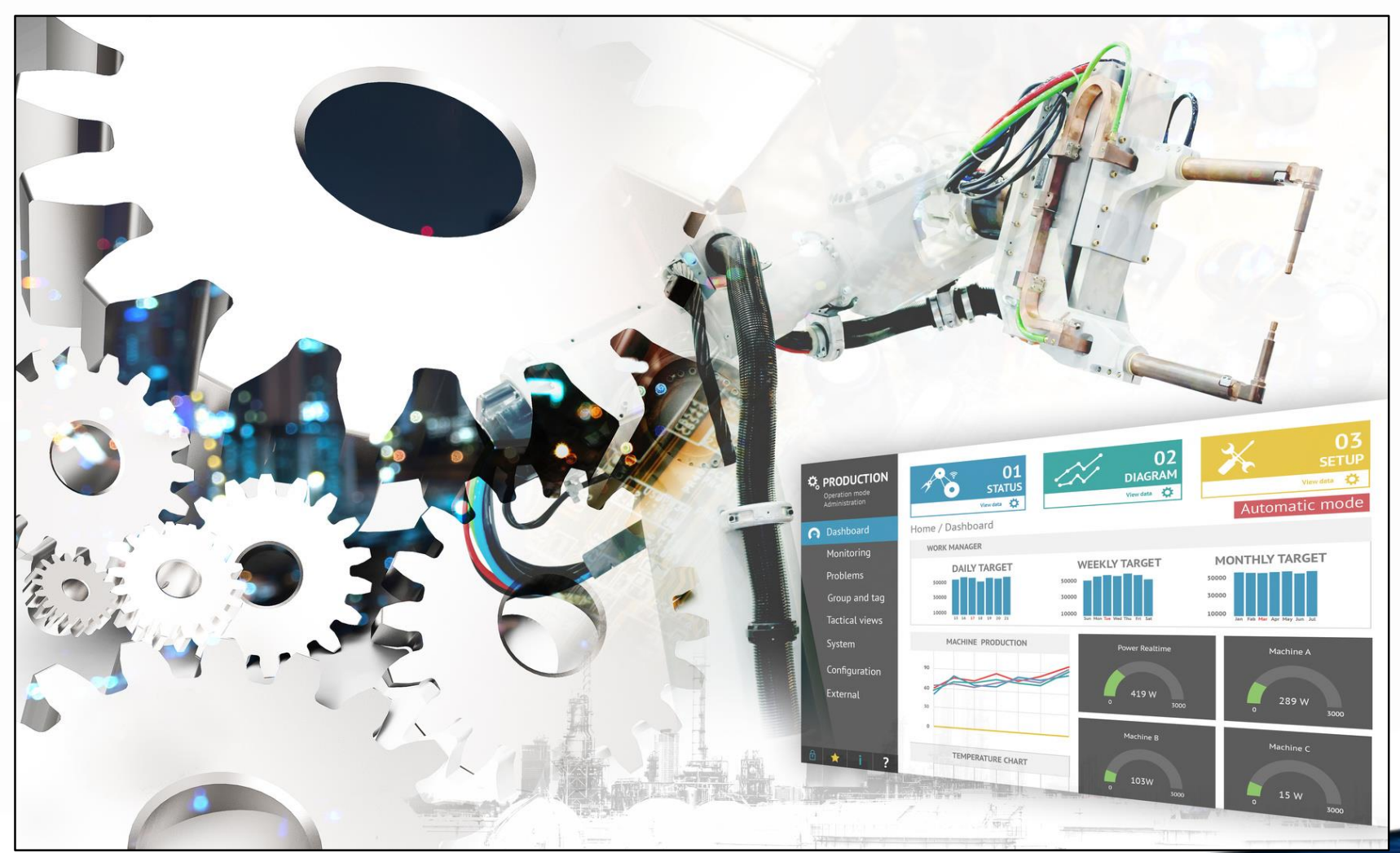




\section{Disclaimer}

- Identification of commercial systems does not imply recommendation or endorsement by NIST

- Identified commercial systems are not necessarily the best available for the purpose 


\section{The Most Critical Piece of the Project...}
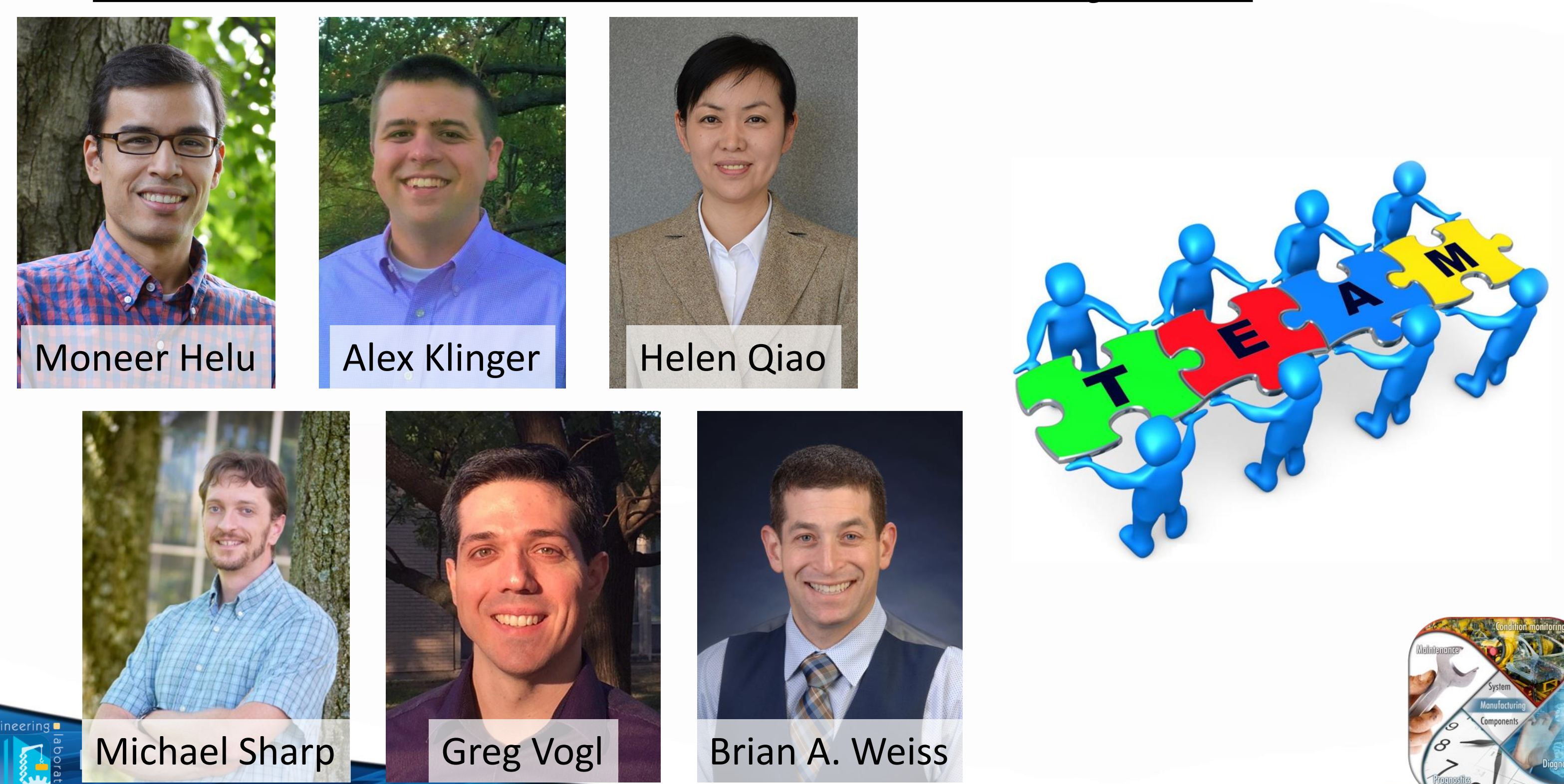


\section{Research Objective and Deliverables}

The PHMC project will deliver Measurement Science Products for robust sensing, diagnostics, prognostics, and control that enable manufacturers to respond to planned and un-planned

ASME

ilit'Connect Instruite

Standards and Guidelines

○

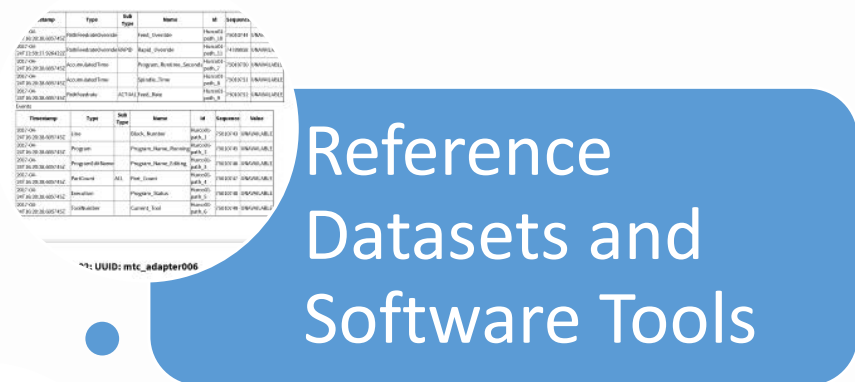
performance changes thereby enhancing the efficiency of smart manufacturing systems.
Test Methods and

Performance Metrics 


\section{How do we know this is Important?}

Measurement Science Roadmap

for Prognostics and Health

Management for Smart

Manufacturing Systems

- Measurement Science Roadmapping Workshop

- Manufacturing Standards Requirements Gathering Workshop

- Collaborator studies with university and industry partners

- Interactions with various technical organizations

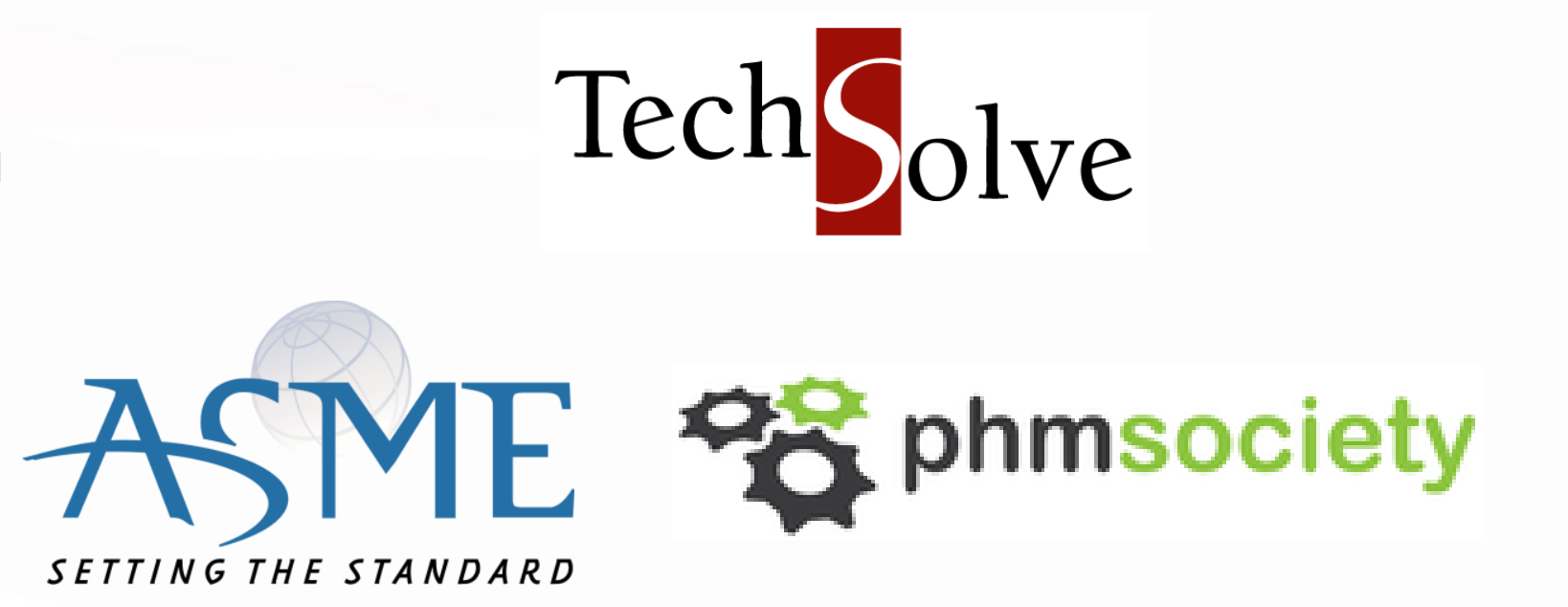

university OF

Cincinnati

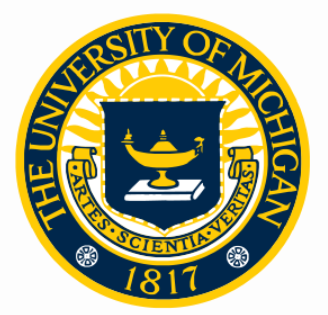

$$
\text { Tech Solve }
$$

울 phmsociety
NIST Advanced Manufacturing Series 100-13

Summary Report on a Workshop on Advanced Monitoring, Diagnostics, and Prognostics for Manufacturing Operations

ASAE 


\section{$\underline{\text { Research Levels }}$}

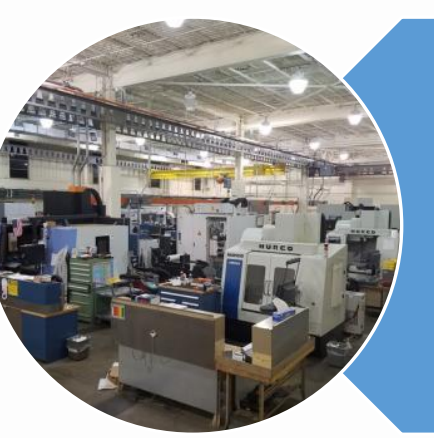

Manufacturing Process and Equipment Monitoring

- System-Level Research

- Smart Manufacturing Systems Testbed

Health and Control Management for Robot Workcells

- Work Cell-Level Research

- PHM for Robot Systems Lab/Testbed

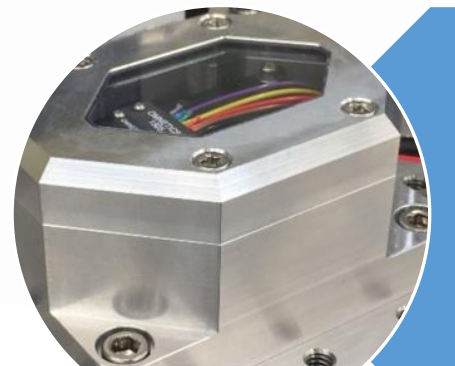

Machine Tool Linear Axes Diagnostics and Prognostics

- Component-Level Research

- Linear Axis Test bed \& 'Shops' Machine Tools 


\section{Manufacturing Workcells}

Robot Arm

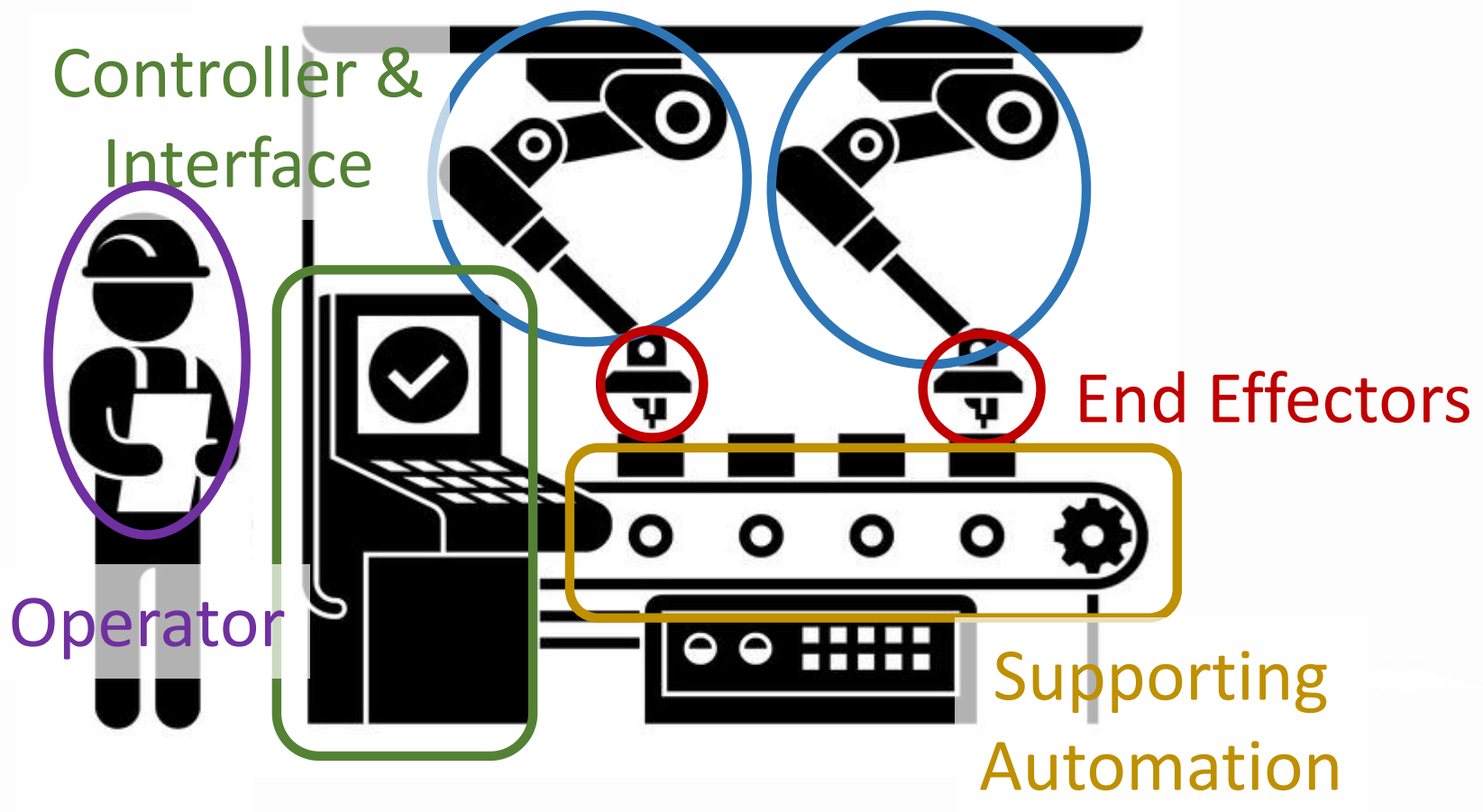

Potential Fault/Failure Sources

- Human

- Control/Software

- Mechanical

- Electrical

- Environmental 


\section{Robotic Workcell}

- Goal: Develop the necessary measurement science to enable the V\&V of monitoring, diagnostic, and prognostic technologies within a manufacturing robot work cell

- Impact: Increase equipment and process health intelligence through advanced monitoring, diagnostic, prognostic, and control strategies to optimize planned maintenance and minimize unplanned maintenance of manufacturing workcells

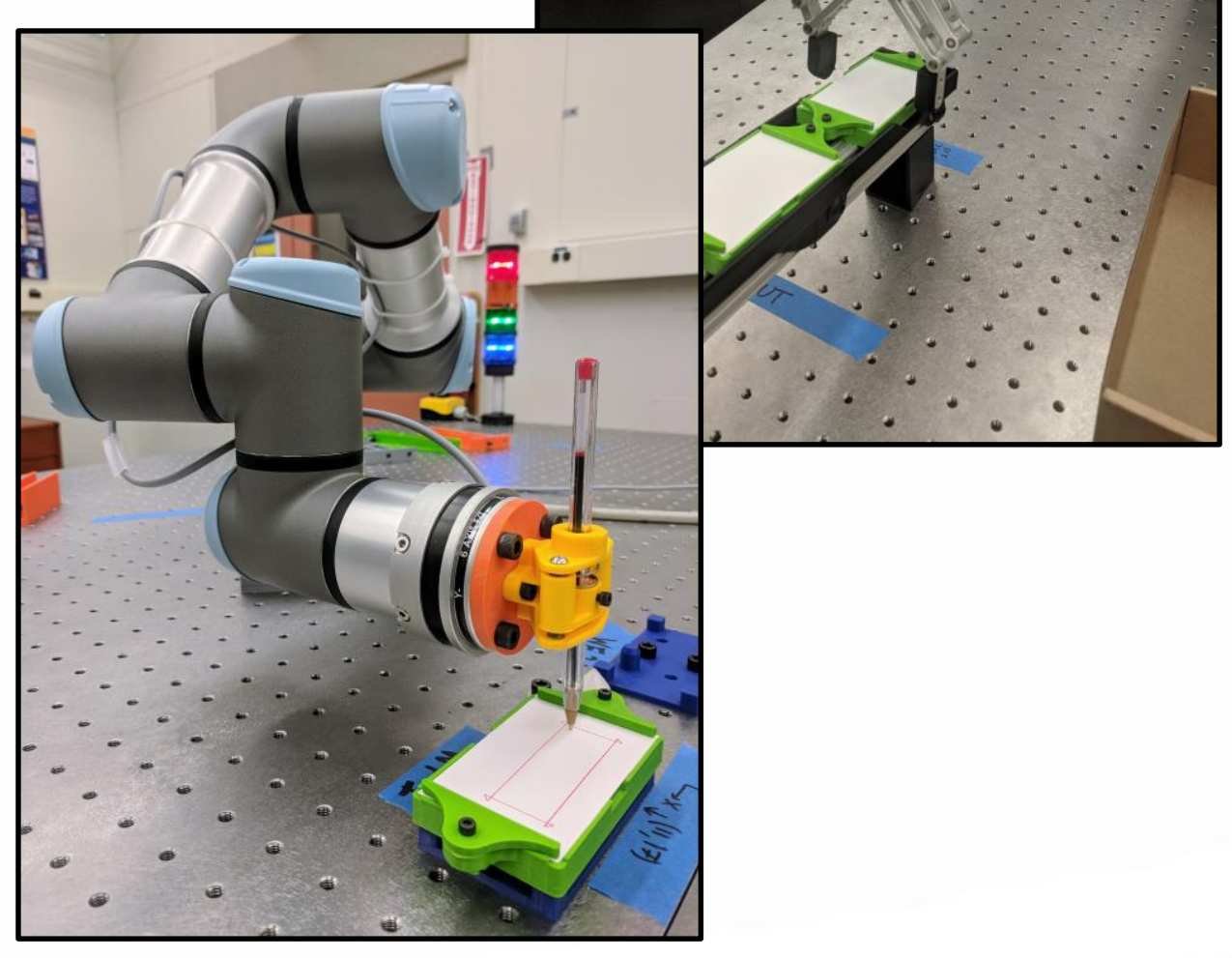




\section{Robotic Workcell - Use Case}

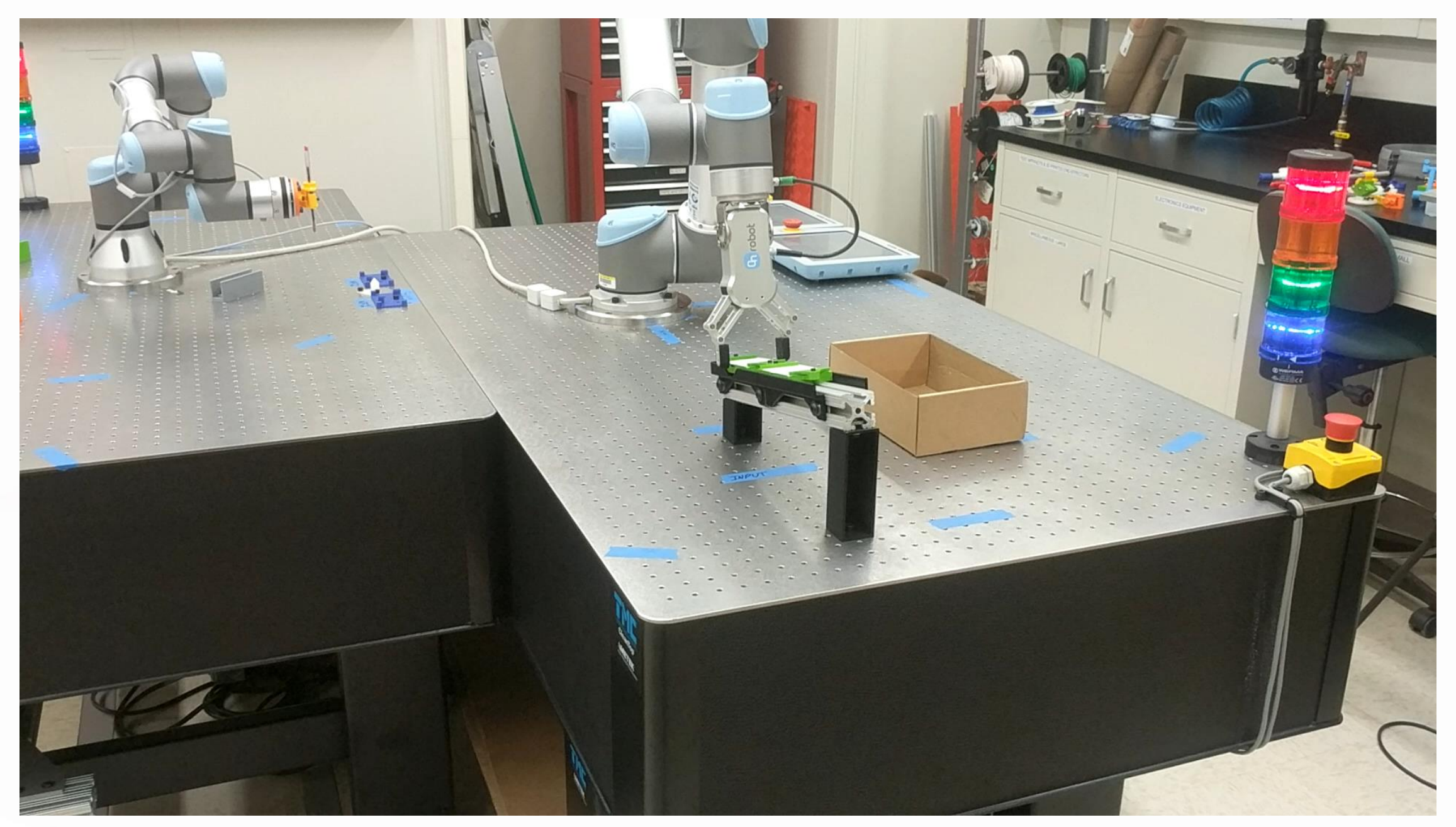

1)

Health Management, \& Control 


\section{Identifying Process Repeatability Degradation via Position}

Goal: To develop a low cost in situ method to identify source of process repeatability degradation within industrial robot enabled workcells with minimal process disruption.

Method: Workcells self-inspect at select points along kinematic chains to provide insight on if repeatability is degrading and where the degradation is occurring.

\section{Challenges:}

1. Identifying the points that should be measured / monitored along the kinematic chain

2. Choosing / developing sensing technology

User Defined

World (Set During Measurement)

Frame

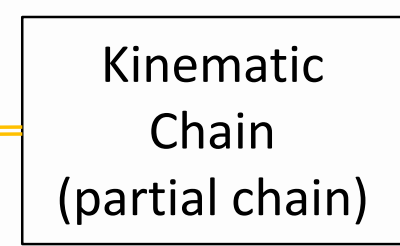

User Defined (Rigid)

Measurement

Point 


\section{Selection of Measurement Points - Material Handling Use Case}

$\longrightarrow \leftarrow$ Measurement Point

- Measurement Points chosen at specific locations along the kinematic chain to enhance information gained during inspection.

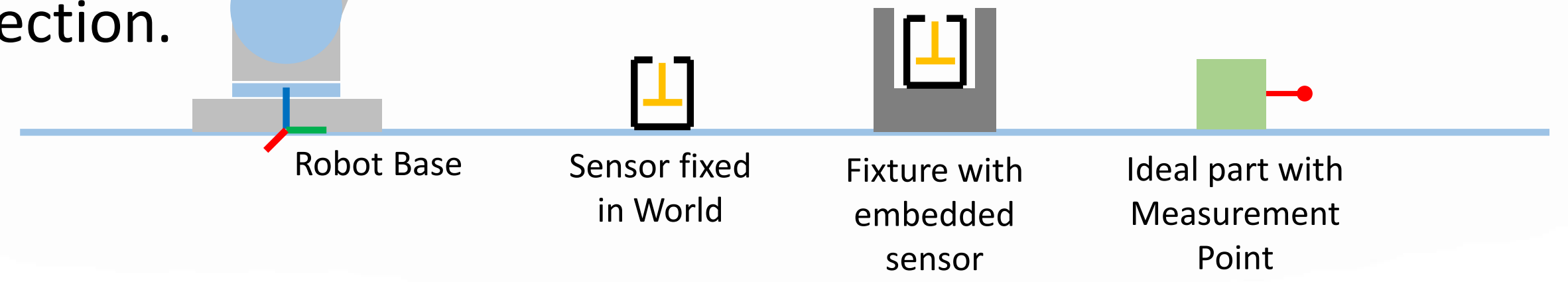

$\longrightarrow \longleftarrow$ Robot Tool Flange

$\longrightarrow \leftarrow$ Gripper Body

$\multimap \longleftarrow$ Gripper Finger

sensor 


\section{Work Cell Level Research - Quick Health Assessment Methodology}

- Research Objective: Develop a quick health assessment methodology to provide manufacturers with robot health intelligence to enhance maintenance and control decisions

- Key Output to Date:

- Advance sensing - 7-D measurement system

- Innovative target - smart target

- Algorithms and test method for quick robot position and orientation accuracy assessment

\section{- Impact:}

- Reference test methods will educate and guide manufacturers in deploying PHM to quickly assess robot health promoting greater employment of predictive maintenance strategies (e.g. robot system calibration, joint and gear box replacement etc.) that will increase efficiency and productivity while decreasing downtime.

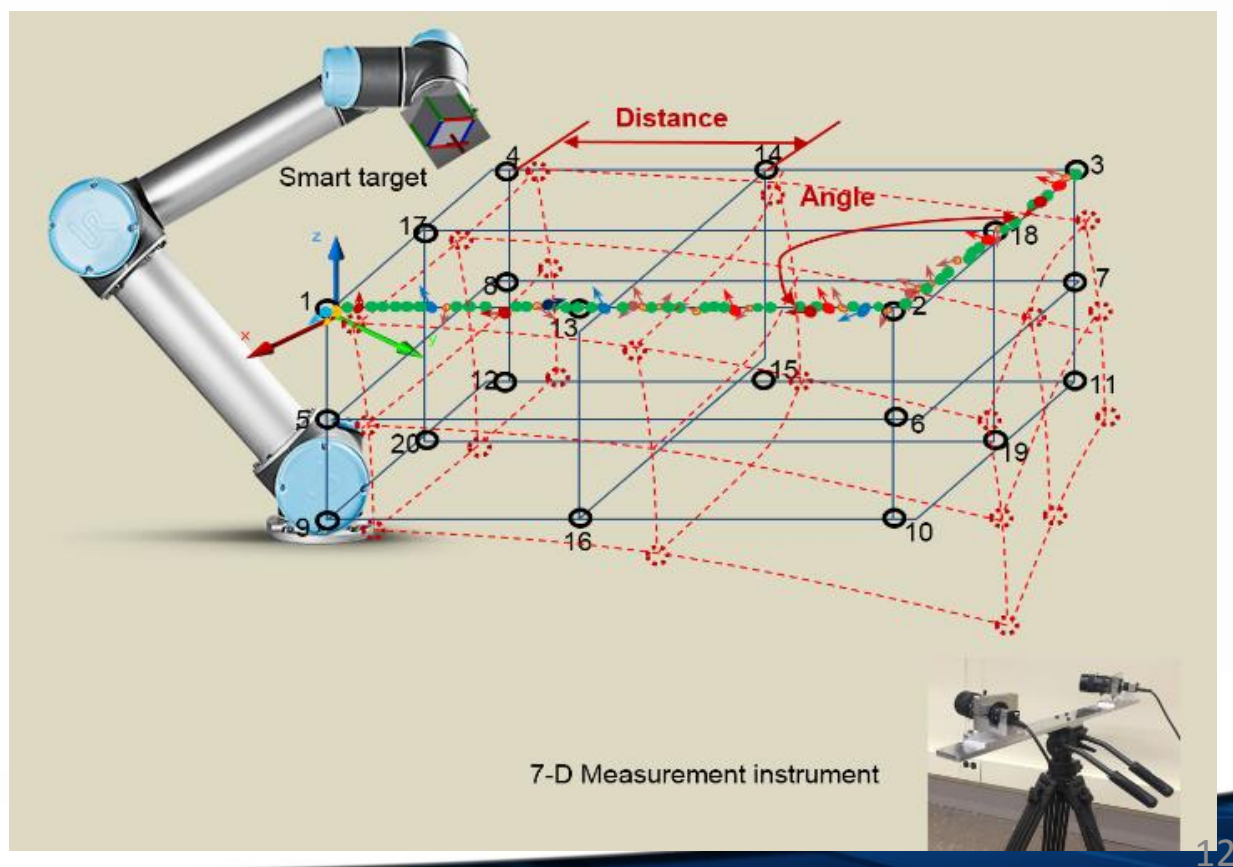




\section{Test Method Development and Reference Data Collection}

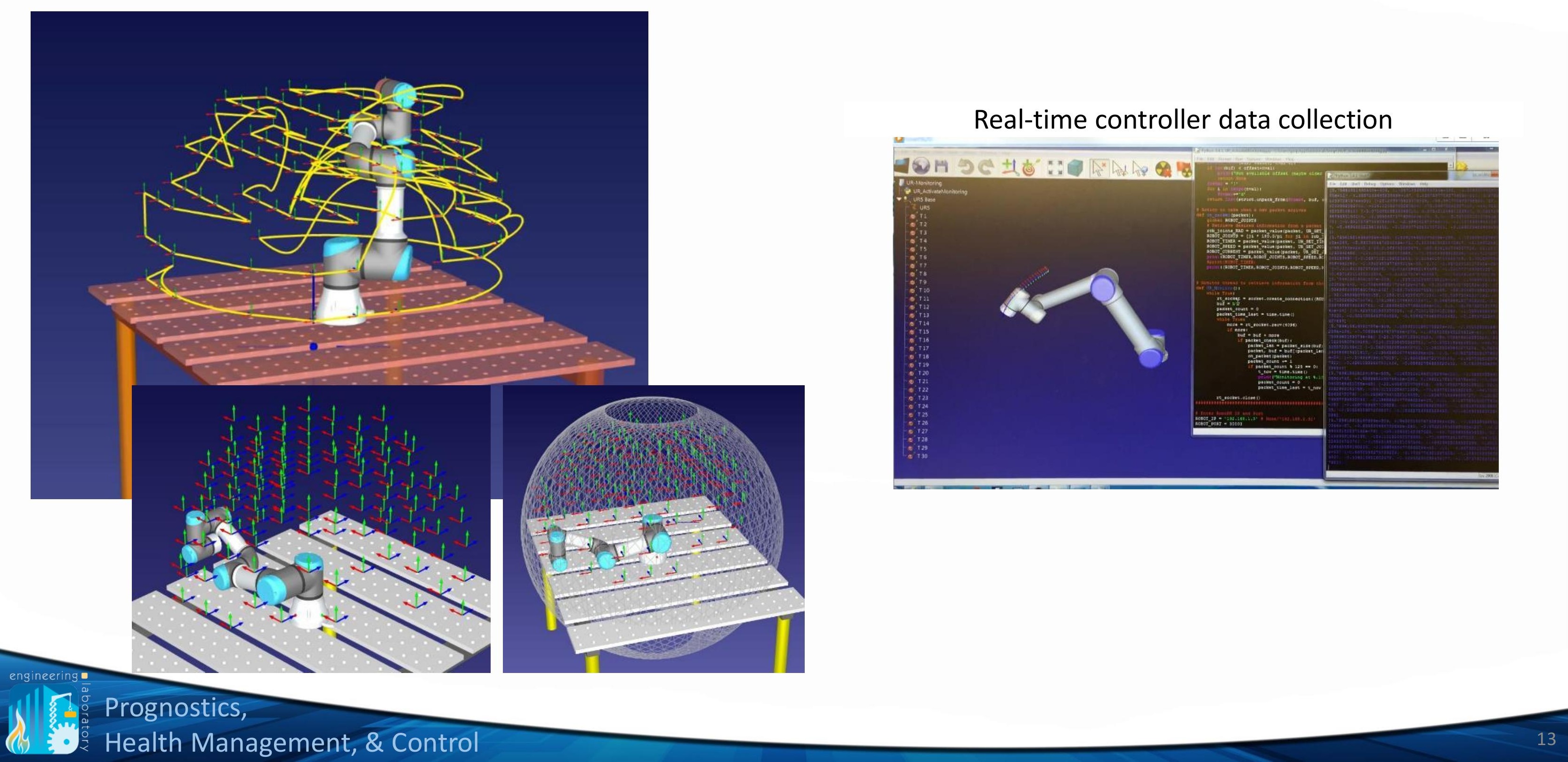




\section{$\underline{\text { Robot - Reference Data Sets }}$}
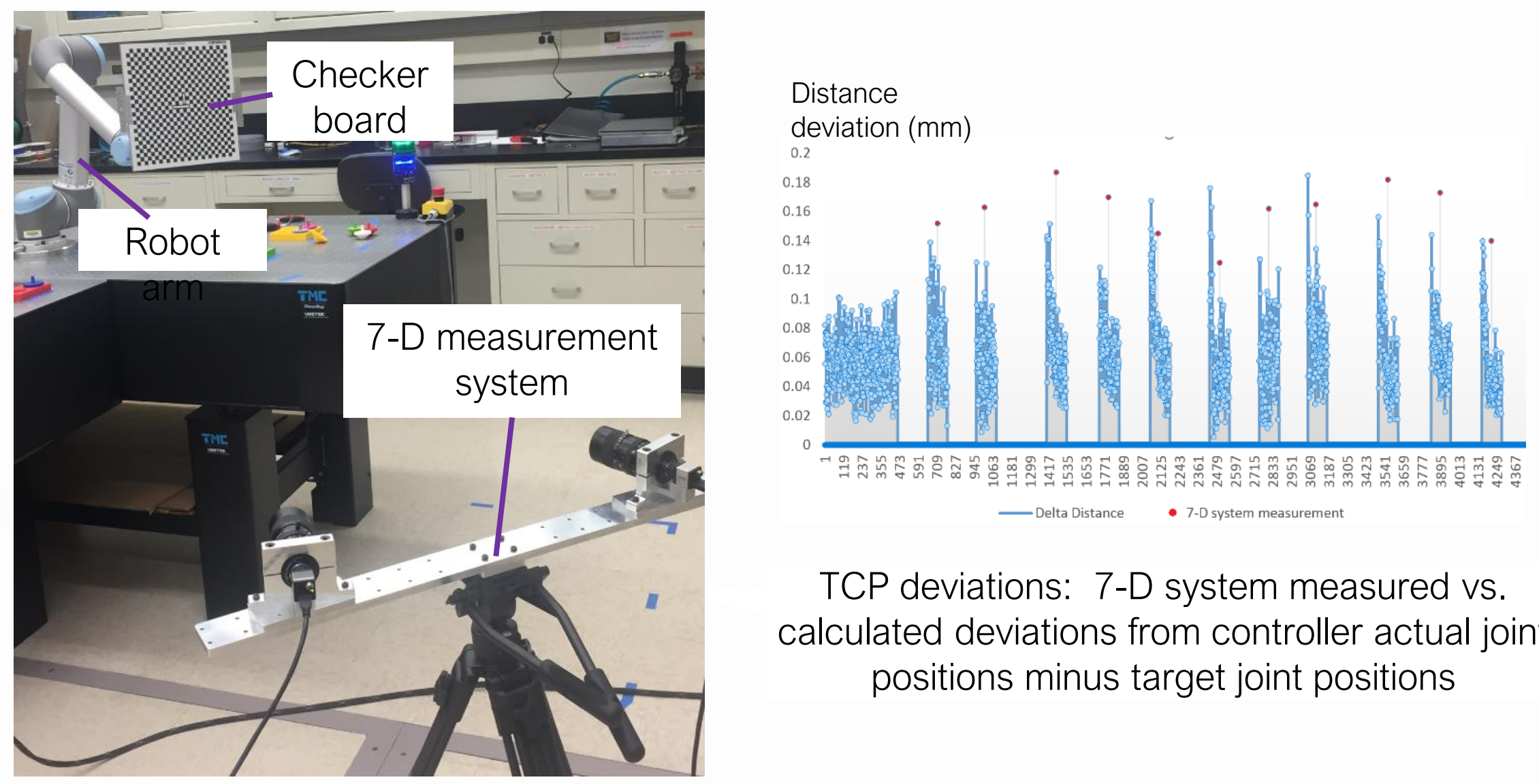

TCP deviations: 7-D system measured vs. calculated deviations from controller actual joint positions minus target joint positions

Reference data set URL:

https://www.nist.gov/el/intelligent-systems-division-73500/cognition-and-collaboration- 
What is the ripple effect in the physical, functional, and informational hierarchies when a process/product degrades?

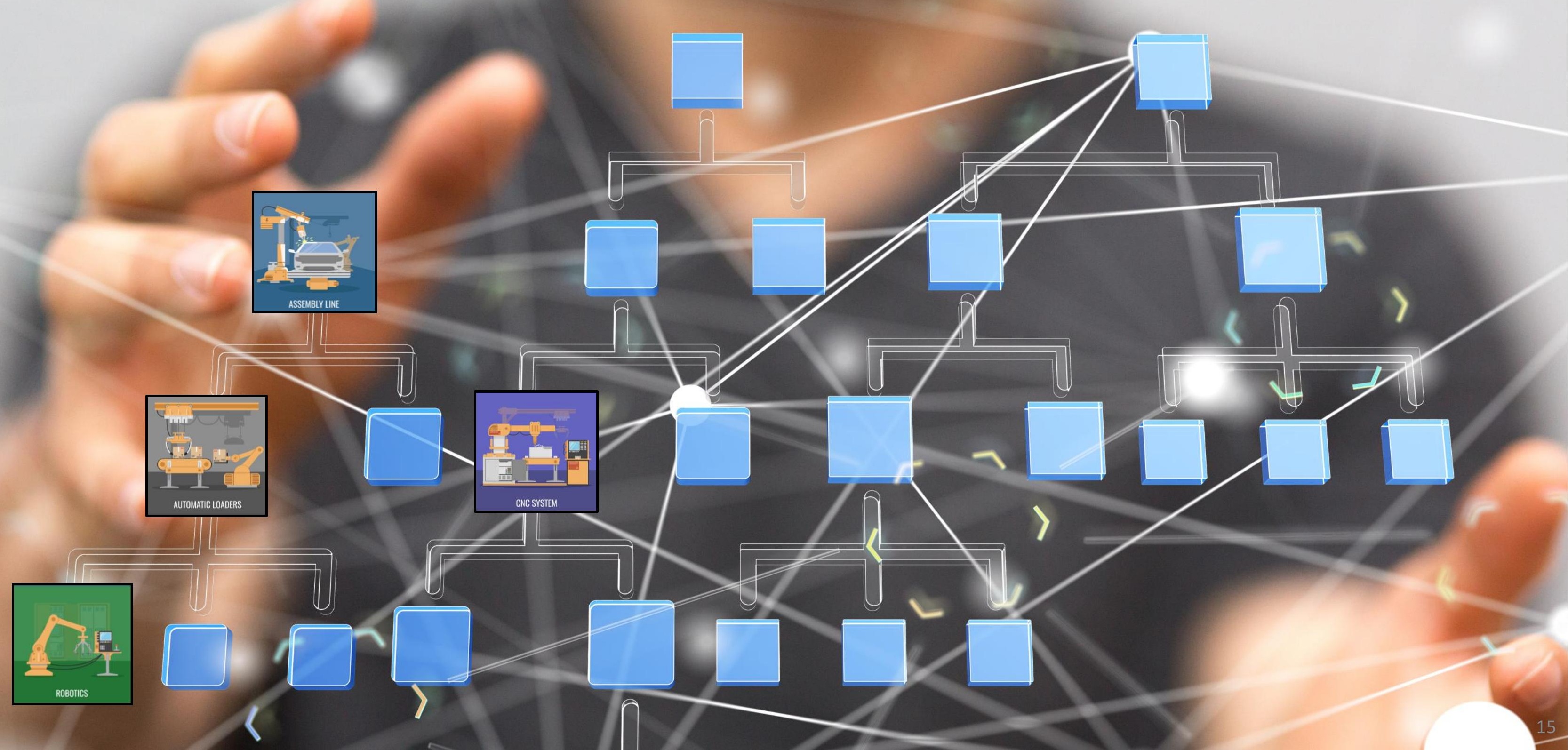


Is there a cost-effective, methodical approach to guide manufacturers through the PHM design and deployment process when you don't know all of the failure modes?

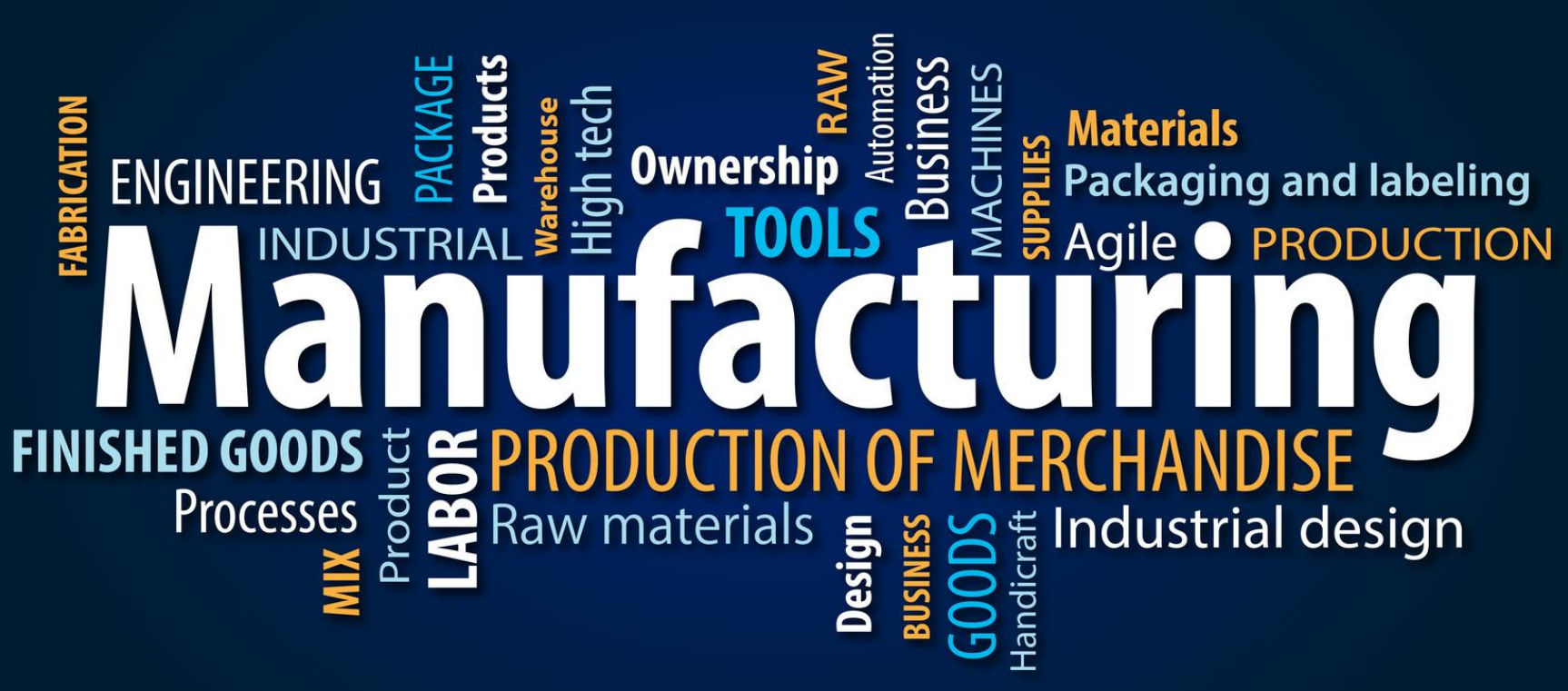

How do you verify and validate such an approach? 


\section{Questions to Answer During PHM Design \& Deployment}

- What physical or task degradation has the potential to impact the metrics I care about most in my process?

What health degradations can impact my quality, productivity, scrap, etc.?

- What data, leading to intelligence, do I need about my process to determine where and when health degradation will occur?

What can be monitored and how?

- How do I prioritize the risk of faults and failures in my system and process? Where should I deploy PHM since I can't put it everywhere?

- How does the health of my physical system, and its constituent elements, influence the health of my process?

How can I map the relationships between the physical and functional to better understand my process? 
Steps 1, 2, 3

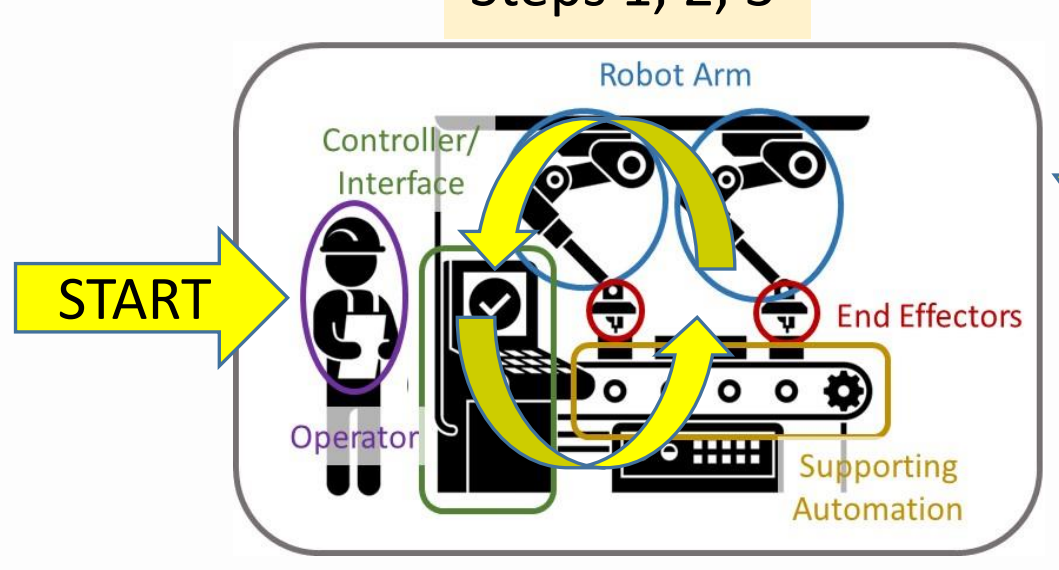

Physical and Task Decomposition,
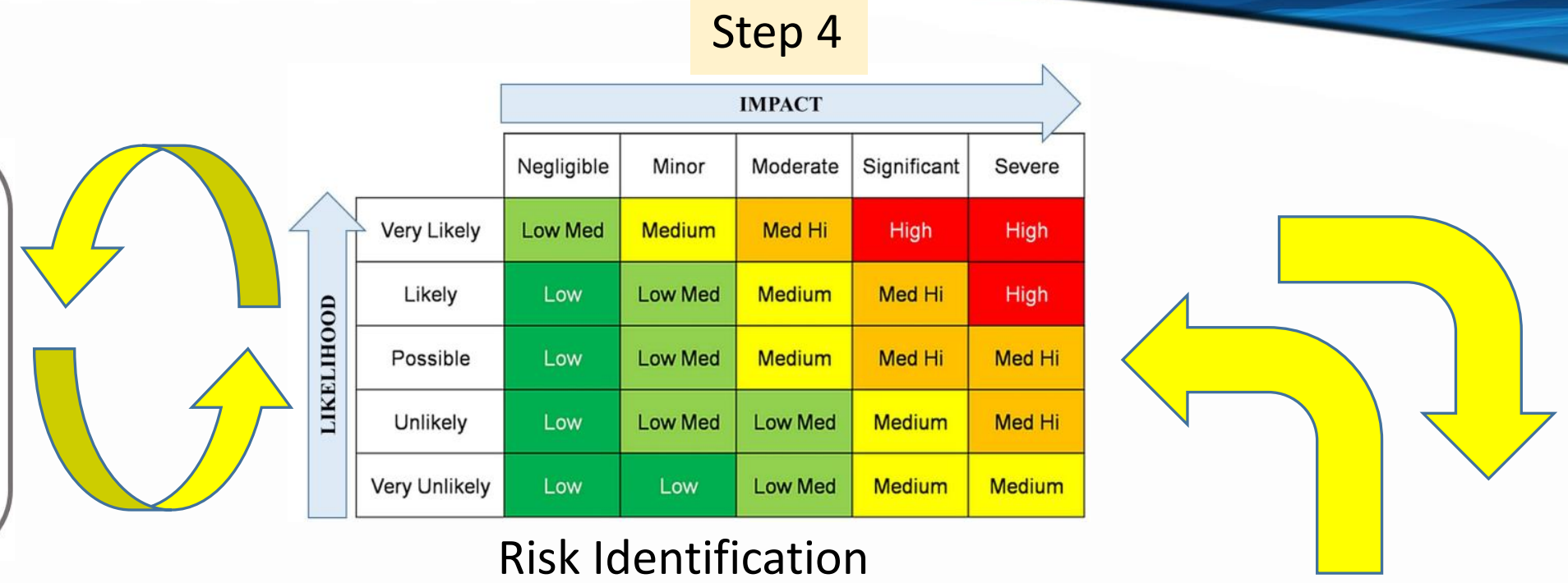

Process and Task Metric ID with Relationships

Risk Identification

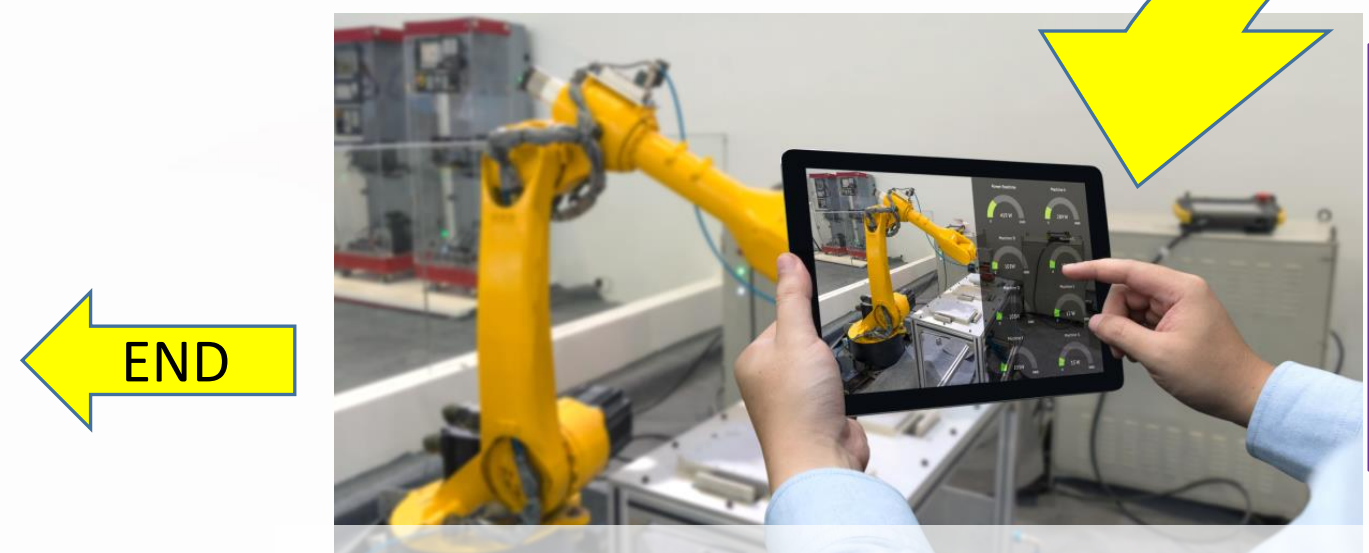

Physical Element Metric ID, Relationship Mapping and Quantification

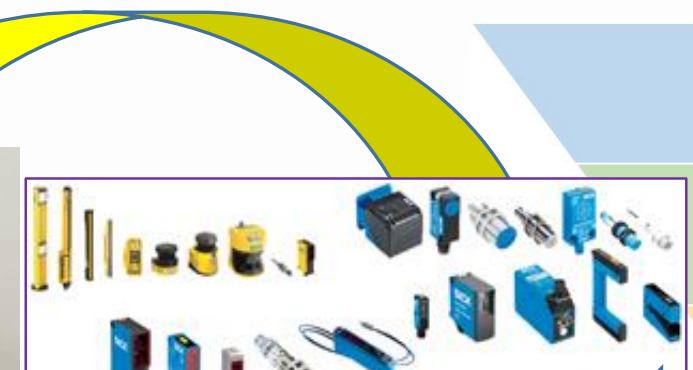

2 1 18 and is $E$ Data to Collect and Collection Method Step 6

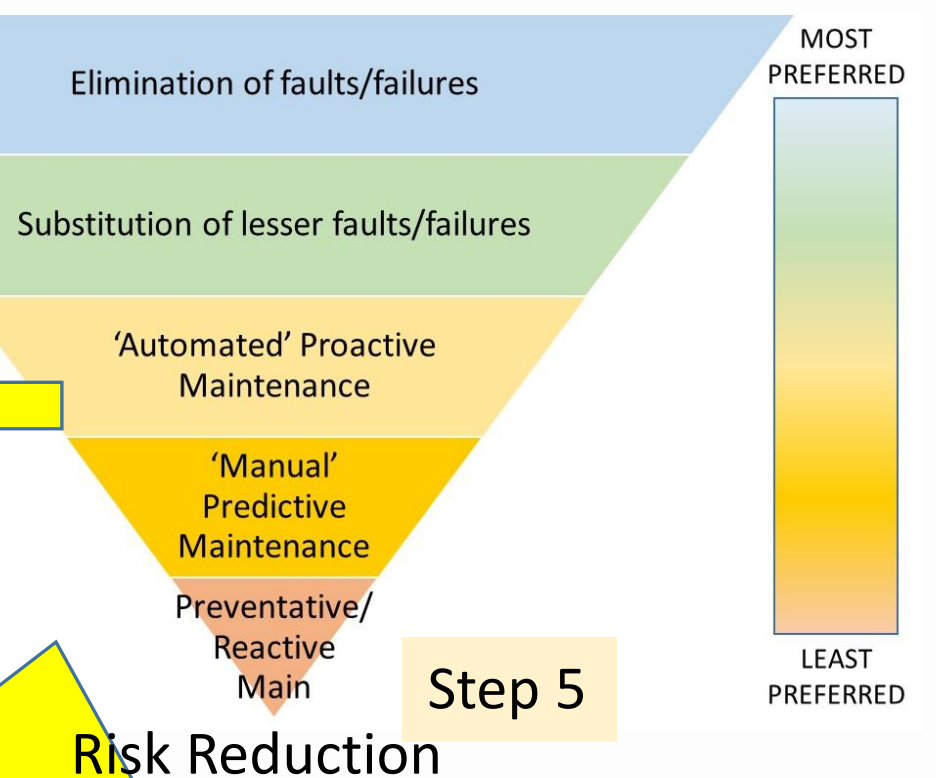

Steps 7, 8

\section{Risk Reduction}




\section{Next Steps...}

- Updating our research efforts to better reflect changing needs of industry

- Further development of test methods and performance metrics

- Strategic collaborations with industry to pilot test methods to get and give feedback

- Greater understanding of common configurations

- ATTEND Friday's ASME's Standards Meeting

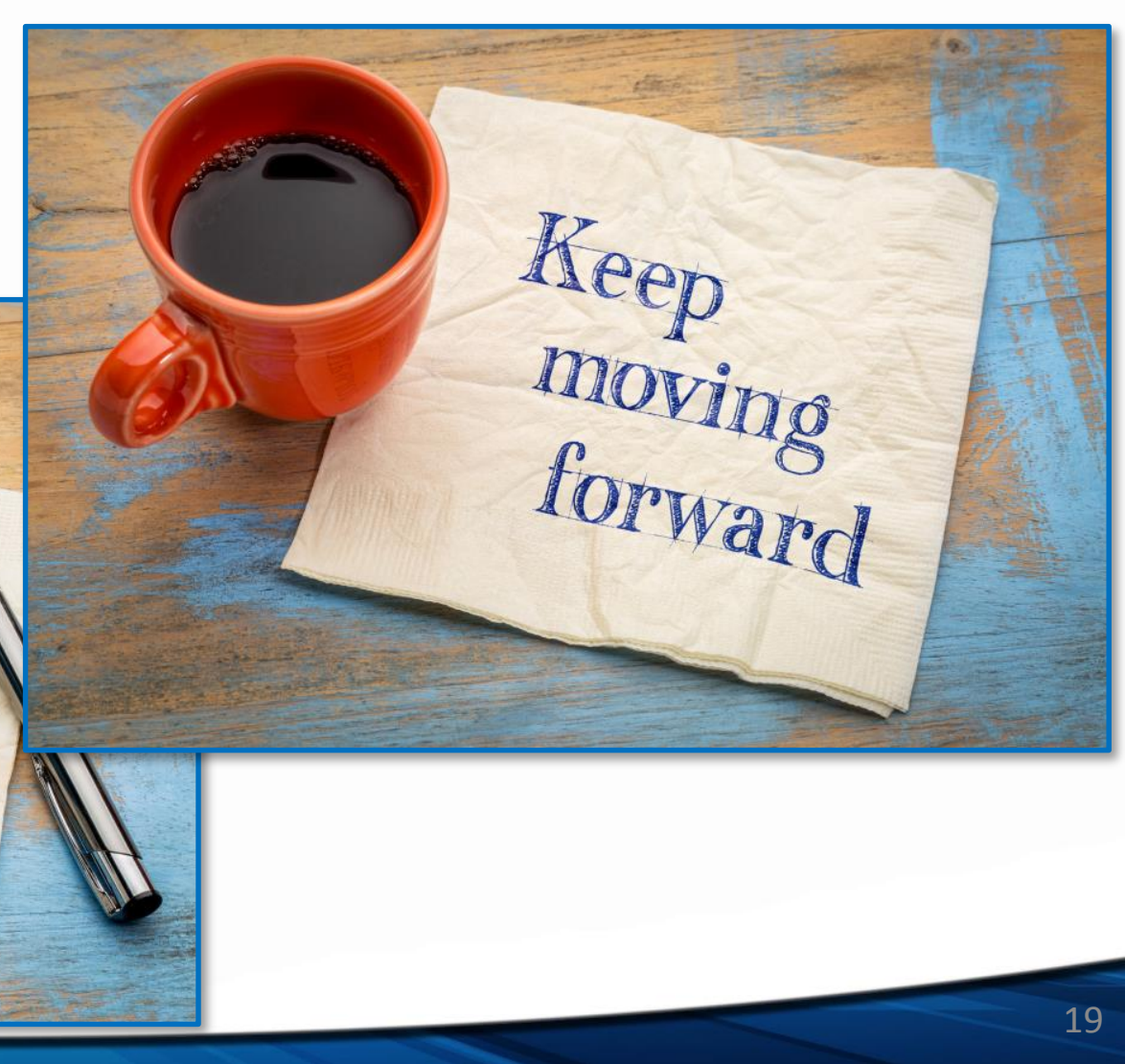

Surround
Vourself with the right people
Keep

moving

forward 
Brian A. Weiss Intelligent Systems Division brian.weiss@nist.gov (301) 975-4373

\section{NIST}

100 Bureau Drive, MS 8230 Gaithersburg, MD 20899

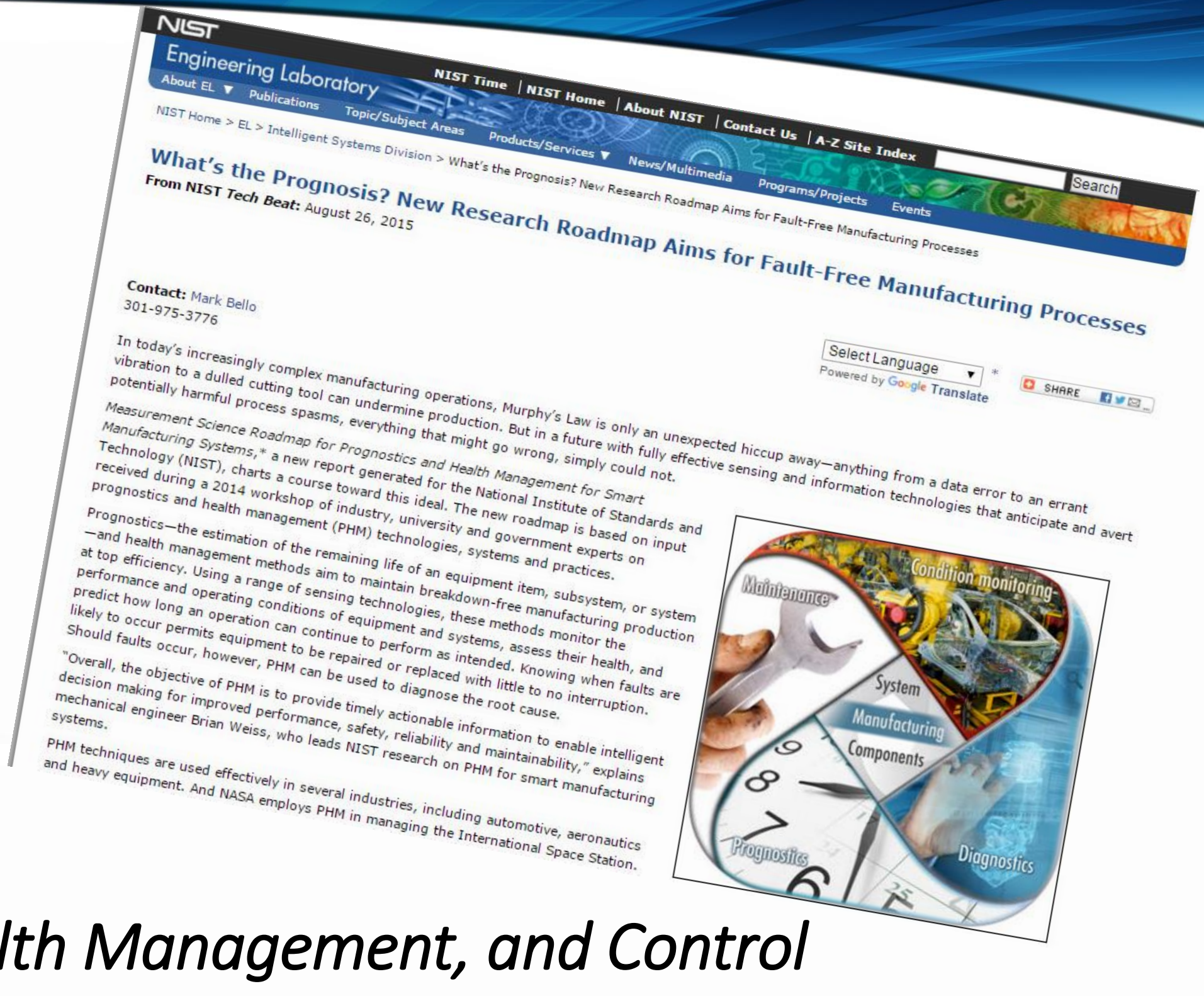

www.nist.gov/el/isd/ks/phmc.cfm 


\title{
Challenges in Standards for a Model-Based Enterprise
}

\author{
Thomas Hedberg, Jr., P.E. \\ Systems Integration Division, Engineering Laboratory \\ National Institute of Standards and Technology
}

Industry Forum: Monitoring, Diagnostics, and Prognostics for Manufacturing Operations 10 May 2018 
B.L.U.F.

Industry needs open architectures to support the "brownfield" realities of manufacturing and allow interconnectivity across decentralized systems. Model-Based Enterprise would address this industrial need by coupling existing technologies with trusted systems, Internet of Things, Big Data, and Artificial Intelligence to enable advanced variant configuration - allowing Industry to be agile and flexible enough to manufacture closer to the end user, in varying lot sizes, for the first time. 


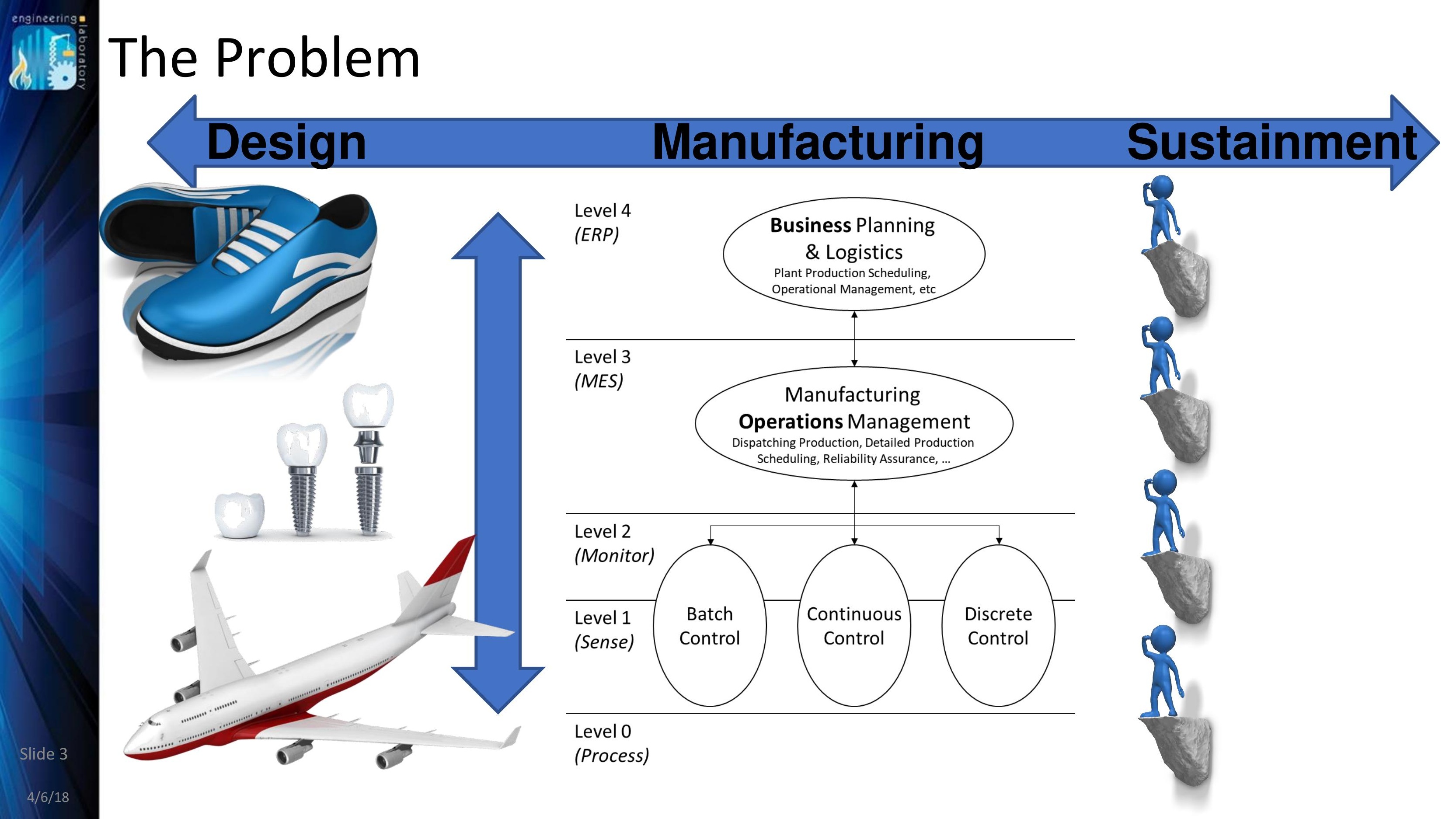


"There are problems that manufacturing companies have been trying to solve for decades. How do I make my inventory management more efficient? How do I match the supply of product that I'm making with the actual demand?"

Rick Smith, co-founder of Fast Radius On the need for a Digital Supply Chain 
"It's really an ecosystem of technologies that work together. It's not just one thing, it's many things that are working together."

Alan Amling, VP of strategy at UPS On IloT in Logistics 
"What's changing now is the speed required to deliver a lot size of one. Consumers are no longer willing to wait 15, six, or even three months. Innovation is moving from mechanical features to embedded software, driving a need to condense design cycles - and a convergence of silos. Non-integrated, non-real-time solutions won't support the next wave."

John McNiff, VP of Solution Management at SAP On Configurable, Personalized Products 
How do I match product needs to process capabilities?

In the context of PHM, How do I know when to sacrifice system capacity today to ensure capacity and availability of my systems tomorrow? 
- Standards for Product Definitions

- What is the product?

- What are the feature requirements?

- Standards for Process Definitions

- What resources exist?

- What capabilities do the resources have?

- Methods for matching and/or aligning the definitions

- How do the available capabilities satisfy the feature requirements? 
Three Classes of Standards

- Practice and Specification

- ASME Y14 \& ASME MBE

- ISO TC 10 / TC 213

- ISA-95

- Data and Information

- ISO 10303 (STEP)

- ISO 32000 (PDF) / ISO 14739 (PRC)

- ISO 14306 (JT)

- MTConnect

- ANSI/DMSC QIF

- OAGIS

- opcua
- Modelling

- PMML

- MIMOSA

- AutomationML

- ISO/ AWI 23247

- $\quad$ ASME V\&V 50

- OMG SysML

- OMG UML 
The Problem: Redux

\section{Design}

Native CAD

STEP

PDF / PRC

ASME Y 14

QIF $_{\text {MBD }}$

\section{Manufacturing}

Sustainment

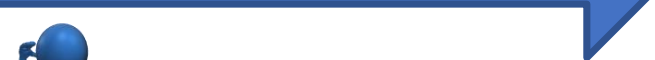

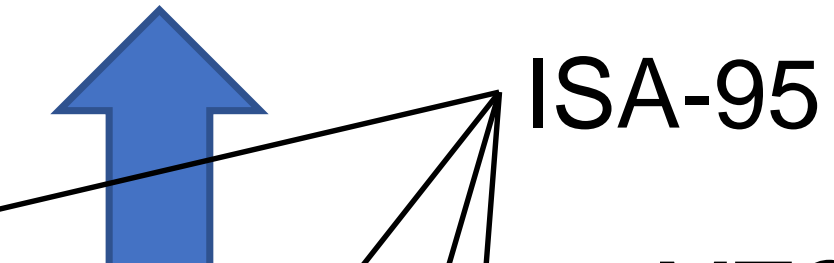

MTConnect

PMML

ISO/ AWI 23247

QIF

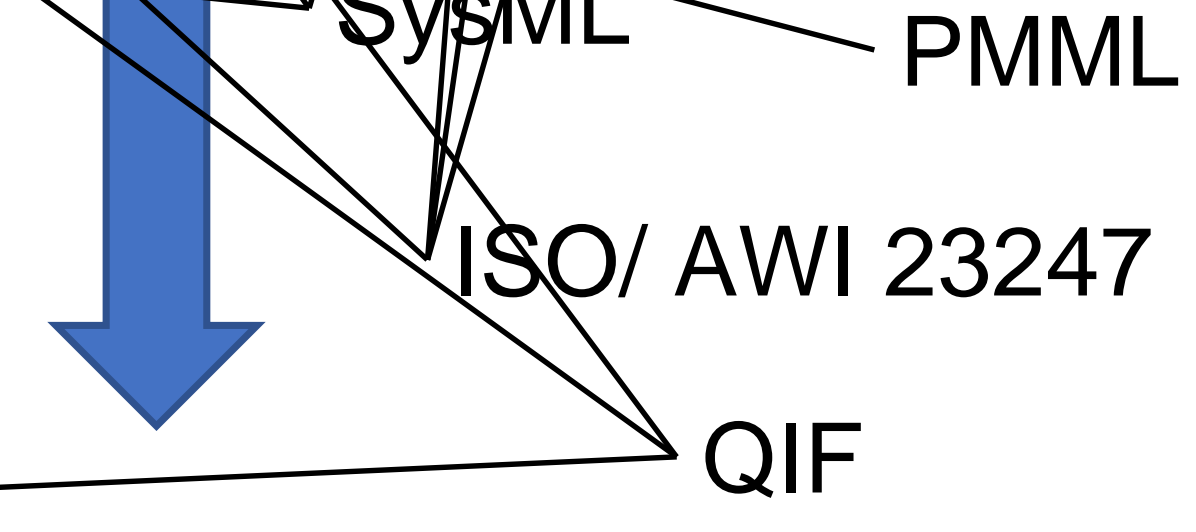



going to do?

\section{ENTERPRISE}




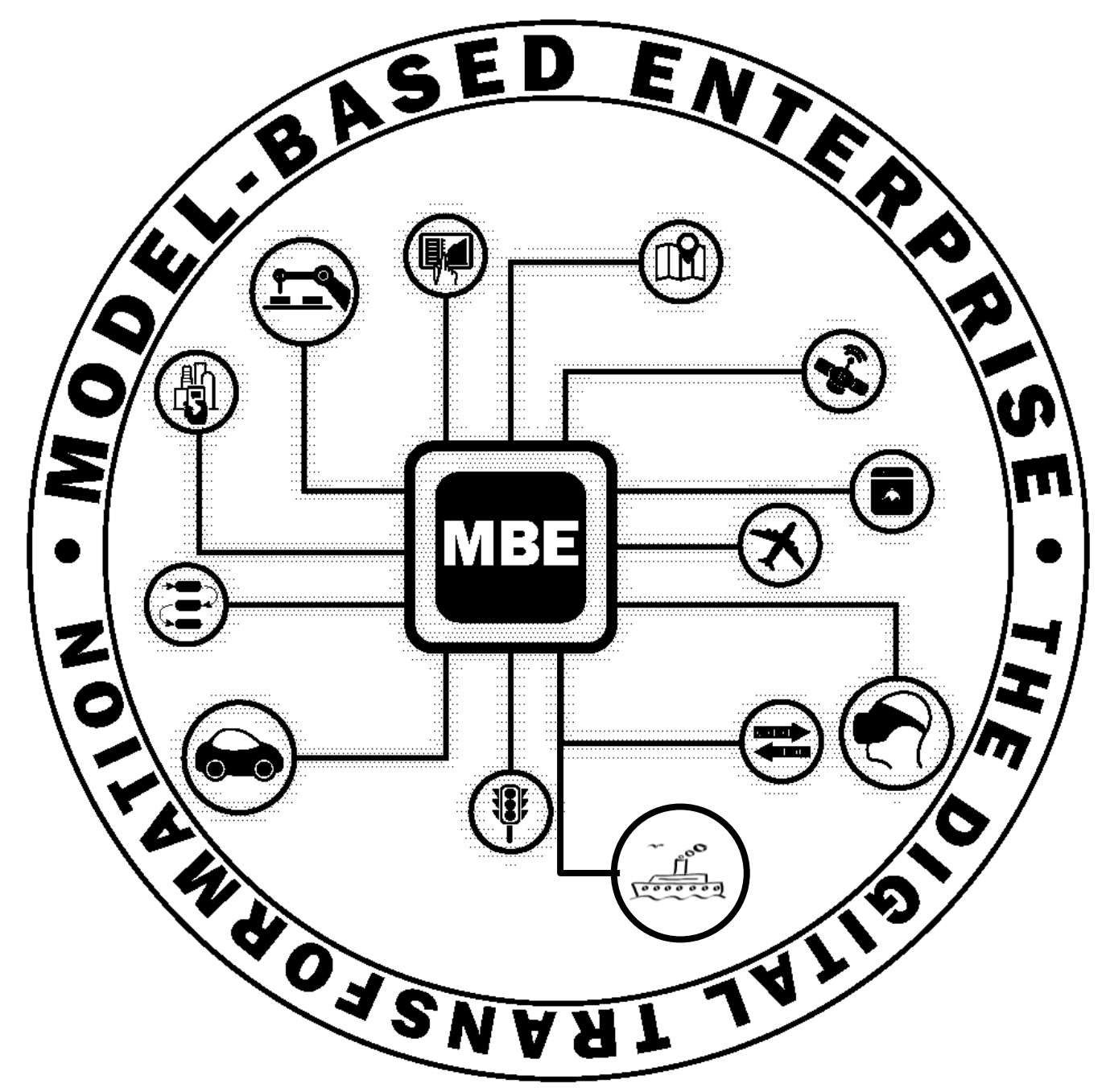

Enabling đel control acrossutpreupsoding lofpeyalien ris basedron outcomes 


\section{Questions? Save 'em for the panel! ;-)}

Thank you for your kind attention!

Thomas Hedberg

thomas.hedberg@nist.gov

Digital Thread: https://go.usa.gov/xNP8x

SMS Test Bed: https://smstestbed.nist.gov

My Publications: https://go.usa.gov/xNP8R 


\title{
Visualization Tools for PHM: Metrics of Effective HMI
}

\author{
Jeremy A. Marvel, Ph.D. \\ Project Leader, Performance of Collaborative Robot Systems \\ Co-PI, Tools for Collaborative Robots in SME Workcells \\ U.S. Department of Commerce \\ National Institute of Standards and Technology \\ Engineering Laboratory, Intelligent Systems Division
}




\section{Requisite Disclaimer}

Commercial equipment and materials are identified in order to adequately specify certain procedures. In no case does such identification imply recommendation or endorsement by the National Institute of Standards and Technology, nor does it imply that the materials or equipment identified are necessarily the best available for the purpose. 


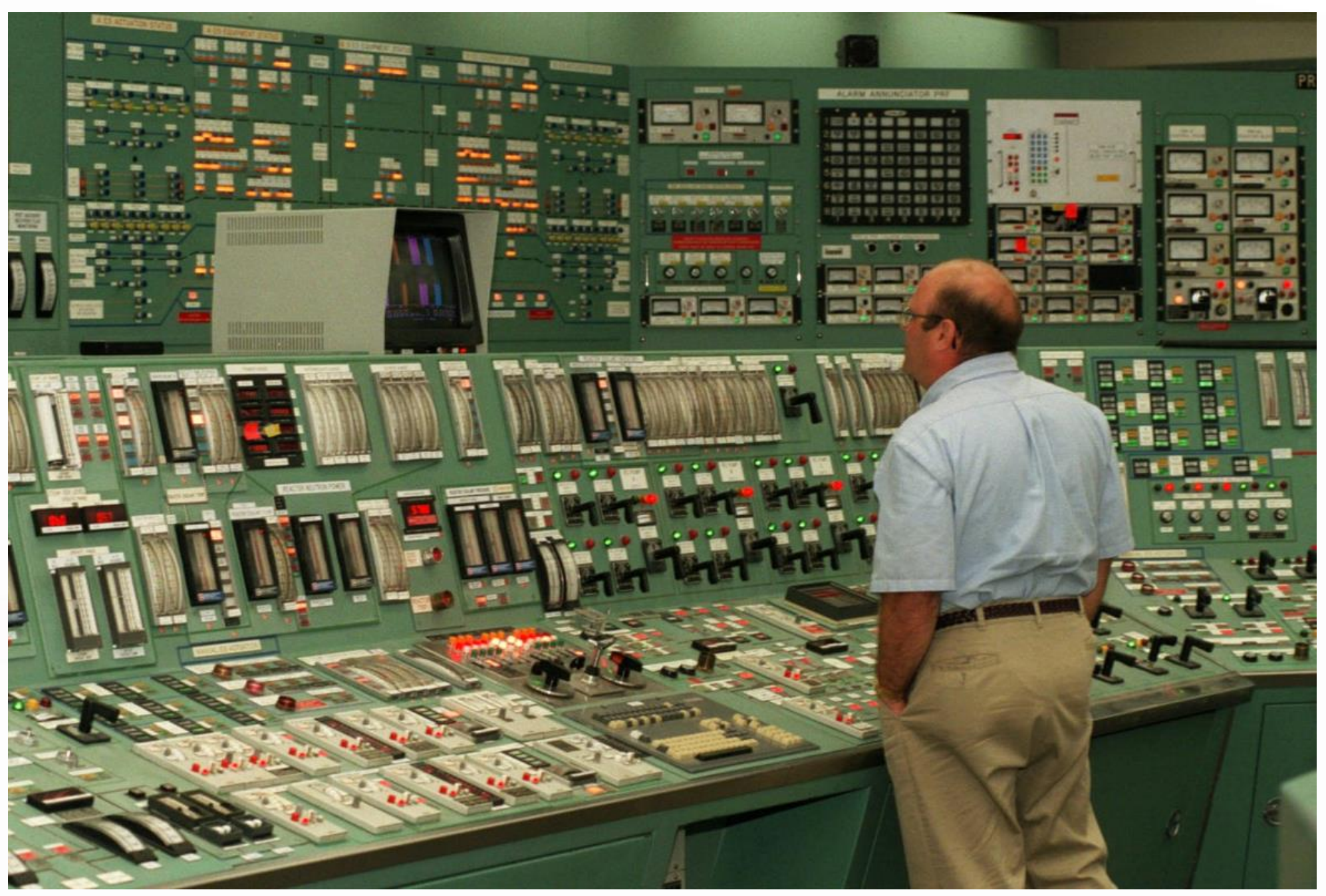

Image source: Baltimore Sun d. 


\section{State-of-the-Art in HMI: Robotics}

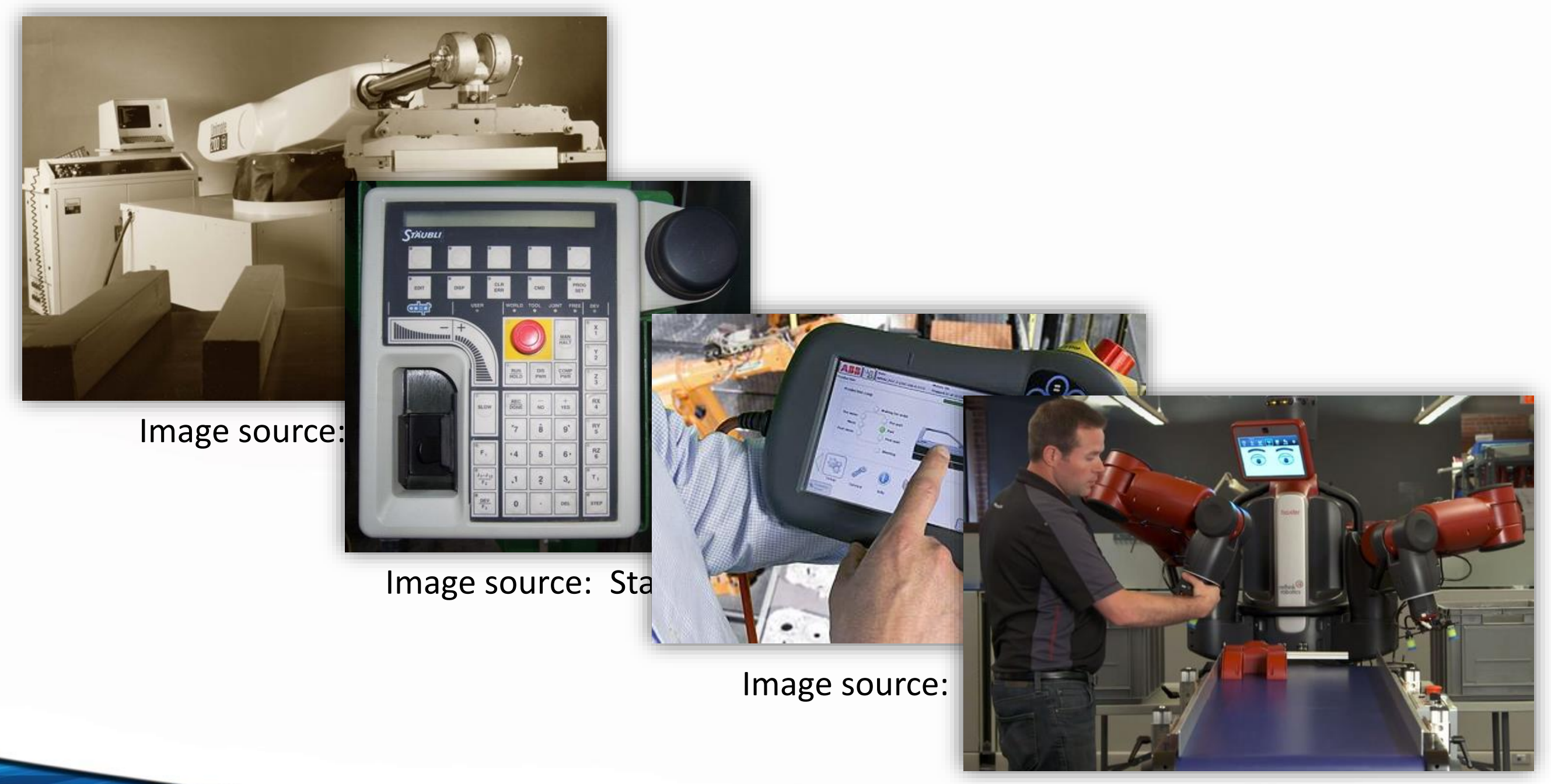




\section{Degrees of Interface Separation}

- Process interface: Easy-access buttons and lights

- Start, stop, pause functions

- Move up/down, left/right

- Light towers for quick status updates

- Auxiliary interface: PLC

- Limited robot status via DIO (safety system status, enable state, program state, etc.)

- Principal interface: Teach pendant

- Robot position (joint \& Cartesian), forces and torques, etc.

- Program position

- Error codes

- Maintenance cycles (backup battery, mastery, etc.)

- Workcell level: PLC

- Coordinate multiple PLCs/controllers

- Provision of workcell safety

- Factory floor level: Fleet management software

- Coordinate flow of parts/materials to different workcells

- Monitor line status and dispatch maintenance

- Monitor and manage resources

Image source: Babylonbee.com

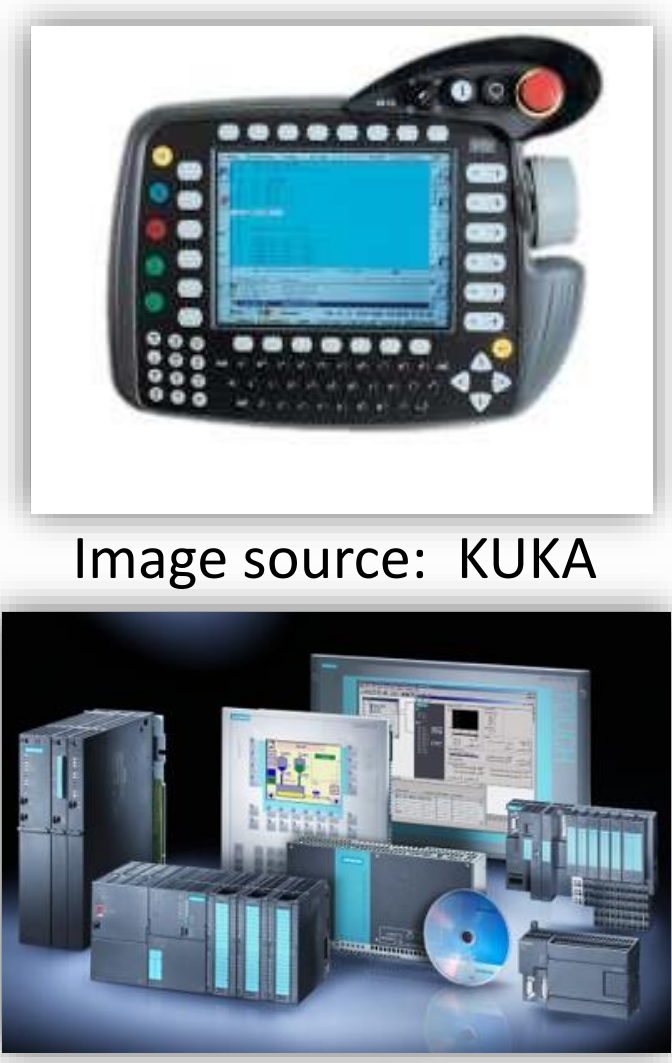

Image source: Siemens 


\section{Interfaces Dictate Interaction}

- Purpose of interface:

- Information presentation

- System/process control

- Consumer goods are human-centric

- Ease of use and intuition

- Clear and contextual feedback in real-time

- Reduction of user strain

- Cost reduction, not operator's needs, drive industry HMI designs*

- Generational differences result

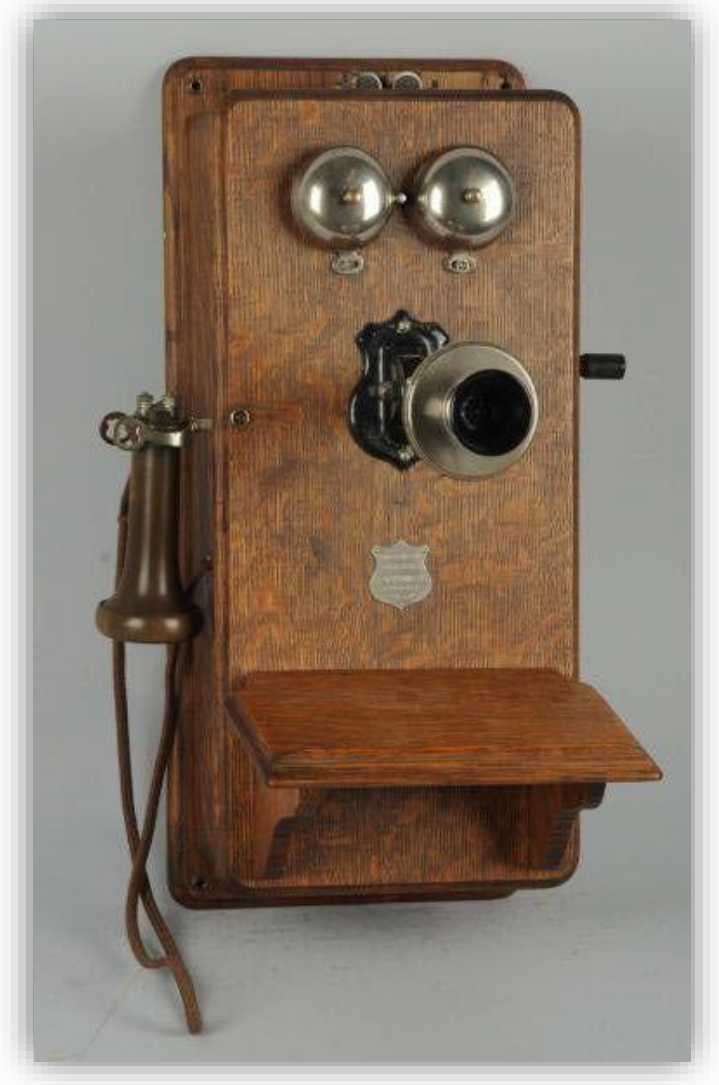

- Younger generations put off by antiquated interfaces 


\section{Physiological Bases}

- Fitts' Law

- Time required to move one's hand to a target location

- Goal-oriented thought cycle:

- Form a goal

- Choose and execute actions to make progress

- Assess impacts of selected actions

- Repeat previous two steps until goal achieved

- Change blindness

- Inattentional blindness

- Gestalt theory of perception

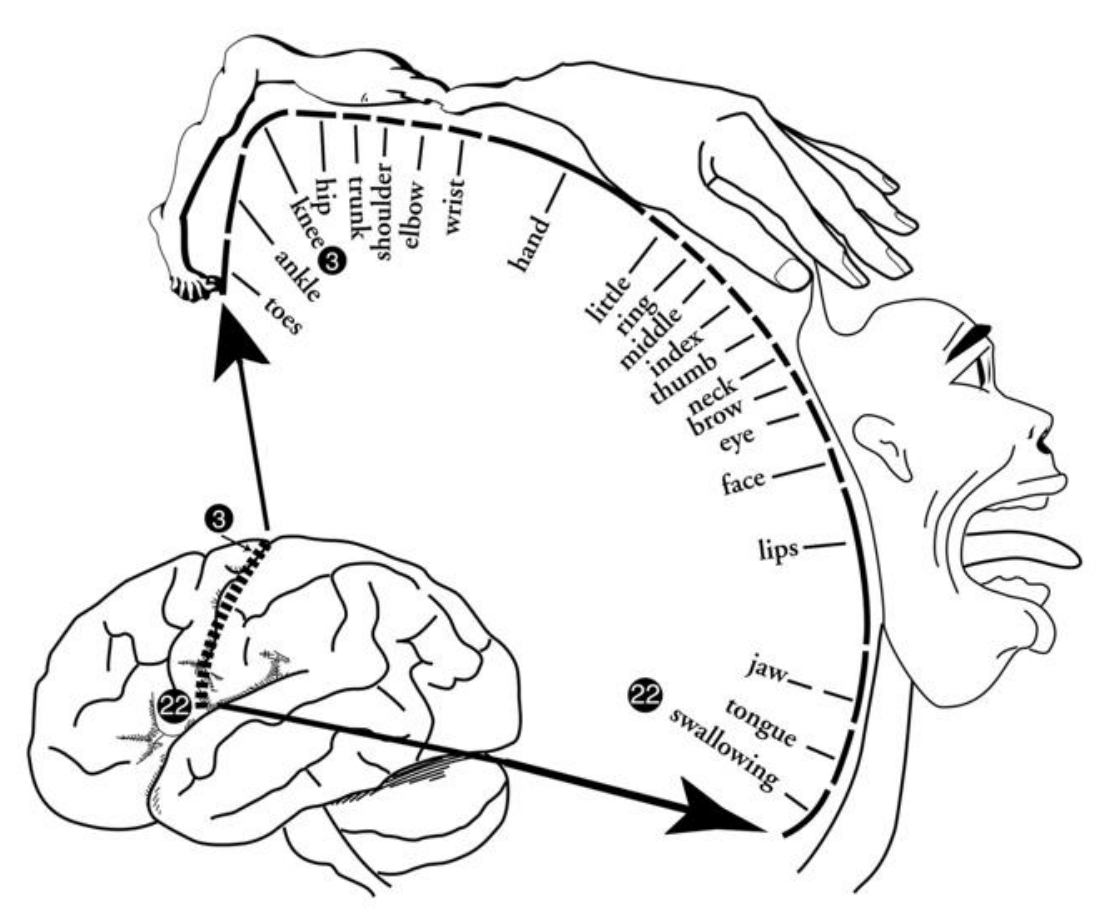

- Association of objects clustered/moving together

- People draw conclusions in absence of structure and fill in gaps

- Poor management of information leads to information disassociation

- Uncertainty principal

- Decision time increases as a function of the uncertainty of decision, or the number of alternative answers 


\section{Metrics}

- Software quality

- ISO/IEC 25010:2011

- Quality in use - evaluate impacts and outcomes resulting from the use of the system

- Product quality - characterize system by its properties

- Interface

- ISO 9241-210:2010 (Human-centered design for interactive systems)

- User-based testing

- Inspection-based evaluation

- Impacts of use:

- Learning time, expert use time, error cost, functionality

- Information quality

- Response to presented information correct?

- Time lapsed between presentation and response

- Power Law of Practice

- Time to perform tasks decreases as a function of repeated use

- Clarity and conciseness of message

- User response

- Mental effort (impact of time, stress, team diversity) 


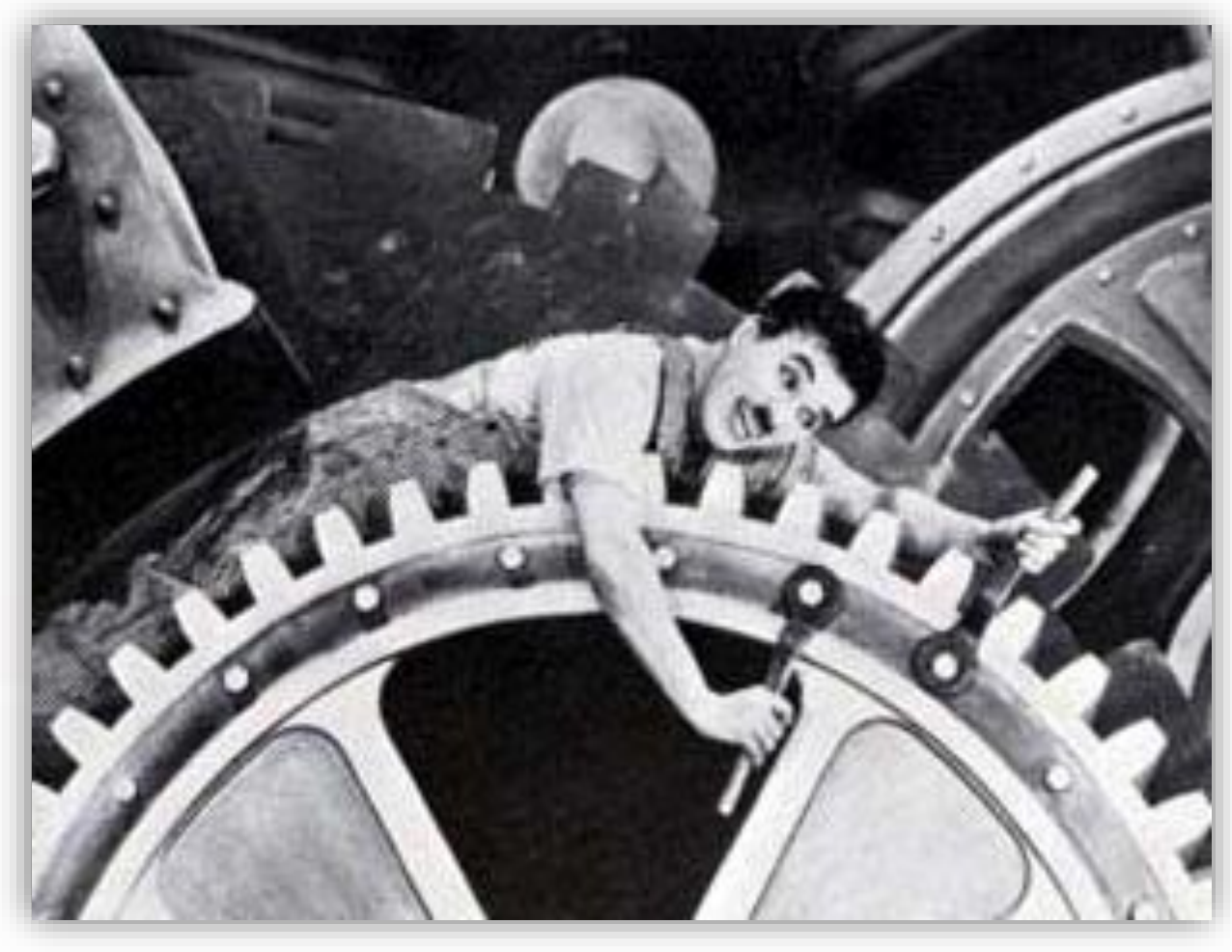

Image source: United Artists (C) 


\section{NLT}

National Institute of

Standards and Technology

U.S. Department of Commerce

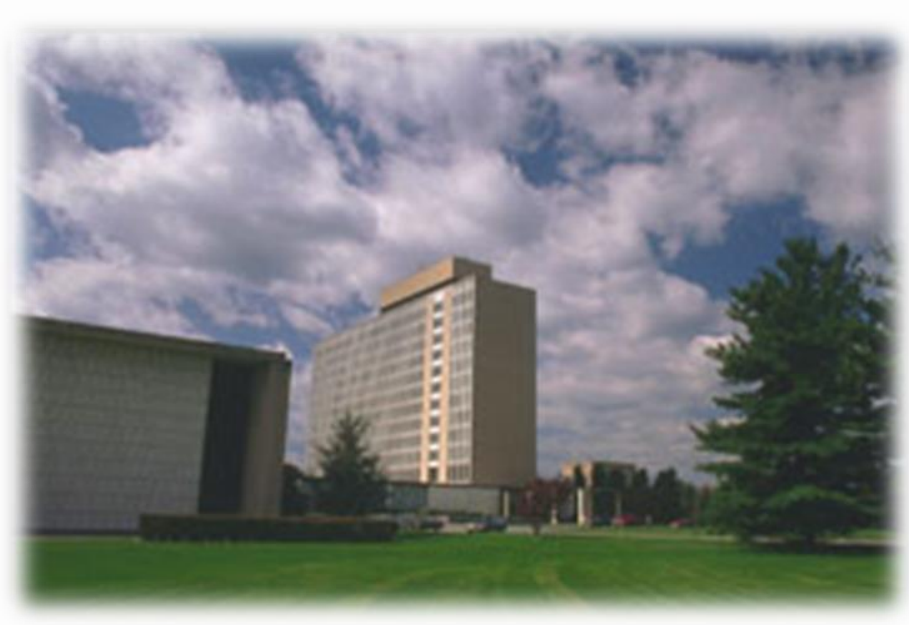

J eremy A. Marvel, Ph.D.

\section{Computer Scientist}

PI, Performance of Collaborative R obot Systems

Co-PI, Tools for Collaborative Robots within SME Workcells

\section{U.S. National Institute of Standards and Technology} 100 Bureau Dr., Stop 8230

Gaithersburg, Maryland, 20899, USA

+1 3019754592

jeremy.marvel@ nist.gov 


\section{Demystifying Today’s Artificial Intelligence}

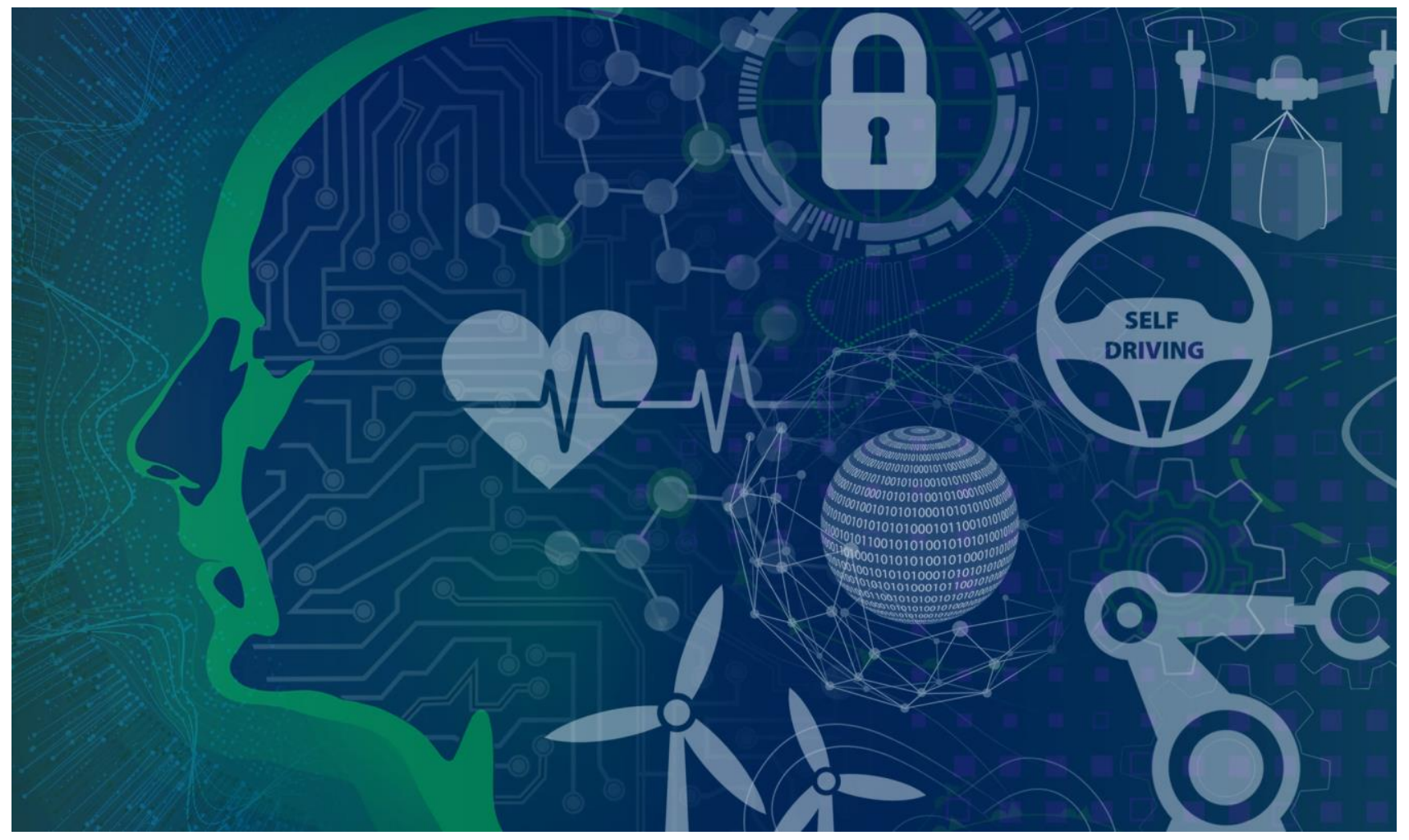

\section{Michael Garris}

Senior Scientist / National Institute of Standards and Technology

Co-Chair / National Science and Technology Council,

Subcommittee on Machine Learning and Artificial Intelligence 


\section{What's all the fuss?}

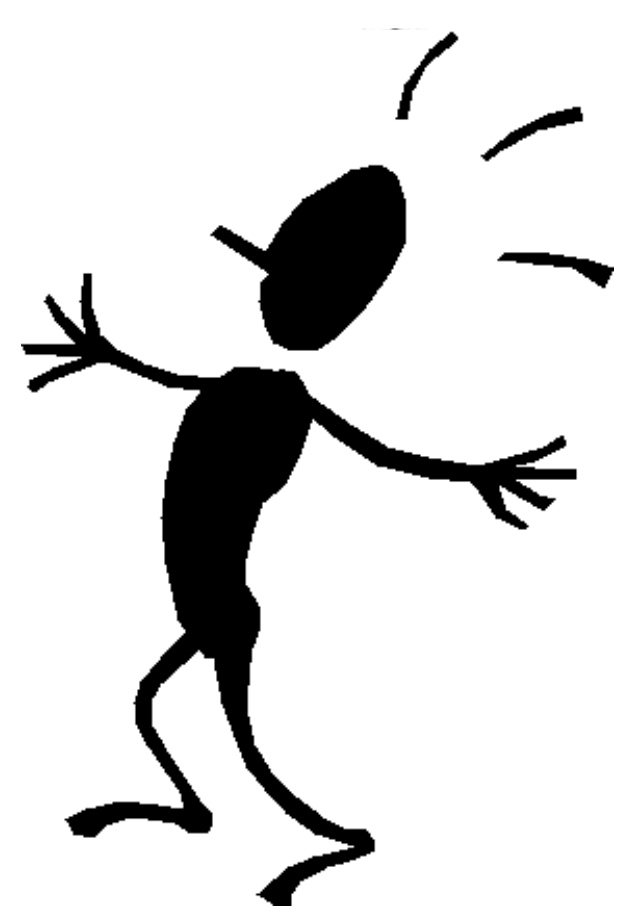




\section{Emerging Technology}

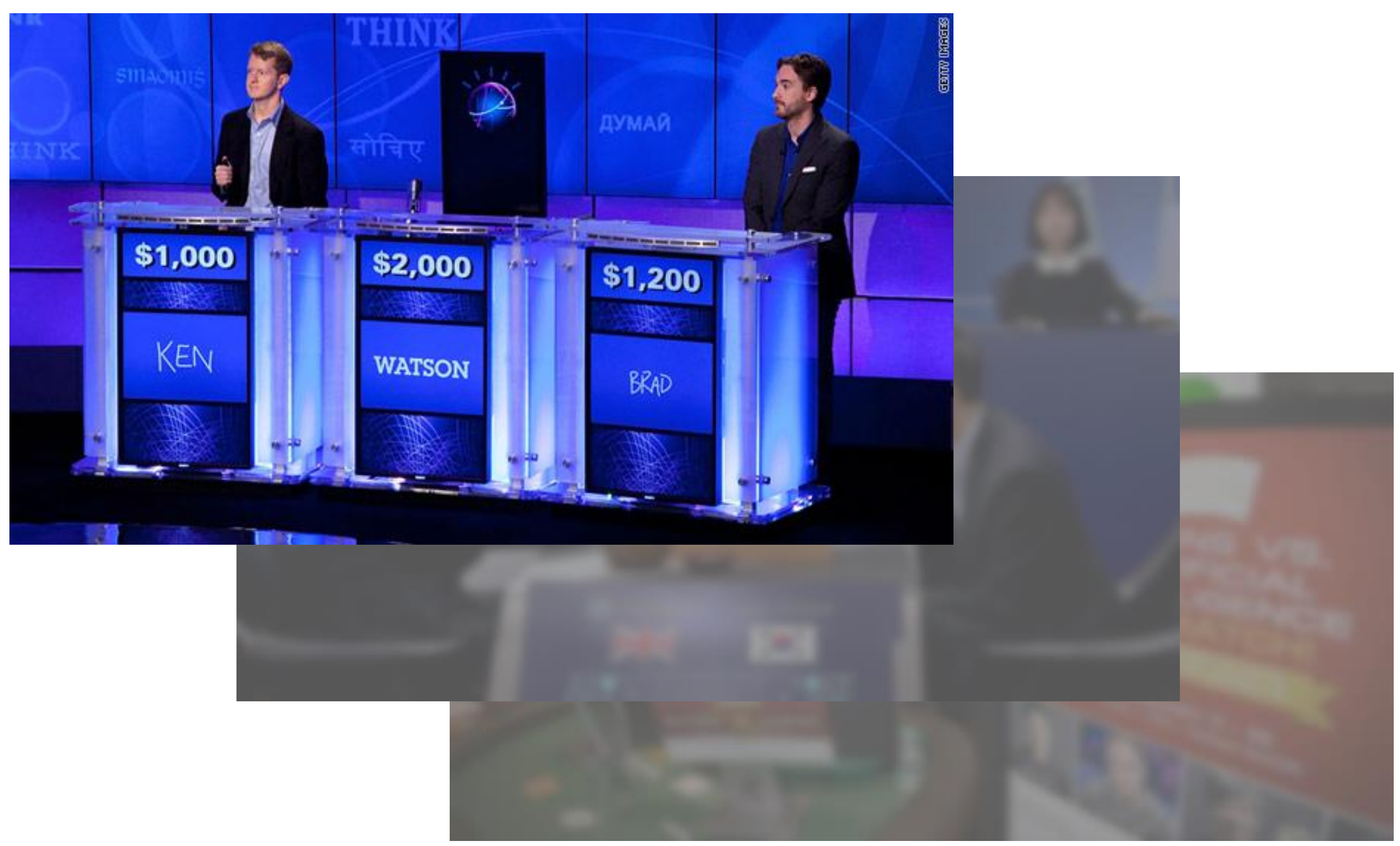




\section{Emerging Technology}

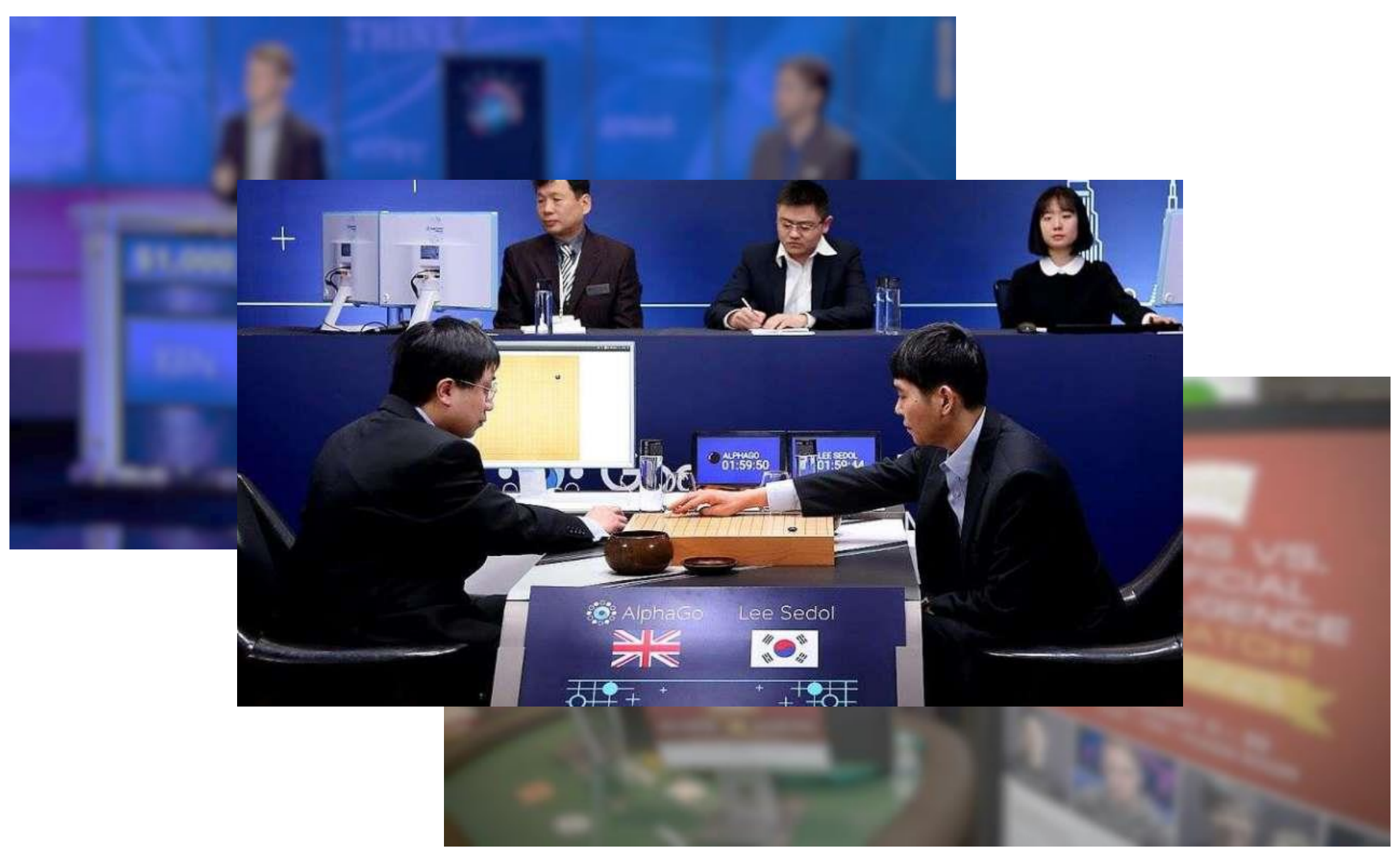




\section{Emerging Technology}

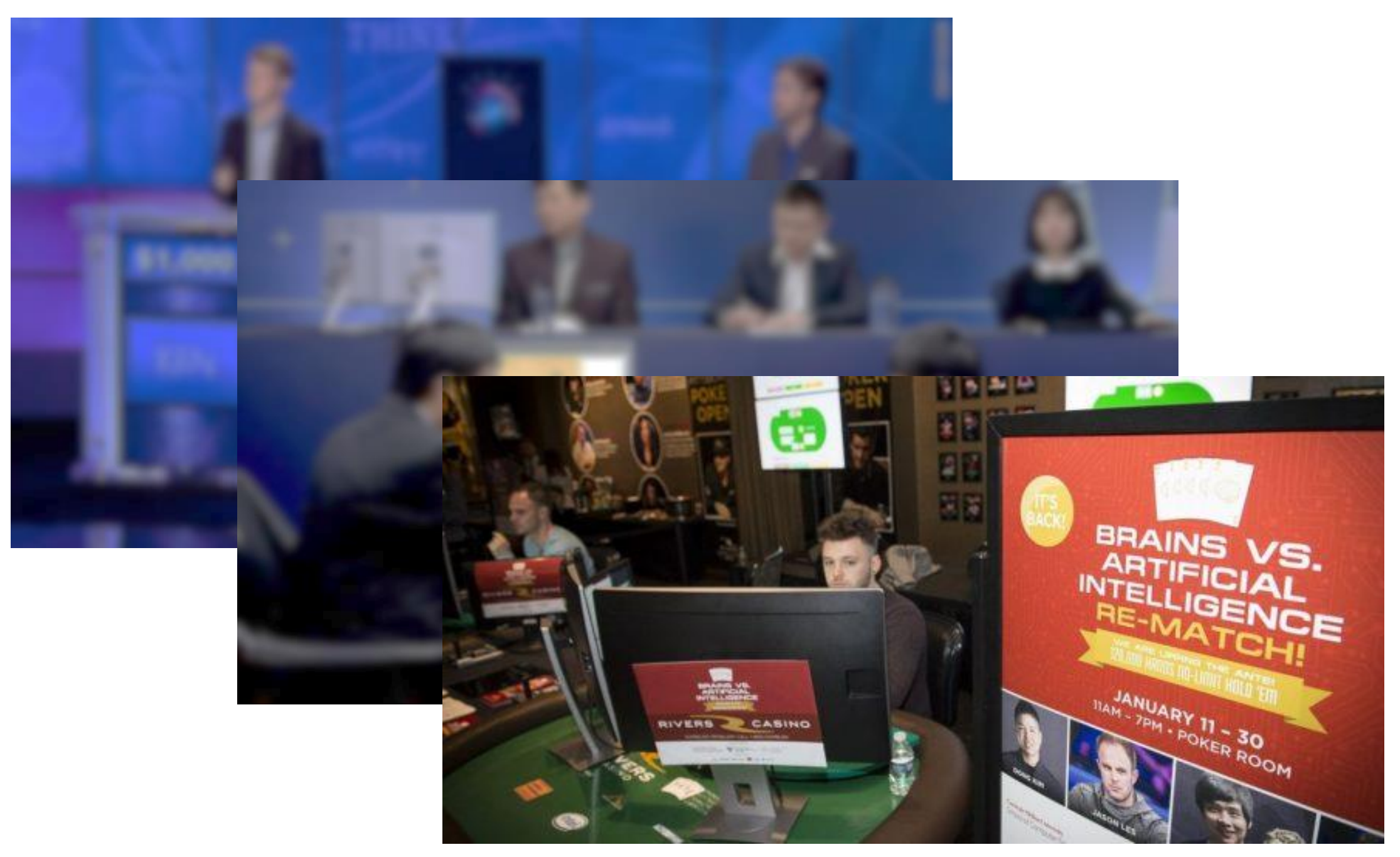




\section{Emerging Technology}

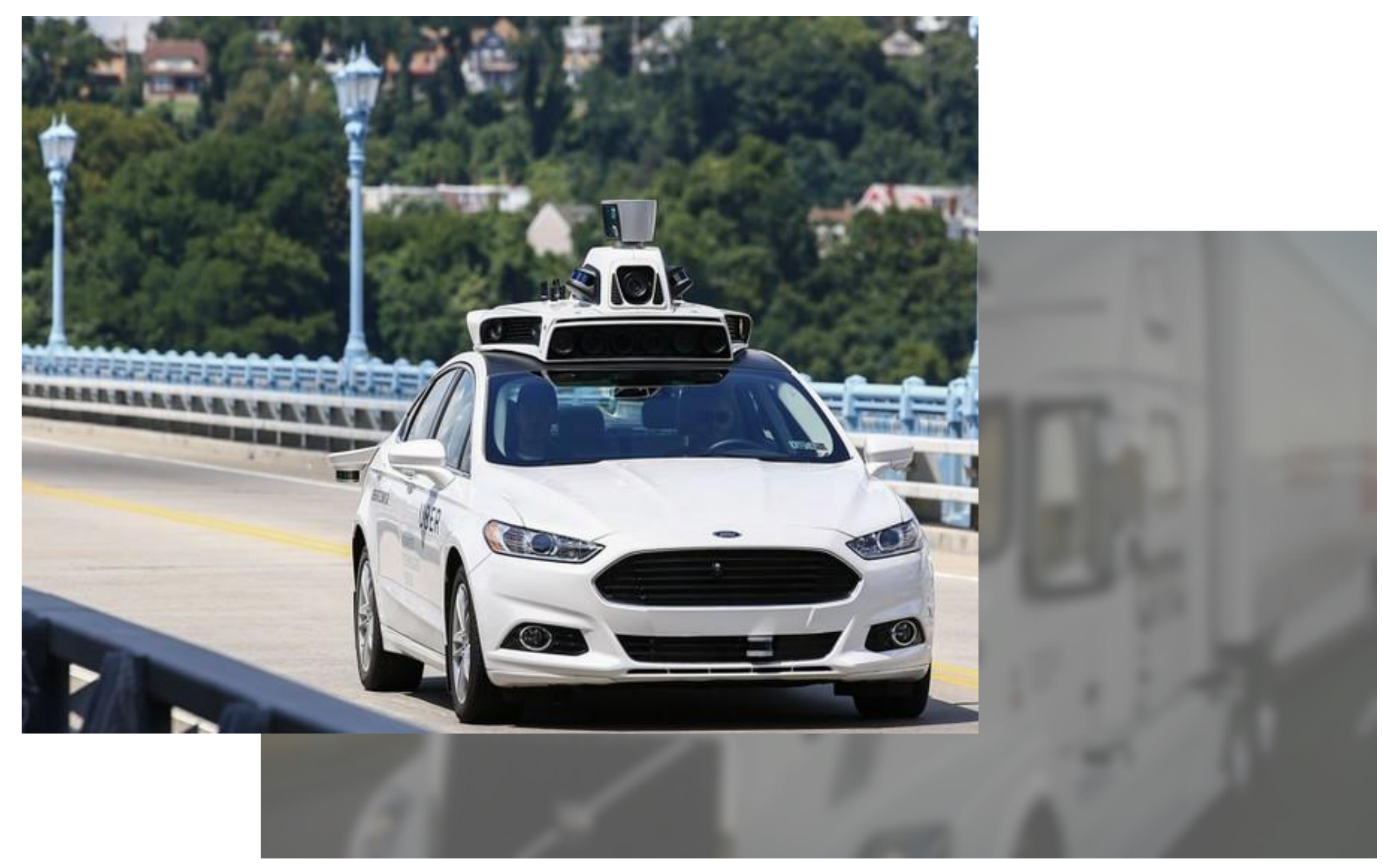




\section{Emerging Technology}

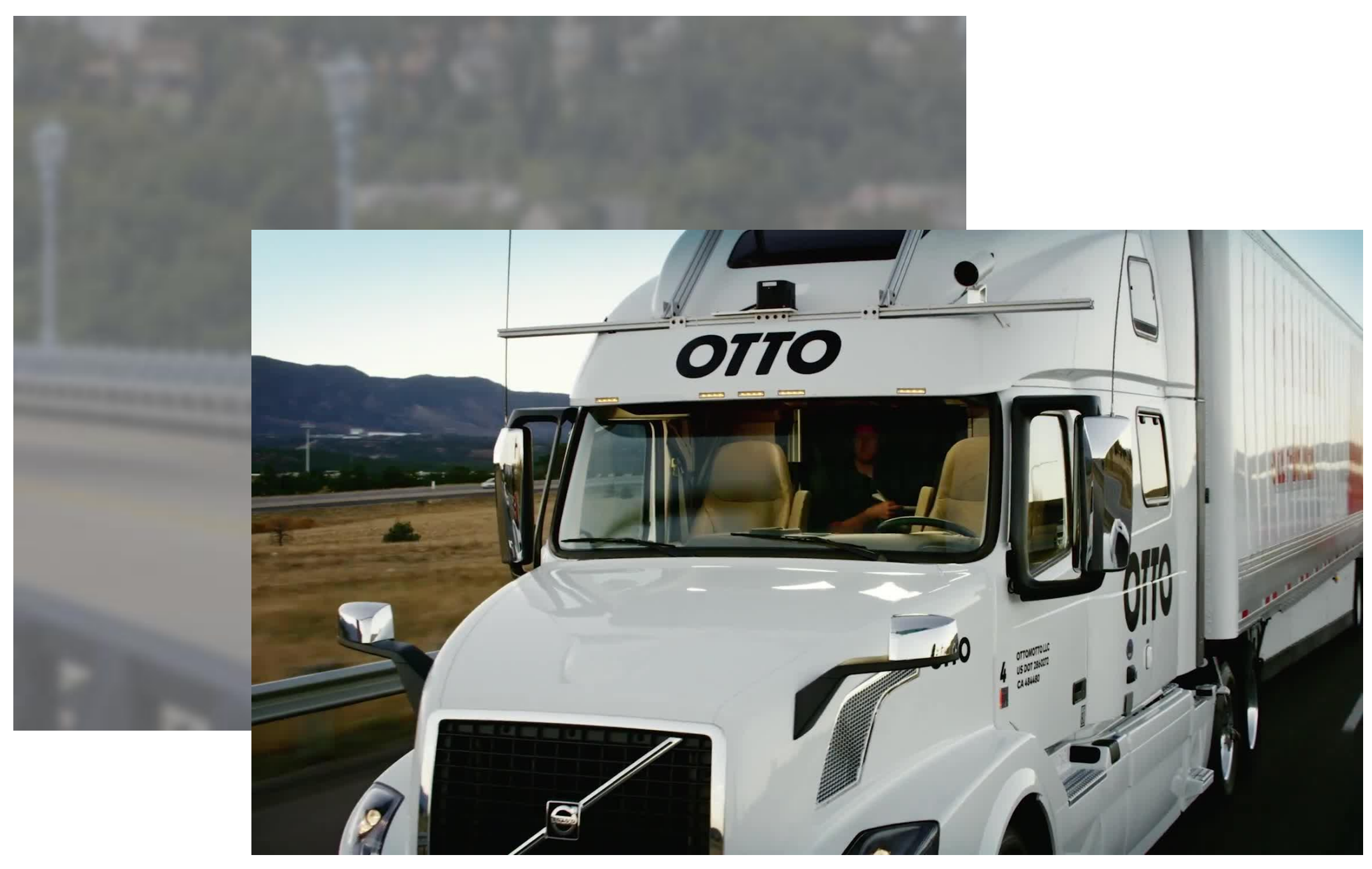




\section{Emerging Technology}

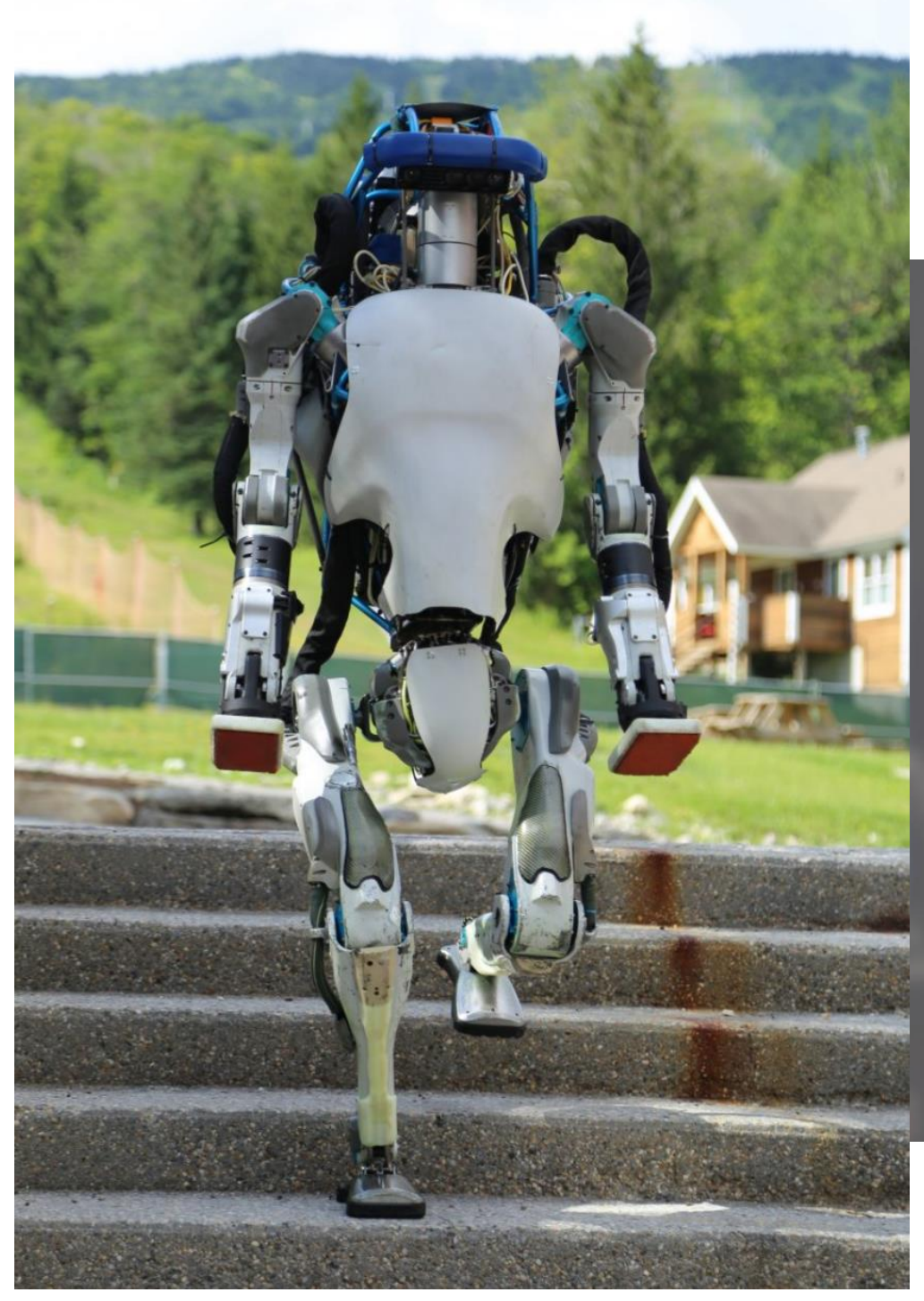




\section{Emerging Technology}

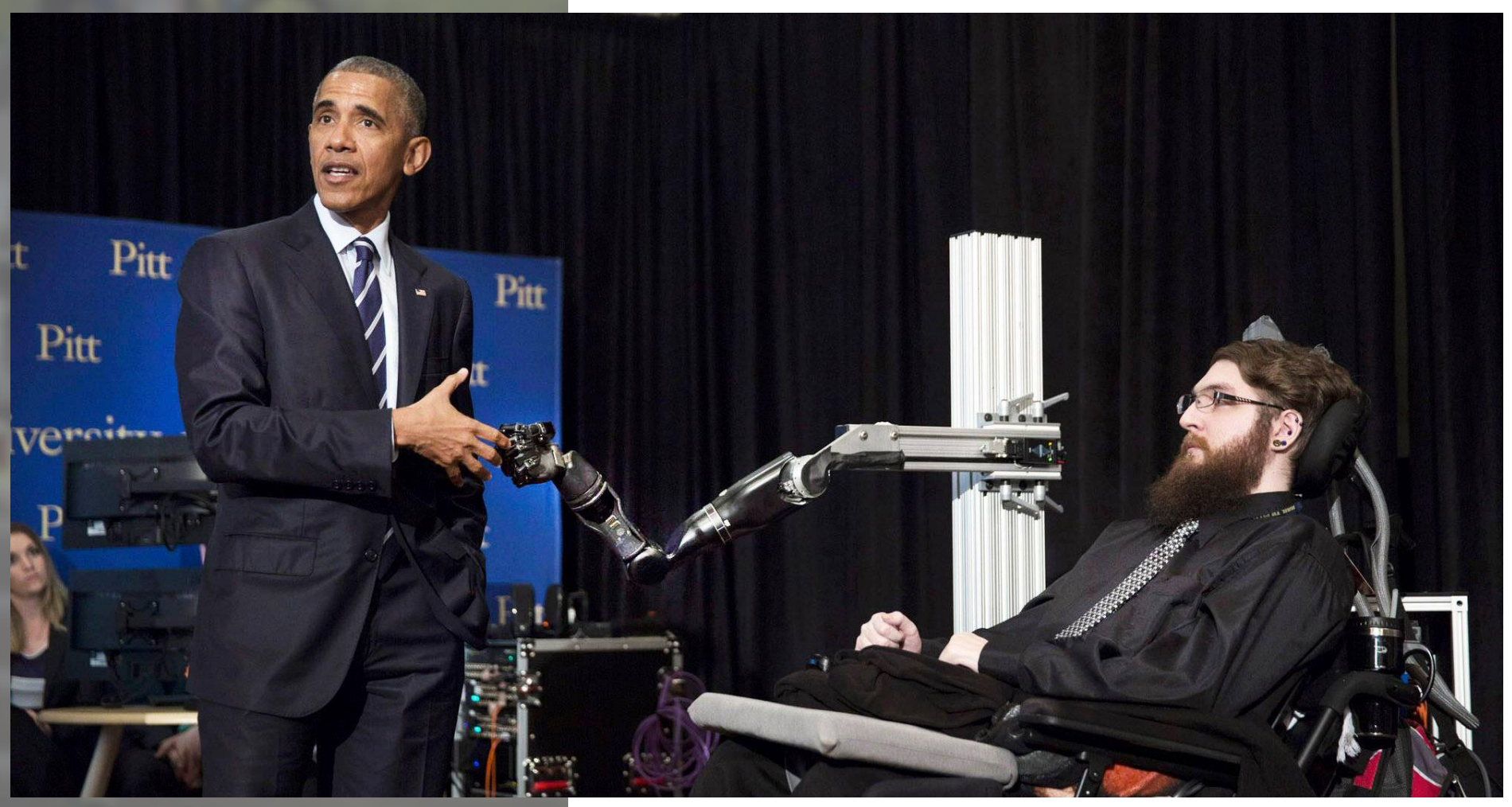




\section{What is Al?}

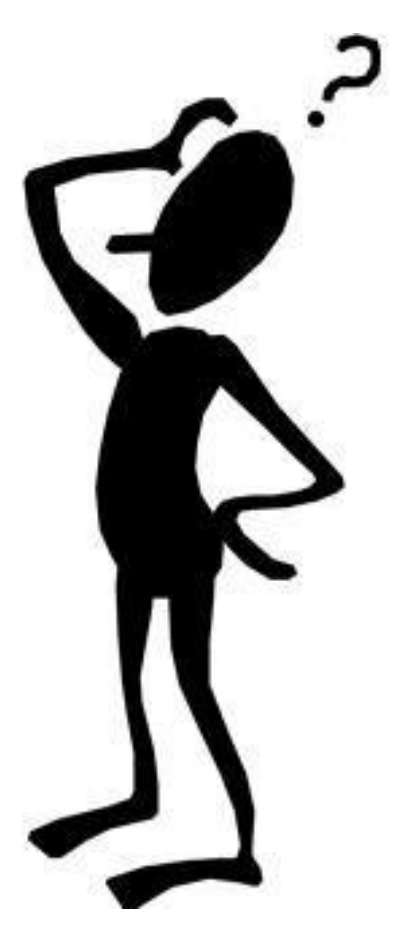




\section{What is Al?}

- Think and act like humans

- Think and act rationally

- Sometimes mutually exclusive. (;)

- Al Problem Space Categories

- Knowledge Representation

- Perception

- Logical Reasoning

- Planning and Navigation

- Prediction 


\section{Al Conceptualization (1 of 3)}

Embodied

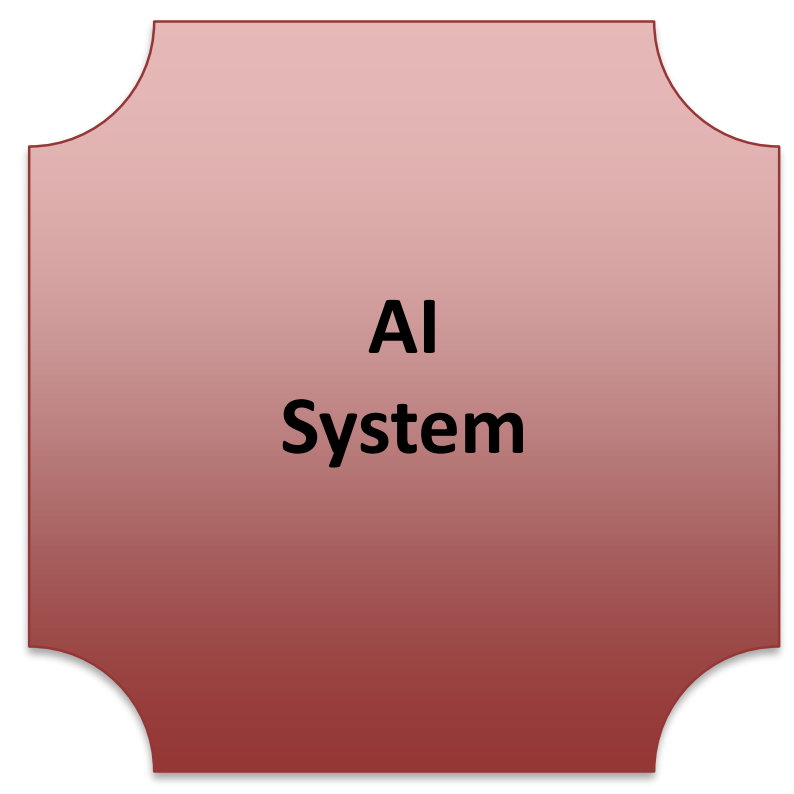

Embedded

Application/

System

Al

Technology 


\section{Al Conceptualization (2 of 3)}

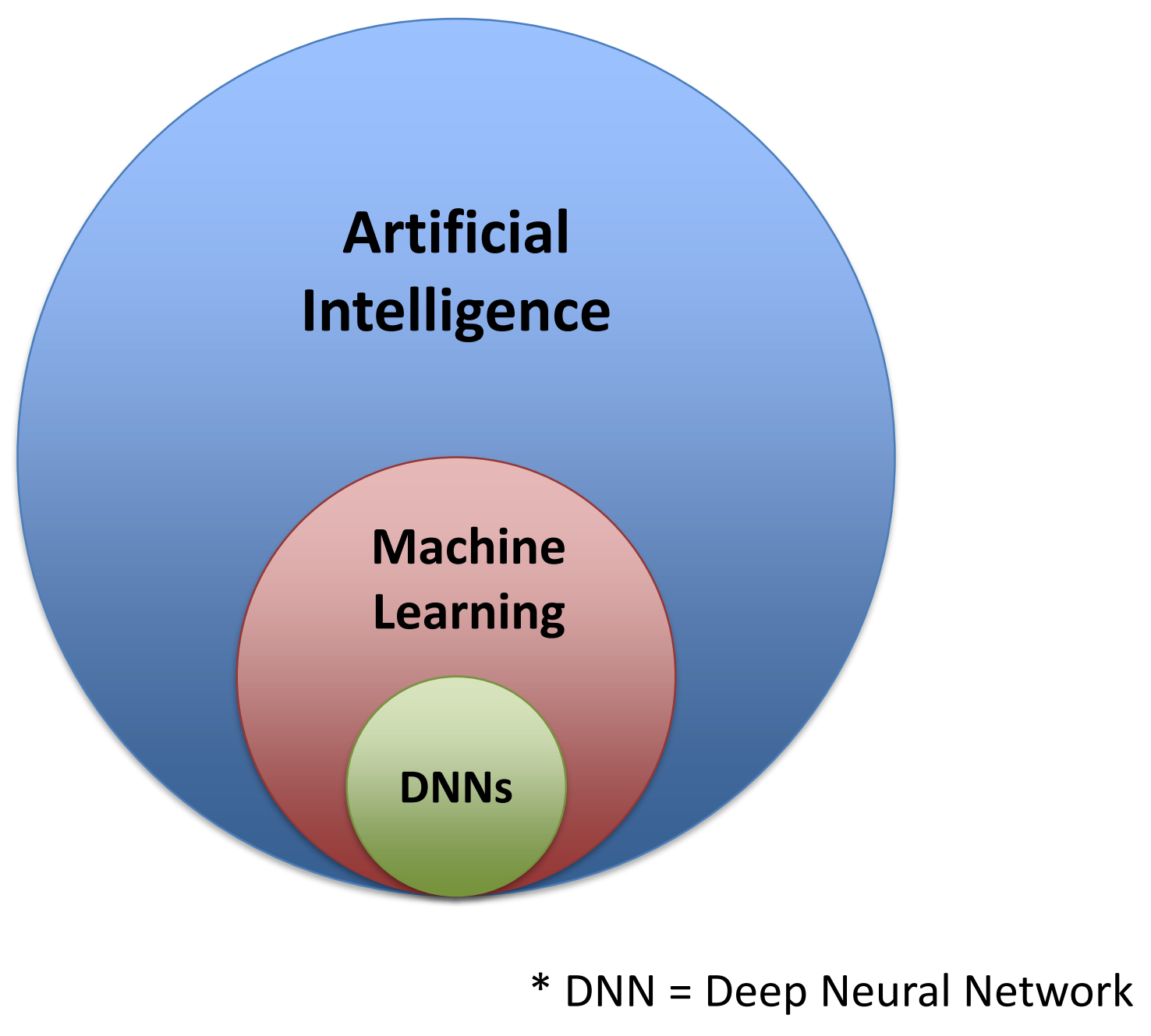




\section{Al Conceptualization (3 of 3)}

\begin{tabular}{|c|c|}
\hline Narrow Al & General Al \\
\hline - Application specific/ task limited & $\begin{array}{l}\text { O Perform general (human) intelligent } \\
\text { action }\end{array}$ \\
\hline $\begin{array}{l}\text { O Fixed domain models provided by } \\
\text { programmers }\end{array}$ & $\begin{array}{l}\text { Self-learns and reasons with its } \\
\text { operating environment }\end{array}$ \\
\hline $\begin{array}{l}\text { O Learns from thousands of labeled } \\
\text { examples }\end{array}$ & $\begin{array}{l}\text { Learns from few examples and/or from } \\
\text { unstructured data }\end{array}$ \\
\hline O Reflexive tasks with no understanding & O Full range of human cognitive abilities \\
\hline $\begin{array}{l}\text { Knowledge does not transfer to other } \\
\text { domains or tasks }\end{array}$ & $\begin{array}{l}\text { L Leverages knowledge transfer to new } \\
\text { domains and tasks }\end{array}$ \\
\hline o Today's Al & O Future Al? \\
\hline
\end{tabular}




\section{New Wave of Al}

- Availability of Big Data

- Improved Machine Learning (ML) Algorithms

- More Powerful Computing

- Mobile Connectivity

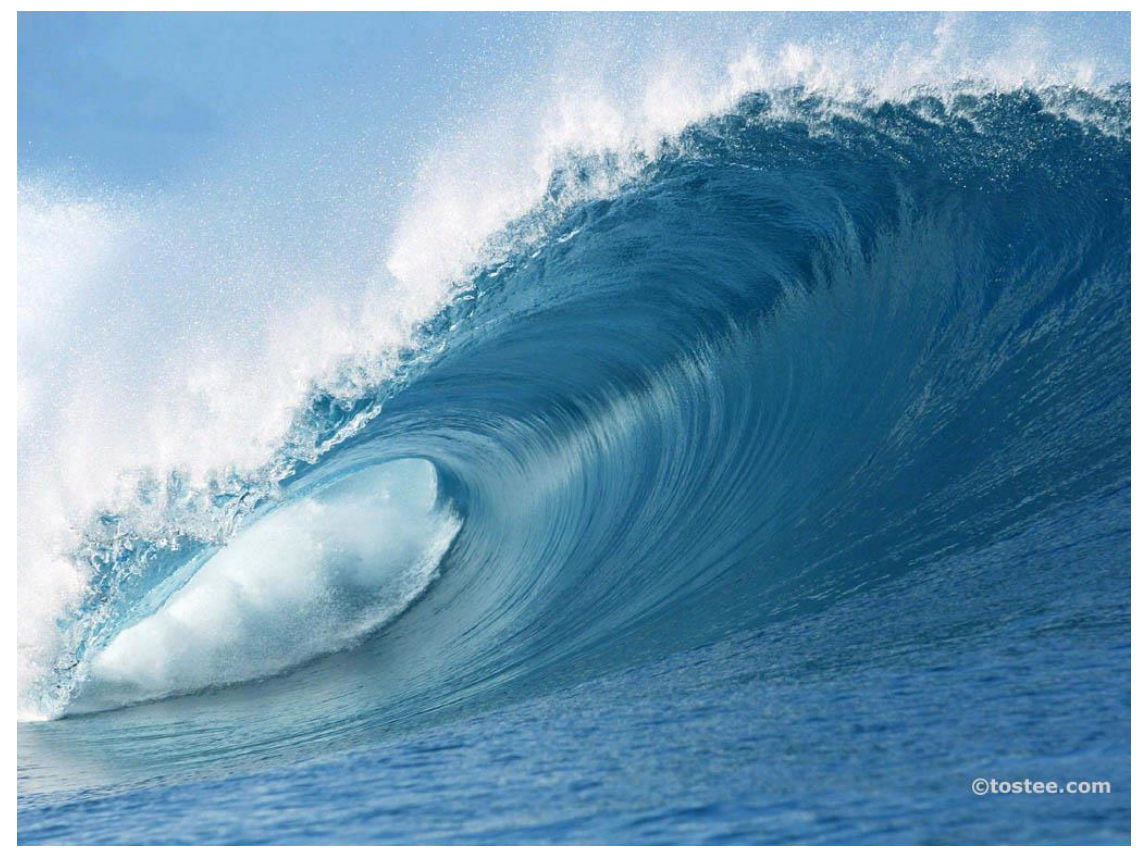




\section{What can ML/Al do?}

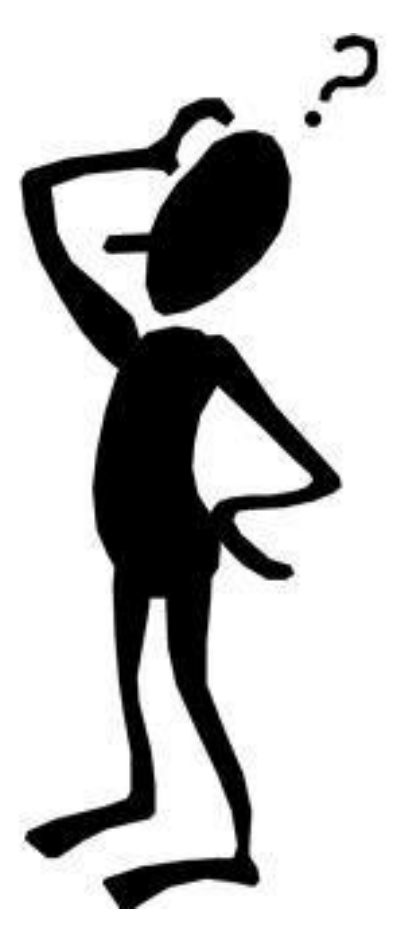




\section{ML/Al for ...}

- Classification

- Function Approximation

- Prediction

- Control

- Simulation

- Anomaly Detection 


\section{ML/Al for Manufacturing (1 of 2)}

- Advanced Data Analytics*

- Predictive Maintenance

- Yield, Throughput, Resource Efficiency

- End-to-End Systems Optimization

- Including Integrated Supply Chain 


\section{ML/Al for Manufacturing (2 of 2)}

- Human Assistive Technologies

- Automated Inspection and Quality Control

- Collaborative Robots

- Virtual and Augmented Reality

- Training and enhanced context awareness

- Workforce Knowledge Base

- ... 


\section{Machine Learning Workflow}

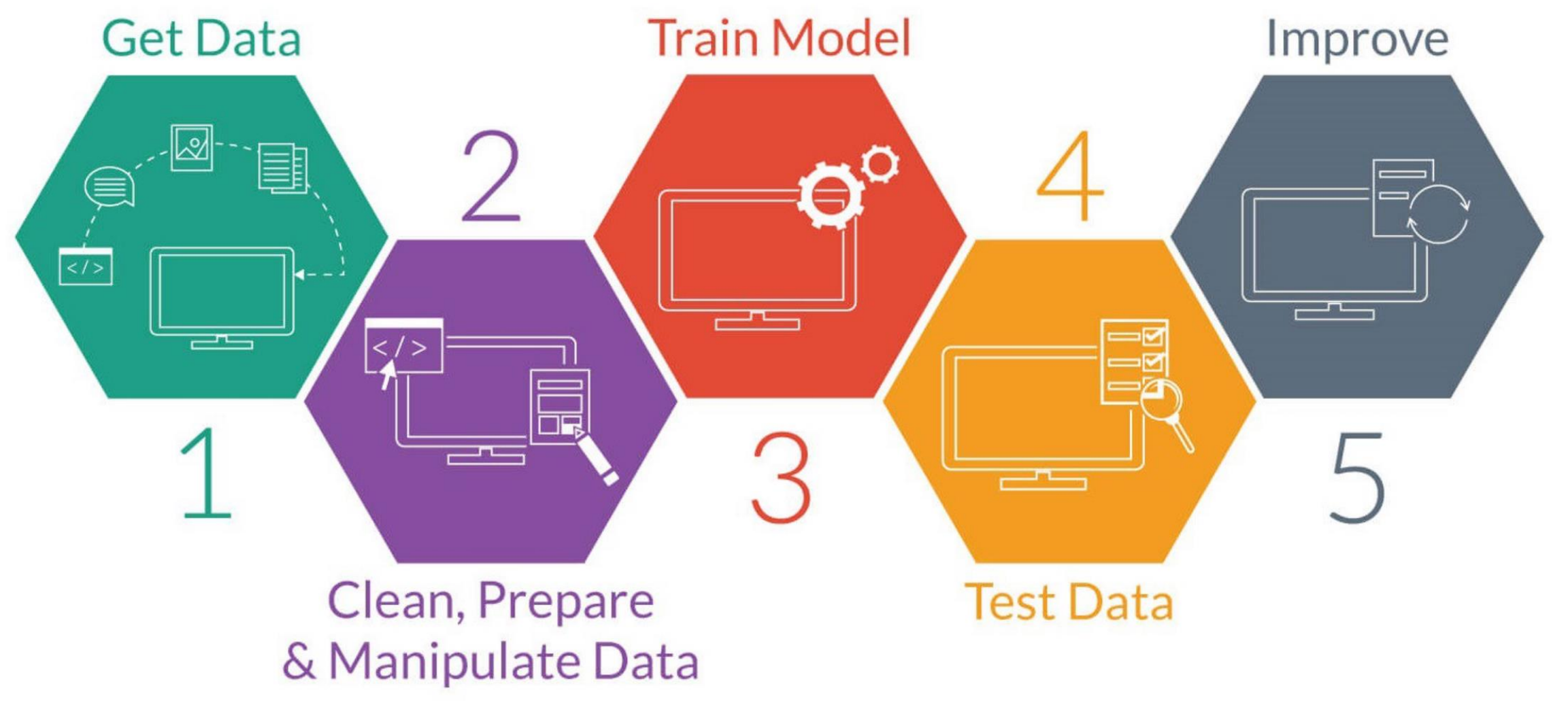




\section{ML/Al Challenges}

- Probabilistic

$\circ$ With inherent error rates and uncertainty

- Data Driven

- With vulnerability to learning unwanted patterns of bias

- Extreme Dimensionality

o Making decisions opaque (little explainability) 


\section{Summary: Today's ML/AI ...}

- Powerful Tool

- Great Impact on Manufacturing

- Challenges and Limitations

- Life Cycle Costs 


\title{
Questions?
}

Michael Garris

\author{
(mgarris@nist.gov)
}

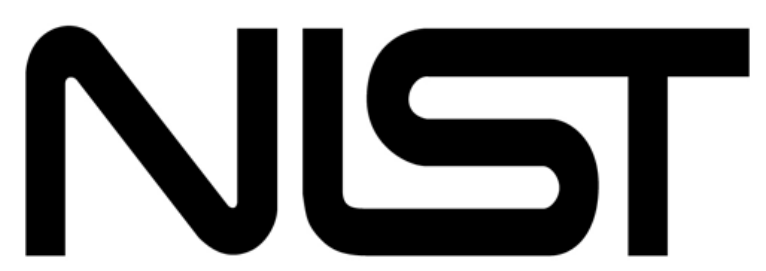

